\title{
The Involvement of Sigma Receptor Modulation in the Antidepressant Effects of Ketamine and the Neurotoxic Actions of Methamphetamine
}

\author{
Matthew J. Robson \\ West Virginia University
}

Follow this and additional works at: https://researchrepository.wvu.edu/etd

\section{Recommended Citation}

Robson, Matthew J., "The Involvement of Sigma Receptor Modulation in the Antidepressant Effects of Ketamine and the Neurotoxic Actions of Methamphetamine" (2013). Graduate Theses, Dissertations, and Problem Reports. 3661.

https://researchrepository.wvu.edu/etd/3661

This Dissertation is protected by copyright and/or related rights. It has been brought to you by the The Research Repository @ WVU with permission from the rights-holder(s). You are free to use this Dissertation in any way that is permitted by the copyright and related rights legislation that applies to your use. For other uses you must obtain permission from the rights-holder(s) directly, unless additional rights are indicated by a Creative Commons license in the record and/ or on the work itself. This Dissertation has been accepted for inclusion in WVU Graduate Theses, Dissertations, and Problem Reports collection by an authorized administrator of The Research Repository @ WVU.

For more information, please contact researchrepository@mail.wvu.edu. 


\title{
The Involvement of Sigma Receptor Modulation in the Antidepressant Effects of Ketamine and the Neurotoxic Actions of Methamphetamine
}

\author{
Matthew J. Robson \\ A dissertation submitted to the School of Pharmacy at \\ West Virginia University in partial fulfillment of the requirements for the degree of
}

Doctor of Philosophy in Pharmaceutical and Pharmacological Sciences

Rae R. Matsumoto, Ph.D., Chair

Jason D. Huber, Ph.D.

James O'Donnell, Ph.D.

Carl R. Sullivan, M.D.

William Petros, Pharm.D.

Department of Basic Pharmaceutical Sciences

Morgantown, West Virginia

2013

Keywords: Sigma receptor, Ketamine, Methamphetamine, Depression, Neurotoxicity 


\begin{abstract}
$\underline{\text { ABSTRACT }}$
The Involvement of Sigma Receptor Modulation in the Antidepressant-Like Effects of Ketamine
\end{abstract}

Matthew J. Robson

Depression is estimated to affect at least $30 \%$ of the world's population at some point during their lives. Currently marketed antidepressants require a period of at least 2 to 3 weeks to display any antidepressant effects in clinical populations and rates of clinical resistance to the antidepressant effects of these drugs are high. Ketamine is an N-methyl-D-aspartate (NMDA) antagonist and dissociative anesthetic that has been shown to display rapid acting and prolonged antidepressant activity in smallscale human clinical trials. Ketamine also binds to sigma receptors, which are believed to be protein targets for a potential new class of antidepressant medications. The purpose of this study was to determine the involvement of sigma receptors in the antidepressant-like actions of ketamine. Competition binding assays were performed to assess the affinity of ketamine for sigma-1 and sigma-2 receptors. The antidepressant-like effects of ketamine were assessed in vitro using a neurite outgrowth model and PC12 cells, and in vivo using the forced swim test. The sigma receptor antagonists, 4methoxy-3-(2-phenylethoxy)- $N, N$-dipropylbenzeneethanamine hydrochloride (NE-100) and $N$-[2-(3,4dichlorophenyl)ethyl]- $N$-methyl-2-(dimethylamino)ethylamine dihydrobromide (BD1047), were evaluated in conjunction with ketamine in these assays to determine the involvement of sigma receptors in the antidepressant-like effects of ketamine. Ketamine bound to both sigma-1 and sigma-2 receptors with $\mu \mathrm{M}$ affinities. Additionally, ketamine potentiated nerve growth factor (NGF)-induced neurite outgrowth in PC12 cells and this effect was attenuated in the presence of NE-100. Ketamine also displayed antidepressant-like effects in the forced swim test; however, these effects were not attenuated by pretreatment with NE-100 or BD1047. Taken together, these data suggest that sigma receptor-mediated neuronal remodeling may contribute to the antidepressant effects of ketamine. 


\section{DEDICATION}

This dissertation is dedicated to

The most supportive, loving parents imaginable

James E. and Katherine S. Robson

The memory of my late grandmother, Katherine Robson, who taught me life lessons I shall never forget...

And my loving wife and best friend

Catherine E. Robson 


\section{ACKNOWLEDGEMENTS}

I would like to first acknowledge the support and mentorship I received from, Dr. Rae R. Matsumoto. Without her guidance, encouragement, support and mentorship none of what is included in the current dissertation would have been possible. She has been a constant source of inspiration and motivation to pursue many of the opportunities presented to me within the last four years. Her guidance has allowed me to expand not only as a scientist, but as an individual as well. My sincerest gratitude goes out to her for all of the opportunities and memories during my time at West Virginia University.

Secondly, I wish to thank the remainder of my committee members, Jason Huber, Ph.D., James O'Donnell, Ph.D., Carl Sullivan, M.D. and William Petros, Pharm. D. for valuable insight into many of the projects contained within this dissertation. I would especially like to thank Jason Huber, Ph.D. for the collaborative experiences with members of his lab on several projects in the current dissertation. Without these collaborations, much of this work would not have been possible.

I would like to also thank our medicinal chemist collaborators, Christopher McCurdy, Ph.D. at the University of Mississippi and Andrew Coop, Ph.D. at the University of Maryland for synthesizing many of the compounds utilized in these studies.

I would also like to thank members of the Matsumoto lab, both past and present. Michael Seminerio, Ph.D., Meenal Elliott, Ph.D., Jason Healy, Yantong Xu, Ph.D., Jamaluddin Shaikh, Ph.D., James Fishback, Ph.D., Nidhi Kaushal, Ph.D., Bahar Noorbakhsh, and Christina Byrne-Hoffman. I would also like to thank members of the Huber lab, including Ryan Turner, Zachary Naser and Aric Logsdon.

Lastly, I would like to say thank you to my parents, James and Katherine, my sister, Melissa, and my wife, Catherine, for all the years of love, support and guidance that has helped me achieve many of my goals in life thus far. 


\section{$\underline{\text { TABLE OF CONTENTS }}$}

ABSTRACT

LIST OF FIGURES

LIST OF TABLES

ABBREVIATIONS

CHAPTER 1. Introduction: Ketamine, Depression and Sigma Receptors ........................................... 1

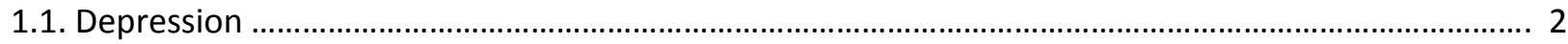

1.2. Currently Utilized Treatments for Depression ............................................................................. 2

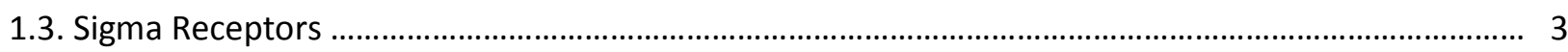

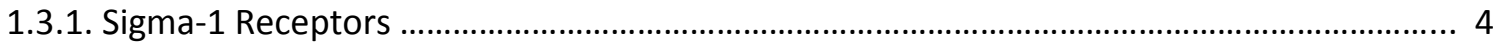

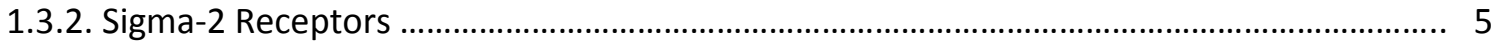

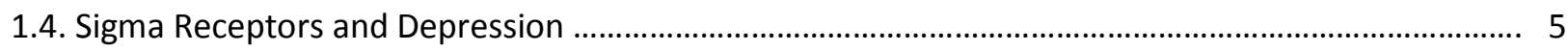

1.5. Sigma Receptors and Glutamatergic Signaling ...................................................................

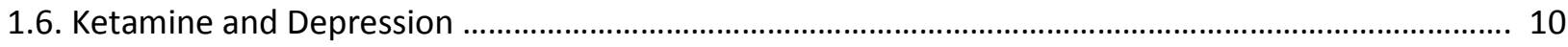

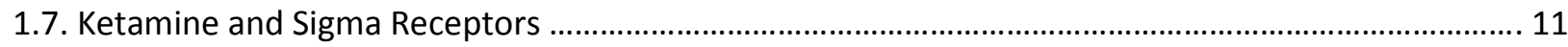

CHAPTER 2. Evaluation of Sigma Receptors in the Antidepressant-Like Effects of Ketamine

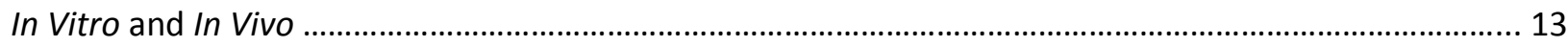

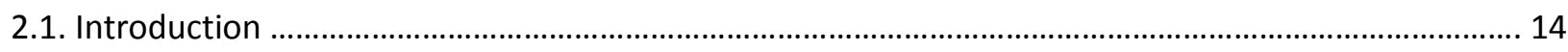

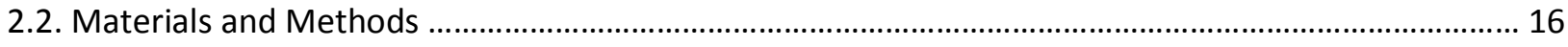

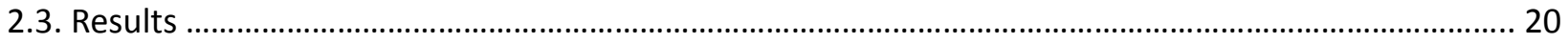

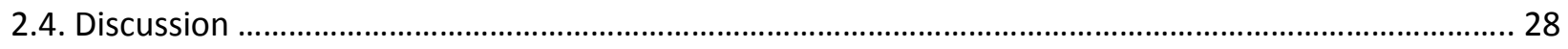

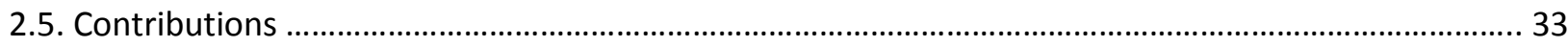


CHAPTER 3. Methamphetamine Abuse and Neurotoxicity.............................................................. 35

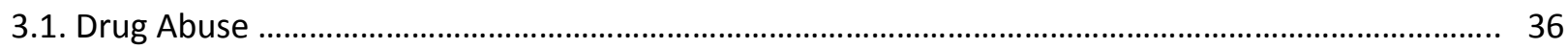

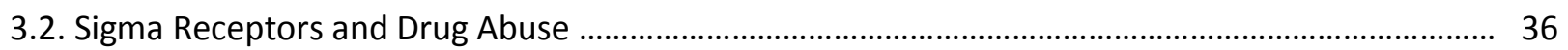

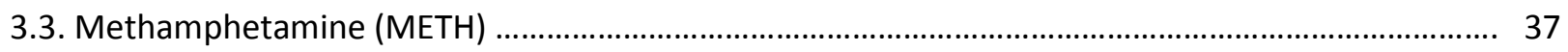

3.4. METH-Induced Neurotoxicity: Potential Mechanisms .................................................................. 39

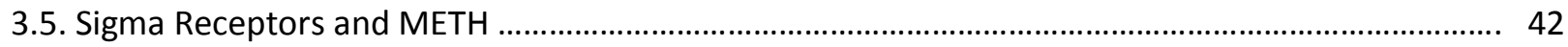

3.6. Sigma Receptor Ligands as Potential Treatments for the Negative Side Effects of METH............. 44

CHAPTER 4. Sigma Receptor Antagonist Attenuation of METH-Induced Neurotoxicity is Correlated to

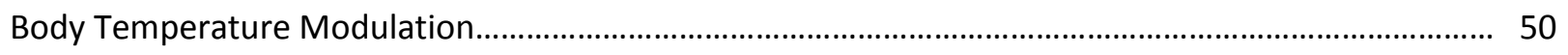

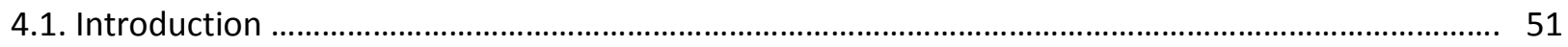

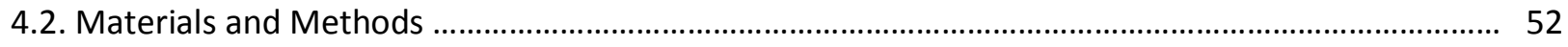

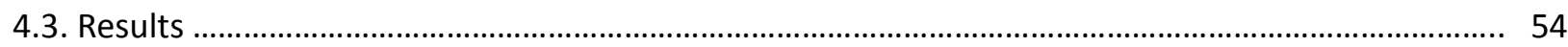

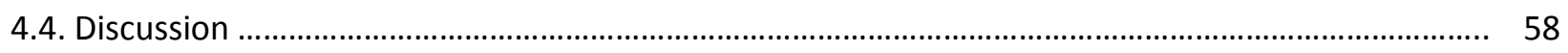

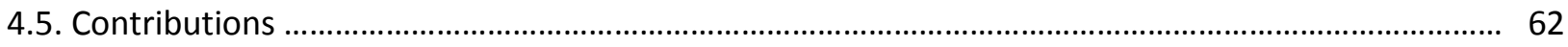

CHAPTER 5. AC927 Pretreatment Mitigates METH-Induced Changes in Striatal Gene Expression ....... 63

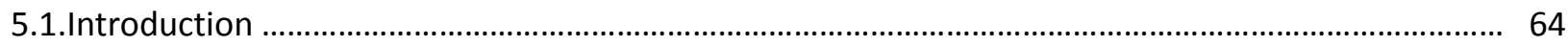

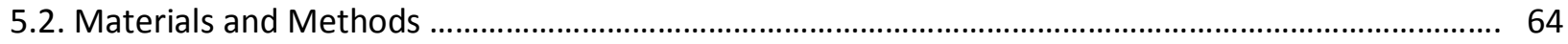

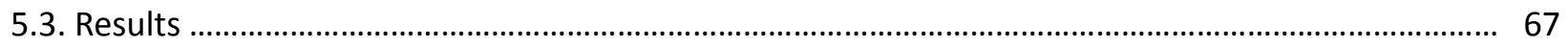

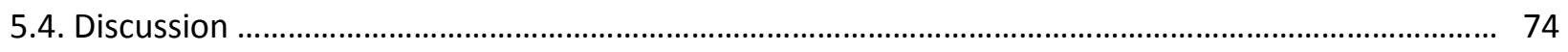


CHAPTER 6. METH Causes PERK-Mediated Endoplasmic Reticulum Stress in NG108-15 Cells Through a Mechanism Independent of Sigma Receptors.................................................................................

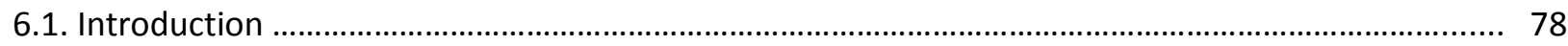

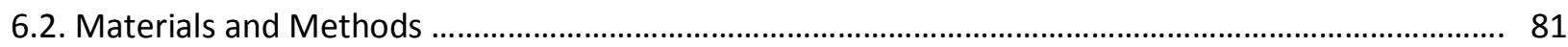

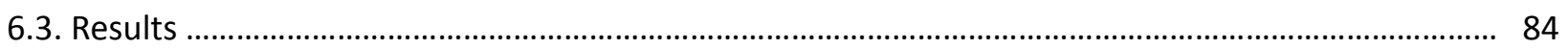

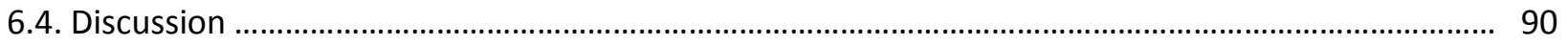

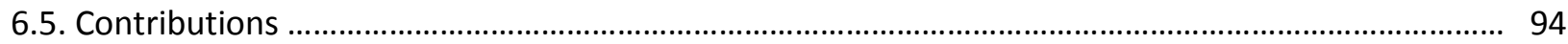

CHAPTER 7. SN79, A Sigma Receptor Ligand, Blocks METH-Induced Microglial Activation and Cytokine

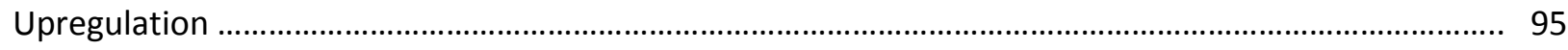

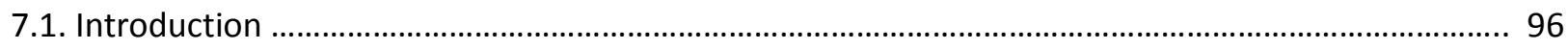

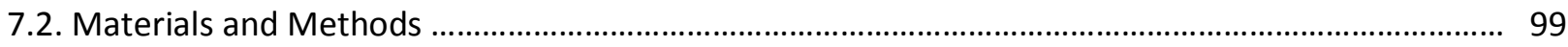

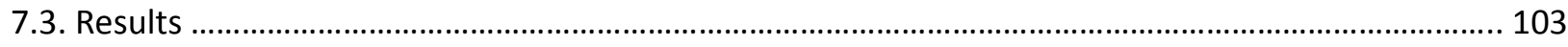

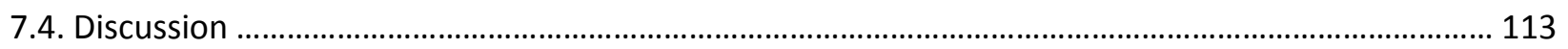

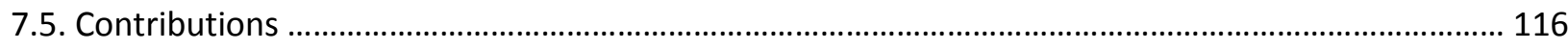

CHAPTER 8. SN79, A Sigma Receptor Ligand, Attenuates METH-Induced Astrogliosis Through a Blockade

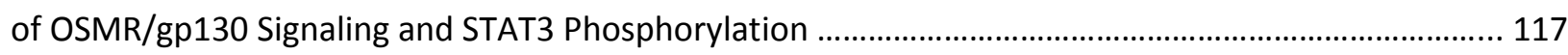

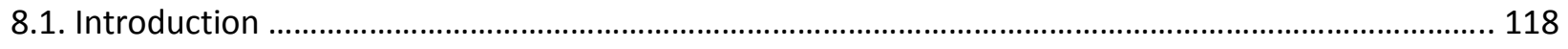

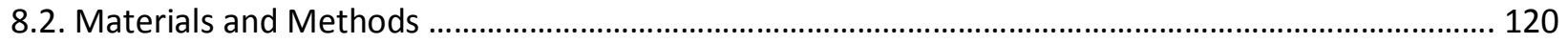

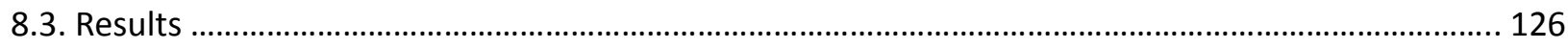

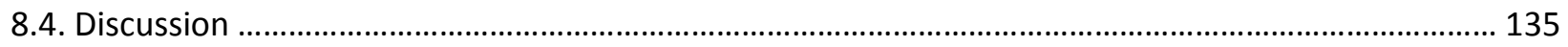


CHAPTER 9. Summary and Conclusions

REFERENCES 


\section{LIST OF FIGURES}

Figure 1.1. Sigma receptor modulation of cellular signaling relevant to depression

Figure 2.1. Effects of imipramine, ketamine and NE-100 on NGF-induced neurite outgrowth

Figure 2.2. Effects of NE-100 on NGF-induced neurite outgrowth potentiated by imipramine and

ketamine 24

Figure 2.3. Antidepressant-like effects of imipramine, ketamine, NE-100 and BD1047 in the forced swim

test 26

Figure 2.4. Antidepressant-like effects of BD1047 + ketamine and NE-100 + ketamine in the forced swim test . . 27

Figure 2.5. Time course of the antidepressant-like effects of ketamine in the forced swim test 28

Figure 3.1. Proposed mechanisms of METH-induced dopaminergic neurotoxicity 41

Figure 4.1. Body temperature measurements of experiments using AC927 and AZ66 55

Figure 4.2. Striatal dopamine measurements from AC927 and AZ66 hyperthermia experiments 56

Figure 4.3. Correlation of the attenuation of METH-induced hyperthermia and METH-induced reductions in striatal dopamine by AC927 and AZ66 58

Figure 6.1. PERK-mediated ER stress pathway 79

Figure 6.2. METH treatment causes an upregulation of genes involved in PERK ER stress in NG108-15

cells 85

Figure 6.3. METH treatment results in a dose dependent increase in CHOP expression 87

Figure 6.4. SN79 treatment potentiates METH-induced atf4 mRNA expression 89

Figure 6.5. Treatment with SN79 does not alter METH-induced CHOP expression 90

Figure 7.1. SN79 mitigates METH-induced hyperthermia 104

Figure 7.2. METH time dependently increases cd68 mRNA expression 105

Figure 7.3. SN79 blocks METH-induced increases in striatal CD68 mRNA and protein expression ....... 106 
Figure 7.4. SN79 blocks METH-induced increases in striatal IBA-1 expression

Figure 7.5. METH does not alter levels of M2 microglia in the striatum 109

Figure 7.6. METH treatment increases mRNA expression of IL-6-type cytokines in the striatum 111

Figure 7.7. SN79 pretreatment blocks METH-induced increases in IL-6-type cytokine mRNA expression in the striatum 112

Figure 8.1. METH treatment time dependently increases striatal gfap mRNA expression 126

Figure 8.2. SN79 treatment mitigates METH-induced increases in striatal gfap mRNA expression .....

Figure 8.3.A. Qualitative images of astrocyte activation in response to METH and a blockade by SN79

treatment 128

Figure 8.3.B. Quantification of astrocyte activation in response to METH and a blockade by SN79

treatment 128

Figure 8.4. SN79 treatment blocks METH-induced increases in striatal osmr mRNA expression 129

Figure 8.5. METH treatment increases the expression of OSMR in astrocytes 130

Figure 8.6. SN79 blocks METH-induced increases in the phosphorylation of STAT3 132

Figure 8.7. SN79 mitigates METH-induced striatal neuronal degeneration 137

Figure 8.8. Proposed OSM signaling pathway relevant to the activation of astrocytes by METH 136 


\section{LIST OF TABLES}

Table 1.1. Affinities of currently marketed antidepressants for sigma-1 and sigma-2 receptors .......... 6

Table 2.1. Affinities of utilized compounds for sigma-1 and sigma-2 receptors .................................... 21

Table 3.1. Chemical structures of sigma receptor ligands known to mitigate the neurotoxic effects of

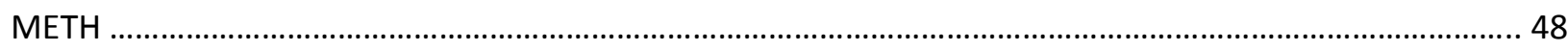

Table 5.1. List of genes obtained through ANOVA and fold change greater than 2 analysis................... 67

Table 5.2. List of genes obtained through ANOVA and FDR correction analysis.................................... 69 


\section{ABBREVIATIONS}

AC927, 1-(2-phenylethyl)piperidine; AMPA, $\alpha$-amino-3-(3-hydroxy-5-methyl-isoxazol-4-yl)propanoic acid; ANOVA, analysis of variance; ATF3, activating transcription factor 3; ATF6, activating transcription factor 6; ATP, adenosine triphosphate; AZ66, 3-(4-(4-cyclohexylpiperzin-1-yl)pentyl)-6-fluorobenzo[d]thiazol2(3H)-one; BD1047, N-[2-(3,4-dichlorophenyl)ethyl]- $N$-methyl-2-(dimethylamino)ethylamine dihydrobromide; BD1063, 1-[2-(3,4-dichlorophenyl)ethyl]-4-methylpiperazine dihydrochloride; BDNF, brain derived neurtrophic factor; $\mathrm{BiP}$, glucose relates protein; $\mathrm{Ca}^{2+}$, calcium; $\mathrm{CD68}$, cluster of differentiation 68; CHOP, C/EBP homologous protein; CNS, central nervous system; D1R, dopamine receptor D1; DA, dopamine; DAQ, dopamine quinone; DAT, dopamine transporter; DTG, 1,3-di-(2tolyl)guanidine; eEF2, eukaryotic elongation factor 2; eIF2 $\alpha$, eukaryotic initiation factor-2 $\alpha$; ER, endoplasmic reticulum; FST, forced swim test; GADD34, protein phosphatase 1 regulatory subunit 15A; GFAP, glial fibrillary acidic protein; gp130, glycoprotein 130; HRP, horseradish peroxidase; IBA-1, ionized calcium-binding adapter molecule 1; IL-6, interleukin-6; IP ${ }_{3}$, inositol triphosphate; IRE-1 $\alpha$, inositolrequiring enzyme $1 \alpha$; Lif, leukemia inhibitory factor; LPS, lipopolysaccharide; MAOI, monoamine oxidase inhibitor; MAM, mitochondrial-associated endoplasmic reticulum membrane; MDMA, 3,4methylenedioxymethamphetamine; METH, methamphetamine; MS-377, (R)-(+)-1-(4-chlorophenyl)-3-[4(2-methoxyethyl)piperazin-1-yl]methyl-2-pyrrolidinone L-tartrate; NBQX, 2,3-dioxo-6-nitro-1,2,3,4tetrahydrobenzo[f]quinoxaline-7-sulfonamide; NE-100, 4-methoxy-3-(2-phenylethoxy)- $\mathrm{N}, \mathrm{N}$-dipropylbenzeneethanamine hydrochloride; NG108-15, neuroblastoma-glioma, hybridoma cells; NGF, nerve growth factor; NMDA, N-methyl-D-aspartate; NRI, norepinephrine reuptake inhibitor; OB, olfactory bulbectomy; OSM, oncostatin $\mathrm{m}$; OSMR, oncostatin $\mathrm{m}$ receptor; PBS, phosphate buffered saline; PC12, rat pheochromocytoma cells; PCP, phencyclidine; PERK, PKR-like endoplasmic reticulum kinase; RNS, reactive nitrogen species; ROS, reactive oxygen species; SA4503, 1-(3,4dimethoxyphenethyl)-4-(3-phenylpropyl)piperazine dihydrochloride; SDS, sodium dodecyl sulfate; SERT, serotonin transporter; SK channel, $\mathrm{Ca}^{2+}$-activated K ${ }^{+}$channels; SN79, 6-acetyl-3-(4-(4-(4fluorophenyl)piperazin-1-yl)butyl)benzo[d]oxazol-2(3H)-one; SNRI, serotonin-norepinephrine reuptake inhibitor; SOCS3, suppressor of cytokine signaling 3; SSRI, Selective serotonin reuptake inhibitor; STAT3, signal transducer and activator of transcription 3; TST, tail suspension test; UMB23, 1-(3phenylpropyl)piperidine oxalate; VMAT2, vesicular monoamine transporter-2 


\section{Chapter 1}

Introduction: Ketamine, Depression and Sigma Receptors 


\subsection{Depression}

Major depressive disorder is characterized by periods of loss of interest, general low mood and low self esteem that can lead to negative lifestyle changes and suicides or suicidal ideation . Depression is currently one of the most common health problems worldwide with an estimated 340 million people suffering from it at some point during their lives (Kulkarni and Dhir, 2009a). Although treatments have been developed, it has been estimate that up to $75 \%$ of people suffering from depression remain either untreated or treated with medications lacking sufficient efficacy (Kessler et al., 2007). Current pharmacologic treatments typically take weeks to exert any effect and are ineffective for up to $50 \%$ of patients (Berton and Nestler, 2006; Fava and Davidson, 1996; Kulkarni and Dhir, 2009a; Nierenberg, 2001). Therefore, there is a clear need for better antidepressant treatments, particularly ones that are fast-acting and able to treat those patients who fail to respond to currently available therapies.

\subsection{Currently Utilized Treatments for Depression}

Treatments for depression arose in a serendipitous fashion in the 1950 s as medications such as iproniazid and imipramine were found to display antidepressant effects in humans (Ball and Kiloh, 1959; Deverteuil and Lehmann, 1958). These two medications, a monoamine oxidase inhibitor (MAOI) and a tricyclic antidepressant, respectively, were found to alter monoamine neurotransmission thereby linking monoamine neurotransmission to depression (Blier and Bouchard, 1994; Chaput et al., 1991; Slattery et al., 2004). Subsequently, pharmacologic agents targeting serotonergic and noradrenergic signaling were produced namely, selective sertotonin reuptake inhibitors (SSRI's) and serotonin-norepinephrine reuptake inhibitors (SNRI's), as well as norepinephrine reuptake inhibitors (NRI's) (Murrough and Charney, 2012). Although these compounds have fewer deleterious side effects than their MAOI and tricyclic predecessors, these compounds suffer from poor and delayed clinical efficacy and high rates of non-response in clinical populations (Murrough and Charney, 2012). Current research therefore centers 
on novel targets such as neurotrophic factors, the hypothalamic-pituitary axis, glutamatergic systems and novel receptors and proteins believed to be involved in mood disorders (Fishback et al., 2010b). One of these potential new targets for the production of pharmaceutical agents aimed at treating depression are sigma receptors.

\subsection{Sigma Receptors}

Sigma receptors were initially characterized as a sub-class of opioid receptors more than thirty years ago when they were discovered by Martin et al. (Martin et al., 1976). Sigma receptors were characterized through the interactions of (+)-benzomorphans with these proteins and they initially received their names from the "S" of (+)-SKF-10,047 (Matsumoto et al., 2007). The ability of (+)-isomers of benzomorphans to interact with sigma receptors as opposed to (-)-isomer benzomorphans which interact preferentially with opioid receptors led to the belief that sigma receptors were a distinct subset of opioid receptors, much like the g-protein coupled $\mu, \delta$, and $\kappa$ subtypes of opioid receptors (Iwamoto, 1981; Matsumoto et al., 2007; Vaupel, 1983; Young and Khazan, 1984). This was ultimately found to not be true; however this confusion persisted for many years.

In later years, due to the ability of (+)-SKF-10,047 to interact with the PCP binding sites of NMDA receptors, it was believed that sigma receptors may actually be the PCP binding site of NMDA receptors (Mendelsohn et al., 1985; Quirion et al., 1981; Sircar et al., 1986). Eventually, further binding studies clarified that sigma receptors were distinct protein entities and not the PCP binding site of NMDA receptors (Su, 1982; Tam and Cook, 1984), however this led to a great deal of confusion surrounding sigma receptors, some of which remains today . The advent of truly selective ligands for sigma receptors has allowed for further clarification of the actions of these proteins and the molecular cloning of one of the subtypes has paved the way for many of the functional characterizations of sigma-1 receptors that are known today. 


\subsubsection{Sigma-1 Receptors}

Sigma-1 receptors are 223 amino acid proteins that consist of a two-transmembrane structure and have a molecular weight of approximately 25 kDa (Aydar et al., 2002; Hellewell and Bowen, 1990; Kahoun and Ruoho, 1992; Kekuda et al., 1996; Mei and Pasternak, 2001; Pan et al., 1998). They are now known to be ligand-activated endoplasmic reticulum (ER) chaperone proteins that reside at the mitochondrial-associated ER membrane (Hayashi and Su, 2007b) and share nothing functionally in common with the three subclasses of opioid receptors. Upon stimulation through ligand activation, sigma-1 receptors are believed to alter intracellular signaling through protein-protein interactions and through translocation to different cellular compartments acting as chaperone proteins, modulating Gprotein coupled receptors, ion channels and signaling cascades such as protein kinases, $\mathrm{Ca}^{2+}$ levels and modulating $\mathrm{IP}_{3}$ signaling (Hayashi et al., 2000a; Hayashi and Su, 2003, 2007b; Kourrich et al., 2013; Kourrich et al., 2012; Moon et al., 2013; Navarro et al., 2010; Su and Hayashi, 2003; Takebayashi et al., 2002). Sigma-1 receptors are known to interact directly with a variety of protein partners including ankyrin B, heat shock protein 70, glucose-related protein (BiP) and certain potassium channels (Aydar et al., 2002; Hayashi and Su, 2001; Matsumoto et al., 2007). It is through the modulation of several of these protein-protein interactions and signaling events that sigma-1 receptors are believed to be involved in a variety of disease states including depression and drug abuse (Fishback et al., 2010b; Hayashi et al., 2011; Kourrich et al., 2013; Robson et al., 2012).

In addition to being present within the CNS, sigma-1 receptors are also present in high concentrations in the heart and spleen (Matsumoto et al., 2007; Novakova et al., 1995; Wolfe et al., 1997). The ability of sigma-1 receptors to interact with these various proteins while also being expressed in vastly different physiologic regions has made studying the function of sigma-1 receptors particularly perplexing. The location of sigma-1 receptors in various regions of the body and their inherent ability to modulate several of protein-protein interactions and signaling events are believed to make them 
potential therapeutic targets for a variety of diseases including depression and drug abuse (Ehmke, 2012; Fishback et al., 2010b; Robson et al., 2012).

\subsubsection{Sigma-2 Receptors}

Much less is currently known about sigma-2 receptors than sigma-1 receptors; however this subtype is smaller than sigma-1 receptors and is approximately $18-22 \mathrm{kDa}$ in size (Hellewell et al., 1994). Sigma-2 receptors are localized in lipid rafts at the plasma membrane, ER, mitochondria and Iysosomes (Gebreselassie and Bowen, 2004; Zeng et al., 2007). Although sufficient tools are currently lacking to study many of the mysteries surrounding sigma-2 receptors, they are highly upregulated in cancer cells and play a large role in cell death through the alteration of calcium signaling and sphingolipid products (Bowen, 2000; Vilner and Bowen, 2000; Wheeler et al., 2000). For this reason, they are currently believed to be viable targets for the production of novel therapeutics aimed at treating specific types of cancer (Spitzer et al., 2012; Zeng et al., 2012), and agents aimed at detecting tumors (Tu et al., 2010).

\subsection{Sigma Receptors and Depression}

The involvement of sigma receptors in the actions of antidepressant drugs was first suggested by observations that most marketed antidepressant drugs bind to these receptors (Table 1.1), raising the possibility that some of their therapeutic effects may be mediated via these proteins (Itzhak and Kassim, 1990; Narita et al., 1996a; Schmidt et al., 1989). Recently, it has been shown that sigma receptors are involved in the actions of fluvoxamine in the forced swim test (FST), whereas paroxetine's antidepressant-like effects in the FST are independent of sigma receptor activation (Sugimoto et al., 2012). Fluvoxamine is known to display significant affinity for sigma-1 receptors, whereas paroxetine 
does not have appreciable affinity for either subtype of sigma receptor (Fishback et al., 2010b; Narita et al., 1996b).

\begin{tabular}{|l|c|c|}
\hline Currently Marketed Antidepressant & Sigma-1 Receptor $\mathbf{K}_{\mathbf{i}}(\mathbf{n M})$ & Sigma-2 Receptor $\mathrm{K}_{\mathbf{i}}(\mathbf{n M})$ \\
\hline Fluvoxamine & 36 & 8439 \\
\hline Sertraline & 57 & 5297 \\
\hline Fluoxetine & 120 & 5480 \\
\hline Citalopram & 292 & 5410 \\
\hline Paroxetine & & 22,870 \\
\hline Imipramine & 1893 & 2107 \\
\hline Desipramine & 343 & 11,430 \\
\hline
\end{tabular}

Table 1.1. Affinities of currently marketed antidepressants for sigma-1 and sigma-2 receptors (Fishback et al., 2010b; Narita et al., 1996b).

Additionally, selective sigma receptor agonists exert antidepressant-like effects in the FST. Sigma receptor agonists such as di-o-tolylguaidine (DTG), igmesine, (+)-pentazocine, SA4503 and (1-(3phenylpropyl)piperdine oxalate (UMB23) have been shown to exert antidepressant-like effects in the FST, an effect that is able to be blocked by treatment with the sigma receptor antagonists $\mathrm{N}-[2-(3,4-$ dichlorophenyl)ethyl]-N-methyl-2-dimethylamino)ethylamine (BD1047) or N,N-dipropyl-2-[4-meth-oxy3-(2-phenylethoxy)phenyl]ethylamine (NE-100) (Matsuno et al., 1996; Wang et al., 2007b). Although many of these compounds have mixed affinity for sigma-1/sigma-2 receptors, sigma-1 receptors are beleived to be the most important subtype with regards to antidepressant activity, however the specific 
role of sigma-2 receptors in many of these effects has yet to be conclusively determined (Fishback et al., 2010b). Furthermore, studies specifically implicating sigma-1 receptors in these actions are those utilizing sigma-1 receptor knockout mice, revealing that these mice display a depressed-like phenotype as compared to wild-type controls (Sabino et al., 2009).

Further evidence that targeting sigma receptors may provide viable novel therapies aimed at treating depression are studies showing that sigma receptor ligands display antidepressant effects when utilized in human clinical populations. Igmesine hydrochloride is a sigma receptor ligand that has previously been shown in small scale trials to display significant antidepressant effects in patients suffering from depression with comparable efficacy to currently marketed antidepressants (Fishback et al., 2010b; Volz and Stoll, 2004). 


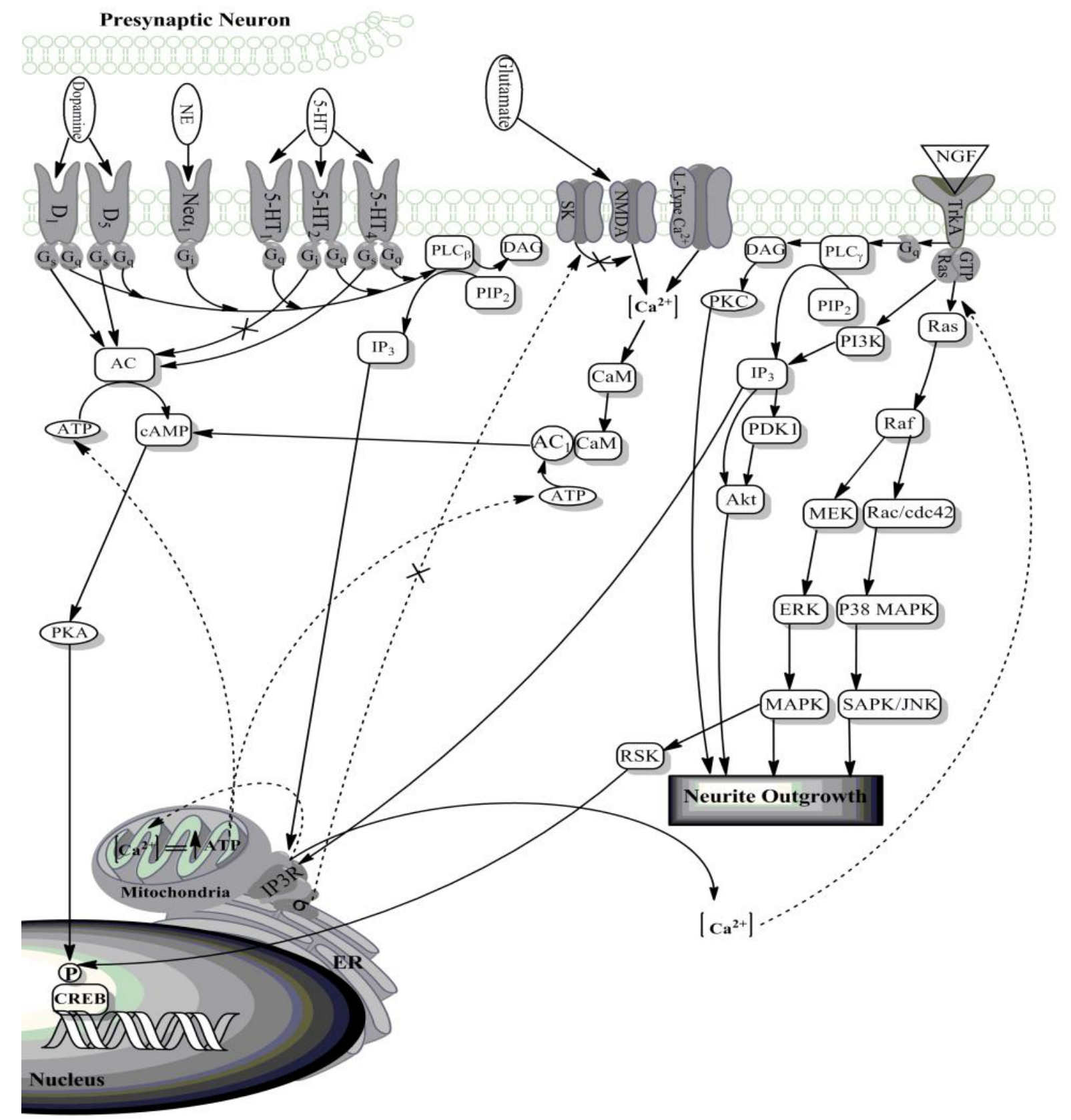

Figure 1.1. Sigma receptor modulation affects a variety of signaling pathways believed to be relevant to the etiology of depression. Sigma-1 receptors specifically, when activated, are able to translocate to distinct cellular compartments thereby interacting with and modulating several proteins, receptors and ion channels relevant to depression and the actions of currently available antidepressants. Sigma receptors modulate monoamine signaling, as well as glutamatergic signaling, in addition to pathways relevant to neurite outgrowth and synapse formation, all mechanisms hypothesized to be factors in the actions of antidepressant medications. 
The precise mechanisms by which sigma receptor ligands and sigma receptors themselves alter depressive phenotypes is currently unknown. It is known that sigma receptors alter many intercellular pathways related to the etiology of depression (Fishback et al., 2010b). The ways in which sigma receptors are known to modulate pathways relevant to depression are depicted in Figure 1.1.

In depth discussion of each and every proposed mechanism by which sigma receptors can modulate these pathways would be extensive and beyond the scope of the current dissertation. Additionally, it is the focus of several well written reviews that are currently published (Fishback et al., 2010b; Hayashi et al., 2011; Stahl, 2005; Su and Hayashi, 2003; Takebayashi et al., 2004; Tsai et al., 2009). The section below thus discusses the relationship between glutamatergic signaling and sigma receptors exclusively, as it relevant to work contained in the proceeding sections of this dissertation.

\subsection{Sigma Receptors and Glutamatergic Signaling}

Glutamatergic responses mediated through NMDA receptors are modulated by sigma receptor ligands. Specifically, sigma receptor activation results in an enhancement of NMDA neurotransmission (Bergeron et al., 1993; Bermack et al., 2002; Bermack and Debonnel, 2005; lyengar et al., 1990; Monnet et al., 1992a; Monnet et al., 1996). Sigma-1 receptor activation also potentiates glutamatergic responses in hippocampal neurons in vivo (Bergeron et al., 1995; Bergeron et al., 1993; Monnet et al., 1992b; Monnet et al., 1990). More recently, a potential mechanism by which this effect occurs was discovered. It is now known that sigma receptor antagonists such as (+)-pentazocine exert this effect by shunting small conductance $\mathrm{Ca}^{2+}$-activated $\mathrm{K}^{+}$channels (SK channels) (Martina et al., 2007). The ultimate effect of shunting SK channels is an enhancement of $\mathrm{Ca}^{2+}$ influx through NMDA receptors, thereby potentiating NMDA responses (Martina et al., 2007). This effect is illustrated in Figure 1.1.

Another way in which sigma receptor modulation may alter glutamatergic signaling that is relavant to the etiology of depression is through alterations in NMDA receptor expression. The sigma 
receptor agonist SA4503 has been shown to reverse a decrease in the expression of NR1 sububits of NMDA receptors in animals that have undergone an olfactory bulbectomy $(\mathrm{OB})$, a procedure that simulates many aspects of clinical depression in rodent models (Fishback et al., 2010b; Wang et al., 2007a). It should be noted that SA4503 displays significant antidepressant-like efffects in rodent models and the increase in NR1 subunits was blocked by administration of NE-100, a sigma receptor antagonist (Skuza and Rogoz, 2002; Wang et al., 2007a).

It is clear that sigma receptor modulation can alter glutamatergic neurotransmission; however, the exact implications of this are currently unknown and the ability of sigma receptors and their ligands to modulate glutamatergic signaling represents one potential mechanism by which sigma receptor ligands exert antidepressant-like effects.

\subsection{Ketamine and Depression}

As discussed above, there are several issues with currently utilized antidepressants. One of the largest problems is a significant delay in time (approximately 2-4 weeks) between treatment initiation and any antidepressant efficacy of currently utilized pharmacotherapies. This has led to significant research resources being aimed at finding antidepressant therapies with alternative mechanisms of action that may have rapid antidepressant effects while also targeting the approximately $30-50 \%$ of patients who fail to fail to respond to classical therapies.

Recent studies have begun to investigate the antidepressant-like properties of ketamine through a variety of approaches (aan het Rot et al., 2010; Berman et al., 2000; Diazgranados et al., 2010a; Diazgranados et al., 2010b; Kollmar et al., 2008; Li et al., 2010b; Machado-Vieira et al., 2009; Paul et al., 2009; Popik et al., 2008; Price et al., 2009; Zarate et al., 2006). Ketamine is an NMDA receptor antagonist and dissociative anesthetic, that has been shown to display rapid acting antidepressant activity in human clinical populations in several small cohort trials (aan het Rot et al., 2010; Berman et 
al., 2000; Diazgranados et al., 2010a; Diazgranados et al., 2010b; Zarate et al., 2006). These studies are especially significant because they focus on utilizing ketamine for patients with treatment resistant major depressive disorder who have clinically failed on currently available therapeutics (aan het Rot et al., 2010; Diazgranados et al., 2010b; Zarate et al., 2006). In treatment resistant patients, therapeutic effects were observed within hours after ketamine administration, with antidepressant effects of the treatment persisting for up to seven days (Zarate et al., 2006).

It is believed that modulation of glutamatergic signaling through NMDA and $\alpha$-amino-3-(3 hydroxy-5-methyl-isoxazol-4-yl)propanoic acid (AMPA) receptors is responsible for the antidepressant actions of ketamine (Maeng et al., 2008). It is hypothesized that increased AMPA signaling may underlie the antidepressant effects of ketamine, as treatment with the AMPA antagonist 2,3-dioxo-6-nitro1,2,3,4-tetrahydrobenzo[f]quinoxaline-7-sulfonamide (NBQX) has been shown to block the antidepressant-like actions of ketamine in the FST and the tail suspension test (TST) in rodents (Koike et al., 2011; Maeng et al., 2008). A more specific mechanism however has been proposed that focuses on a rapid increase in brain-derived neurotrophic factor (BDNF), mediated through eukaryotic elongation factor 2 (eEF2) kinase signaling through a modulation of NMDA receptors at rest by ketamine as a mechanism behind the antidepressant actions of the drug (Autry et al., 2011; Kavalali and Monteggia, 2012).

\subsection{Ketamine and Sigma Receptors}

In addition to binding NMDA receptors and modulating glutamatergic signaling, ketamine interacts with several other protein targets, including sigma receptors (Hustveit et al., 1995; Seeman et al., 2009). As discussed above, sigma receptor ligands have been shown to display antidepressant-like effects in animal models and antidepressant actions in human trials. The determination as to whether the antidepressant actions of ketamine are related to its interaction with sigma receptors, however, has 
yet to be made. The following chapter focuses on this interaction with the goal of determining if the antidepressant-like effects of ketamine involve sigma receptor modulation. The following three specific aims were proposed in order to evaluate the involvement of sigma receptors in the antidepressant-like effects of ketamine:

1. Identify the sigma receptor subtypes for which ketamine displays appreciable affinity

2. Determine the ability of ketamine to potentiate NGF-induced neurite outgrowth in vitro, and the role of sigma receptors in this effect.

3. Confirm that ketamine elicits antidepressant-like effects in the FST and determine the role of sigma receptors in these effects. 


\section{Chapter 2}

Evaluation of sigma receptors in the antidepressant-like effects of

ketamine in vitro and in vivo 


\subsection{Introduction}

Depression afflicts up to $20 \%$ of the world's population during the course of their lives and is currently one of the top ten causes for morbidity and mortality (Berton and Nestler, 2006; Nestler et al., 2002a). Approximately $30 \%$ of patients do not respond to available pharmaceutical agents for treating depression (Nestler et al., 2002b), and patients who do respond positively typically see changes only after weeks of treatment (Frazer and Benmansour, 2002). It has been reported that between 1992 and 2001, there was a $47 \%$ increase in emergency department visits due to suicide attempts and selfinflicted injuries in the United States (Larkin et al., 2008). Currently, healthcare professionals lack the necessary pharmacotherapies to adequately treat these patients in a timely and sufficient manner. A rapid acting antidepressant would therefore be of great utility to physicians, especially in emergency situations.

Recent studies have begun to investigate the antidepressant-like properties of ketamine through a variety of approaches (aan het Rot et al., 2010; Berman et al., 2000; Diazgranados et al., 2010a; Diazgranados et al., 2010b; Kollmar et al., 2008; Li et al., 2010b; Machado-Vieira et al., 2009; Paul et al., 2009; Popik et al., 2008; Price et al., 2009; Zarate et al., 2006). Ketamine is an N-methyl-Daspartate (NMDA) receptor antagonist and dissociative anesthetic, that has been shown to display rapid acting antidepressant activity in human clinical populations in several small cohort trials (aan het Rot et al., 2010; Berman et al., 2000; Diazgranados et al., 2010a; Diazgranados et al., 2010b; Zarate et al., 2006). These studies are especially significant because they focus on utilizing ketamine for patients with treatment resistant major depressive disorder who have failed on currently available therapeutics (aan het Rot et al., 2010; Diazgranados et al., 2010b; Zarate et al., 2006). In treatment resistant patients, therapeutic effects were observed within hours after ketamine administration, with effects lasting for up to seven days (Zarate et al., 2006). This has led to the belief that ketamine and similar compounds, such as dextromethorphan, may act as rapid acting antidepressants through the modulation of 
glutamatergic signaling (Krystal, 2007; Lauterbach, 2011; Machado-Vieira et al., 2009; Maeng and Zarate, 2007).

In addition to binding NMDA receptors and modulating glutamatergic signaling, ketamine interacts with several other protein targets, including sigma receptors (Hustveit et al., 1995; Seeman et al., 2009). Sigma receptors are modulatory proteins, of which two known subtypes exist, sigma-1 and sigma-2. Sigma-1 receptors are 223 amino acid proteins found at the mitochondrial-associated endoplasmic reticulum membrane (MAM) and can translocate to the plasma membrane. Additionally, sigma-1 receptors can exhibit chaperone activity and modulate inositol triphosphate $\left(\mathrm{IP}_{3}\right)$ receptor function, thereby effecting calcium signaling (Hayashi and Su, 2007b; Su et al., 2010b). Contrary to sigma-1 receptors, sigma-2 receptors remain poorly understood. They are smaller (18-22 kDa) than sigma-1 receptors and have been implicated in the regulation of cell proliferation and cell viability (Hellewell et al., 1994; Vilner and Bowen, 1993; Vilner et al., 1995).

Sigma receptors are considered to be protein targets for existing and novel antidepressant drugs (Fishback et al., 2010a; Hayashi and Su, 2008; Kulkarni and Dhir, 2009b). Currently utilized antidepressants such as selective serotonin reuptake inhibitors (SSRIs), monoamine oxidase inhibitors and tricyclic antidepressants bind to sigma receptors (Narita, 1996; Schmidt, 1989; Villard, 2011). In addition, various sigma receptor agonists display antidepressant-like activity in experimental animals (Matsuno et al., 1996; Pande et al., 1999; Skuza, 2003; Skuza and Rogoz, 2002, 2003; Ukai et al., 1998; Volz and Stoll, 2004; Wang et al., 2007b). There is also clinical trial data supporting sigma receptors as a viable antidepressant target, as the sigma receptor ligand igmesine hydrochloride has been shown to be as effective as the commonly prescribed SSRI fluoxetine at ameliorating depression symptoms (Pharmaprojects, 2004).

It is interesting to note that compared to conventional antidepressant drugs, the prototypic sigma receptor agonist (+)-pentazocine can elicit neuroadaptations in serotonergic neurons in the dorsal 
raphe nucleus that are measureable within two days of treatment compared to the two-week delay typical of marketed antidepressants (Bermack and Debonnel, 2001). This suggests that it may be possible to mediate fast acting antidepressant effects through sigma receptors.

In addition, sigma receptors modulate several signaling pathways and ion channels thought to be major elements in the etiology of depression (Fishback et al., 2010a; Hayashi and Su, 2008; Kulkarni and Dhir, 2009c). Sigma receptor modulation of these signaling pathways has also been shown to potentiate nerve growth factor (NGF)-induced neurite outgrowth in vitro, indicative of their potential to effect neuroplasticity and display antidepressant-like activity (Ishima et al., 2008; Nishimura et al., 2008; Takebayashi et al., 2002). Currently marketed antidepressant drugs can potentiate NGF-induced neurite outgrowth (Takebayashi et al., 2004), and it is believed that the delay in clinical efficacy of antidepressant medications may be related to the time required for neuronal remodeling to occur (Castren and Rantamaki, 2010; Duman et al., 1999; Duman et al., 2001).

Together, data in the literature suggest that sigma receptors may be capable of mediating the fast acting as well as delayed actions of antidepressant drugs. Therefore, to evaluate the potential involvement of sigma receptors in the antidepressant actions of ketamine, three types of studies were undertaken. First, the affinity of ketamine for each of the sigma receptor subtypes was determined. Second, the ability of ketamine to potentiate NGF-induced neurite outgrowth in rat pheochromocytoma (PC12) cells was determined, alone and in combination with a sigma receptor antagonist. Third, the involvement of sigma receptors in the in vivo antidepressant-like effects of ketamine using the forced swim test was also evaluated.

\subsection{Materials and Methods}

\subsubsection{Animals}


Male, Swiss Webster mice (24-32g; Harlan, Frederick, MD) were housed with food and water ad libitum, with a 12:12 hr light-dark cycle. All animals were housed in groups of five for at least one week prior to initiation of experiments at West Virginia University. All procedures were conducted as approved by the Institutional Animal Care and Use Committee at West Virginia University. The total number of animals utilized for the experiments was 210.

\subsubsection{Drugs and compounds}

Racemic ketamine was purchased from Sigma (St. Louis, MO). Imipramine hydrochloride was also obtained from Sigma (St. Louis, MO). Both BD1047 (N-[2-(3,4-dichlorophenyl)ethyl]-N-methyl-2(dimethylamino)ethylamine dihydrobromide) and NE-100 (N,N-dipropyl-2-(4-methoxy-3-(2phenylethoxy)phenyl)ethylamine hydrochloride) were purchased from Tocris (Ellisville, MO). Radioligands were sourced from PerkinElmer (Shelton, CT). All other chemicals and reagents were purchased from commercial suppliers (Sigma-Aldrich, St. Louis, MO).

\subsubsection{In vitro competition binding assays}

Rat liver $\mathrm{P}_{2}$ membrane ( $350 \mu \mathrm{g}$ protein) was used for all sigma receptor binding assays. Membrane homogenates were diluted in $50 \mathrm{mM}$ Tris- $\mathrm{HCL}$ buffer, $\mathrm{pH} 8.0$, and incubated in a total volume of $500 \mu \mathrm{L}$ with radioligand at $25^{\circ} \mathrm{C}$ in 96 well plates. The incubation time was 120 min for both

sigma-1 and sigma-2 assays. Sigma-1 receptors were labeled with $5 \mathrm{nM}\left[{ }^{3} \mathrm{H}\right](+)$-pentazocine and sigma-2 receptors were labeled with $3 \mathrm{nM}\left[{ }^{3} \mathrm{H}\right]$ di-o-tolylguanidine (DTG) in the presence of $300 \mathrm{nM}(+)$ pentazocine to block sigma-1receptors. Nonspecific binding was determined by the addition of haloperidol to a final concentration of $50 \mu \mathrm{M}$ (blanks). Ten concentrations of each test ligand were run in duplicates and each assay repeated three times. Following incubation and subsequent equilibrium, the samples were harvested, washed three times, and filtered through Unifilter GF/B filter plates (Perkin 
Elmer 50-905-1601, Shelton, CT) previously soaked in $0.5 \%$ polyethyleneimine. Bound radioactivity was counted using a Microbeta² 2450 Microplate Counter (Perkin Elmer, Waltham, MA).

\subsubsection{Neurite outgrowth assays}

Neurite outgrowth assays were performed as previously described with minor changes (Takebayashi et al., 2002). Briefly, PC12 cells were purchased from American Type Culture Collection (Manassas, VA) and grown in complete medium: RPMI1640 with 10\% horse serum, $5 \%$ fetal bovine serum, penicillin/streptomycin, all purchased from Invitrogen (Carlsbad, CA). Cells were passaged by dissociation from culture flasks with cold $\mathrm{Ca}^{2+}$ and $\mathrm{Mg}^{2+}$ free DPBS and resuspended in RPMI1640 with $5 \%$ fetal bovine serum to $1.0 \times 10^{4}$ cells/mL. For NGF titration experiments, NGF (Promega, Madison, WI) was added to final concentrations of $0.156,0.3125,0.625,1.25,2.5,5.0,10.0$ and $20.0 \mathrm{ng} / \mathrm{mL}$. For further neurite outgrowth experiments, NGF was added to a final concentration of $0.625 \mathrm{ng} / \mathrm{mL}$. Cells were plated at $1 \mathrm{~mL} /$ well in 24 well plates precoated with poly-D-lysine (PDL) and laminin (BD Falcon, San Jose, $\mathrm{CA}$ ) at $1 \mu \mathrm{g}$ per $\mathrm{cm}^{2}$. Plates were then incubated at $37^{\circ} \mathrm{C}$ for 36 to 48 hours. Images were captured to contain approximately 100 cells per field, with 3 images per well and were run in at least triplicate.

\subsubsection{Locomotor measurements}

Locomotor activity was measured utilizing an automated activity monitoring system (San Diego Instruments, San Diego, CA). Prior to locomotor activity measurements, animals were acclimated to the testing facility for at least $30 \mathrm{~min}$ and acclimated to the testing chambers for an additional $30 \mathrm{~min}$. Each testing chamber consisted of a plexiglass housing and a $16 \times 16$ photobeam array to detect lateral movements with a separate 16 photobeam array to detect rearing activity. Subsequent to the acclimation period, animals were treated and placed back in their respective chambers. Pretreatments 
were done 15 min prior to treatments with saline or ketamine. Locomotor activity was then quantified over a 30 min period. Ambulatory, fine and rearing movements were quantified in order to measure total locomotor activity. For some animals, immediately after the locomotor activity measurements, the mice were tested in the forced swim test (see section 2.6.).

For time course experiments, subsequent to the acclimation periods, animals were treated (i.p.) with either saline $(0.1 \mathrm{~mL} / 10 \mathrm{~g})$ or ketamine $(40 \mathrm{mg} / \mathrm{kg})$ and placed back into the locomotor chambers. Total locomotor activity was quantified over $30 \mathrm{~min}$. At 24, 48 and 72 hours animals underwent acclimation periods to both the facility and the testing chambers, then received saline injections and locomotor activity was quantified for a total of $30 \mathrm{~min}$. Immediately after each of the locomotor measurements, the animals were subject to the forced swim test (see section 2.2.7.).

\subsubsection{Forced swim test: acute measurements}

Mice were acclimated to the testing facility for $30 \mathrm{~min}$ prior to initiation of experiments. To evaluate the antidepressant-like effects of the compounds, mice received intraperitoneal (i.p.) injections with one of the following treatments: imipramine $(10-30 \mathrm{mg} / \mathrm{kg}, N=30)$, ketamine $(10-40 \mathrm{mg} / \mathrm{kg}, N=$ 30), BD1047 (5-20 mg/kg, $N=30), \operatorname{BD} 1047(5-20 \mathrm{mg} / \mathrm{kg})+$ ketamine $(40 \mathrm{mg} / \mathrm{kg}, N=30), \mathrm{NE}-100$ (1-5 $\mathrm{mg} / \mathrm{kg}, N=30), \mathrm{NE}-100(1-5 \mathrm{mg} / \mathrm{kg})+$ ketamine $(40 \mathrm{mg} / \mathrm{kg}, N=30)$ or saline $(0.1 \mathrm{~mL} / 10 \mathrm{~g}, N=10)$. Pretreatments were administered $15 \mathrm{~min}$ prior to receiving ketamine (40 mg/kg, i.p.). Thirty min after receiving their respective treatments, animals were placed in individual cylinders of water $(10 \mathrm{~cm}$ deep) for a total of $6 \mathrm{~min}$. The initial two min was an acclimation period and not scored. During the remaining four min, immobility time was quantified using ANY-Maze Version 4.63 video tracking software (Stoelting Co., Wood Dale, IL). Immobility was defined as no activity other than that required to maintain the animal's head above the surface of the water. ANY-Maze software settings were as follows: 
accustomization period $=120 \mathrm{sec}$, test duration $=240 \mathrm{sec}$, minimum immobility time $=2000 \mathrm{~ms}$, and immobility sensitivity $=75 \%$.

\subsubsection{Forced swim test: time course experiments}

Mice were acclimated to the testing facility for $30 \mathrm{~min}$ prior to initiation of experiments. Mice were administered (i.p.) either saline $(0.1 \mathrm{~mL} / 10 \mathrm{~g}, N=10)$ or ketamine $(40 \mathrm{mg} / \mathrm{kg}, N=10)$ on Day 1 of the experiment. Thirty min after receiving their respective treatments, animals underwent the forced swim test, as described above. All animals then received injections of saline (i.p.) at 24, 48 and 72 hours after the first administration, and underwent the forced swim test as described above at these time points.

\subsubsection{Data analysis}

Data from all experiments were analyzed using GraphPad Prism 4.0 (San Diego, CA). Data from the competition binding studies were analyzed using a nonlinear regression to determine the concentration of test ligand that inhibits $50 \%$ of the specific binding of the radioligand ( $\mathrm{IC}_{50}$ value). $\mathrm{K}_{\mathrm{i}}$ values were calculated using the Cheng-Prusoff equation (Cheng and Prusoff, 1973). The functional data were analyzed by either one-way analysis of variance (ANOVA) followed when applicable by post hoc Dunnett's or Tukey's multiple comparison tests, two-way ANOVA followed by Bonferroni's post hoc analysis, or unpaired two-tailed $t$-test. Data are represented as mean \pm S.E.M. $P<0.05$ was considered statistically significant for all data analyzed.

\subsection{Results}

\subsubsection{Competition binding assays}


As shown in Table 2.1, ketamine had micromolar affinity for sigma-1 and sigma-2 receptors. Imipramine, a tricyclic antidepressant, exhibited moderate nanomolar affinity for sigma-1 and sigma-2 receptors. NE-100 and BD1047, two sigma receptor antagonists, bound to sigma-1 and sigma-2 receptors with nanomolar affinities.

\begin{tabular}{|c|c|c|}
\hline Compound & sigma-1 $\mathbf{K}_{\mathbf{i}}$ & sigma-2 $\mathbf{K}_{\mathbf{i}}$ \\
\hline$( \pm)$-Ketamine & $139.60 \pm 6.13 \mu \mathrm{M}$ & $26.30 \pm 2.98 \mu \mathrm{M}$ \\
\hline Imipramine & $332.10 \pm 30.20 \mathrm{nM}$ & $327.20 \pm 33.30 \mathrm{nM}$ \\
\hline NE-100 & $7.20 \pm 0.94 \mathrm{nM}$ & $45.10 \pm 4.05 \mathrm{nM}$ \\
\hline BD1047 & $2.88 \pm 0.03 \mathrm{nM}$ & $26.40 \pm 2.63 \mathrm{nM}$ \\
\hline
\end{tabular}

Table 2.1. Affinities of various compounds for sigma receptors (both subtypes) used for both forced swim test and PC12 neurite outgrowth paradigms.

\subsubsection{Neurite outgrowth assays}

Maximal induction of neurite outgrowth was observed at a final concentration of $10 \mathrm{ng} / \mathrm{mL}$ of NGF $(25.44 \pm 1.81 \%)$. Therefore, for subsequent experiments, a final concentration of $0.625 \mathrm{ng} / \mathrm{mL}(5.65$ $\pm 1.09 \%$ ) was used, as this concentration did not significantly increase neurite outgrowth when compared to the lowest concentration tested $(F(7,64)=51.56, P<0.0001, q=1.87$, n.s.), and was low enough to allow for visualization of the potentiation of neurite outgrowth by compounds of interest (data not shown).

When the tricyclic antidepressant imipramine was tested for its ability to potentiate NGFinduced neurite outgrowth, a one-way ANOVA showed that the changes were significant $(F(6,56)=$ $35.05, P<0.0001)$. Furthermore, post hoc tests revealed that imipramine potentiated NGF-induced neurite outgrowth at the following concentrations: $10 \mathrm{nM}(q=3.04, P<0.05), 100 \mathrm{nM}(\mathrm{q}=7.00, P<$ 0.01), $1 \mu \mathrm{M}(q=12.09, P<0.01)$ and $10 \mu \mathrm{M}(q=4.56, P<0.01)$ (Figure 2.1.A.). 
A.

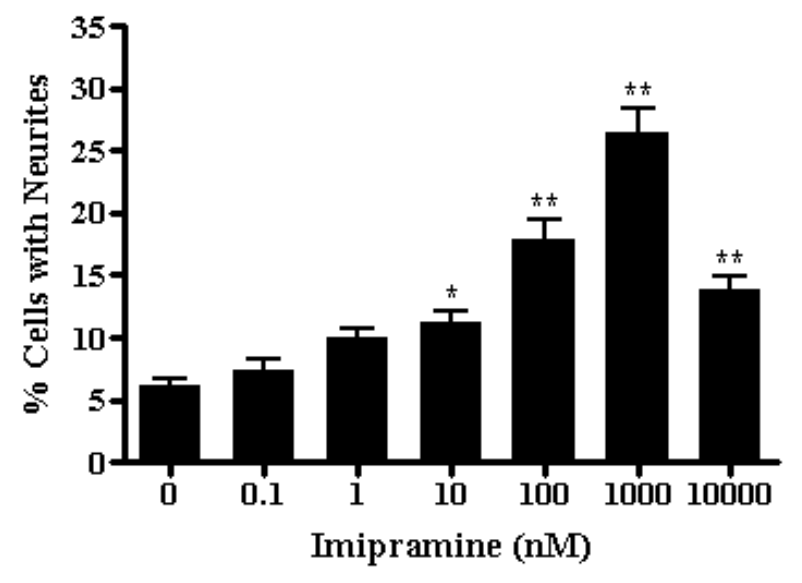

B.

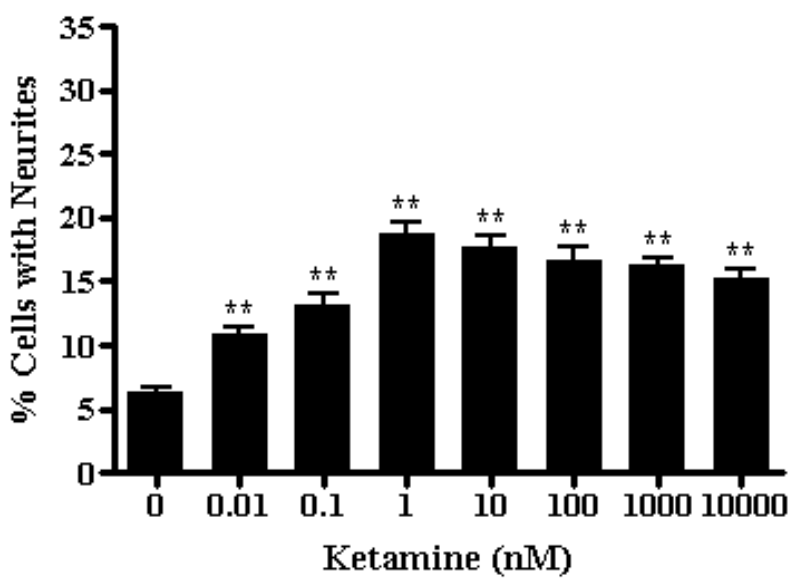

C.

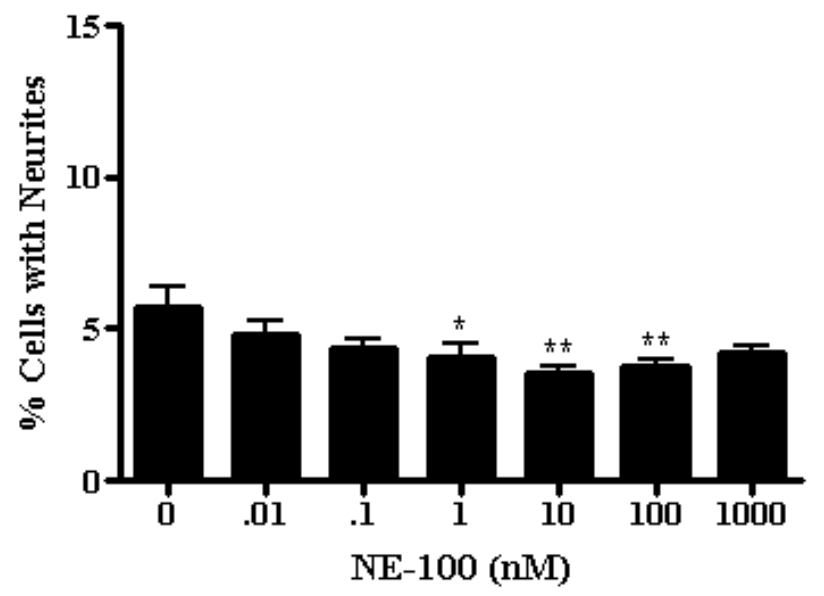

Figure 2.1. Effects of imipramine (0-10,000 nM; A), ketamine (0-10,000 nM) B), and NE-100 (0-1,000 nM) C) on NGF-induced neurite outgrowth. Data shown are expressed as mean \pm S.E.M. ${ }^{*} P<0.05, * * P<0.01$, $* * * P<0.001$; one way ANOVA followed by post hoc Dunnett's tests.

Ketamine also significantly potentiated NGF-induced neurite outgrowth $(F(7,64)=28.82, P<$ 0.0001). Ketamine significantly potentiated NGF-induced neurite outgrowth compared to vehicle controls at the following concentrations: $0.01 \mathrm{nM}(q=4.12, P<0.01), 0.1 \mathrm{nM}(q=6.36, P<0.01), 1 \mathrm{nM}$ $(q=11.41, P<0.01), 10 \mathrm{nM}(q=10.55, P<0.01), 100 \mathrm{nM}(q=9.49, P<0.01), 1 \mu \mathrm{M}(q=9.21, P<0.01)$ and $10 \mu \mathrm{M}(q=8.23, P<0.01)$ (Figure 2.1.B.). 
The sigma receptor antagonist, NE-100, exerted modest, but statistically significant, effects on NGF-induced neurite outgrowth on its own $(F(6,56)=3.35, P<0.01)$. NGF alone $(0.625 \mathrm{ng} / \mathrm{mL})$ stimulated neurite outgrowth in $5.70 \pm 0.68 \%$ of cells, as opposed to $4.10 \pm 0.44,3.50 \pm 0.29,3.70 \pm 0.21$ and $4.20 \pm 0.25 \%$ with NE-100 treatment of $1,10,100$ and $1000 \mathrm{nM}$, respectively. These changes in the percentage of NGF-induced neurite outgrowth as a result of NE-100 treatment, although minimal, were found to be significant when compared to vehicle controls: $1 \mathrm{nM}(q=2.85, P<0.05), 10 \mathrm{nM}(q=3.89, P$ $<0.01)$ and $100 \mathrm{nM}(q=3.46, P<0.01)$ (Figure 2.1.C.).

NE-100 also significantly decreased imipramine's potentiation of NGF-induced neurite outgrowth as shown by two-way ANOVA $(F(6,204)=10.61, P<0.0001$; Figure $2 A)$. Bonferroni's post hoc tests revealed that NE-100 treatment attenuated the ability of imipramine ( $100 \mathrm{nM}$ ) to increase neurite outgrowth at all concentrations tested: $10 \mathrm{nM}(t=6.98, P<0.001), 100 \mathrm{nM}(t=7.13, P<0.001)$ and 1000 $\mathrm{nM}(t=9.72, P<0.001)$. NE-100 also significantly attenuated the effects of imipramine $(1 \mu \mathrm{M})$ on neurite outgrowth at concentrations of $10 \mathrm{nM}(t=7.34, P<0.001), 100 \mathrm{nM}(t=8.81, P<0.001)$ and $1 \mu \mathrm{M}(t=$ 12.04, $P<0.001$ ) (Figure 2.2.A.).

A.

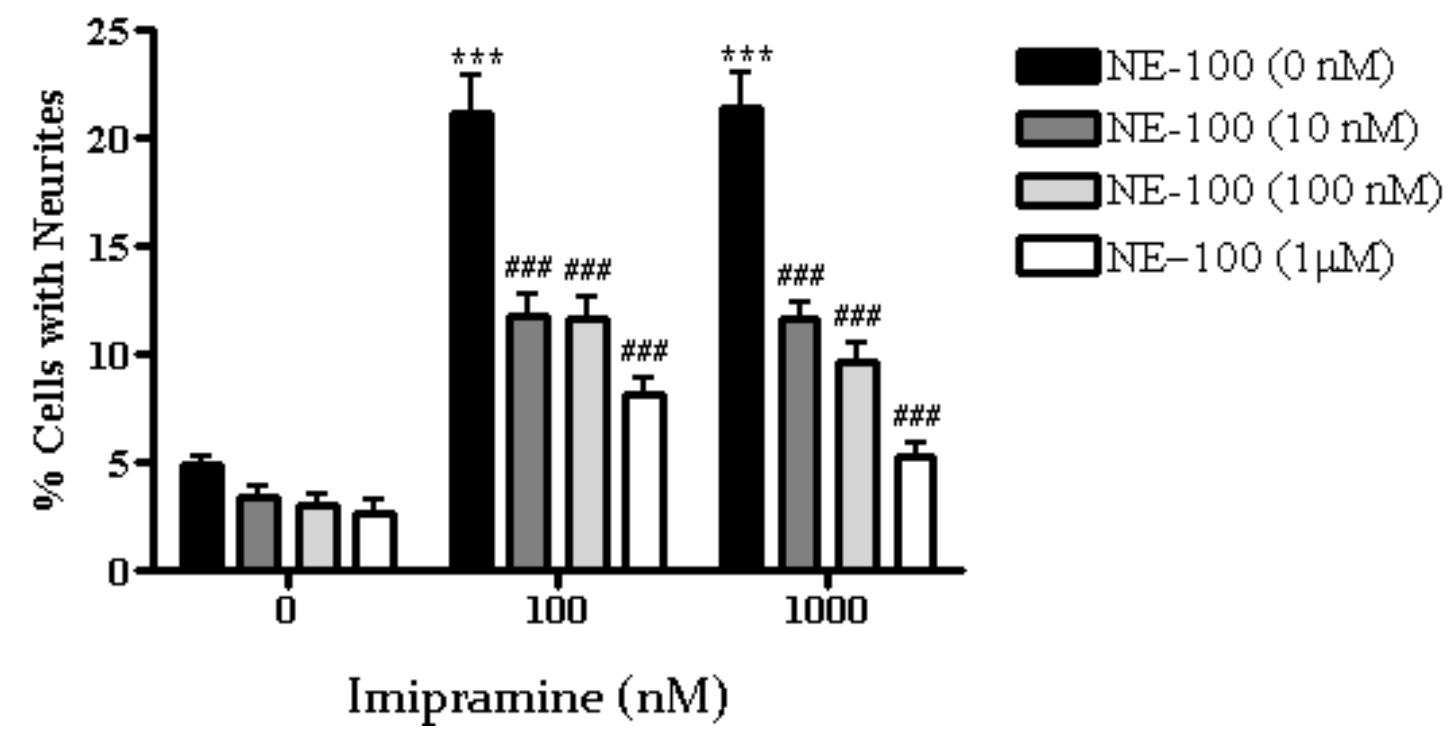


B.

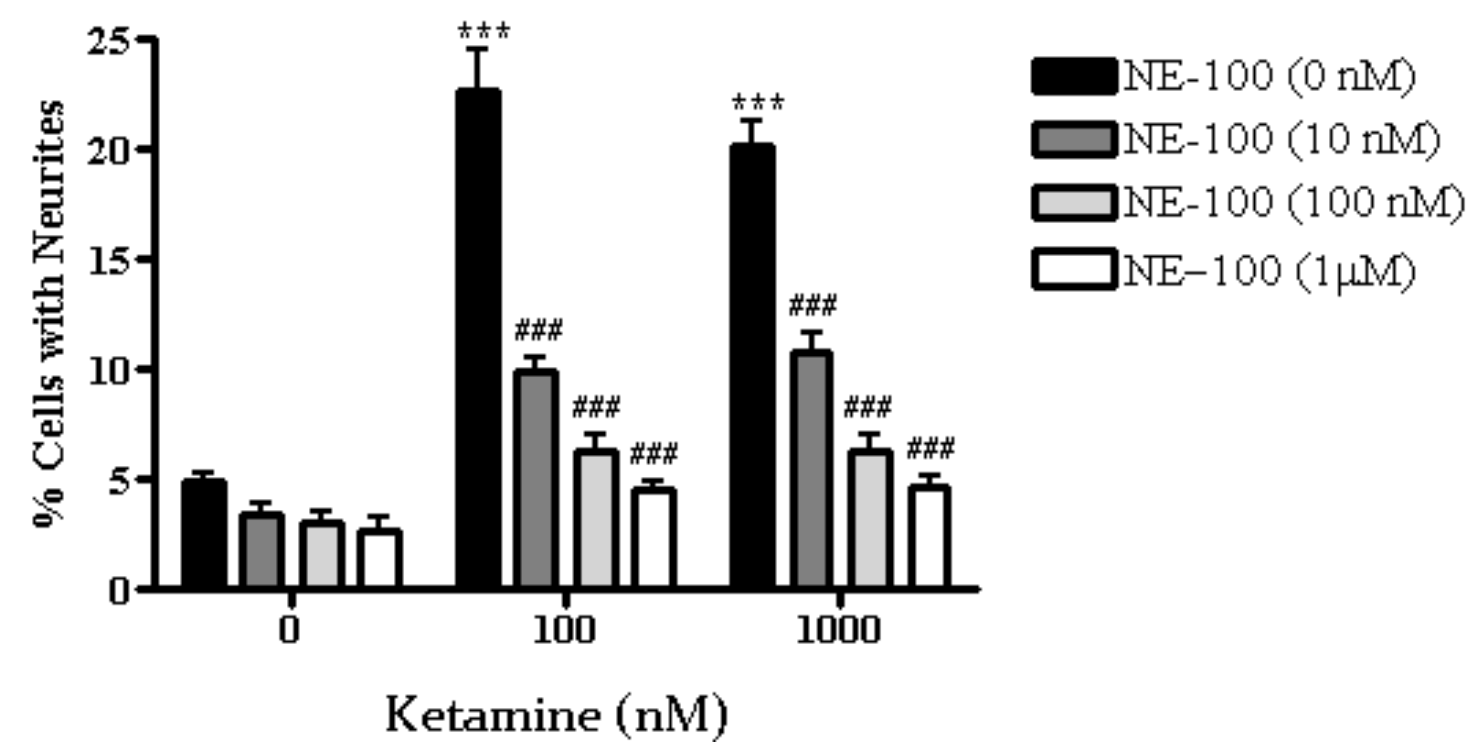

Figure 2.2. Effects of NE-100 (0-1000 nM) on NGF-induced neurite outgrowth potentiated by imipramine $(100$ and $1,000 \mathrm{nM}) \mathrm{A})$ and ketamine $(100$ and $1000 \mathrm{nM}) \mathrm{B})$. Data shown are expressed as mean \pm S.E.M. $* * * P<0.001, \# \# \# P<0.001$; one way ANOVA followed by post hoc Tukey's tests.

NE-100 (10 nM - $1 \mu \mathrm{M})$ had similar effects on the ability of ketamine to potentiate NGF-induced neurite outgrowth $(F(6,204)=21.46, P<0.0001)$. Post hoc Bonferroni's tests revealed that $10 \mathrm{nM}, 100$ $\mathrm{nM}$ and $1 \mu \mathrm{M}$ NE-100 significantly attenuated the ability of ketamine (100 nM) to potentiate NGFinduced neurite outgrowth $(t=10.83, P<0.001 ; t=13.86, P<0.001 ; t=15.38, P<0.001$, respectively) (Figure 2.2.B.). The effects of NE-100 pretreatment on the ability of ketamine ( $1 \mu \mathrm{M})$ to potentiate NGFinduced neurite outgrowth were similar to that of ketamine (100 nM) at $10 \mathrm{nM}(t=7.94, P<0.001), 100$ $\mathrm{nM}(t=11.80, P<0.001)$ and $1000 \mathrm{nM}(t=13.15, P<0.001)$.

\subsubsection{Locomotor activity}

Acute saline treatment resulted in a total locomotor activity count of $1621 \pm 275$ during the 30 min testing period. Acute ketamine $40 \mathrm{mg} / \mathrm{kg}$ treatment did not significantly alter locomotor activity compared to saline (1859 \pm 215 counts, $t=0.68$, n.s.) (data not shown). NE-100 alone displayed no significant effects on locomotor activity at any of the doses tested $(F(3,36)=0.06$, n.s. $)$. NE-100 $(1,2.5$ or 
$5 \mathrm{mg} / \mathrm{kg}$ ) in combination with ketamine $40 \mathrm{mg} / \mathrm{kg}$ did not significantly alter locomotor activity compared to saline ( $2464 \pm 438$ counts, $q=2.32$, n.s.; $2877 \pm 421$ counts, $q=3.45$, n.s.; $2919 \pm 412$ counts, $q=3.57$, n.s., respectively) or ketamine $40 \mathrm{mg} / \mathrm{kg}$ alone ( $q=1.66$, n.s.; $q=2.80$, n.s.; $q=2.91$, n.s., respectively).

When the effect of a single dose of ketamine $(40 \mathrm{mg} / \mathrm{kg})$ on locomotor activity over an extended time period was evaluated, it did not significantly alter locomotor activity when compared to saline at $0.5,24,48$ or 72 hours ( $1859 \pm 215$ vs. $1621 \pm 275$ counts, $q=0.95$, n.s.; $583 \pm 148$ vs. $1332 \pm 387$ counts, $q=2.98$, n.s.; $533 \pm 115$ vs. $850 \pm 233$ counts, $q=1.26$, n.s.; $1137 \pm 100$ vs. $1657 \pm 366$ counts, $q=2.07$, n.s., respectively) (data not shown).

\subsubsection{Forced swim test}

Saline administration exhibited an immobility time in the forced swim test of $107.4 \pm 6.1 \mathrm{sec}$. The tricyclic antidepressant drug imipramine significantly decreased immobility time in the forced swim test $(F(3,36)=8.08, P<0.0005)$. Post hoc Dunnett's tests revealed that imipramine 20 and $30 \mathrm{mg} / \mathrm{kg}$ significantly decreased immobility time when compared to saline $(33.1 \pm 15.8 \mathrm{sec}, q=4.07, P<0.01$; $31.7 \pm 6.9 \mathrm{sec}, q=4.08, P<0.01$, respectively) (Figure 2.3.A.). Ketamine administration also significantly reduced immobility time $(F(3,36)=8.73, P<0.0005)$, with ketamine $40 \mathrm{mg} / \mathrm{kg}$ displaying a marked decrease in immobility time compared to saline $(42.4 \pm 11.9 \mathrm{sec}, q=4.98, P<0.01)$ (Figure 2.3.B.). 
A.

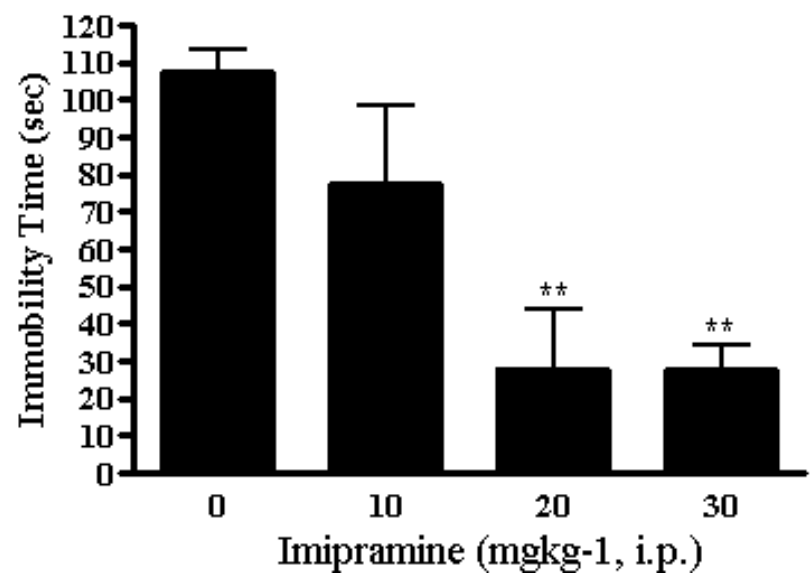

C.

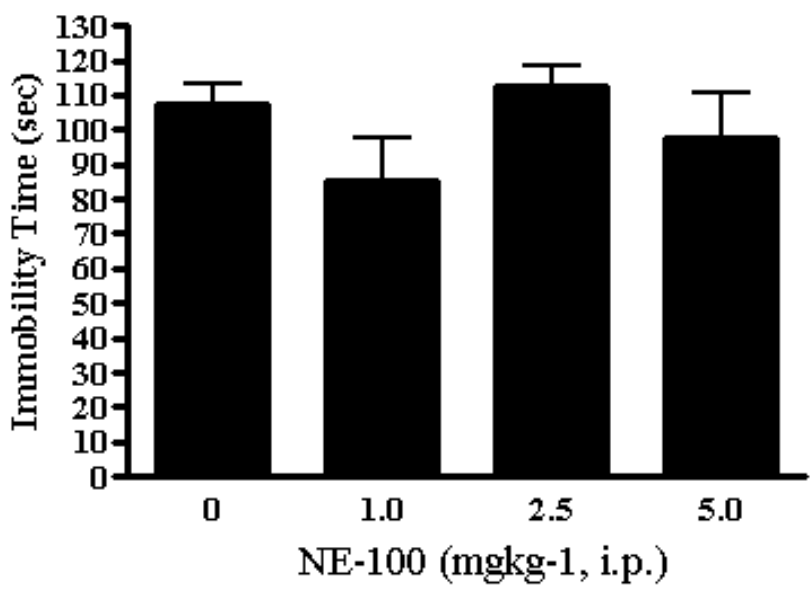

B.

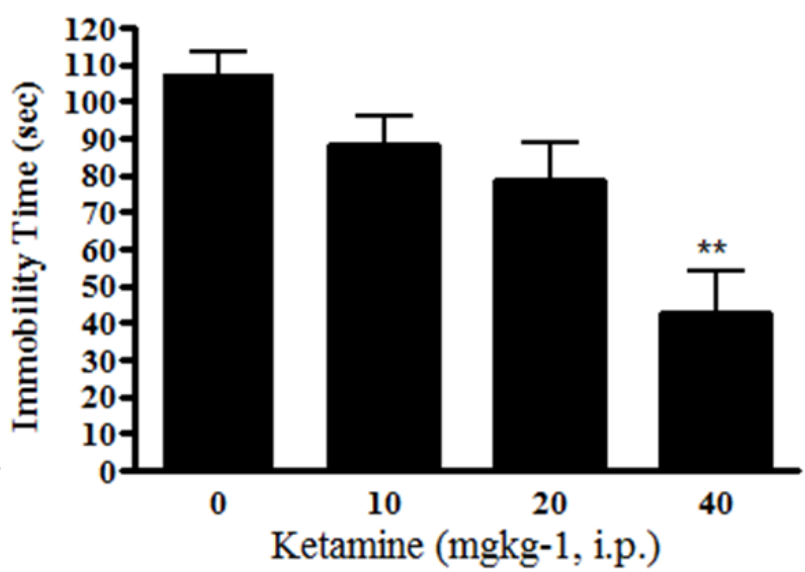

D.

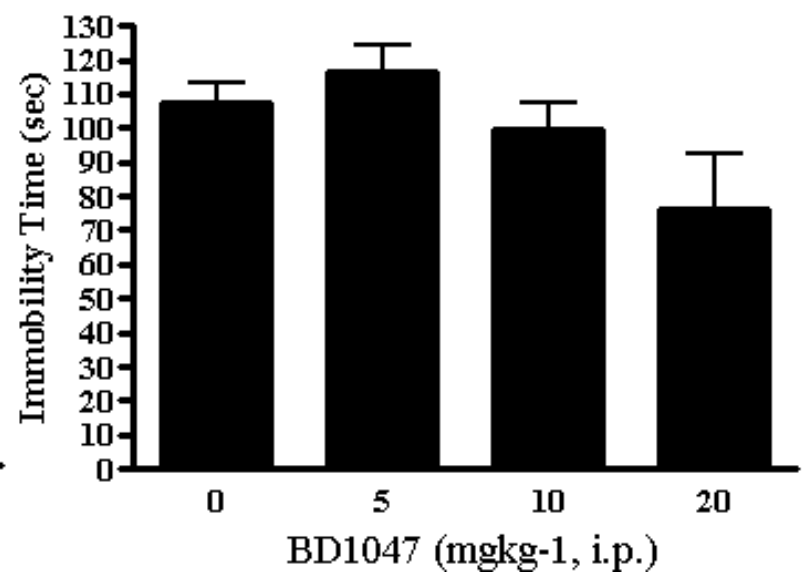

Figure 2.3. Antidepressant-like effects of imipramine $(0-30 \mathrm{mg} / \mathrm{kg}$, i.p.) A), ketamine $(0-40 \mathrm{mg} / \mathrm{kg}$, i.p.) B), $\mathrm{NE}-100$ (0-5 mg/kg, i.p.) C), and BD1047 (0-20 mg/kg, i.p.) D) in the mouse forced swim test. The tests started 30 min after an injection of saline or one of the four drugs. Data shown are expressed as mean \pm S.E.M. $(N=10) .{ }^{* *} P<0.01$; one way ANOVA followed by post hoc Dunnett's tests.

Two different sigma receptor antagonists, NE-100 (Figure 2.3.C.) and BD1047 (Figure 2.3.D.), displayed no significant effects in the forced swim test $(F(3,36)=1.42$, n.s.; $F(3,36)=2.85$, n.s., respectively).

BD1047 5, 10 or $20 \mathrm{mg} / \mathrm{kg}$ pretreatment was unable to attenuate the antidepressant-like effects of ketamine $40 \mathrm{mg} / \mathrm{kg}$ when analyzed by post hoc Tukey's multiple comparison tests $(q=1.22$, n.s.; $q=$ 0.86 , n.s.; $q=1.77$, n.s., respectively) (Figure 2.4.A.). Similarly, as shown in Figure 2.4.B., NE-100 
pretreatment was unable to attenuate the antidepressant-like effects of ketamine $40 \mathrm{mg} / \mathrm{kg}$ at any of the following doses: $1 \mathrm{mg} / \mathrm{kg}(q=0.45$, n.s.), $2.5 \mathrm{mg} / \mathrm{kg}(q=0.80$, n.s.) or $5 \mathrm{mg} / \mathrm{kg}(q=0.69$, n.s. $)$.

A.

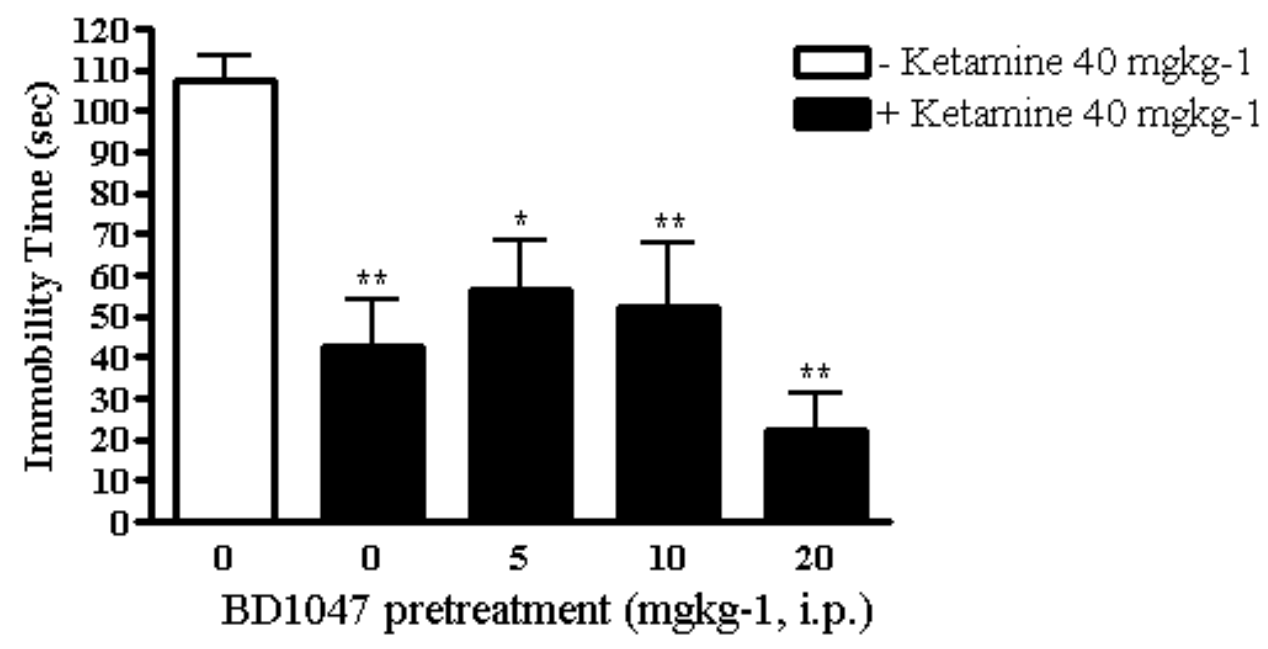

B.

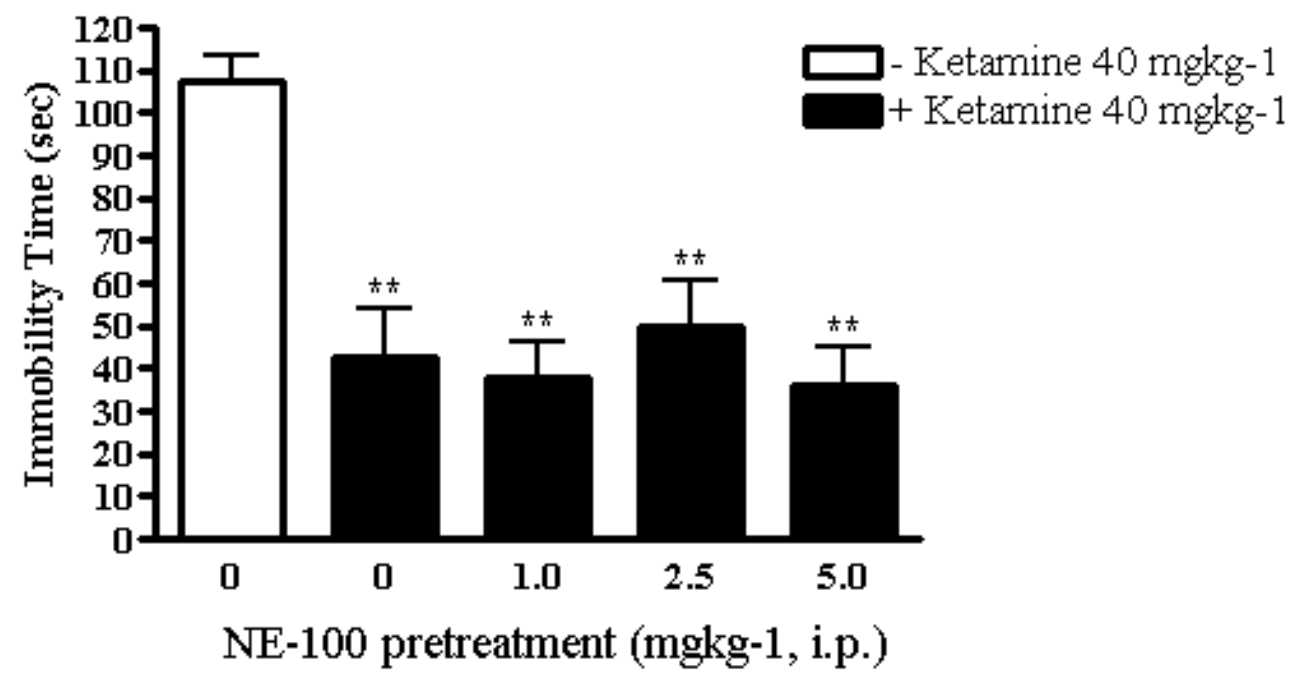

Figure 2.4. Antidepressant-like effects of BD1047 (0-20 mg/kg, i.p.) + Ketamine (40 mg/kg, i.p.) A) and $\mathrm{NE}-100$ (0-5 mg/kg, i.p.) + Ketamine (40 mg/kg, i.p.) B) in the mouse forced swim test. The mice were injected ketamine 15 min after the BD1047 or NE-100 pretreatment. The tests were started 30 min after the injection of ketamine or saline. Data shown are expressed as mean \pm S.E.M. $(N=10) .{ }^{*} P<0.05$, ${ }^{* *} P<0.01$; one way ANOVA followed by post hoc Tukey's tests.

\subsubsection{Time course of the antidepressant-like effects of ketamine}


One-way ANOVA revealed that a single administration of ketamine $40 \mathrm{mg} / \mathrm{kg}$ produced effects in the repeated forced swim test $(F(7,72)=7.18, P<0.0001)$. Post hoc Dunnett's analysis revealed that ketamine $40 \mathrm{mg} / \mathrm{kg}$ significantly decreased immobility time $30 \mathrm{~min}$ after administration compared to saline administration $(32.1 \pm 8.9 \mathrm{sec}, q=2.89, P<0.05)$. However, this effect was not present 24,48 or 72 hours post-administration $(91.0 \pm 10.8 \mathrm{sec}, q=0.39$, n.s.; $124.4 \pm 14.5 \mathrm{sec}, q=2.25$, n.s.; $130.7 \pm 13.1$ sec, $q=2.61$, n.S., respectively) (Figure 2.5.).

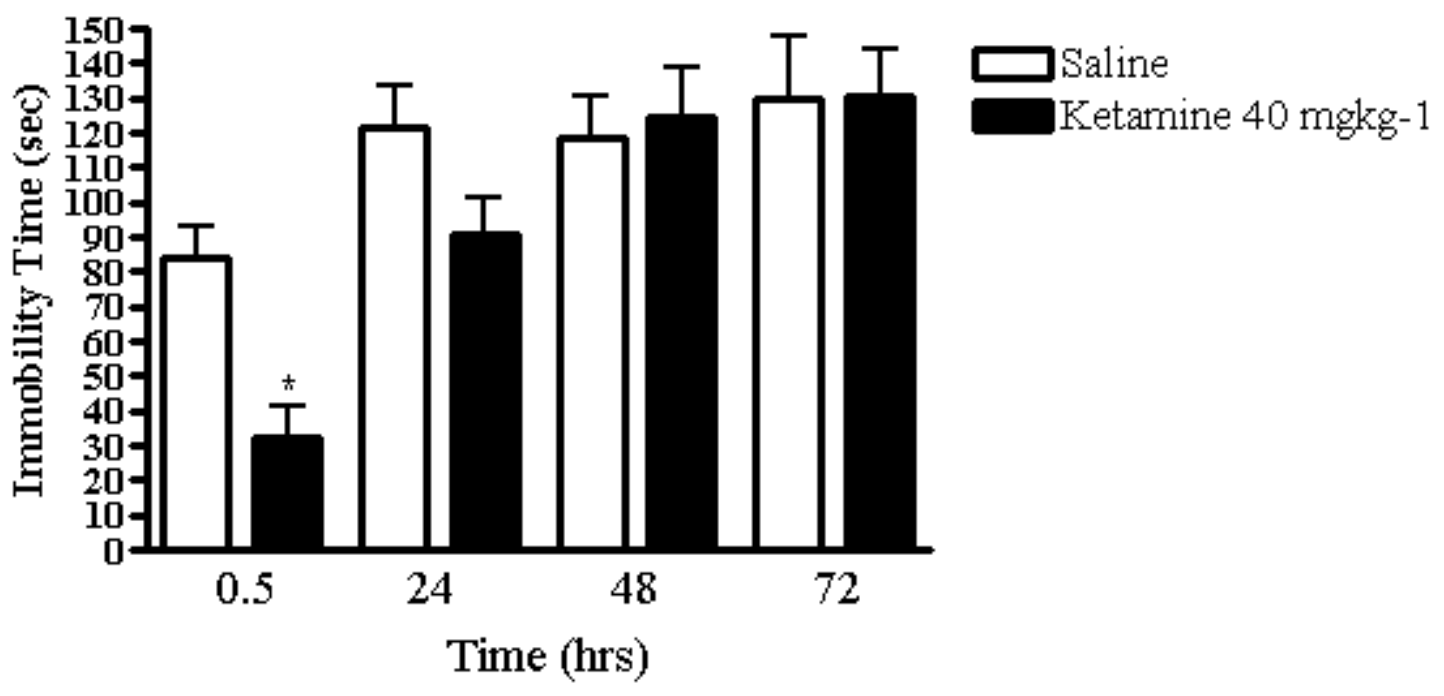

Figure 2.5. Time course of antidepressant-like effects of ketamine $(40 \mathrm{mg} / \mathrm{kg}$, i.p.) in the mouse forced swim test. The test started $0.5,24,48$ and $72 \mathrm{~h}$ after the injection of saline or ketamine. Data shown are expressed as mean \pm S.E.M. $(N=10)$. ${ }^{*} P<0.05$; one way ANOVA followed by post hoc Dunnett's tests.

\subsection{Discussion}

This study is the first to provide binding affinities of ketamine for both sigma receptor subtypes, in addition to determining the implications of this interaction on the antidepressant-like effects of ketamine in vitro and in vivo.

Ketamine binds to both subtypes of sigma receptors in the micromolar range. In addition to sigma receptors and NMDA receptors, ketamine has been shown to interact with HCN1 channels, dopamine $\mathrm{D}_{2}$ receptors, and opioid receptors (Chen, 2009; Hirota et al., 1999; Hustveit et al., 1995; Seeman et al., 2009). This pattern of binding suggests that ketamine may produce direct effects through 
sigma receptors, but that other mechanisms of action are also likely to contribute. Further, it is possible that the cumulative actions of ketamine through multiple targets in the body may be modified downstream through sigma receptor-mediated interventions given the modulatory role of sigma receptors in cellular functions (Hayashi et al., 2000b; Hayashi and Su, 2007a; Nishimura et al., 2008).

This is also the first report of ketamine potentiating NGF-induced neurite outgrowth in PC12 cells, an effect that is mitigated through administration of a sigma receptor antagonist. Recent studies have demonstrated that sigma receptor agonists potentiate NGF-induced neurite outgrowth in this cellular model, whereas antagonists or inverse agonists have no effect or decrease NGF-induced neurite outgrowth (Hashimoto et al., 2007a; Kishimoto, 2010; Nishimura et al., 2008; Takebayashi et al., 2002; Villard, 2011). In addition, several currently utilized antidepressants that bind to sigma receptors have been shown to potentiate NGF-induced neurite outgrowth (Hashimoto et al., 2007a; Nishimura et al., 2008; Takebayashi et al., 2002). The ability of ketamine to potentiate NGF-induced neurite outgrowth suggests that, similar to other antidepressant drugs, it can stimulate neuronal remodeling and this effect may underlie the observed therapeutic effects. With conventional antidepressant drugs, this remodeling is thought to be associated with the delay in therapeutic efficacy and may thus correspond to the persistent antidepressant effects observed with ketamine, even after the drug has been eliminated from the body (Berman et al., 2000; Duman et al., 1997; Duman et al., 1999; Zarate et al., 2006).

Ketamine appears to exert its potentiating effects on NGF-induced neurite outgrowth by interacting with sigma receptors, thereby modulating pathways relevant to neuroplasticity. Agonist activity at sigma receptors would explain the ability of ketamine to potentiate NGF-induced neurite outgrowth and also explain the ability of NE-100 to dose-dependently attenuate this effect. Further supporting the theory of direct interactions between ketamine and sigma receptors, are results from imaging studies displaying competition binding between racemic ketamine and a $\left[{ }^{11} \mathrm{C}\right]$ labeled sigma receptor PET ligand, SA5845 in numerous brain regions in primates (Kortekaas et al., 2008). 
In addition, sigma receptors may play a modulatory role in signaling pathways activated by ketamine administration that are relevant to neurite outgrowth. It has been shown that activation of sigma receptors can potentiate signaling through ERK/MAPK and $\mathrm{IP}_{3} / \mathrm{Akt}$ pathways, both of which are implicated in neurite outgrowth (Nishimura et al., 2008). Sigma-1 receptors have been shown to stabilize $\mathrm{IP}_{3}$ receptors at the MAM and ultimately increase $\mathrm{Ca}^{2+}$ release through this stabilization (Hayashi et al., 2000a; Hayashi and Su, 2007b; Su et al., 2010b). The ability of sigma-1 receptors to stabilize $\mathrm{IP}_{3}$ receptors may result in increased $\mathrm{Ca}^{2+}$ efflux from $\mathrm{IP}_{3}$ receptors and result in the potentiation of signaling pathways required for neurite outgrowth in this cellular model (Nishimura et al., 2008). Recently, it was reported that the antidepressant effects of ketamine involve mammalian target of rapamycin (mTor) signaling through ERK and Akt pathways (Li et al., 2010b). It was shown that ketamine increased the population of mushroom spines and increased spine density in medial prefrontal cortex (mPFC) pyramidal neurons, and this was attenuated by infusion of an mTor inhibitor, rapamycin (Li et al., 2010b). Sigma receptor ligands have been shown to regulate Akt signaling, leading to the modulation of NGF-induced neurite outgrowth (Li et al., 2010a; Nishimura et al., 2008). This could be another possible explanation as to how the sigma receptor antagonist NE-100 is modulating the effects of ketamine on NGF-induced neurite outgrowth. The determination as to whether ketamine is acting as a sigma agonist through direct interactions with the receptor and/or whether sigma receptors are modulating pathways relevant to ketamine's effects on neurite outgrowth in PC12 cells has yet to be made.

It has been speculated that the antidepressant effects of ketamine are mediated by increases in a-amino-3-hydroxy-5-methyl-4-isoxazolepropionic acid (AMPA) influx (Li et al., 2010b; Maeng et al., 2008). Treatment with 2,3-dihydroxy-6-nitro-7-sulfamoyl-benzo[f]quinoxaline-2,3-dione (NBQX), an AMPA antagonist, completely blocked the induction of the phosphorylation of Akt and ERK in response to ketamine treatment in the MPFC (Li et al., 2010b). The effects of AMPA signaling on the antidepressant effects of ketamine have also been shown to be important in behavioral paradigms. 
Results linking the antidepressant-like effects of ketamine to AMPA receptors, by using NBQX to attenuate the antidepressant-like effects of ketamine in the forced swim test have been reported (Maeng et al., 2008). Rapamycin has also been shown to attenuate the antidepressant-like effects ketamine in the forced swim test (Li et al., 2010b).

The current study used the forced swim test to show that the behavioral antidepressant-like effects of ketamine are not mediated by its interaction with sigma receptors. This conclusion is substantiated by the use of two well-established sigma receptor antagonists that were unable to attenuate the antidepressant-like effects of ketamine at any dose tested in this paradigm. In contrast, these antagonists have been reported to attenuate the antidepressant-like actions of sigma receptor agonists in the forced swim test (Wang et al., 2007b). It is believed that the antidepressant effects of sigmareceptor agonists are primarily the result of their interactions with sigma-1 receptors (Kulkarni and Dhir, 2009c). NE-100 and BD1047, two well accepted sigma-1 receptor antagonists which also exhibit significant affinity and putative antagonist effects at sigma-2 receptors (McCracken et al., 1999; Vilner and Bowen, 2000), were shown in the current study to not attenuate the antidepressant-like effects of ketamine. It therefore appears that increases in AMPA signaling are required for the acute antidepressant-like behavioral effects of ketamine, and these signaling events occur through sigma receptor independent mechanisms, which include alterations in mTOR (Li et al., 2010b).

Our study also further validates that the forced swim test is a valuable tool for the study of the antidepressant-like actions of ketamine, and it has been reported in rats that these effects are not confounded by general increases in activity levels (Engin et al., 2009). It has also been shown that acute treatment with ketamine $(50 \mathrm{mg} / \mathrm{kg}$ ) does not increase exploratory or general locomotor activity (Popik et al., 2008). The current study provides further evidence that the antidepressant-like effects of ketamine in animal models such as the forced swim test are not confounded by alterations in locomotor 
activity. It should also be noted that in this study, a single administration of ketamine did not alter locomotor activity when compared to saline for up to 72 hours.

In the current study, we found no evidence of persistent antidepressant-like effects by a single administration of ketamine in repeated trials of the forced swim test. These results are similar in nature to a previous report (Popik et al., 2008), and another recent study has shown that in CD-1 and Balb/cJ mice, ketamine displays no long-term antidepressant-like actions one week after administration in the forced swim test (Bechtholt-Gompf, 2011). However, there is variation between reported studies with regard to the persistent effects of ketamine in animal models of antidepressant-like behaviors. It has been reported that two weeks after administration, the antidepressant-like effects of ketamine were still present in the forced swim test (Maeng et al., 2008), and another group has also previously published results showing that an anesthetic dose of ketamine $(160 \mathrm{mg} / \mathrm{kg})$ was able to decrease immobility time in the forced swim test 10 days post-administration (Yilmaz et al., 2002). The methodologies used to determine whether ketamine had sustained antidepressant-like effects in each of these studies were drastically different and potentially led to the differences seen in previously reported studies (Maeng et al., 2008; Popik et al., 2008; Yilmaz et al., 2002).

The behavioral results of antidepressant-like effects presented in this report were obtained through the use of a single assay, the forced swim test. Therefore, it will be important in future studies to validate the pattern of effects presented here using other behavioral paradigms such as learned helplessness, chronic mild stress, tail suspension tests and social defeat stress models (Martin and Brown, 2010; Yan et al., 2010). Although the effects of ketamine treatment in a variety of these models have begun to be determined, the role that sigma receptors play in these effects has yet to be studied (Koike et al., 2011; Li et al., 2011). In addition, the role of gender and other physiologic characteristics in many of these behavioral models of depression and antidepressant-like effects is yet unknown (Martin and Brown, 2010). 
In conclusion, the data presented here show for the first time that ketamine potentiates NGF-

induced neurite outgrowth in an in vitro model and provides evidence that this effect occurs through a sigma receptor dependent mechanism. Additional studies to understand the molecular signaling events by which these effects occur are warranted and may shed light on the mechanism by which ketamine displays antidepressant actions.

\subsection{Contributions}

Matthew Robson conducted all in vivo studies and ex vivo radioligand binding studies. Michael Seminerio, Ph.D. aided in conducting radioligand binding studies. Meenal Elliott, Ph.D. and Matthew Robson performed and analyzed neurite outgrowth assay experiments.

This chapter has previously been published as:

Robson MJ, Elliott M, Seminerio MJ, Matsumoto RR. Evaluation of sigma $(\sigma)$ receptors in the antidepressant-like effects of ketamine in vitro and in vivo. European

Neuropsychopharmacology. 2012. 22(4): 308-317. PubMed ID: 21911285 


\begin{abstract}
The Involvement of Sigma Receptor Modulation in the Neurotoxic Actions of Methamphetamine
\end{abstract}

\title{
Matthew J. Robson
}

Methamphetamine (METH) is an illicit, addictive psyhostimulant and chronic use of the drug is known to result in striatal neurotoxicity that has been linked to cognitive deficits and an increased risk of developing Parkinson's disease. The precise molecular mechanisms by which these effects occur have yet to be fully delineated, however it is known that METH results in the induction of endoplasmic reticulum (ER) stress, reactive gliosis and neuroinflammation in regions subject to the neurotoxic actions of the drug. In addition to interacting with monoaminergic targets with the CNS, METH binds to both subtypes of sigma receptors at physiologic concentrations and sigma receptor antagonists have been shown to attenuate many behavioral and physiologic effects of METH in vivo, including neurotoxicity. Additionally, sigma receptor antagonists have been shown to block the toxic effects of the drug in vitro. The determination as to whether sigma receptor modulation alters METH-induced ER stress, reactive gliosis or neuroinflammation however has yet to be made. The purpose of the studies contained herein were therefore to determine if sigma receptor ligands mitigate the effects of METH on ER stress, reactive gliosis and neuroinflammation using various in vitro and in vivo models. METH treatment resulted in a significant upregulation of PERK-mediated ER stress specific genes including atf3, atf4, chop and gadd34 after 6, 12 and $24 \mathrm{hrs}$ using an in vitro model of METH neurotoxicity, an effect that was unable to be mitigated by treatment with SN79, a sigma receptor antagonist. In vivo studies revealed that the ability of sigma receptor antagonists to attenuate METH-induced dopaminergic neurotoxicity is correlated with a mitigation of METH-induced hyperthermia. Additionally, METH treatment was found to result in striatal glial cell activation and neuroinflammation in male, Swiss Webster mice, effects that were blocked by SN79 treatment. Results from the current studies provide evidence that sigma receptor antagonists such as SN79 are modulating neuroinflammation stemming from METH, thereby conveying protective effects against METH-induced neurotoxicity. 


\section{Chapter 3}

\section{Introduction}

Methamphetamine Abuse and Neurotoxicity 


\subsection{Drug Abuse}

In the United States alone, drug abuse is responsible for billions of dollars in costs associated with treatments for addiction, as well as various medical complications that develop as a result of acute and chronic substance abuse (Policy, 2004). In addition, incarceration and health care costs associated with emergency department admissions from drug overdose are problematic (Rich et al., 2011). Compounding this problem, several classes of currently abused substances lack FDA-approved pharmacotherapies to treat the addiction, overdose and toxic effects associated with their usage (Jupp and Lawrence, 2010). New strategies and ultimately, therapies developed for treating the effects of drugs of abuse are essential. Classical targets, including the dopaminergic system, have not yielded clinically viable treatments capable of ameliorating the acute physiological or the rewarding effects of drugs such as cocaine and METH, which target these systems (Jupp and Lawrence, 2010). These results have led to the current state which involves a myriad of research dedicated toward the discovery and development of novel strategies for the treatment of substance abuse.

\subsection{Sigma Receptors and Drug Abuse}

Sigma receptors have recently become compelling targets for the development of novel therapeutics aimed at treating the effects of a variety of abused substances. Of the two subtypes, sigma1 and sigma-2, which are described in detail in other articles in this volume, sigma-1 receptors have garnered increasing attention as accumulating knowledge about their functions have emerged. Sigma-1 receptors are expressed in several regions of the brain that have implications in drug abuse and addiction (i.e. limbic regions such as the hippocampus and striatal regions such as the caudate, putamen, nucleus accumbens and amygdala) (Alonso et al., 2000; Phan, 2000).

Sigma receptors were initially theorized to be novel targets for treating addiction disorders after it was shown that various drugs of abuse interact with these receptors at physiologically relevant 
concentrations. Drugs of abuse previously reported to interact directly with both subtypes of sigma receptors include: cocaine and certain metabolites of cocaine; METH; 3,4-

methylenedioxymethamphetamine (MDMA) and phencyclidine (PCP) (de Costa et al., 1989; de Costa et al., 1992; Liu et al., 2005; Matsumoto et al., 2001a; Sharkey et al., 1988; Weber et al., 1986). In addition to direct interactions with sigma-1 receptors, the modulation of neurotransmitter systems and signaling pathways relevant to the actions of many of the above drugs of abuse are believed to involve sigma-1 receptors (Gonzalez-Alvear and Werling, 1994, 1995; Gonzalez and Werling, 1997; Gudelsky, 1995; Kobayashi et al., 1997; Su and Hayashi, 2003; Weatherspoon et al., 1996). Sigma-1 receptors also have profound effects on drug-induced gene expression, including genes that are related to the behavioral effects of abused substances (Hayashi and Su, 2005; Liu et al., 2005; Liu and Matsumoto, 2008) and learning and memory processes (Yang et al., 2009). The evidence that sigma-1 receptors can modulate diverse processes necessary for the effects of a variety of drugs of abuse makes them viable targets for the development of novel drugs aimed at treating the negative side effects of several drugs of abuse, including addiction.

\subsection{Methamphetamine (METH)}

METH is an addictive psychostimulant that targets monoaminergic systems within the central nervous system (CNS). It is a very widely abused substance worldwide with an estimated 25 million users and is currently the second most abused substance in the world, behind only Cannabis (Cadet and Krasnova, 2009; Rawson and Condon, 2007). Inexpensive production, long half-life and low cost are all associated with its popularity among users (Cadet and Krasnova, 2009). Emergency room admissions stemming from METH usage have also risen in previous years. In the period from 1992 to 2002, METH related ER admissions rose from 10 to 52 per 100,000 people (Roehr, 2005). Clearly, METH abuse and 
overdose is a significant public health problem, one in which there are currently no pharmacologic treatments approved by the FDA (Jupp and Lawrence, 2010).

METH abuse is associated with several negative effects including tachycardia, increased blood pressure, hyperthermia, stroke, insomnia, anxiety, hallucinations, paranoia, memory loss, depression, dental decay, psychosis, weight loss, and with extended usage significant neurotoxicity (Romanelli and Smith, 2006). Two of these side effects, hyperthermia and neurotoxicity are particularly problematic. METH overdose can result in life threatening hyperthermia that raises core body temperature in excess of $41^{\circ} \mathrm{C}$ and can result in renal and liver damage (Cadet and Krasnova, 2009; Romanelli and Smith, 2006). The neurodegenerative effects of chronic METH abuse are hypothesized to be responsible for deficits in attention, memory and executive functioning in METH addicts (Gonzalez et al., 2007; Gonzalez et al., 2004; Hart et al., 2012; Woods et al., 2005). Additionally, it has been hypothesized for some time that chronic METH usage leading to neurotoxicity may result in an increased risk of developing Parkinson's disease (Guilarte, 2001; Volkow et al., 2001b). Recently, it was found that METH abusers do indeed have an increased risk of developing Parkinson's disease later in life as opposed to non-METH using controls (Callaghan et al., 2010; Callaghan et al., 2012; Kuehn, 2011). With the numbers of METH abusers worldwide, the increased risk of developing Parkinson's disease in these individuals is a significant public health concern. Exacerbating this problem is the current lack of any approved pharmacotherapies aimed at treating METH-induced neurotoxicity.

The hypothesis that METH-induced neurotoxicity may lead to an increased risk of developing Parkinson's disease stemmed from studies showing that METH-induced neuronal damage affects dopaminergic nerve terminals in related regions of the brain as Parkinson's disease. Imaging studies have been utilized to show a reduction in dopamine transporters (DAT) in the cortex and striatum of chronic METH users, indicative of nerve terminal damage (Sekine et al., 2001; Sekine et al., 2003; Volkow et al., 2001a; Volkow et al., 2001b). Additionally, a reduction in serotonin transporters (SERT) in 
humans has also been reported in the cortex, indicative of damage to serotonergic nerve terminals (Sekine et al., 2006).

Damage to dopaminergic and serotonergic nerve terminals by METH has been shown in rodent models by using a variety of administration regimens (Albers and Sonsalla, 1995; Bowyer et al., 1994; Brunswick et al., 1992; Krasnova et al., 2010; Matsumoto et al., 2008; Schwendt et al., 2009; Seminerio et al., 2011). Additionally, a myriad of potential therapies aimed at counteracting these effects have also been used in rodents and have been reviewed elsewhere (Escubedo et al., 2009; Krasnova and Cadet, 2009; Panenka et al., 2012; Rodvelt and Miller, 2010).

\subsection{METH-Induced Neurotoxicity: Potential Mechanisms}

The nerve terminal damage elicited by METH is believed to occur through a variety of cellular and molecular mechanisms involving various cell types within the CNS. Figure 3.1 depicts a simplified dopaminergic nerve terminal with highlighted mechanisms by which METH is believed to result in neurotoxicity. Some of the mechanisms by which METH is believed to result in neurotoxicity are listed below:

1. Dopamine and serotonin release: Excess release of these two neurotransmitters (primarily dopamine) is believed to be the initiating event leading to neurotoxicity elicited by METH (Riddle et al., 2006; Stephans and Yamamoto, 1994). This excess release can lead to post-synaptic signaling involved in cellular toxicity (Cadet and Krasnova, 2009; Gross et al., 2011). Additionally, excess dopamine is known to result in reactive forms of dopamine called dopamine quinones (DAQ) that are believed to be major contributors to METH-induced neurotoxicity (Kuhn et al., 2006; LaVoie and Hastings, 1999a, b; Miyazaki and Asanuma, 2009).

2. Excitotoxicity: Glutamatergic excitotoxicity is also implicated in the neurotoxic actions of METH. The exact mechanism by which occurs is currently unknown, however it is known that METH 
results in glutamate release in the CNS and glutamate antagonists have been previously shown to mitigate some of the neurotoxic actions of METH (Battaglia et al., 2002; Cadet and Krasnova, 2009; Sonsalla et al., 1989; Tata and Yamamoto, 2008). Increases in glutamate may originate from many sources including the modulation of glutamate uptake by astrocytes (Coulter and Eid, 2012), as METH is known to affect astrocyte function (see below). Glutamate is hypothesized to result in nitric oxide formation as a result of METH, an effect that can result in neuronal damage (Cadet and Krasnova, 2009).

3. Glial cell activation: METH treatment also results in the activation of glial cells within the CNS, including microglial cells and astrocytes (Cadet and Krasnova, 2009). METH has been shown to result in microglial activation in human abusers and several groups have published reports providing evidence that METH results in the activation of astrocytes and microglial cells in preclinical models (Kelly et al., 2012; Sekine et al., 2008; Thomas et al., 2004a; Thomas et al., 2004c). It is also hypothesized that glial cell activation results in cytokine and ROS/RNS release that mediates several aspects of toxicity associated with METH (Cadet and Krasnova, 2009; Goncalves et al., 2008; Hebert and O'Callaghan, 2000; Kelly et al., 2012; Ladenheim et al., 2000; Nakajima et al., 2004).

4. Death pathway activation: METH has been shown to activate both caspase-dependent and caspase-independent death cascades involving the both the ER and mitochondria (Cadet et al., 2007; Deng et al., 2001; Imam et al., 2001; Jayanthi et al., 2001; Jayanthi et al., 2005; Jayanthi et al., 2004). Alterations in proteins related to mitochondrial death pathways as a result of METH have been shown, including reductions in $\mathrm{Bcl}-2$ (an antiapoptotic protein), and increases in BAX and BID (inducers of apoptosis) (Cadet and Krasnova, 2009). Additionally, METH results in increases in Fas/FasL cell death signaling through caspase-3, an effect implicated in the toxic actions of the drug in striatal neurons (Jayanthi et al., 2005).

5. ER stress: ER stress elicited by METH has been reported in brain regions affected by the neurotoxic actions of the drug (Jayanthi et al., 2009). METH treatment results in the activation of 
pathways involved in ER stress induction and ER stress-induced cell death and these effects have been reported to be dependent on dopaminergic signaling (Beauvais et al., 2011; Jayanthi et al., 2004; Jayanthi et al., 2009).

6. Neuroinflammation: Neuroinflammation, resulting from the activation of glial cells, is hypothesized to be involved in the ability of METH to cause neurotoxicity (Cadet and Krasnova, 2009). Pro-inflammatory cytokines released from activated microglial cells as a result of METH are believed to be contributors to the drug's effects on dopaminergic nerve terminals within the striatum (Kelly et al., 2012). Specifically, a lack of Interleukin-6 (IL-6) has been shown to be protective against METH-induced neurotoxicity (Ladenheim et al., 2000).

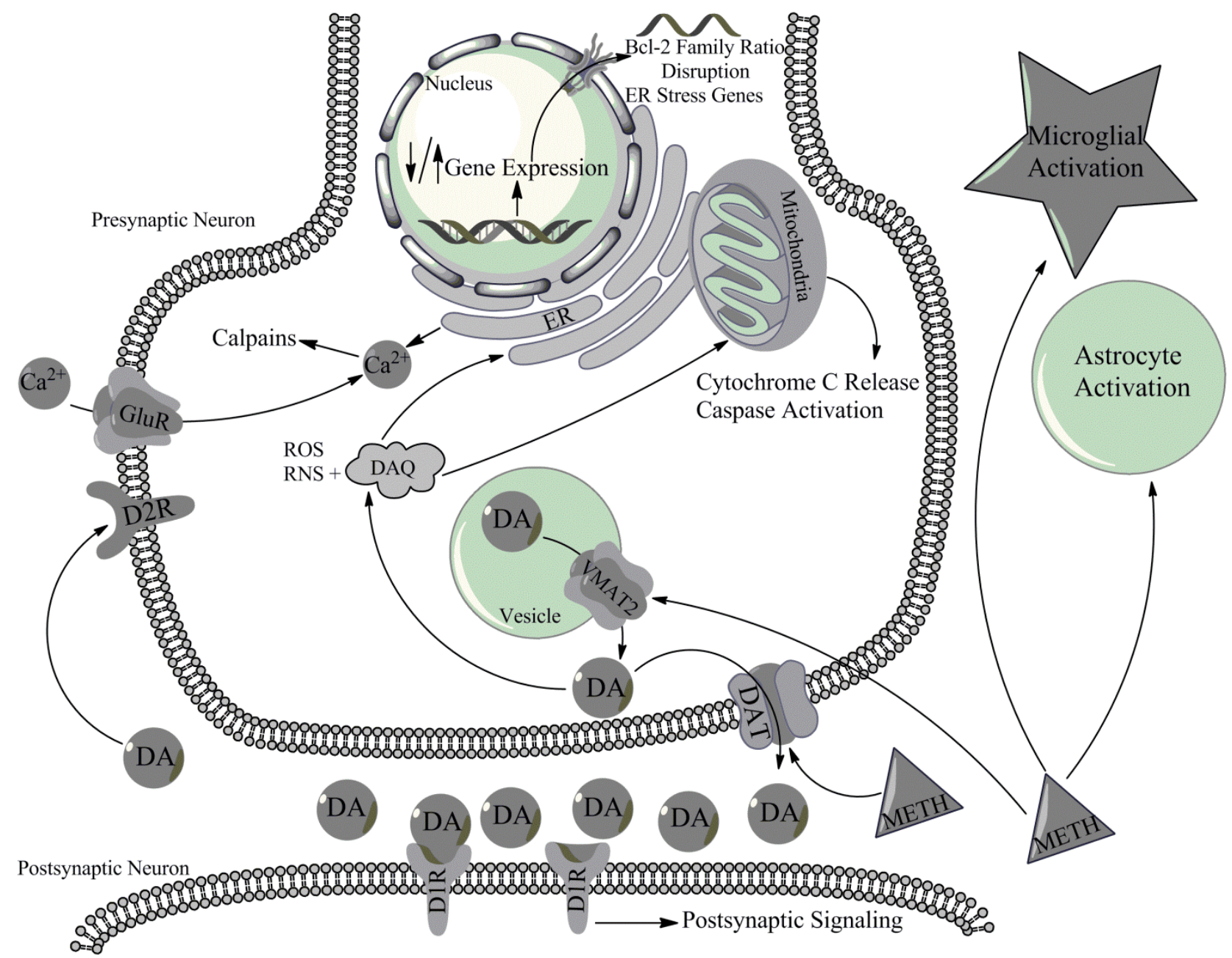


Figure 3.1. Proposed mechanisms of METH-induced dopaminergic neurotoxicity. METH is believed to result in toxicity through a variety of cellular mechanisms. These include excess dopamine (DA) converting to dopamine quinones (DAQ) and reactive dopamine species, gene expression alterations related to cellular toxicity, glutamatergic excitotoxicity, caspase cleavage and apoptosis signaling, ER stress induction, glial cell activation and subsequent release of pro-inflammatory cytokines from activated microglia.

\subsection{Sigma Receptors and METH}

One potentially promising target for the production of therapies aimed at counteracting the effects of METH, including neurotoxicity are sigma receptors. Evidence that sigma receptors play a role in the actions of METH began to emerge nearly 20 years ago (Ujike et al., 1992b). METH interacts directly with both subtypes of sigma receptors and is known to display a 22 -fold preference for sigma-1 receptors over sigma- 2 receptors $\left(K_{i} 2.16 \pm 0.25 \mu \mathrm{M}\right.$ and $46.67 \pm 10.34 \mu \mathrm{M}$ for sigma- 1 and sigma- 2 receptors, respectively) (Nguyen et al., 2005). Nguyen et al. also showed that interactions between METH and sigma-1 receptors are competitive in nature by using the selective sigma-1 receptor ligand $\left[{ }^{3} \mathrm{H}\right](+)$-pentazocine in saturation binding experiments conducted in rat brain homogenates (Nguyen et al., 2005). These studies, showing a direct interaction between METH and sigma-1 receptors, raised the possibility that METH exerts effects through direct interactions with sigma-1 receptors.

In addition to evidence for competitive interactions, accumulated data over the years suggests that the interaction of METH with sigma-1 receptors is complex. METH is believed to act as a sigma-1 receptor agonist and data supporting this comes from reports of the ability of sigma-1 receptor antagonists and antisense oligonucleotides to attenuate many effects of the drug (Matsumoto et al., 2008; Nguyen et al., 2005). However, the binding of classical sigma-1 receptor agonists to sigma-1 receptors results in the dissociation of the chaperone protein BiP and sigma-1 receptors at the MAM (Hayashi and Su, 2007b). METH produces effects that are atypical of classical sigma-1 receptor agonists in this assay (Hayashi and Su, 2007b), although the underlying reason for this difference is currently unknown. In addition, pharmacological dose response characterizations show that sigma-1 receptor antagonists lower the maximal effect of the dose response curve for METH in behavioral studies 
(Nguyen et al., 2005), a pattern that is indicative of non-competitive interactions at the receptor.

Together, the data suggest that although METH can interact competitively with the classical sigma-1 agonist binding site, its effects subsequent to binding are atypical of a classical agonist at sigma-1 receptors.

Therefore, it is noteworthy that sigma-1 receptors are involved in a variety of processes that may modulate responses to METH away from the binding site. First, sigma receptors modulate dopamine synthesis, release and uptake (Basianetto et al., 1995; Booth and Baldessarini, 1991; Gonzalez-Alvear and Werling, 1994; Weatherspoon et al., 1996; Weiser et al., 1995). Specifically, the sigma-1 receptor agonist (+)-pentazocine increases dopamine synthesis in rat striatum, and this effect is prevented with the sigma receptor antagonist 1-(4-fluorophenyl)-4-[4-(5-fluoropyrimidin-2-yl)piperazin1-yl]butan-1-ol (BMY-14802) (Booth and Baldessarini, 1991). Moreover, the activation of sigma-1 receptors results in increased dopaminergic neurotransmission in the striatum (Gonzalez-Alvear and Werling, 1994; Gonzalez and Werling, 1997). Another modality by which sigma-1 receptors may modulate responses to METH is through the alteration of cellular signaling. Sigma-1 receptors modulate a variety of signaling pathways including interactions with protein partners, modulation of ion channel function and alterations in signaling cascades relevant to neurotransmission (Su and Hayashi, 2003; Su et al., 2010a). Therefore, direct interactions with the sigma-1 receptor, altering dopaminergic neurotransmission and modulating signaling relevant to neurotransmission are three distinct modalities by which sigma-1 receptor ligands may affect the actions of METH.

The expression levels of sigma-1 receptors themselves are also altered after repeated METH administration. Increases in sigma-1 receptors, labeled with $\left[{ }^{3} \mathrm{H}\right](+)$-pentazocine, in the substantia nigra, cerebellum and the frontal cortex, result from repeated METH treatment (Itzhak, 1993). In addition, increases in sigma-1 receptor expression have been shown in response to self-administration of METH in rats (Hayashi et al., 2010; Stefanski et al., 2004). These results are believed to be indicative of sigma-1 
receptors playing a distinctive role in the learning and conditioning responses associated with METH self-administration (Hayashi et al., 2010).

Much less is known about the specific roles of sigma- 2 receptors in the actions of METH. This is primarily due to the current lack of molecular tools and truly selective ligands targeting sigma-2 receptors. It is hypothesized however that sigma-2 receptors are involved in many cellular responses relevant to the actions of METH, including neurotoxicity (Kaushal and Matsumoto, 2011). It is known that sigma- 2 receptor activation can result in apoptosis through the modulation of $\mathrm{Ca}^{2+}$ levels from both the ER and mitochondria (Cassano et al., 2009; Vilner and Bowen, 2000). Apoptosis elicited by sigma-2 activation can occur through caspase dependent and/or caspase independent processes (Bowen, 2000; Crawford and Bowen, 2002), both of which are implicated in the neurotoxic actions of METH (Cadet and Krasnova, 2009).

\subsection{Sigma Receptor Ligands as Potential Treatments for Negative Side Effects of METH}

Sigma receptor antagonists have been used in various animal models to mitigate many of the negative effects of METH. A synopsis of these results is included below and focuses on physiologic, behavioral and toxicity studies involving a variety of sigma receptor ligands and molecular techniques specifically targeting sigma-1 receptors.

\subsubsection{Locomotor activity studies}

METH treatment results in acute increases in locomotor stimulatory behavior in rodents and these effects are attenuated with pretreatment of the sigma receptor antagonists BD1047 and BD1063 (Nguyen et al., 2005). Evidence implicating an involvement of sigma-1 receptors in these effects arose from genetic knockdown studies. A 40\% reduction in sigma-1 receptor expression has been reported in the CNS of rodents using antisense oligonucleotides to block the translation of sigma-1 receptors 
(Matsumoto et al., 2001b), resulting in a marked decrease in METH-induced increases in locomotor activity, without affecting basal locomotor activity compared to naïve and mismatch controls (Nguyen et al., 2005). Contradictory to these results however, knockout of sigma-1 receptor expression does not ameliorate METH-induced hyperactivity (Fontanilla et al., 2009). Sigma-1 knockout mice in these studies displayed METH-induced increases in locomotor activity similar to METH treated wild type mice (Fontanilla et al., 2009). It is possible that in sigma-1 receptor knockout animals, sigma-2 receptors substitute some functionality for sigma-1 receptors and this is the rationale behind the discrepancy in results between the knockdown and knockout studies.

In contrast to the actions of antagonists, SA4503, a selective sigma-1 receptor agonist has varying dose-dependent effects on METH-induced locomotor activity, as it can both potentiate and attenuate METH-induced increases in locomotor activity (Rodvelt et al., 2011b). SA4503, at a dose of 1 $\mathrm{mg} / \mathrm{kg}$, potentiated METH-induced increases in locomotor activity, whereas 10 and $30 \mathrm{mg} / \mathrm{kg}$ pretreatments were shown to attenuate METH-induced hyperactivity. It should be noted, however, that $30 \mathrm{mg} / \mathrm{kg}$ also displayed sedative effects on its own (Rodvelt et al., 2011b). Other sigma-1 receptor agonists have been shown to not affect basal locomotor activity; therefore, the pattern of data suggests that SA4503 may be exerting effects through non-sigma-1 receptor sites at higher dosages (Okuyama et al., 1996; Xu and Domino, 1997). Combined, these results provide evidence that sigma-1 receptor ligands alter METH-induced locomotor activity, with antagonism generally providing protective effects.

\subsubsection{Drug discrimination paradigms}

Sigma receptor ligands have been shown to alter a variety of other METH-induced behaviors in addition to hyperactivity. SA4503 potentiates the ability of METH to substitute for cocaine in a drug discrimination paradigm in Sprague-Dawley rats (Rodvelt et al., 2011a). The potentiation by SA4503 resulted in a shift of the dose response curve to the left and an increase in the $\mathrm{ED}_{50}$ of $M E T H$ in this 
behavioral paradigm by nearly $200 \%$ (Rodvelt et al., 2011a). Interesting to note, is that in this same study, d-amphetamine, an amphetamine with low affinity for sigma-1 receptors, also substitutes for cocaine; however, these effects are unable to be potentiated with concomitant administration of SA4503 (Rodvelt et al., 2011a). These results are indicative of sigma-1 receptor agonism resulting in a potentiation of the METH discriminative stimulus response. These results appear to involve sigma-1 receptors as SA4503 was unable to potentiate the responses to an amphetamine that displays relatively little affinity for these receptors. These studies thus provide evidence that sigma-1 receptors are involved in the behavioral effects of METH beyond that of locomotor activity.

SA4503 has also been shown not to substitute for METH in a drug discrimination paradigm using rats trained to discriminate between saline and METH (Rodvelt et al., 2011b). However, when rats were pretreated with the sigma-1 receptor agonist SA4503 at a dose of $1 \mathrm{mg} / \mathrm{kg}$, there was a significant increase in the discriminatory effects of METH in these animals (Rodvelt et al., 2011b). This effect is not present when d-amphetamine was used in place of METH, providing further evidence that sigma-1 receptors are indeed responsible for this effect (Rodvelt et al., 2011b). SA4503 appears to display mixed effects in many behavioral paradigms relevant to the actions of METH. Although SA4503 potentiates METH discrimination in animals trained to discriminate between saline and cocaine and in animals trained to discriminate between saline and METH, it lacks the ability to substitute for METH on its own (Rodvelt et al., 2011b). It therefore appears that activation of sigma-1 receptors alone is not sufficient in and of itself to substitute for METH and it may be through a modulation of signaling that SA4503 potentiates these responses.

\subsubsection{Behavioral sensitization}

Mixed sigma-1/2 receptor ligands such as (R)-(+)-1-(4-chlorophenyl)-3-[4-(2-methoxyethyl) piperazin-1-yl]methyl-2-pyrrolidinone L-tartrate (MS-377), BMY 14802 and 3-(4-(4-cyclohexylpiperazin- 
1-yl)pentyl)-6-flourobenzo[d]thiazol-2(3H)-one (AZ66) have been shown to prevent behavioral sensitization induced by METH (Seminerio et al., 2012b; Takahashi et al., 2000; Ujike et al., 1992b). Behavioral sensitization is an in vivo experimental paradigm that uses locomotor activity as a quantifiable measure of neuroplastic changes in response to repeated treatments of a drug (Chen et al., 2009; Kalivas and Nakamura, 1999). These studies have provided evidence that sigma receptors are involved in the neurological changes associated with the sensitization to $\mathrm{METH}$, however the exact mechanisms by which this occurs is currently unclear.

\subsubsection{Hyperthermia}

In addition to mitigating METH-induced locomotor activity, sigma receptor antagonists have been shown to attenuate the acute physiologic effects of METH. Mixed sigma-1/2 receptor antagonists such as AC927, CM156 and SN79 attenuate the hyperthermia elicited by METH, at doses that do not alter basal body temperature on their own (Kaushal et al., 2011d; Kaushal et al., 2011f; Seminerio et al., 2011). Since METH-induced hyperthermia is one of the principal causes of death in METH overdose (Bowyer et al., 1994), therapies that can attenuate these potentially fatal increases in body temperature have high clinical significance.

\subsubsection{Neurotoxicity}

A variety of mixed sigma-1/sigma-2 antagonists have been shown to attenuate markers of METH-induced neurotoxicity in animal models. Pretreatment with mixed sigma- $1 / 2$ receptor antagonists such as AC927, CM156, AZ66 and SN79 mitigate decreases in striatal dopamine and/or serotonin levels, as well as reductions in striatal DAT and/or SERT expression, resulting from a neurotoxic regimen of METH (Kaushal and Matsumoto, 2011; Kaushal et al., 2012b; Matsumoto et al., 2008; Robson et al., 2012; Seminerio et al., 2012a; Seminerio et al., 2011). Prior to these studies, BMY 14802, a sigma-1/2 
receptor antagonist, was shown to attenuate METH-induced dopaminergic neurotoxicity; the effects of BMY14802 were initially attributed to its interactions with the dopaminergic system, and it was not until the aforementioned data using more selective compounds that the protective effects could be attributed to sigma receptors (Terleckyj and Sonsalla, 1994).

\begin{tabular}{|c|c|}
\hline Sigma Receptor Ligand Chemical Structure & Chemical Name \\
\hline & $\begin{array}{l}\text { AC927 } \\
\text { 1-(2-phenethyl)piperidine oxalate }\end{array}$ \\
\hline & $\begin{array}{l}\text { CM156 } \\
\text { 3-(4-(4-cyclohexylpiperazin-1- } \\
\text { yl)butyl)benzo[d]thiazole-2(3H)-thione }\end{array}$ \\
\hline & $\begin{array}{l}\text { AZ66 } \\
\text { 3-(4-(4-cyclohexylpiperazin-1-yl)pentyl)-6- } \\
\text { fluorobenzo[d]thiazol-2(3H)-one }\end{array}$ \\
\hline & $\begin{array}{l}\text { SN79 } \\
\text { 6-acetyl-3-(4-(4-(4-fluorophenyl)piperazin- } \\
\text { 1-yl)butyl)benzo[d]oxazol-2(3H)-one }\end{array}$ \\
\hline
\end{tabular}

Table 3.1. Chemical structures of sigma receptor ligands known to mitigate the neurotoxic effects of METH 
Currently, it is unclear what molecular effects are responsible for the ability of sigma receptor antagonists to mitigate the neurotoxic actions of METH. Many of the cellular effects of sigma receptors remain a mystery and little work has currently been conducted aimed at determining the molecular mechanisms by which sigma receptor antagonists mitigate many of the effects of METH. The purpose of the studies included in the remainder of this dissertation are aimed at beginning to delineate the mechanisms by which sigma receptor antagonists convey neuroprotective effects against METH using a combination of in vivo and in vitro models. The three specific aims of this section are therefore:

1. Determine if the ability of sigma receptor antagonists to mitigate METH-induced dopaminergic neurotoxicity is correlated to their ability to mitigate METH-induced hyperthermia.

2. Determine if a protective sigma receptor antagonist modulates METH-induced PERKmediated ER stress in an in vitro model.

3. Determine if a protective sigma receptor antagonist mitigates METH-induced neuroinflammation and glial cell activation in vivo. 
Chapter 4

Sigma receptor antagonist attenuation of METH-induced neurotoxicity is correlated to body temperature modulation 


\subsection{Introduction}

METH is an addictive psychostimulant whose use results in acute elevations in core body temperature (Krasnova and Cadet, 2009). Chronic usage of the drug has been shown to cause neurotoxic effects in specific regions of the brain, most notably the striatum in human subjects and animal models (Bowyer et al., 1994; Kaushal et al., 2011f; Volkow et al., 2001a). Moreover, METH has recently been connected to an increased risk of developing Parkinson's disease in chronic users (Callaghan et al., 2012). METH is currently listed as the second most abused illicit substance worldwide and there are no FDA approved pharmacotherapies for treating METH addiction or its other negative consequences (Rawson and Condon, 2007).

METH is believed to exert its effects through interactions with monoamine transporters and vesicular monoamine transporters at nerve terminals (Krasnova and Cadet, 2009). However, recent studies suggest METH may produce some of its effects through sigma receptors as pretreatment with sigma receptor antagonists attenuates METH-induced hyperthermia and provides neuroprotective effects in animal models of METH-induced neurotoxicity (Kaushal et al., 2011f; Matsumoto et al., 2008; Seminerio et al., 2011). METH interacts with both subtypes of sigma receptors, denoted sigma-1 and sigma-2, at physiologically relevant concentrations (Nguyen et al., 2005). In addition, sigma receptor ligands have been shown to modulate dopamine release and signaling pathways relevant to the behavioral and physiologic actions of METH (Gonzalez-Alvear and Werling, 1994; Liu et al., 2001).

It is believed that METH-induced hyperthermia is a contributing factor to METH-induced neurotoxicity (Kiyatkin and Sharma, 2009). There are several agents, however, that have been shown in rodent models to ameliorate the neurotoxic effects of METH without a concomitant attenuation of hyperthermia (Chipana et al., 2008; Escubedo et al., 2009; Tsuji et al., 2009). It remains unknown whether sigma receptor antagonists attenuate METH-induced neurotoxicity without mitigating METHinduced increases in core body temperature. In two distinct batches of animals, pretreatment with two 
separate sigma receptor antagonists failed to attenuate METH-induced hyperthermia. These results represent deviations from the normal pattern of data previously reported (Kaushal et al., 2011f; Matsumoto et al., 2008; Seminerio et al., 2011). Although the specific cause of this deviation remains unknown, it afforded the opportunity to determine the relationship between the ability of sigma receptor antagonists to attenuate $\mathrm{METH}$-induced hyperthermia and their ability to attenuate METHinduced dopaminergic neurotoxicity. The purpose of the current study was therefore to determine if sigma receptor antagonists are able to mitigate METH-induced dopaminergic neurotoxicity without a concomitant attenuation of METH-induced hyperthermia.

\subsection{Materials and Methods}

\subsubsection{Drugs and reagents}

Methamphetamine was purchased from Sigma-Aldrich (St. Louis, MO). N-phenethylpiperidine oxalate (AC927) was synthesized by converting the free base N-phenethylpiperidine (Sigma-Aldrich, Inc., St. Louis, MO) to the oxalate salt (Maeda et al., 2002). 3-(4-(4-Cyclohexylpiperazin-1-yl)pentyl)-6flourobenzo[d]thiazol-2(3H)-one (AZ66) was synthesized as previously described (Seminerio et al., 2012b). Dopamine Research ElA kits were purchased from Rocky Mountain Diagnostics (Colorado Springs, CO).

\subsubsection{Animals}

Male, Swiss Webster mice (24-28 g, Harlan, Indianapolis, IN; Frederick, MD) were used for all experiments. Animals were housed in groups of five with a 12:12-h light/dark cycle and food/water ad libitum. All experiments were performed as approved by the Institutional Animal Care and Use Committee at the West Virginia University Health Sciences Center. 


\subsubsection{Treatment procedure}

Mice underwent a repeated dosing experimental paradigm that has previously been shown to cause significant dopaminergic neurotoxicity in response to METH in vivo (Kaushal et al., 2011f). All treatments and procedures were conducted in the same way. Mice were transported from the animal housing facility to the testing area (laboratory) where they were acclimated for at least $1 \mathrm{~h}$ prior to initiation of experiments. Additionally, all experimental procedures were conducted between the hours of 9 AM and 6 PM. The doses for METH $(5 \mathrm{mg} / \mathrm{kg}$, i.p.) and the sigma receptor antagonists $(10 \mathrm{mg} / \mathrm{kg}$, i.p.) were selected based on earlier dose response characterizations (Matsumoto et al., 2008). Mice were randomly assigned to one of the following experimental groups: Saline+ Saline; Saline + METH; AC927 + Saline; AC927 + METH or AZ66 + Saline; AZ66 + METH. Saline or sigma receptor antagonist pretreatments occurred 15 min prior to treatments with either saline or METH. Mice received their designated treatment combination a total of four times, once every two hours. Core body temperature was recorded one hour after each treatment combination. Recordings were made with a Thermalert THS monitor (Physitemp Instruments Inc., Clifton, NJ). Mice were held at the base of the tail and a probe (RET-3) inserted approximately $2.5 \mathrm{~cm}$ past the rectum into the colon for $8-10 \mathrm{~s}$ until a rectal temperature was maintained for $3-4 \mathrm{~s}$. All experiments were conducted at room temperature $\left(20^{\circ} \mathrm{C}\right)$.

\subsubsection{Striatal dopamine measurements}

Dopamine ELISA kits (Rocky Mountain Diagnostics, Colorado Springs, CO) were used to quantify dopamine in the striatum. Briefly, animals were decapitated one week after undergoing treatment. Bilateral striatum samples were dissected and immediately flash frozen in liquid nitrogen and stored at $80^{\circ} \mathrm{C}$. Dopamine was measured according to manufacturer's protocols as previously reported (Kaushal et al., 2011f; Matsumoto et al., 2008). METH-induced reductions in striatal dopamine content were used as 
a marker of METH-induced dopaminergic neurotoxicity in the current study because it correlates well with other established markers, such as reductions in striatal dopamine transporter expression and tyrosine hydroxylase activity (Albers and Sonsalla, 1995; Kaushal et al., 2011f).

\subsubsection{Data analysis}

Body temperature recordings were analyzed using a two-way repeated measures ANOVA followed by post-hoc Bonferroni's analyses. Striatal dopamine measurements were analyzed using a one-way ANOVA, followed by post-hoc Tukey's multiple comparison's tests. A correlation of average body temperatures during the course of the experiment to striatal dopamine content was conducted using Pearson's correlation analysis. All analyses were conducted using GraphPad Prism 5.0 (San Diego, CA). $P<0.05$ was considered significant for all statistical analyses.

\subsection{Results}

A total of four distinct experiments were conducted: two utilizing pretreatments of either saline or AC927 (experiments $1 \mathrm{~A}$ and $1 \mathrm{~B}$ ), and two which utilized pretreatments of either saline or AZ66 (experiments $2 \mathrm{~A}$ and $2 \mathrm{~B}$ ). In experiment $1 \mathrm{~A}$ using AC927, two-way repeated measures ANOVA revealed significant differences in core body temperatures at different time points between groups $(p<0.01)$. Post-hoc Bonferroni's analysis revealed that METH treatment resulted in an increase in core body temperature compared to saline-treated animals at time points 2,3 and $4(t=4.22, p<0.001 ; t=6.13, p$ $<0.001 ; \mathrm{t}=4.95, \mathrm{p}<0.001$, respectively). Pretreatment with AC927 in experiment $1 \mathrm{~A}$ resulted in the mitigation of METH-induced hyperthermia at time points 2,3 and $4(t=3.49, p<0.01 ; t=6.02, p<$ $0.001 ; \mathrm{t}=4.61, \mathrm{p}<0.001$, respectively) (Figure 4.1.A.). 
A.

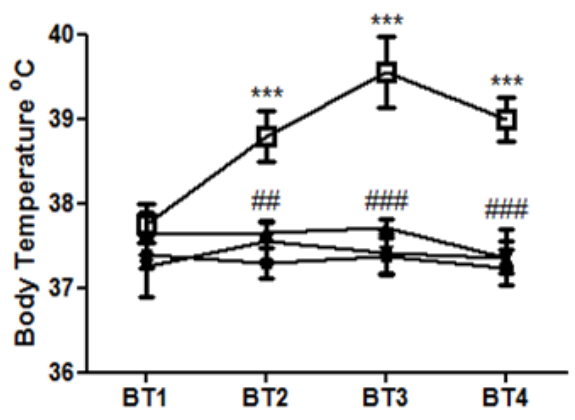

C.

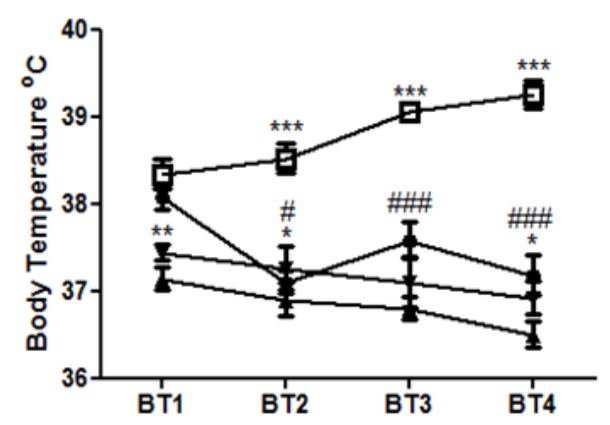

B.

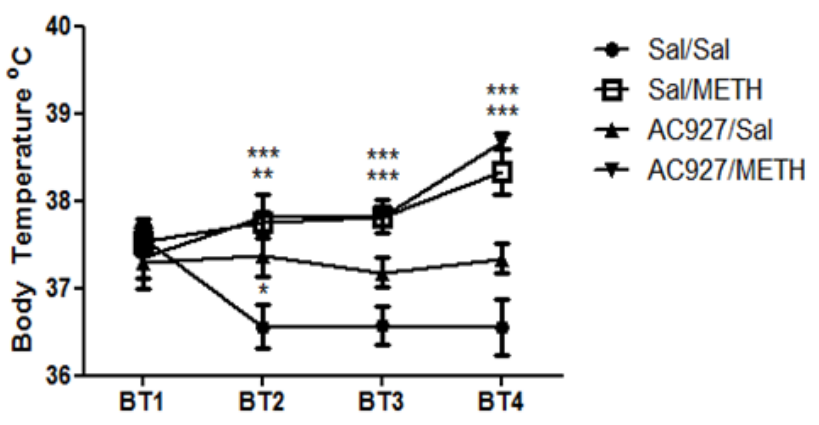

D.

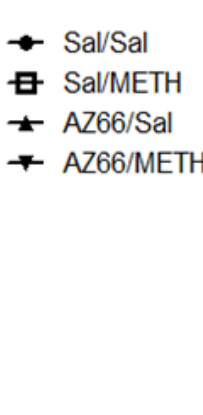

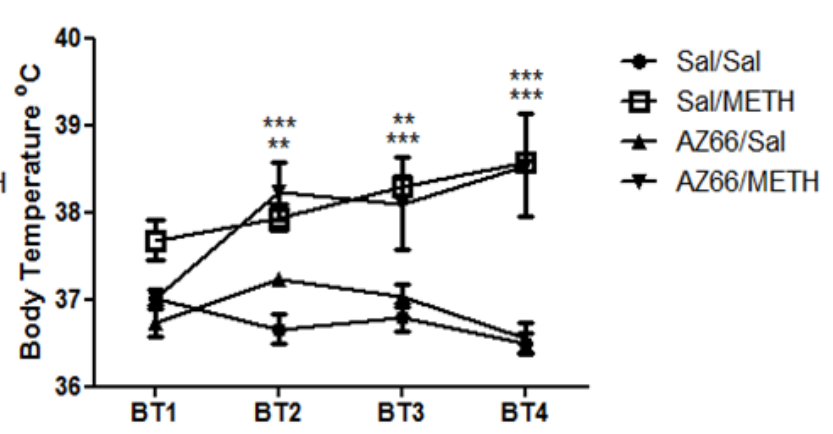

Figure 4.1. Male, Swiss Webster mice were treated with saline (Sal), AC927 (10 mg/kg, i.p.) or AZ66 (10 $\mathrm{mg} / \mathrm{kg}$, i.p.) followed $15 \mathrm{~min}$ later with either saline or methamphetamine (METH, $5 \mathrm{mg} / \mathrm{kg}$, i.p.). Each mouse received their designated treatment combination at two hr intervals a total of four times. Body temperature (BT) was measured one hour after each of the four treatments. (A) METH treatment results in a significant increase in core body temperature, attenuated by pretreatment with the sigma receptor antagonist AC927 (experiment 1A). ${ }^{* * *} p<0.001 \mathrm{vs.} \mathrm{Sal/Sal;} \mathrm{\# \#} p<0.01$, \#\#\# $p<0.0001 \mathrm{vs.} \mathrm{Sal/METH.}$ (B) In a secondary experiment (experiment 1B) pretreatment with AC927 failed to attenuate METHinduced hyperthermia at any timepoint. ${ }^{* *} \mathrm{p}<0.01,{ }^{* * *} \mathrm{p}<0.001 \mathrm{vs}$. Sal/Sal. (C) AZ66 pretreatment mitigates METH-induced hyperthermia (experiment 2A). ${ }^{*} p<0.05,{ }^{* *} p<0.01,{ }^{* * *} p<0.001 \mathrm{vs}$. Sal/Sal; $\# p<0.05$, \#\# p < 0.01, \#\#\# p < 0.001 vs. Sal/METH. (D) AZ66 failed to mitigate METH-induced core body temperature increases in a secondary experiment (experiment $2 \mathrm{~B}$ ). ${ }^{* *} \mathrm{p}<0.01,{ }^{* * *} \mathrm{p}<0.001 \mathrm{vs}$. Sal/Sal.

In experiment $1 \mathrm{~B}$, it was once again found that METH treatment alone resulted in a significant increase in core body temperature as compared to animals receiving saline at time points 2,3 and $4(t=$ 3.87, $\mathrm{p}<0.01 ; \mathrm{t}=4.00, \mathrm{p}<0.001 ; \mathrm{t}=5.74, \mathrm{p}<0.001$, respectively). Pretreatment in experiment $1 \mathrm{~B}$ with AC927, contrary to experiment $1 \mathrm{~A}$, failed to block METH-induced hyperthermia at any time point tested (n.s.) (Figure 4.1.B.). One-way ANOVA revealed differences between groups in striatal dopamine content in AC927 experiments $1 \mathrm{~A}$ and $1 \mathrm{~B}(\mathrm{~F}(4,35)=14.02, \mathrm{p}<0.0001)$. Post-hoc analyses with Tukey's multiple 
comparison tests revealed that METH treatment resulted in a significant reduction in striatal dopamine content as compared to saline $(q=8.87, p<0.001)$. Pretreatment with AC927 that mitigated the hyperthermic effects of METH was found to attenuate METH-induced striatal dopamine reductions $(\mathrm{q}=$ $6.64, \mathrm{p}<0.001)$. AC927 pretreatment that failed to attenuate METH-induced hyperthermia was unable to mitigate $\mathrm{METH}$-induced striatal dopamine reductions $(\mathrm{q}=2.43$, n.s.) (Figure 4.2.A.). Further analysis of these effects was conducted using a Pearson's correlation of average core body temperature over the course of the experiments and striatal dopamine content. It was found that the average body temperature of animals was correlated to striatal dopamine content one week post-treatment $(r=-0.66$, $p<0.0001, r^{2}=0.43$ ) (Figure 4.3.A.).

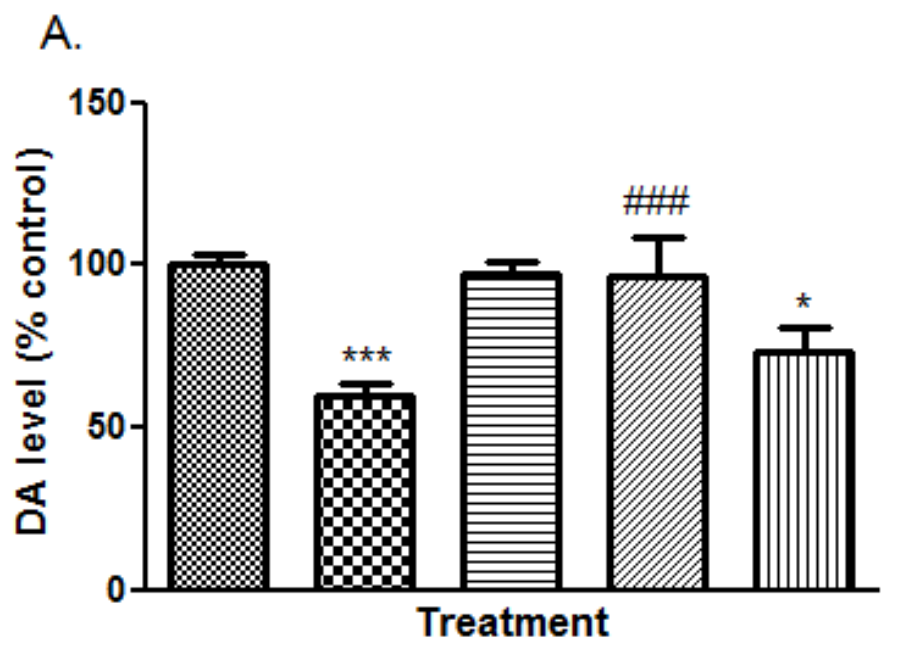

$\approx \mathrm{Sal} / \mathrm{Sal}$

Wal/METH

曰 AC927/Sal

ICI AC927/METH Exp. 1

而 AC927/METH Exp. 2

\section{B.}

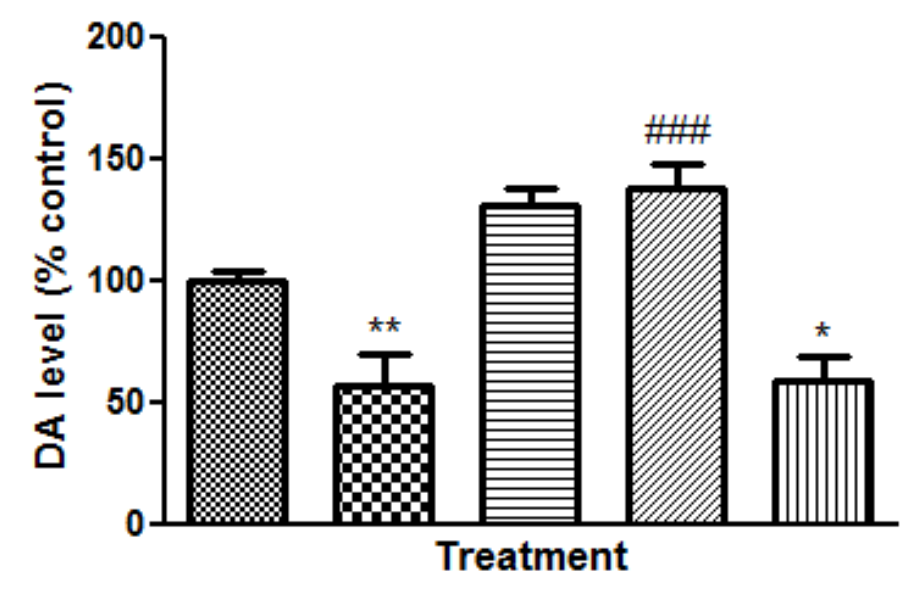

Sal/Sal

$\infty \mathrm{Sal} / \mathrm{METH}$

曰 AZ66/Sal

IZZ66/METH Exp. 1

而 AZ66/METH Exp. 2 
Figure 4.2. Male, Swiss Webster mice were treated with saline (Sal), AC927 (10 mg/kg, i.p.) or AZ66 (10 $\mathrm{mg} / \mathrm{kg}$, i.p.) followed $15 \mathrm{~min}$ later with either saline or methamphetamine (METH, $5 \mathrm{mg} / \mathrm{kg}$, i.p.). Each mouse received their designated treatment combination at two hr intervals a total of four times. Striatal tissues were collected from the mice one week post-treatment and dopamine (DA) levels measured. (A) AC927 pretreatment that blocks METH-induced hyperthermia (experiment $1 \mathrm{~A}$ ) attenuates METHinduced dopaminergic toxicity. AC927 that fails to block METH-induced hyperthermia (experiment 1B) also fails to mitigate dopaminergic toxicity elicited by METH. ${ }^{*} p<0.05, * * * p<0.001 \mathrm{vs}$. Sal/Sal; \#\#\# $<$ $0.001 \mathrm{vs.} \mathrm{Sal/METH.} \mathrm{(B)} \mathrm{Similar} \mathrm{to} \mathrm{pretreatment} \mathrm{with} \mathrm{AC927,} \mathrm{AZ66} \mathrm{pretreatment} \mathrm{that} \mathrm{mitigates} \mathrm{the}$ hyperthermic effects of METH also blocks METH-induced dopaminergic deficits (experiment 2A). Failure to block METH-induced hyperthermia with AZ66 results in a failure to attenuate METH-induced

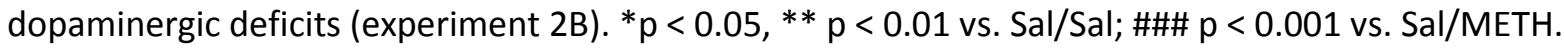

Two separate experiments utilizing the sigma receptor antagonist AZ66 were also conducted.

Two-way repeated measures ANOVA revealed differences in core body temperature between groups at different time points in experiment 2A utilizing AZ66 $(p<0.0001)$. Bonferroni's post-hoc analysis revealed that METH treatment significantly increased core body temperature, compared to saline treatment at time points 2,3 and 4 in experiment $2 A(t=5.62, p<0.001 ; t=5.85, p<0.001 ; t=8.23, p<$ 0.001, respectively). Pretreatment with AZ66 mitigated METH-induced hyperthermia at all three time points $(t=4.98, p<0.001 ; t=7.75, p<0.001 ; t=9.25, p<0.001 ;$ for time points 2,3 and 4 , respectively). Additionally, time point 1 significantly differed between these two groups $(t=3.56, p<$ 0.01) (Figure 4.1.C.).

In a second experiment involving AZ66 (experiment 2B), two-way repeated measures ANOVA revealed significant differences between groups and different time points $(p<0.0001)$. Similar to experiment $2 \mathrm{~A}, \mathrm{METH}$ treatment resulted in a significant increase in core body temperature as compared to saline treatment at time points 2,3 and $4(t=3.66, p<0.01 ; t=4.29, p<0.001 ; t=5.95, p$ $<0.001$; respectively). Unlike experiment 2A however, AZ66 pretreatment was found to not attenuate METH-induced hyperthermia at any time point tested (n.s.) (Figure 4.1.D.).

One-way ANOVA revealed significant differences between AZ66 experimental groups $2 \mathrm{~A}$ and 2B in striatal dopamine content $(F(4,35)=16.46, \mathrm{p}<0.0001)$. Post-hoc Tukey's analysis revealed that METH treatment resulted in a significant reduction in striatal dopamine content as compared to saline treated 
animals $(q=5.28, p<0.01$ ). In experiment $2 A$ in which AZ66 mitigated METH-induced hyperthermia, it also significantly attenuated the METH-induced reduction in striatal dopamine content $(q=8.12, p<$ 0.001). However, in experiment $2 B$, in which AZ66 pretreatment failed to mitigate METH-induced hyperthermia, it also failed to block METH-induced reductions in striatal dopamine content (n.s.) (Figure 2B). A Pearson's correlation revealed that the average core body temperature of animals in these two experiments was significantly correlated to striatal dopamine content measured one week post treatment $\left(r=-0.52, p<0.001, r^{2}=0.27\right)$ (Figure 4.3.B.).
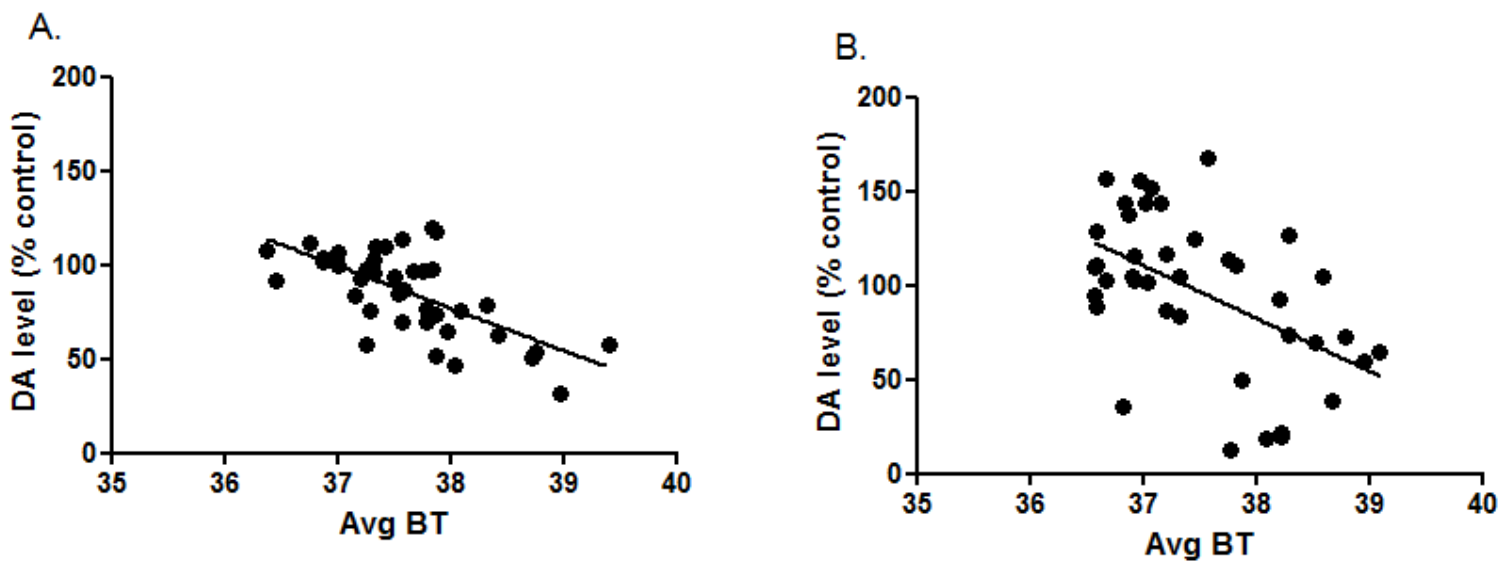

Figure 4.3. The ability of sigma receptor antagonists to attenuate METH-induced dopaminergic toxicity is correlated to core body temperature. (A) Average body temperatures (Avg BT) of animals during experiments utilizing AC927 (experiments $1 A$ and 1B) as compared to striatal dopamine (DA) levels ( $p<$ 0.0001). (B) Average body temperatures (Avg BT) of animals during experiments utilizing AZ66 (experiments $2 A$ and $2 B$ ) as compared to striatal dopamine (DA) content $(p<0.001)$.

\subsection{Discussion}

This study confirms how large a role core body temperature modulation plays in the ability of sigma receptor antagonists to mitigate METH-induced neurotoxicity. Our data unequivocally demonstrates that the blockade of $\mathrm{METH}$-induced hyperthermia by sigma receptor antagonists is correlated with their ability to attenuate METH-induced dopaminergic neurotoxicity. Moreover, this report shows that failure of a sigma receptor antagonist to attenuate $\mathrm{METH}$-induced hyperthermia also fails to mitigate the dopaminergic deficit elicited by METH in vivo. 
The relationship between METH-induced hyperthermia and neurotoxicity has been studied extensively in rodent models (Bowyer et al., 1994; Bowyer et al., 1992). High ambient temperatures have been shown to exacerbate the neurotoxic effects of METH, and reducing ambient temperatures has been shown to reduce the neurotoxic effects of the drug (Bowyer et al., 1994). The precise mechanisms by which this occurs are currently unknown and the protective effects do not appear to be specifically driven by core body temperature modulation (Albers and Sonsalla, 1995; Bowyer et al., 1992). Pharmacological reduction of core body temperature with resperine, for example, has been shown to not mitigate the neurotoxic effects of METH (Albers and Sonsalla, 1995). Additionally, some pharmacologic tools which fail to attenuate METH-induced hyperthermia have been shown to mitigate the effects of METH on markers of neurotoxicity (Chipana et al., 2008; Tsuji et al., 2009).

Mechanisms by which METH is thought to cause hyperthermia include increased reactive oxygen and nitrogen species (ROS/RNS) production, dopamine quinone production, and increased release of proinflammatory cytokines that have pyrogenic activity (Krasnova and Cadet, 2009). Interestingly, AC927 has been shown to attenuate METH-induced ROS/RNS generation, dopamine release and cellular toxicity in vitro (Kaushal et al., 2012a). The ability of selective sigma receptor antagonists such as AC927 to modulate dopamine release and therefore signaling events and possible reactive dopamine species formation may play a large role in their ability to modulate hyperthermic and neurotoxic responses to METH. The ability of selective sigma receptor antagonists such as AC927 and AZ66, to modulate these effects in vivo has yet to be determined; however it appears that sigma receptor ligands do have the ability to modulate many processes relevant to both the hyperthermic and neurotoxic effects of METH.

While the data supports the idea that in order for sigma receptor antagonists to mitigate dopaminergic deficits associated with METH, they must block the hyperthermic effects elicited by METH, further studies are needed to conclusively determine if the mitigation of METH-induced hyperthermia is 
truly a requirement for the blockade of dopaminergic neurotoxicity by sigma receptor antagonists or whether sigma receptor antagonists modulate common mediators that drive both hyperthermia and neurotoxicity. Experiments in which modulation of ambient temperature is used to study these effects have thus far been inconclusive because METH-induced lethality in the positive control group is a major limiting factor in rodent models (Bowyer et al., 1994; Seminerio et al., 2011).

In an alternative approach, an in vitro model of METH-induced neurotoxicity showed that when temperature was held constant, sigma receptor antagonists maintain their ability to attenuate the cytotoxicity caused by METH (Kaushal et al., 2011a; Kaushal et al., 2011b). Moreover, at higher in vitro temperatures, METH-induced cytotoxicity worsened, with sigma receptor antagonists still being able to attenuate these responses (Kaushal et al., 2011b). These in vitro studies demonstrate that sigma receptor antagonists are capable of mitigating METH-induced cytotoxicity at normal as well as elevated ambient temperatures in cell culture (Kaushal et al., 2011b), suggesting that the neuroprotective effects of the compounds are not dependent on temperature. This raises the possibility that under in vivo conditions, the apparent dependence of the ability of sigma receptor antagonists to mitigate METHinduced neurotoxicity may stem from sigma-mediated attenuation of mediators common to both hyperthermia and neurotoxicity.

It should be noted that the underlying cause of the differences in response between the responsive (experiments $1 \mathrm{~A}$ and $2 \mathrm{~A}$ ) and unresponsive (experiments $1 \mathrm{~B}$ and $2 \mathrm{~B}$ ) groups remain unknown, with the testing of the groups occurring weeks to months apart. Ambient temperature alterations have been shown to alter dopaminergic neurotoxicity in response to neurotoxic METH treatment regimens in rodents (Bowyer et al., 1992). Ambient temperature and other environmental variables, however, were accounted for in the above described experiments, and no unusual variations could be documented. Although all the animals were of the same outbred strain, from the same vendor, it is possible that an unidentified genetic variation may have existed between the batches of mice. 
Although nothing is known about variations in sigma-2 receptors, splice variants and polymorphisms in sigma-1 receptors have been reported, with some ramifications for function (Shioda et al., 2012). However, such variations would be expected to be distributed across different batches of mice, rather than affecting entire batches as was observed herein.

Due to the serendipitous nature of this study, only one measure of dopaminergic neurotoxicity was used in the current report. However, it should be emphasized that numerous studies have confirmed concomitant changes in other markers of dopaminergic neurotoxicity along with changes in striatal dopamine levels under conditions similar to those used in the present study (Bowyer et al., 1994; Kaushal et al., 2011f). In addition, AC927 has previously been shown to attenuate METH-induced decreases in striatal dopamine content, dopamine transporters and striatal serotonin content and serotonin transporters in vivo (Matsumoto et al., 2008; Seminerio et al., 2011), suggesting that the alterations in striatal dopamine levels measured herein serve as a reasonable marker of METH-induced dopaminergic neurotoxicity. The determination as to whether similar trends exist with regards to METHinduced serotonergic neurotoxicity and other markers of central nervous system toxicity has yet to be made.

In conclusion, the ability of sigma receptor antagonists to mitigate METH-induced neurotoxicity is closely associated with their ability to attenuate the hyperthermic effects of METH in vivo. Additional studies are warranted to further define the exact role of hyperthermia in the neurotoxic actions of METH. Moreover, further investigations are needed to identify the mechanisms through which sigma receptor antagonists reduce the hyperthermic and neurotoxic effects of $\mathrm{METH}$, and to determine whether the ability of sigma receptor antagonists to mitigate METH-induced neurotoxicity is dependent on the modulation of body temperature. 


\subsection{Contributions}

Matthew Robson, Michael Seminerio, Ph.D. and Jamaluddin Shaikh, Ph.D. conducted in vivo body temperature experiments and striatal dopamine measurements.

This chapter has previously been published as:

Robson MJ, Seminerio MJ, McCurdy CR, Coop A, Matsumoto RR. Sigma receptor antagonist attenuation of methamphetamine-induced neurotoxicity is correlated to body temperature modulation. Pharmacological Reports. 2013. 65(2): 343-349 


\section{Chapter 5}

AC927 Pretreatment Mitigates METH-Induced Changes in Striatal Gene Expression 


\subsection{Introduction}

METH treatment is known to result in neurotoxicity in striatal regions of the brain, a side effect for which there are currently no FDA approved pharmaceutical treatments (Volkow et al., 2001a; Volkow et al., 2001b). METH is known to exert several of its effects through the modulation of sigma receptors and sigma receptor antagonists have been shown to mitigate the behavioral and physiological effects of METH administration in rodent models, including METH-induced neurotoxicity (Matsumoto et al., 2008; Nguyen et al., 2005; Seminerio et al., 2011). The molecular mechanisms by which METH results in neurotoxicity are currently not entirely elucidated and hypothesized mechanisms are discussed in previous chapters. Furthermore, the precise molecular mechanisms by which sigma receptor antagonists mitigate the neurotoxic actions of METH are unclear.

It is known that METH administration results in changes in gene expression related to cellular toxicity in the striatum of rodents administered neurotoxic regimens of the drug (Jayanthi et al., 2001; Jayanthi et al., 2009; Kuhn et al., 2006; Thomas et al., 2004b). The determination as to whether sigma receptors have a role in $\mathrm{METH}$-induced genetic alterations has yet to be made. Furthermore, the ability of sigma receptors to modulate gene expression related specifically to cellular toxicity associated with METH has yet to be studied. Therefore, the primary goal of the current study was to determine the genetic changes in the striatum that result from the administration of a METH regimen known to result in dopaminergic neurotoxicity, and which of these genetic alterations are modulated by pretreatment with a selective sigma receptor antagonist known to mitigate $\mathrm{METH}$-induced dopaminergic neurotoxicity.

\subsection{Materials and Methods}

\subsubsection{Drugs and Reagents}


Methamphetamine was purchased from Sigma-Aldrich (St. Louis, MO). N-phenethylpiperidine oxalate (AC927) was synthesized by converting the free base N-phenethylpiperidine (Sigma-Aldrich, Inc., St. Louis, MO) to the oxalate salt (Maeda et al., 2002)

\subsubsection{Animals}

Male, Swiss Webster mice (24-28 g, Harlan, Indianapolis, IN; Frederick, MD) were used for all experiments. Animals were housed in groups of five with a 12:12-h light/dark cycle and food/water ad libitum. All experiments were performed as approved by the Institutional Animal Care and Use Committee at the West Virginia University Health Sciences Center.

\subsubsection{Drug Treatments}

Mice underwent a repeated dosing experimental paradigm that has previously been shown to cause significant dopaminergic neurotoxicity in response to METH in vivo (Kaushal et al., 2011f). All treatments and procedures were conducted in the same way. Mice were transported from the animal housing facility to the testing area (laboratory) where they were acclimated for at least $1 \mathrm{~h}$ prior to initiation of experiments. Additionally, all experimental procedures were conducted between the hours of 9 AM and 6 PM. The doses for METH (5 mg/kg, i.p.) and AC927 (10 mg/kg, i.p.) were selected based on earlier dose response characterizations (Matsumoto et al., 2008). Mice were randomly distributed for each experiment into one of four treatment groups: 1) Saline/Saline (0.1 ml/10 g body weight), 2) Saline/METH 5 mg/kg, 3) AC927 10 mg/kg/Saline, or 4) AC927 10 mg/kg/METH 5 mg/kg. The first compound listed in each pair (Saline or AC927) was administered intraperitoneally (i.p.) as a pretreatment 15 min prior to the second compound in each treatment group (Saline or METH, i.p.). Each animal underwent four pretreatments/treatments at $2 \mathrm{~h}$ intervals as previously described (Kaushal et al., 2012b). Animals were sacrificed $6 \mathrm{~h}$ post-treatment (time after last injection), brains removed and 
bilateral striatum samples excised, flash frozen using liquid nitrogen and then stored at $-80^{\circ} \mathrm{C}$ for future use.

\subsubsection{RNA Isolation and Microarray}

Total RNA was then extracted from flash frozen striatum samples using Trizol reagent (Invitrogen, Grand Island, NY) according to manufacturer's instructions. The total RNA concentration for each sample was quantified by spectral absorption, and the purity of the sample checked to confirm that the $260 / 280$ ratio was in the range of 1.8-2.1. Additionally, RNA was quantified using a Nanodrop (Fisher Scientific, Pittsburgh, PA). The RNA quality was checked on a Bioanalyzer (Agilent, Santa Clara, CA). Two hundred fifty ng of each RNA sample was processed using the Ambion WT Expression Kit (Grand Island, NY) according to the manufacturer's instructions. The typical yield from a WT Expression Kit reaction was 6-9 $\mu \mathrm{g}$ of cDNA. The required amount of cDNA $(5.5 \mu \mathrm{g})$ was processed for fragmentation and biotin labeling using the Gene Chip WT Terminal Labeling Kit (Affymetrix, Santa Clara, CA). The efficiency of each fragmentation reaction was checked via Agilent Bioanalyzer. The entire reaction of fragmented and biotin-labeled cDNA (50 $\mu$ l) with added hybridization controls was hybridized to the mouse GeneChip 1.0 ST Exon Arrays (Affymetrix) at $45^{\circ} \mathrm{C}$ for $17 \mathrm{~h}$ in a GeneChip Hybridization Oven 640 (Affymetrix). Mouse GeneChip 1.0 ST Exon Arrays were stained using the FS 450_0001 protocol in a GeneChip Fluidics Station 450 (Affymetrix). Briefly, biotin-labeled cDNA was reacted using two rounds of washes with a solution containing a streptavidin-phycoerythrin complex, with an intermediate treatment of biotin-labeled anti-streptadvidin antibody to amplify the signal. Phycoerythrin labeling was detected within the Affymetrix GeneChip Scanner 3000 7G plus using $532 \mathrm{~nm}$ light and detected by a photomultiplier tube. Expression Consol software (Affymetrix) was used to check quality controls of hybridized chips. 


\subsubsection{Data Analysis}

Microarray results were analyzed using ANOVA, in combination with either a fold change greater than 2 for all genes or a false discovery rate (FDR) correction. $p<0.05$ was considered statistically significant for all studies contained within this chapter.

\subsection{Results}

\subsection{1. $p<0.05$ and fold change greater than 2}

Striatal gene changes elicited by METH treatment that were blocked by AC927 pretreatment through first analysis method are listed below.

\begin{tabular}{|l|l|l|l|l|r|}
\hline Gene Symbol & Reference & $\begin{array}{l}\text { p-value(M } \\
\text { vs. Sal) }\end{array}$ & $\begin{array}{l}\text { Fold- } \\
\text { Change( } \\
\text { M vs. Sal) }\end{array}$ & $\begin{array}{l}\text { p-value(AM } \\
\text { vs. AC) }\end{array}$ & $\begin{array}{l}\text { Fold- } \\
\text { Change(AM } \\
\text { vs. AC) }\end{array}$ \\
\hline Lilrb4 & NM_013532 & 0.00180553 & 9.69286 & 0.0741054 & 2.76968 \\
\hline Gfap & NM_001131020 & 0.000949219 & 3.11686 & 0.0339675 & 1.7703 \\
\hline Ch25h & NM_009890 & 0.00626758 & 2.7104 & 0.11481 & 1.61622 \\
\hline Slc14a1 & NM_001171010 & 0.000116953 & 2.22542 & 0.00289043 & 1.62514 \\
& & & & & \\
\hline Hectd2 & NM_001163471 & $2.94 E-05$ & 2.14606 & 0.000123207 & 1.86743 \\
& & & & & \\
\hline Rasl11b & NM_026878 & 0.00141089 & 2.09226 & 0.0385694 & 1.4663 \\
& & & & & \\
\hline Pde1a & NM_001159582 & 0.0419207 & 2.08911 & & \\
\hline & & & & & \\
\hline
\end{tabular}




\begin{tabular}{|c|c|c|c|c|c|}
\hline Nudt10 & NM_001031664 & 0.000615864 & 2.06335 & 0.0439124 & 1.37452 \\
\hline $\mathrm{Cd} 44$ & NM_009851 & 6.81E-05 & 2.06329 & 0.00149471 & 1.57639 \\
\hline Id 2 & NM_010496 & 0.000844173 & 2.04379 & 0.0441542 & 1.38998 \\
\hline Olfr20 & NM_146923 & 0.0298164 & 2.0244 & 0.711642 & 1.10785 \\
\hline Stc1 & NM_009285 & 0.00103342 & -2.01013 & 0.0264272 & -1.45948 \\
\hline Olfr1480 & NM_207575 & 0.00267988 & -2.07149 & 0.35788 & -1.18046 \\
\hline Olfr1501 & NM_146633 & 0.0377525 & -2.14437 & 0.57827 & -1.19457 \\
\hline Ahcy & NM_016661 & 0.00735228 & -2.24096 & 0.365986 & 1.24225 \\
\hline Slc39a1 & NM_013901 & 0.0329088 & -2.31698 & 0.943762 & 1.02404 \\
\hline 5930434B04Rik & ENSMUST00000114005 & 0.00262656 & -2.37444 & 0.444594 & -1.17565 \\
\hline Fthl17 & NM_031261 & 0.02022 & -2.78129 & 0.573186 & 1.23113 \\
\hline Zfp617 & NM_133358 & 0.0353633 & -2.79772 & 0.896814 & -1.05599 \\
\hline Lce3b & NM_025501 & 0.0197789 & -3.02109 & 0.37619 & 1.4287 \\
\hline Zc3h3 & NM_172121 & 0.01694 & -3.06143 & 0.686689 & -1.16855 \\
\hline Ttr & NM_013697 & 0.0456178 & -4.04402 & 0.755256 & 1.21 \\
\hline
\end{tabular}

Table 5.1. List of genes obtained through ANOVA and fold change greater than 2 analysis. Genes contained within table were shown to be modulated by METH treatment and these effects were mitigated by pretreatment with the protective sigma receptor antagonist $A C 927$. Sal = Saline/Saline, $M=$ Sal/METH 5 mg/kg, AC = AC927 10 mg/kg/ Sal, AM = AC927 10 mg/kg/ METH 5 mg/kg. 
5.3.2. $p<0.05$ and false discovery rate (FDR) correction analysis

Genetic changes that were blocked by AC927 treatment when analyzed using ANOVA and FDR correction are listed below.

\begin{tabular}{|c|c|c|c|c|c|}
\hline Gene Symbol & $\begin{array}{l}\text { Reference } \\
\text { Sequence }\end{array}$ & $\begin{array}{l}\text { p-value (M } \\
\text { vs. Sal) }\end{array}$ & $\begin{array}{l}\text { Fold-Change } \\
\text { (M vs. Sal) }\end{array}$ & $\begin{array}{l}\text { p-value (AM } \\
\text { vs. AC) }\end{array}$ & $\begin{array}{l}\text { Fold-Change } \\
\text { (AM vs. AC) }\end{array}$ \\
\hline Osmr & NM_011019 & 0.0001927 & 4.13795 & 0.00159139 & 2.78749 \\
\hline Nptx1 & NM_008730 & 0.00035427 & 2.38875 & 0.000797363 & 2.15714 \\
\hline Slc14a1 & NM_001171010 & 0.00011695 & 2.22542 & 0.00289043 & 1.62514 \\
\hline $\mathrm{Cd} 44$ & NM_009851 & $6.81 \mathrm{E}-05$ & 2.06329 & 0.00149471 & 1.57639 \\
\hline Hbegf & NM_010415 & 0.00036405 & 1.99337 & 0.0006412 & 1.8827 \\
\hline Adamts1 & NM_009621 & 0.00035377 & 1.93685 & 0.000527136 & 1.8631 \\
\hline Mtmr11 & NM_181409 & 0.0003504 & 1.89723 & 0.000823128 & 1.75354 \\
\hline Acvr2a & NM_007396 & $5.50 \mathrm{E}-05$ & 1.88613 & 0.000268993 & 1.65707 \\
\hline Zfp131 & NM_028245 & $8.51 \mathrm{E}-06$ & 1.85103 & 0.000305029 & 1.45181 \\
\hline Plek & NM_019549 & 0.00026016 & 1.83277 & 0.00290717 & 1.51095 \\
\hline Msn & NM_010833 & 0.00033894 & 1.70161 & 0.014071 & 1.32184 \\
\hline Pdzrn3 & NM_018884 & 0.00013631 & 1.68695 & 0.00483103 & 1.34463 \\
\hline Hsph1 & NM_013559 & 0.00014295 & 1.6495 & 0.00640477 & 1.31097 \\
\hline Khdrbs3 & NM_010158 & $3.56 \mathrm{E}-05$ & 1.62341 & 0.000277688 & 1.43521 \\
\hline TIr13 & NM_205820 & 0.00030646 & 1.57293 & 0.000837112 & 1.4745 \\
\hline Tubb6 & NM_026473 & 0.0002387 & 1.56219 & 0.00030785 & 1.5366 \\
\hline Scg2 & NM_009129 & 0.00035852 & 1.53699 & 0.000758903 & 1.46687 \\
\hline Cdh11 & NM_009866 & 0.00024785 & 1.52405 & 0.000535637 & 1.45532 \\
\hline
\end{tabular}




\begin{tabular}{|c|c|c|c|c|c|}
\hline Slc35f5 & NM_028787 & 0.00034225 & 1.51268 & 0.701781 & 1.028 \\
\hline Cd68 & NM_009853 & 4.71E-05 & 1.5039 & 0.000457272 & 1.34113 \\
\hline Slc3a2 & NM_001161413 & $3.19 \mathrm{E}-05$ & 1.50293 & 0.00490767 & 1.20625 \\
\hline Rheb & NM_053075 & 0.00014106 & 1.49405 & 0.00693648 & 1.23805 \\
\hline C1galt1 & NM_052993 & 0.00023449 & 1.47929 & 0.00350786 & 1.28942 \\
\hline Ptgfrn & NM_011197 & 0.00021078 & 1.44497 & 0.00312876 & 1.27127 \\
\hline Irf8 & NM_008320 & 0.00036992 & 1.4294 & 0.00449511 & 1.26797 \\
\hline Aox1 & NM_009676 & 0.00034196 & 1.42651 & 0.00316922 & 1.28185 \\
\hline Ppm1l & NM_178726 & 0.00034436 & 1.3953 & 0.00211272 & 1.28392 \\
\hline $\mathrm{Npl}$ & NM_028749 & 0.00026462 & 1.38622 & 0.000305569 & 1.37667 \\
\hline Zdhhc2 & NM_178395 & 0.00016223 & 1.38348 & 0.0588858 & 1.11358 \\
\hline Tmem87a & NM_173734 & 0.00023935 & 1.37237 & 0.922154 & -1.0051 \\
\hline Atg2b & NM_029654 & 0.00026218 & 1.35215 & 0.00555849 & 1.20101 \\
\hline Grip1 & NM_028736 & 0.00015675 & 1.30055 & 0.000197567 & 1.28927 \\
\hline Rab3gap2 & NM_001163754 & 0.00035057 & 1.28556 & 0.160574 & 1.06773 \\
\hline Ptpn12 & NM_011203 & 8.80E-05 & 1.27151 & 0.000258994 & 1.22809 \\
\hline Stt3b & NM_024222 & $2.53 \mathrm{E}-05$ & 1.26962 & 0.00626664 & 1.10704 \\
\hline Pfkp & NM_019703 & 0.00018972 & 1.26258 & 0.0130746 & 1.12083 \\
\hline Pdia4 & NM_009787 & 0.00019396 & 1.26097 & 0.000795363 & 1.20601 \\
\hline Stt3a & NM_008408 & 0.00033717 & 1.22312 & 0.443214 & -1.02762 \\
\hline Eif3b & NM_133916 & 0.00013736 & 1.21472 & 0.00415003 & 1.12003 \\
\hline L3mbtl3 & NM_172787 & 5.93E-05 & 1.17696 & 0.0201281 & 1.0634 \\
\hline Mrps5 & NM_029963 & $1.47 \mathrm{E}-05$ & 1.09198 & 0.478552 & -1.00707 \\
\hline
\end{tabular}




\begin{tabular}{|c|c|c|c|c|c|}
\hline Dennd1a & NM_146122 & 0.00011191 & -1.12406 & 0.140571 & -1.02768 \\
\hline Spen & NM_019763 & 0.00013004 & -1.12638 & 0.292816 & 1.01973 \\
\hline Sf1 & NM_001110791 & 0.000196 & -1.12755 & 0.0128729 & 1.06099 \\
\hline Shank3 & NM_021423 & 0.00028295 & -1.13448 & 0.608111 & -1.01106 \\
\hline Ampd2 & NM_028779 & 0.00010417 & -1.14633 & 0.0215159 & -1.0565 \\
\hline Rhot2 & NM_145999 & 0.00013083 & -1.15318 & 0.367114 & -1.02008 \\
\hline Dlgap3 & NM_198618 & 0.00028162 & -1.15459 & 0.0147093 & 1.07542 \\
\hline Fads1 & NM_146094 & 0.00013968 & -1.15721 & 0.00293909 & -1.0949 \\
\hline Pqbp1 & NM_019478 & 0.00028344 & -1.16095 & 0.0053691 & -1.09664 \\
\hline Vegfb & NM_011697 & 0.00021857 & -1.16114 & 0.0655957 & -1.05137 \\
\hline Cabc1 & NM_023341 & 0.00012736 & -1.16304 & 0.000884201 & -1.11951 \\
\hline Mmab & NM_029956 & 0.0001297 & -1.16533 & 0.0034468 & -1.09572 \\
\hline Clptm1 & NM_019649 & 7.95E-05 & -1.16895 & 0.0111738 & -1.07211 \\
\hline Ranbp10 & NM_145824 & 0.00036617 & -1.18075 & 0.0267285 & 1.0794 \\
\hline Srrm2 & NM_175229 & $1.40 \mathrm{E}-05$ & -1.18108 & 0.023722 & -1.05082 \\
\hline Phf12 & NM_174852 & 3.07E-05 & -1.18255 & 0.014224 & -1.06428 \\
\hline Sphk2 & NM_203280 & 0.00020608 & -1.19402 & 0.211459 & 1.03826 \\
\hline Bcorl1 & NM_178782 & 0.00014795 & -1.19507 & 0.000321274 & -1.17235 \\
\hline Gnb2 & NM_010312 & 0.00023138 & -1.19538 & 0.200889 & -1.04024 \\
\hline Ptpn5 & NM_001163565 & 9.09E-05 & -1.19737 & 0.836896 & -1.00532 \\
\hline Eps15I1 & NM_007944 & 5.85E-05 & -1.19764 & 0.00597495 & -1.09097 \\
\hline Hexdc & NM_001001333 & 0.00013112 & -1.20473 & 0.000636658 & -1.15857 \\
\hline 1810031K17Rik & BC013714 & 0.0003549 & -1.20725 & 0.0120652 & -1.10827 \\
\hline
\end{tabular}




\begin{tabular}{|c|c|c|c|c|c|}
\hline Slc25a37 & NM_026331 & 0.00034177 & -1.2161 & 0.00641494 & -1.12782 \\
\hline Mink1 & NM_001045959 & 5.86E-05 & -1.2188 & 0.0245697 & -1.07379 \\
\hline Mapk4 & NM_172632 & 0.00035993 & -1.22172 & 0.0265024 & -1.09641 \\
\hline Med16 & NM_198107 & 0.00019771 & -1.22888 & 0.086139 & -1.06448 \\
\hline Grin1 & NM_001177656 & 3.37E-05 & -1.23253 & 0.00689277 & -1.09524 \\
\hline Sh3bgrl3 & NM_080559 & 0.00023163 & -1.24354 & 0.9106 & 1.00401 \\
\hline Lrch4 & NM_146164 & 0.00024427 & -1.24626 & 0.199928 & -1.0504 \\
\hline Mllt6 & NM_139311 & 0.00026901 & -1.25335 & 0.442613 & -1.03002 \\
\hline Smarcd1 & NM_031842 & $1.96 \mathrm{E}-05$ & -1.25533 & 0.000648623 & -1.14734 \\
\hline Haghl & NM_026897 & 0.00029796 & -1.26519 & 0.000254525 & -1.27226 \\
\hline Rin1 & NM_145495 & 5.43E-05 & -1.2705 & 0.15695 & -1.04936 \\
\hline Epha6 & NM_007938 & $1.42 \mathrm{E}-05$ & -1.27262 & 0.000795612 & -1.14455 \\
\hline Efnb2 & NM_010111 & 0.00029619 & -1.27495 & 0.0601893 & -1.09132 \\
\hline Lmo2 & NM_008505 & 0.00014984 & -1.28077 & 0.00505848 & -1.15127 \\
\hline Syt9 & NM_021889 & 2.37E-06 & -1.28587 & 0.000194487 & -1.14722 \\
\hline Icam5 & NM_008319 & $3.70 \mathrm{E}-05$ & -1.28657 & 0.00715915 & -1.1166 \\
\hline Zmynd10 & NM_053253 & 0.00022291 & -1.28681 & 0.0841946 & -1.08155 \\
\hline Axin2 & NM_015732 & 0.00027593 & -1.28709 & 0.000528035 & -1.25715 \\
\hline Stxbp2 & NM_011503 & 0.00018301 & -1.29275 & 0.00418683 & -1.16852 \\
\hline Slc22a8 & NM_031194 & 0.00015181 & -1.29613 & 0.0420327 & -1.09801 \\
\hline Raver2 & NM_183024 & 0.0001222 & -1.29839 & 0.00531884 & -1.15374 \\
\hline Shkbp1 & NM_138676 & 5.64E-05 & -1.30145 & 0.415263 & -1.02975 \\
\hline Phyhip & NM_145981 & 0.00019558 & -1.30592 & 0.0194023 & -1.12798 \\
\hline
\end{tabular}




\begin{tabular}{|c|c|c|c|c|c|}
\hline S1pr1 & NM_007901 & 9.75E-05 & -1.30978 & 0.000922063 & -1.2127 \\
\hline Сур2ј9 & NM_028979 & 0.0003406 & -1.31476 & 0.0143913 & -1.1538 \\
\hline Sez6 & NM_021286 & 8.85E-05 & -1.32525 & 0.0259069 & -1.11188 \\
\hline Hdac11 & NM_144919 & $1.73 E-05$ & -1.35094 & 0.00950187 & -1.1188 \\
\hline Reep6 & NM_139292 & 0.00035315 & -1.35192 & 0.0138459 & -1.17327 \\
\hline Rgs14 & NM_016758 & 0.00017402 & -1.35962 & 0.0114328 & -1.16485 \\
\hline Wasf1 & NM_031877 & $9.53 \mathrm{E}-06$ & -1.36059 & 0.00145494 & -1.15995 \\
\hline Gm996 & NM_001005424 & 0.00033003 & -1.36086 & 0.00256347 & -1.24889 \\
\hline Zdhhc12 & NM_025428 & 0.00020453 & -1.36483 & 0.0323279 & -1.13343 \\
\hline Prodh & NM_011172 & 0.00014486 & -1.37179 & 0.00244581 & -1.2259 \\
\hline Scn4b & NM_001013390 & 0.00023462 & -1.37682 & 0.000852337 & -1.30052 \\
\hline Mfge8 & NM_008594 & $2.08 \mathrm{E}-05$ & -1.37831 & 0.000692342 & -1.21349 \\
\hline Coro7 & NM_030205 & 6.67E-05 & -1.38432 & 0.00279585 & -1.20125 \\
\hline Sepw1 & NM_009156 & $1.82 \mathrm{E}-05$ & -1.40384 & 0.0253801 & -1.1086 \\
\hline Dbn1 & NM_001177371 & $2.11 \mathrm{E}-05$ & -1.40845 & 0.00858213 & -1.14334 \\
\hline Tmem180 & NM_029186 & 0.00014683 & -1.42698 & 0.000279983 & -1.38194 \\
\hline Usp2 & NM_198092 & 0.00014512 & -1.42989 & 0.00221837 & -1.26405 \\
\hline Gli3 & NM_008130 & 0.00022823 & -1.43065 & 0.556116 & -1.03543 \\
\hline 4931432E15Rik & AK016500 & 0.00024738 & -1.44531 & 0.0204267 & -1.18537 \\
\hline $\mathrm{Cbx} 4$ & NM_007625 & 8.89E-05 & -1.45169 & 0.00311063 & -1.23961 \\
\hline Dbp & NM_016974 & $6.72 \mathrm{E}-05$ & -1.46159 & 0.00904331 & -1.18824 \\
\hline Adora2a & NM_009630 & $9.53 \mathrm{E}-05$ & -1.4806 & 0.000225044 & -1.41427 \\
\hline Scube3 & NM_001004366 & 7.74E-05 & -1.48138 & 0.000674508 & -1.33039 \\
\hline
\end{tabular}




\begin{tabular}{|l|l|r|r|r|r|}
\hline Ido1 & NM_008324 & $8.60 \mathrm{E}-05$ & -1.491 & 0.000193323 & -1.42693 \\
\hline 2310034C09Rik & NM_054100 & 0.00021332 & -1.53469 & 0.0723369 & -1.14898 \\
\hline Prosapip1 & NM_197945 & $4.22 \mathrm{E}-05$ & -1.54302 & 0.00484289 & -1.23125 \\
\hline Abca9 & NM_147220 & 0.00014309 & -1.55893 & 0.000478416 & -1.44957 \\
\hline Actn2 & NM_033268 & 0.00028576 & -1.60686 & 0.00746275 & -1.31761 \\
\hline Id4 & NM_031166 & $4.63 \mathrm{E}-05$ & -1.63309 & 0.000237216 & -1.47458 \\
\hline Cml5 & NM_023493 & 0.00010866 & -1.70492 & 0.000352385 & -1.56697 \\
\hline Xkrx & NM_183319 & 0.00029446 & -1.78082 & 0.0107005 & -1.36877 \\
\hline Fzd2 & NM_020510 & $9.62 \mathrm{E}-05$ & -1.98053 & 0.0703235 & -1.22046 \\
\hline
\end{tabular}

Table 5.2. List of genes obtained through ANOVA and FDR correction analysis. Genes contained within table were shown to be modulated by METH treatment and these effects were mitigated by

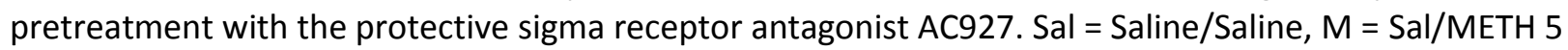
$\mathrm{mg} / \mathrm{kg}, \mathrm{AC}=\mathrm{AC9} 2710 \mathrm{mg} / \mathrm{kg} / \mathrm{Sal}, \mathrm{AM}=\mathrm{AC} 2710 \mathrm{mg} / \mathrm{kg} / \mathrm{METH} 5 \mathrm{mg} / \mathrm{kg}$.

\subsection{Discussion}

Data contained within the current chapter provides evidence that treatment with the selective sigma receptor antagonist AC927 mitigates specific gene changes associated with neurotoxic METH treatment in a rodent model. These genetic changes may be associated with the ability of AC927 and other sigma receptor antagonists to mitigate dopaminergic neurotoxicity associated with METH treatment in rodents.

Some of the genes included within the current lists have previously been shown to be increased by METH treatment (Thomas et al., 2004b). Many of the genes are known to be associated with neuroinflammatory processes, including gfap, cd68, cd44, and osmr (Baker et al., 2008; Hebert and O'Callaghan, 2000; Nakashima et al., 1999). These results provide initial evidence that AC927 treatment 
is associated with a reduction in neuroinflammation that is associated with neurotoxic METH treatment. A much more thorough and in depth collection of studies on the role of sigma receptor antagonists in mitigating METH-induced neuroinflammation is included in subsequent chapters of the current dissertation.

It should be emphasized that many of the genes included in the current lists are related to processes other than neuroinflammation. An analysis determining the role of many of these genes in various pathways and cascades associated with neuronal injury has yet to be conducted and it is possible that many of the genes above are involved in pathways associated with neurotoxicity. Furthermore, a comparison with microarray studies previously conducted and published aimed at determining the role of sigma-1 receptors in modulating gene expression has yet to be conducted (Tsai et al., 2012). A full comparison of gene alterations between this previous study and the current data set may provide further insight into which of the gene changes present within the current data set are mediated specifically through sigma-1 receptor modulation or whether sigma-2 receptors appear to be involved in modulating METH-induced genetic changes (Tsai et al., 2012).

Additionally, data contained within this chapter is representative of one time point after the administration of drug treatments. The genetic alterations determined within the current data set may not be representative of different time points after drug treatments and subsequent time courses should be run for further characterization of genetic changes that may be associated with the ability of sigma receptor antagonists to mitigate the neurotoxic effects of METH. 


\subsection{Contributions}

Matthew Robson conducted all in vivo experiments and RNA isolations. Wioletta Szeszel-

Fedorowicz, Ph.D. conducted the microarray experiments and Donquan Chen, Ph.D. analyzed all microarray data. 


\section{Chapter 6}

METH causes PERK-mediated endoplasmic reticulum stress in NG108-15 cells through a mechanism independent of sigma receptors 


\subsection{Introduction}

Chronic METH usage is associated with neurotoxicity in specific regions of the brain such as the striatum (Volkow et al., 2001b; Wang et al., 2004). These toxic consequences are believed to result in functional cognitive deficits and an increased risk of developing Parkinson's disease (Callaghan et al., 2010; Callaghan et al., 2012; Volkow et al., 2001b). Additionally, it has been shown that patients with greater nerve terminal degeneration have higher rates of relapse (Wang et al., 2012). Compounding these problems is the current paucity of viable pharmacotherapies aimed at treating the neurotoxic effects of METH usage.

The precise molecular mechanisms by which METH results in neurotoxicity are still being fully elucidated. One hypothesis is that signaling cascades associated with mitochondrial and endoplasmic reticulum (ER) function become disrupted and result in the initiation of signaling associated with cellular toxicity (Jayanthi et al., 2004; Krasnova and Cadet, 2009). Excesses of unfolded proteins, reactive oxygen species and reactive nitrogen species (ROS/RNS) and/or disruptions in inter-ER $\mathrm{Ca}^{2+}$ levels can disrupt ER function and result in the induction of ER stress signaling (Tabas and Ron, 2011).

The initiation of ER stress results in signaling through three distinct pathways, namely the activating transcription factor 6 (ATF6), inositol-requiring enzyme $1 \alpha$ (IRE-1 $\alpha)$ and PKR-like ER kinase (PERK) pathways (Ron and Walter, 2007; Walter and Ron, 2011). The induction of these pathways results in gene and protein expression alterations aimed at counteracting the ER stressor, whereas severe or prolonged ER stress results in the induction of apoptosis and cell death (Tabas and Ron, 2011). The PERK pathway is of particular importance as it mediates several crucial aspects of ER stress (Figure 5.1.). 


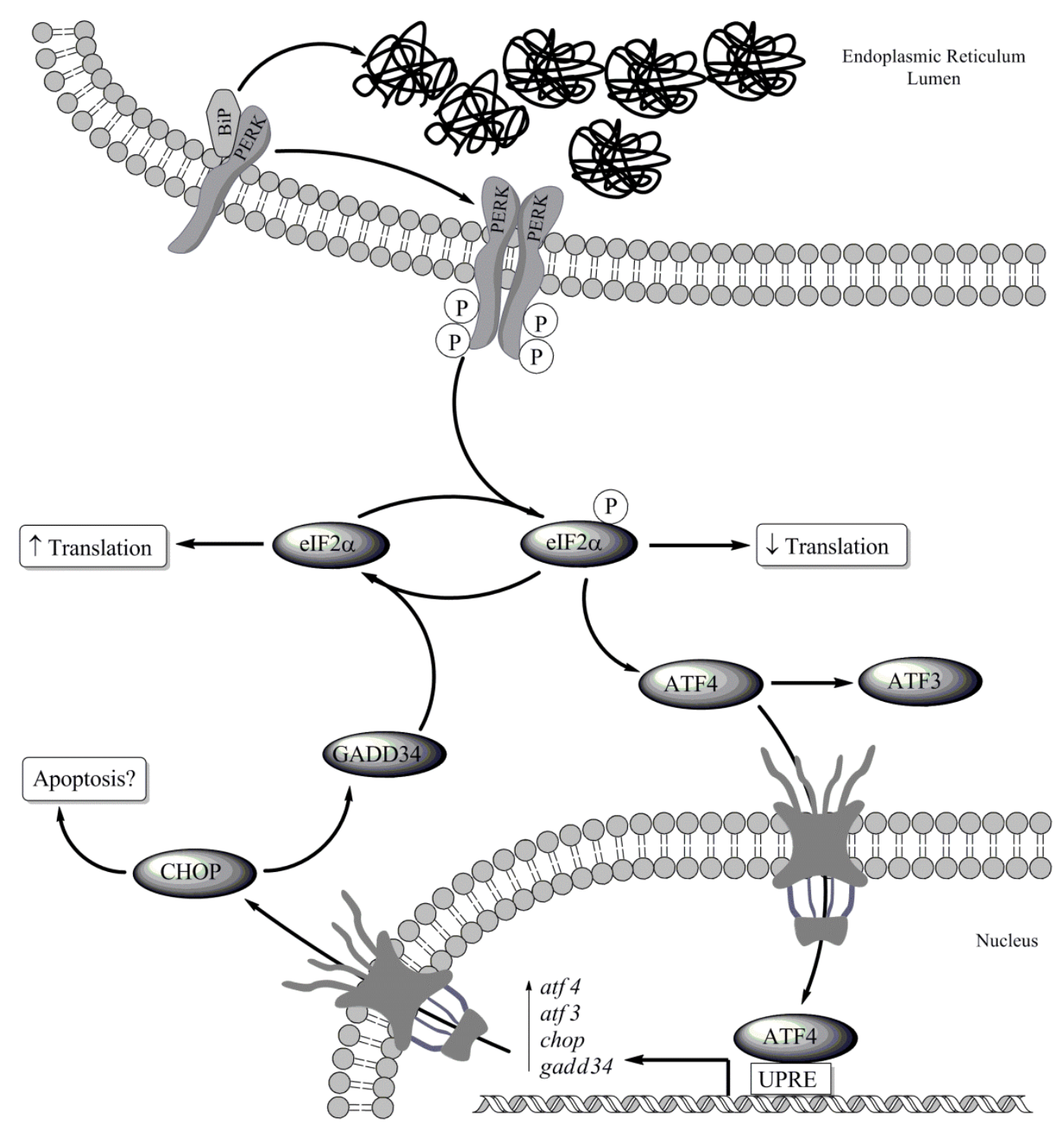

Figure 6.1. PERK-mediated ER stress pathway. Unfolded protein load and other ER perturbations result in the homodimerization of PERK at the ER membrane. Phosphorylated PERK, then phosphorylates elF $2 \alpha$, halting translation and activating ATF4 to cause the transcriptional upregulation of genes aimed at counteracting the perturbations present within the ER. If ER stressor persists, CHOP signaling can result in the activation of pathways relevant to apoptosis.

Initially, homodimerization and phosphorylation of PERK halts protein translation within the cell through the phosphorylation of eukaryotic initiation factor-2 $\alpha$ (elF $2 \alpha)$ to counteract unfolded protein load within the ER (Walter and Ron, 2011). Secondly, transcriptional upregulation of transcription factors such as activating transcription factor 3 (ATF3) and activating transcription factor 4 (ATF4) induce the upregulation of genes aimed at counteracting increases in unfolded protein load (Walter and Ron, 
2011). Additionally, PERK activation of ATF4 is a requirement for the upregulation of C/EBP homologous protein (CHOP) (Szegezdi et al., 2006). CHOP is believed to be a primary mediator of ER stress-induced apoptosis and has been implicated as a mediator of dopaminergic neurotoxicity (Gorman et al., 2012; Silva et al., 2005).

The induction of ER stress by METH has been reported in regions of the brain affected by METH neurotoxicity (Beauvais et al., 2011; Hayashi et al., 2010; Jayanthi et al., 2009). Specifically, the upregulation of genes involved in PERK-mediated ER stress signaling such as atf3, atf4, chop and protein phosphatase 1 regulatory subunit 15A (gadd34) was found along with a concomitant increase in CHOP protein levels (Beauvais et al., 2011). Importantly, it appears that CHOP upregulation is correlated with the neurotoxic actions of METH, as METH fails to upregulate CHOP in the absence of neurotoxicity (Takeichi et al., 2012). These results provide evidence that the induction of PERK-mediated ER stress may be associated with the neurotoxic actions of METH in vivo; however, systematic studies linking PERK-mediated ER stress to the neurotoxic actions of METH are currently lacking.

Sigma receptors have been proposed as potential targets for therapies aimed at treating the neurotoxic effects of METH. Sigma receptor antagonists, including 6-acetyl-3-(4-(4-(4fluorophenyl)piperazin-1-yl)butyl)benzo[d]oxazol-2(3H)-one (SN79), have been shown to attenuate $\mathrm{METH}$-induced neurotoxicity in vivo and in vitro by an as of yet unknown signaling mechanism (Kaushal et al., 2012a; Kaushal et al., 2012b; Matsumoto et al., 2008). Currently, there are two known subtypes of sigma receptors, denoted sigma-1 and sigma-2. Sigma-1 receptors are ligand-activated ER chaperone proteins that reside at the mitochondrial associated ER membrane (Hayashi and Su, 2007b).

Overexpression and knockdown of sigma-1 receptors has been shown to modulate cellular responses to ER stress in vitro, including phosphorylation levels of PERK elicited by an ER stressor (Hayashi and Su, 2007b). 
The ability of sigma-1 receptors to modulate ER stress responses led us to hypothesize that the ability of sigma receptor antagonists to mitigate METH-induced cellular toxicity may be due to an ability to modulate PERK-mediated ER stress elicited by the drug. The purpose of the current study was therefore to determine if METH causes PERK-mediated ER stress in an in vitro model of METH neurotoxicity and to determine if pretreatment with the prototypic sigma receptor antagonist, SN79 mitigates METH-induced PERK-mediated ER stress.

\subsection{Materials and Methods}

\subsubsection{Reagents and chemicals}

Phosphate-buffered saline (PBS), Dulbecco's modified Eagle's medium (DMEM), penicillin/streptomycin solution, hypoxanthine/aminopterin/thymidine (HAT) and TRIzol lysis reagent were obtained from Invitrogen (Carlsbad, CA). Fetal bovine serum (FBS), dimethylsulfoxide (DMSO), methamphetamine-HCl, Trizma base, glycine, sodium dodecyl sulfate (SDS) and tris-buffered saline (TBS) with Tween 20 were obtained from Sigma-Aldrich Chemicals (St. Louis, MO). Isopropanol and methanol were purchased from Fisher Scientific (Pittsburgh, PA). SN79 was synthesized in the laboratory of Dr. Christopher R. McCurdy (University of Mississippi), as previously described (Kaushal et al., 2011e).

\subsubsection{Cell culture}

The neuroblastoma X glioma hybridoma NG108-15 cell line was obtained from American Type Culture Collection (Manassas, VA) and cultured as previously described (Kaushal et al., 2012a). Briefly, cells were maintained in $75 \mathrm{~cm}^{2}$ (T75) culture flasks (Costar; Corning Life Sciences, Lowell, MA) at $37^{\circ} \mathrm{C}$ in high glucose DMEM with 10\% FBS, penicillin/streptomycin and HAT in a humidified incubator containing $5 \% \mathrm{CO}_{2}$. Cells were plated and grown in complete medium in 12-well CellBind tissue culture plates 
(Costar; Corning Life Sciences) for all quantitative real-time polymerase chain reaction (PCR) studies and 6-well CellBind tissue culture plates for protein level analysis. Cells were allowed to become approximately $80 \%$ confluent and medium was replaced with differentiation medium (DMEM containing 0.5\% FBS, penicillin/streptomycin, HAT and $1 \%$ DMSO) and cells were allowed to differentiate for an additional 3-4 days prior to undergoing respective treatments.

\subsubsection{Real-time PCR}

Differentiated NG108-15 cells cultured as described above underwent respective treatments and RNA isolations were conducted by removing media and adding TRIzol cell lysis reagent to extract total RNA according to manufacturer's instructions (Invitrogen, Carlsbad, CA). For antagonist experiments, cells were treated with either vehicle (media) or SN79 (100 nM) 15 min prior to adding METH (1000 $\mu \mathrm{M})$. The METH concentration was determined from previous toxicity experiments using differentiated NG108-15 cells (Kaushal et al., 2012a) and current dose response studies specifically related to ER stress. SN79 concentration (100 nM final concentration) was selected based on prior experiments utilizing this compound and cell line in which SN79 was shown to block cellular toxicity to METH at a large range of concentrations in differentiated NG108-15 cells (Kaushal et al., 2011c).

The quality and quantity of RNA was assessed using a spectrophotometer (Biochrom, Cambridge, UK). To synthesize cDNA, $1 \mu \mathrm{g}$ of RNA was used in conjunction with high capacity Superscript-H cDNA reverse transcription kits (Applied Biosystems, Foster City, CA). Respective cDNA was then used as a template for TaqMan quantitative real-time PCR with oligonucleotide primer sets specific for 18S rRNA (Hs99999901_s1) as an endogenous control and ATF3 (Mm00476032_m1), ATF4 (Mm00515324_m1), CHOP (Mm00492097_m1) and GADD34 (Mm01205601_g1) (Applied Biosystems, Foster City, CA) as PERK-mediated ER stress gene targets. All samples were run using TaqMan real-time PCR universal master mixture (Applied Biosystems) for a total of 45 cycles using a StepOnePlus real-time 
PCR system (Applied Biosystems). The difference in cycles to threshold for each sample as compared to the respective $18 \mathrm{~S}$ endogenous control for each sample was recorded. Threshold was set at 0.2 for all gene probes tested. The change in the difference in cycles to threshold method ( $\Delta \Delta C_{T}$ method) was used to calculate relative quantities of each gene in each respective sample obtained from NG108-15 cells.

\subsubsection{Western blots}

Differentiated NG108-15 cells cultured and treated as described above underwent protein isolations using TRIzol cell lysis reagent according to manufacturer's instructions (Invitrogen). The protein concentration of each sample was measured using a Coomassie Plus Bradford Assay Kit (Pierce, Rockford, IL) which is based upon a modified form of the Bradford assay (Bradford, 1976). Samples were run using $30 \mu \mathrm{g}$ of protein/well using Tris- $\mathrm{HCl} 12 \%$ pre-cast 15 -well gels (Bio-Rad, Hercules, $\mathrm{CA}$ ) in combination with 5X Lammeli sample buffer. Gels were run using a mini-PROTEAN system (Bio-Rad) and gels were equilibrated for 15 min in Towbin's buffer prior to transfer to polyvinylidene fluoride (PVDF)

membranes (Bio-Rad) using semi-dry electrophoretic transfer cells (Bio-Rad). Membranes were blocked using 5\% fat-free milk/TBS-T for $2 \mathrm{~h}$ at room temperature. Membranes were incubated with primary CHOP antibody (Cell Signaling, Danvers, MA) at a concentration of $1: 1,000$ overnight at $4^{\circ} \mathrm{C}$. Anti-rabbit IgG horseradish peroxidase (HRP)-linked antibody (Cell Signaling) was used at a concentration of 1:2,000 with gentle shaking for $2 \mathrm{~h}$. Imaging was conducted using LumiGLO chemiluminescent substrate (Cell Signaling) according to manufacturer's instructions. HRP-conjugated $\beta$-actin rabbit mAB (Cell Signaling) was used as an endogenous control for all respective samples at a concentration of 1:10,000 and incubated for a period of $1 \mathrm{~h}$. Molecular weight determination was conducted using a biotinylated protein ladder (Cell Signaling). Images were converted to 8-bit and analyzed using densitometry with background subtraction and normalized to $\beta$-actin using ImageJ software $(\mathrm{NIH}$; http://rsbweb.nih.gov/ij/). 


\subsubsection{Statistical analysis}

Statistical analysis consisted of one-way or two-way analysis of variance (ANOVA), followed by post hoc Tukey's or Bonferroni's test where applicable using GraphPad Prism 5 (GraphPad Software, San Diego, CA).

\subsection{Results}

\subsubsection{METH treatment results in transcriptional upregulation of PERK-mediated ER stress genes}

METH treatment dose-dependently increased the expression of PERK-mediated ER stress genes at all three time points tested $(6,12$, and $24 \mathrm{~h})$. Two-way ANOVA revealed that METH treatment resulted in a dose dependent difference in atf3 mRNA expression $(p<0.0001)$. Post hoc Bonferroni's analysis revealed that METH treatment increased atf3 expression at $6 \mathrm{~h}$, at concentrations of 300 and $1000 \mu \mathrm{M}(\mathrm{t}=4.41, \mathrm{p}<0.001$ and $\mathrm{t}=7.58, \mathrm{p}<0.001$, respectively). Additionally, atf3 mRNA expression was elevated at 12 and $24 \mathrm{~h}$ post-treatment at these two concentrations $(t=3.18, p<0.05, t=4.17, p<$ 0.01 and $t=5.19, p<0.001, t=6.27, p<0.001$ for 300 and $1000 \mu \mathrm{M}$ at 12 and $24 \mathrm{~h}$, respectively) (Figure 6.2.A.).

METH treatment also resulted in significant dose and time dependent increases in atf4 mRNA expression. Two-way ANOVA revealed significant differences in atf4 expression as a result of concentration, time and their interaction ( $p<0.0001, p<0.001$ and $p<0.05$, respectively). Bonferroni's post hoc tests revealed that METH treatment, at concentrations of 300 and $1000 \mu \mathrm{M}$, significantly increased atf4 mRNA expression at $6 \mathrm{~h}(\mathrm{t}=3.99, \mathrm{p}<0.01$ and $\mathrm{t}=9.33, \mathrm{p}<0.001$, respectively $)$, as compared to vehicle-treated controls. In addition, METH $1000 \mu \mathrm{M}$ resulted in significant increases in 
atf4 mRNA expression 12 and 24 h post-treatment (Figure 6.2.B.) $(\mathrm{t}=4.27, \mathrm{p}<0.001$ and $\mathrm{t}=8.50, \mathrm{p}<$ 0.001 , respectively).
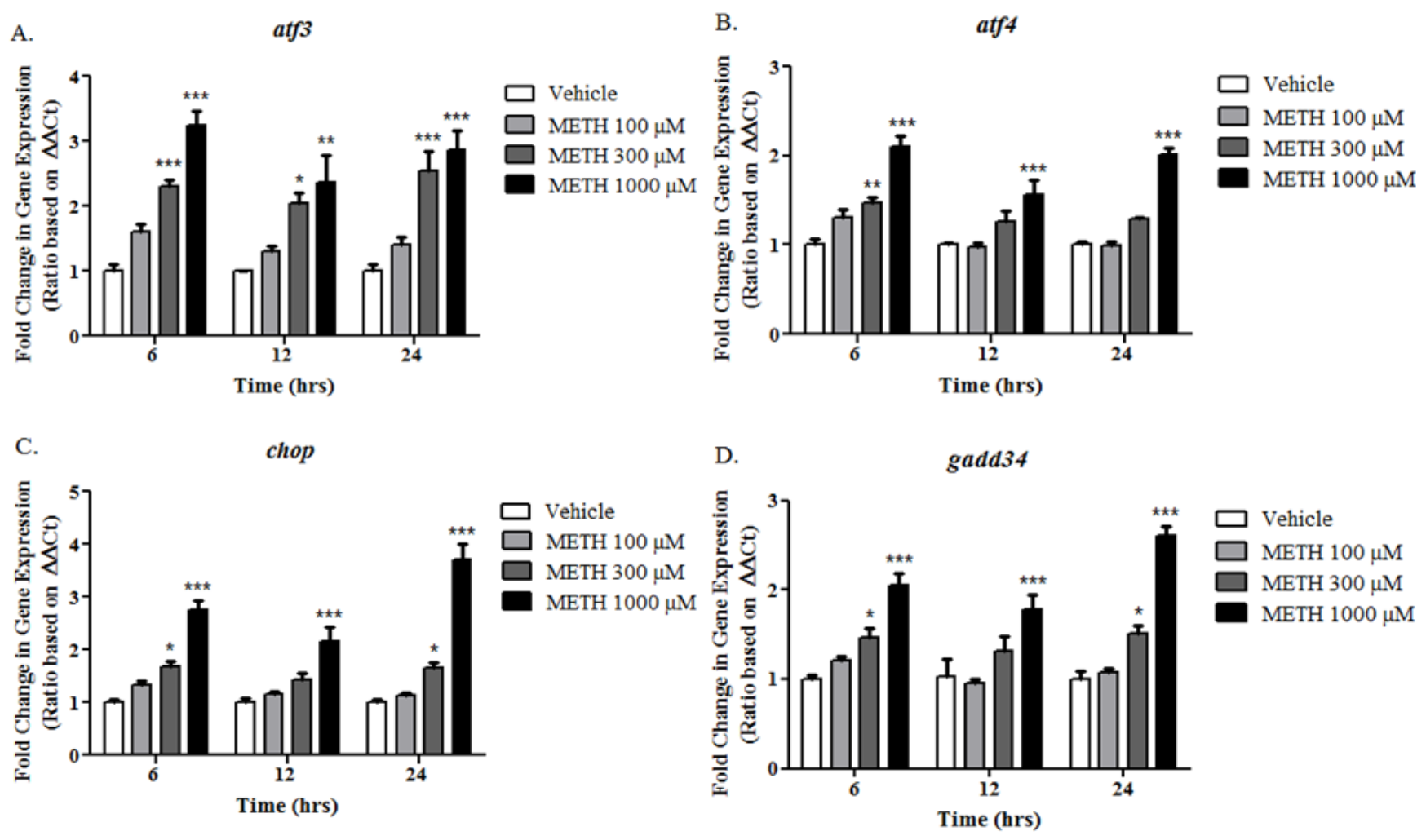

Figure 6.2. METH treatment causes an upregulation of genes involved in PERK ER stress in NG108-15 cells. (A) METH increases atf3 mRNA expression at 6,12 and 24 hrs post treatment $\left({ }^{*} p<0.05, * * p<\right.$ $0.01, * * * p<0.001$; vs. vehicle control). (B) METH treatment causes a significant upregulation of atf4 mRNA expression at 6, 12 and 24 hrs post-treatment (**p<0.01, ${ }^{* *} \mathrm{p}<0.001$; vs. vehicle control). (C) METH treatment results in significant upregulation of chop, a gene implicated in ER stress-induced apoptosis at all three time points as compared to vehicle treated controls $\left({ }^{*} p<0.05,{ }^{* * *} p<0.001\right)$. (D) gadd34 expression, another PERK-mediated ER stress gene, was upregulated by METH 6, 12 and 24 hrs post-treatment $(* p<0.05, * * * p<0.001$; vs. vehicle control).

METH treatment also increased the expression of chop, a downstream mediator of the PERK ER stress pathway. Two-way ANOVA revealed a significant dose and time effect of METH treatment on chop expression, as well as their interaction $(p<0.0001, p<0.01$ and $p<0.001$, respectively). Bonferroni's post hoc analysis revealed that METH treatment at concentrations of 300 and $1000 \mu \mathrm{M}$ increased chop mRNA expression 6 and $24 \mathrm{~h}$ post-treatment as compared to vehicle controls $(\mathrm{t}=3.22, \mathrm{p}<0.05$ and $\mathrm{t}=$ 
$8.20, p<0.001$ at $6 h$ and $t=3.06, p<0.05$ and $t=12.69, p<0.001$ at $24 h$ for 300 and $1000 \mu M$, respectively). Twelve hours post-treatment with METH $(1000 \mu \mathrm{M})$ resulted in a significant increase in chop mRNA expression $(t=4.80, p<0.001)$ (Figure 6.2.C.).

Additionally, gadd34 mRNA expression was determined at various time points after METH treatment. Two-way ANOVA revealed a significant dose, time and interaction effect of METH treatment on gadd34 expression ( $p<0.0001, p<0.01$ and $p<0.05$, respectively). Similar to METH-induced increases in chop expression, METH was found to increase gadd34 mRNA expression $6 \mathrm{~h}$ post-treatment, at concentrations of 300 and $1000 \mu \mathrm{M}$ (Bonferroni's post hoc tests, $\mathrm{t}=2.94, \mathrm{p}<0.05$ and $\mathrm{t}=6.56, \mathrm{p}<$ 0.001). Additionally, these concentrations increased gadd34 expression $24 \mathrm{~h}$ post-METH treatment $(\mathrm{t}=$ 3.20, $\mathrm{p}<0.05$ and $\mathrm{t}=10.00, \mathrm{p}<0.001$ for 300 and $1000 \mu \mathrm{M}$, respectively). The $1000 \mu \mathrm{M}$ concentration of METH also increased gadd34 mRNA expression $12 \mathrm{~h}$ post-treatment $(t=4.68, p<0.001)$ (Figure 6.2.D.).

\subsubsection{METH treatment increases CHOP protein expression}

Due to its involvement in toxicity elicited by PERK ER stress pathway activation, CHOP was selected for further protein level analysis of PERK-mediated ER stress activation by METH in NG108-15 cells. One-way ANOVA revealed a significant effect of $\mathrm{METH}$ treatment on $\mathrm{CHOP}$ expression $(\mathrm{F}[3,23]=$ $5.98, \mathrm{p}<0.01)$. Post hoc Dunnett's multiple comparison tests revealed that METH $(1000 \mu \mathrm{M}) 24 \mathrm{~h}$ posttreatment resulted in a significant increase in CHOP protein levels $(q=3.94, p<0.01)$ (Figure 6.3.). 


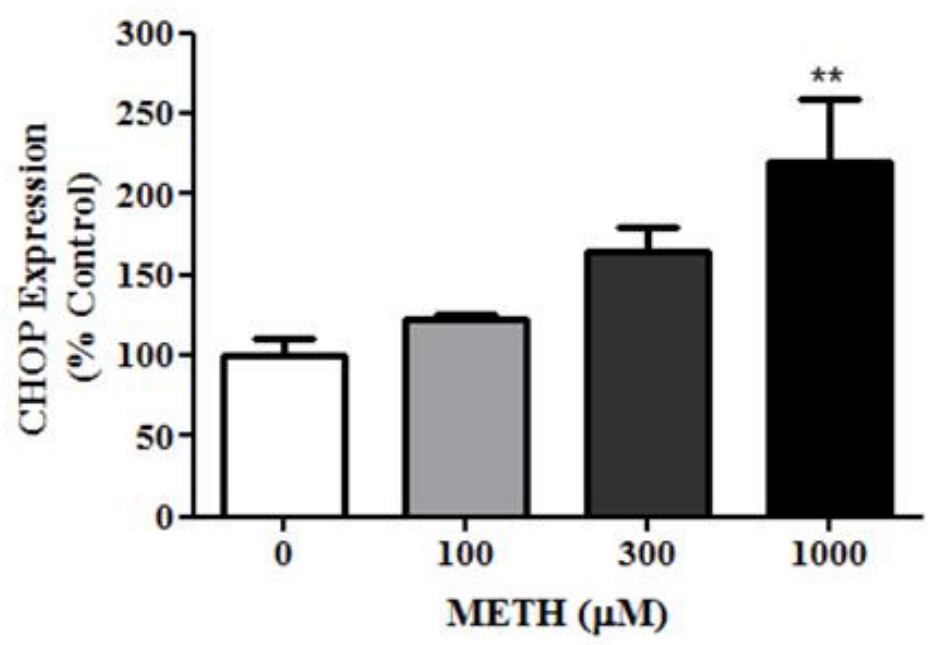

CHOP

\section{$\beta$-Actin}

Figure 6.3. METH treatment results in a dose dependent increase in CHOP protein expression $24 \mathrm{~h}$ posttreatment $\left({ }^{* *} p<0.01 ;\right.$ vs. vehicle control).

\subsubsection{SN79 + METH and the expression of PERK-mediated ER stress genes}

A single timepoint of $6 \mathrm{~h}$ was selected for use in experiments described below utilizing SN79, as METH $(1000 \mu \mathrm{M})$ alone was found to significantly increase expression levels of PERK-mediated ER stress genes at this early timepoint. Additionally, we have previously shown that at a later timepoint of $24 \mathrm{~h}$, METH $(1000 \mu \mathrm{M})$ results in significant cellular toxicity, an effect mitigated by treatment with SN79 (100 nM) (Kaushal et al., 2012a; Kaushal et al., 2011c; Kaushal et al., 2012b). Despite an ability to mitigate the neurotoxic effects of METH in both in vivo and in vitro models (Kaushal et al., 2011c; Kaushal et al., 2012b), SN79 treatment was found to have little effect on METH-induced PERK-mediated ER stress. Oneway ANOVA revealed significant differences between treatment groups in atf3 expression $(F[3,23]=$ 8.98, $\mathrm{p}<0.001)$. As depicted in Figure 6.4.A, METH $(1000 \mu \mathrm{M})$ treatment resulted in a significant increase in atf3 mRNA expression $6 \mathrm{~h}$ post-treatment (Tukey's test, $q=4.39, p<0.05)$. SN79 (100 nM) 
pretreatment had no effect on METH-induced increases in atf3 expression (n.s.), although cells treated with SN79 in combination with METH did significantly differ from vehicle treated controls $(q=5.96, p<$ 0.01). Additionally, SN79 displayed no effects on its own (n.s.) (Figure 6.4.A.).

SN79 treatment also had no effect on METH-induced chop mRNA expression. One-way ANOVA revealed significant differences between treatment groups $(F[3,23]=14.22, p<0.0001)$. METH treatment $(1000 \mu \mathrm{M})$ increased chop mRNA expression, as compared to vehicle-treated controls (Tukey's test, $q=4.95, p<0.05)$. SN79 treatment had no effect on METH-induced increases in chop mRNA expression (n.s.). Treatment with SN79 alone had no significant effects on chop mRNA expression as compared to vehicle-treated controls (n.s.) (Figure 6.4.B.).

One-way ANOVA revealed significant differences between treatment groups in gadd34 mRNA expression $(F[3,23]=13.89, p<0.0001)$. Post hoc analysis found that METH treatment $(1000 \mu \mathrm{M})$ increased gadd34 expression as compared to vehicle-treated controls $(q=5.19, p<0.01)$. SN79 in combination with METH increased expression of gadd34 mRNA, as compared to vehicle-treated controls $(q=8.10, p<0.001)$; however, SN79 treatment had no effect on METH-induced increases in gadd34 expression (n.s.) (Figure 6.4.C.).

Interestingly, SN79 pretreatment increased atf4 mRNA expression as compared to METH alone or vehicle-treated cells. One-way ANOVA revealed significant differences between treatment groups $6 \mathrm{~h}$ post-treatment in atf4 expression $(F[3,22]=13.59, p<0.0001)$. SN79 in combination with METH significantly increased atf4 expression, as compared to vehicle alone and METH alone treated cells (Tukey's multiple comparison tests, $q=8.29, p<0.001$ and $q=4.57, p<0.05$, respectively). METH treatment $(1000 \mu \mathrm{M})$ was unable to significantly increase atf4 expression $6 \mathrm{~h}$ post-treatment as compared to vehicle controls, although this effect was trending towards statistical significance $(q=3.91$, n.s.) (Figure 6.4.D.). 
A.

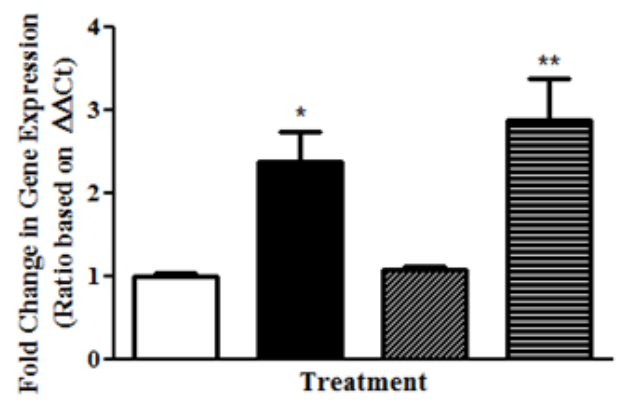

C.

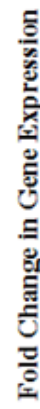

gadd34

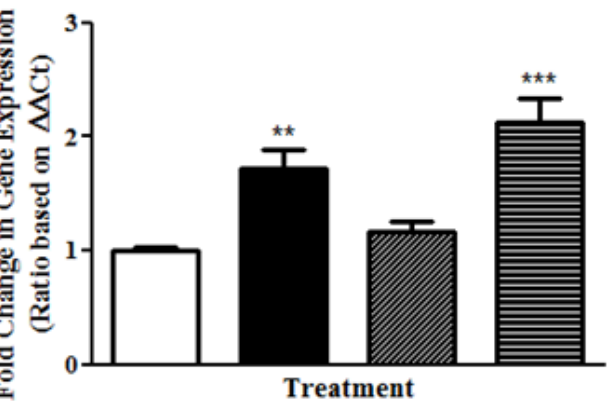

B.

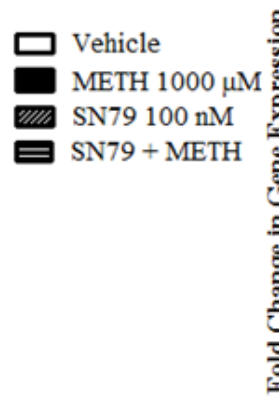

chop
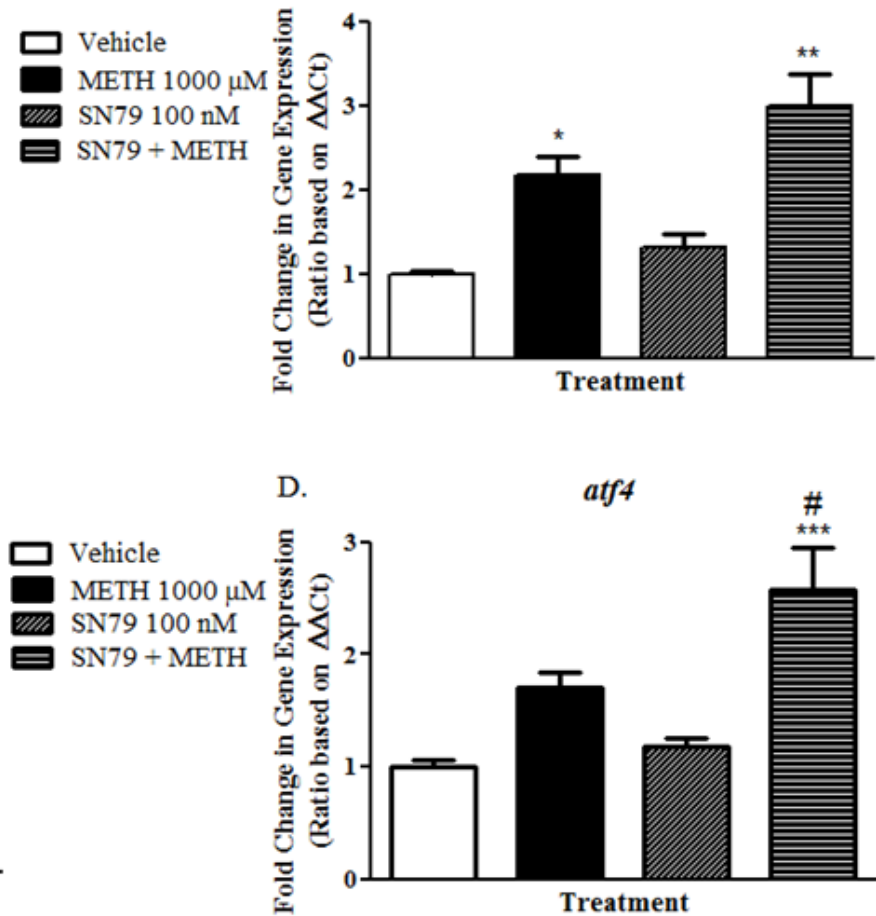

D.

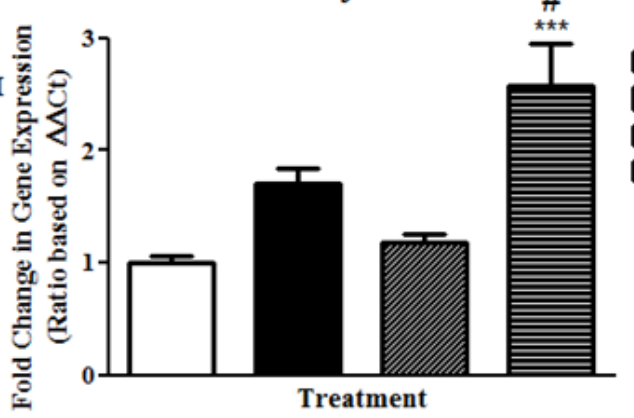

$\square$ Vehicle

METH $1000 \mu \mathrm{M}$ SN79 $100 \mathrm{nM}$ SN79+METH

Figure 6.4. SN79 treatment in combination with METH upregulates atf4 mRNA expression while having no effect on METH-induced upregulations of other PERK-mediated ER stress genes (A) SN79 treatment fails to alter METH-induced increases in atf3 mRNA expression $6 \mathrm{~h}$ post-treatment $\left({ }^{*} p<0.05,{ }^{* *} p<0.01\right.$; vs. vehicle control). (B) METH-induced increases in chop expression are not altered by SN79 treatment $\left({ }^{*} p<0.05,{ }^{* *} p<0.01\right.$; vs. vehicle control). (C) Similarly, METH-induced increases in gadd34 expression are unaltered by SN79 treatment ( ${ }^{* *} p<0.01, * * * p<0.001$; vs. vehicle control). (D) SN79 pretreatment significantly increases atf4 expression in NG108-15 cells, as compared to METH alone $\left({ }^{* *} \mathrm{p}<0.001\right.$; vs. vehicle control; \#p<0.05; vs. METH $1000 \mu \mathrm{M}$ ).

\subsubsection{SN79 + METH CHOP protein expression}

To evaluate potential regulation of CHOP by SN79 at the translational level, NG108-15 cells were treated with either vehicle, METH $(1000 \mu \mathrm{M})$, SN79 $(100 \mathrm{nM})$ or SN79 + METH for a period of $24 \mathrm{~h}$. One-way ANOVA found significant differences between treatment groups in CHOP protein expression $(F[3,23]=47.34, p<0.0001)$. Similar to experiments described above and as shown in Fig. 6.4., METH increased CHOP expression $24 \mathrm{~h}$ post-treatment as compared to vehicle controls (Tukey's test, q = 13.09, $p<0.001)$. SN79 pretreatment however, was unable to mitigate METH-induced increases in CHOP expression (n.s.) (Figure 6.5.). 


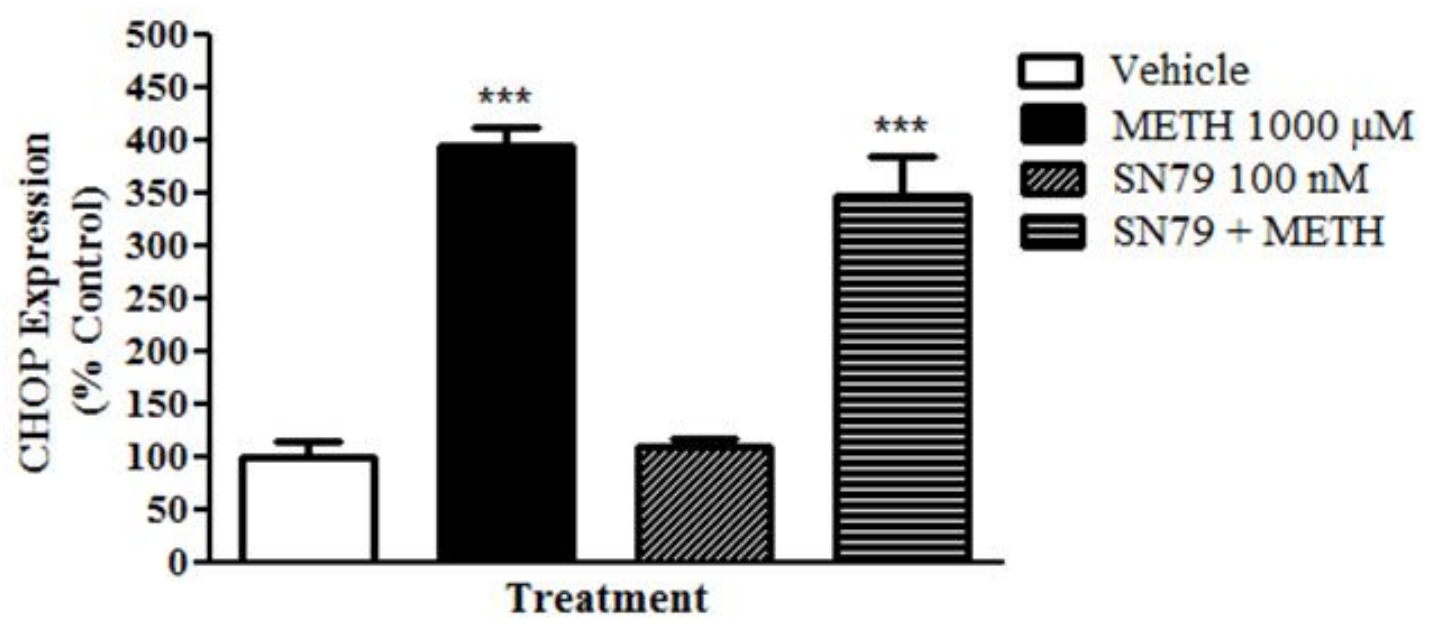

CHOP

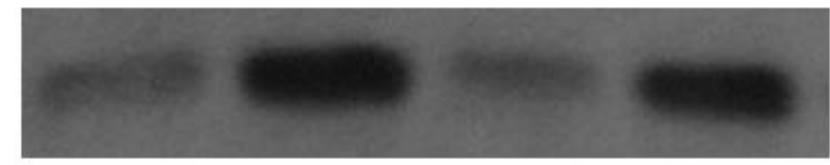

$\beta$-Actin

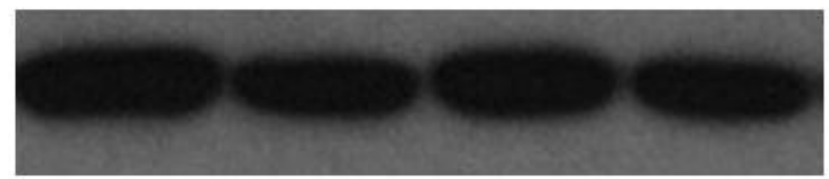

Figure 6.5. Treatment with the sigma receptor antagonist SN79 does not alter METH-induced increases in CHOP expression. METH $(1000 \mu \mathrm{M})$ treatment results in a significant upregulation of CHOP $24 \mathrm{~h}$ posttreatment in NG108-15 cells. SN79 pretreatment is unable to significantly alter METH-induced increases in CHOP expression ( ${ }^{* *} \mathrm{p}<0.001$, vs. vehicle control; n.s., SN79 + METH vs. METH).

\subsection{Discussion}

The current study shows that METH exposure results in PERK-mediated ER stress in an in vitro model of METH neurotoxicity. METH increases mRNA expression of atf3, atf4, chop and gadd34, with all four previously implicated in PERK-mediated ER stress signaling. Additionally, METH increases the expression of $\mathrm{CHOP}$, a protein involved in apoptosis elicited by prolonged ER stress. Furthermore, these effects occur on a time and concentration scale corresponding to earlier reported apoptosis by METH in NG108-15 cells (Kaushal et al., 2012a). Together, the data indicate that METH elicits a substantial increase in PERK-mediated ER stress signaling leading to enhanced CHOP expression, and subsequent ER stress-induced apoptosis. 
The initiation of PERK ER stress initially represents a protective measure as cells attempt to cope with increases in unfolded protein loads, changes in ER Ca ${ }^{2+}$ levels, or altered redox status (Lai et al., 2007; Walter and Ron, 2011). However, prolonged stimulation initiates pro-apoptotic signaling by upregulating proteins such as CHOP. It is known that for CHOP protein to be upregulated, the ATF4 arm of the PERK ER stress pathway must be active (Szegezdi et al., 2006). Furthermore, in the latent, apoptotic phases of ER stress, the PERK arm involving CHOP is active (Lin et al., 2007).

A role for CHOP in ER stress-induced apoptosis is supported by several earlier studies. Knockout of CHOP in mouse fibroblasts reduces ER stress-induced apoptosis (Zinszner et al., 1998). CHOP knockout also significantly reduces apoptosis of dopaminergic neurons in 6-hydroxydopamine (6-OHDA) treated rats (Silva et al., 2005). In neuronal cells in vitro, PERK-mediated ER stress increases ATF4mediated CHOP expression and apoptosis (Galehdar et al., 2010). Thus, the ability of METH to act through PERK-mediated ER stress signaling for a prolonged period at concentrations that result in apoptosis provides evidence that METH exerts toxic responses through this ER stress signaling cascade.

Further implicating PERK-mediated ER stress in the toxic effects of METH, PERK-mediated ER stress occurs in the striatum of rats whom have undergone a neurotoxic dosing paradigm of the drug (Beauvais et al., 2011; Jayanthi et al., 2009). These changes are correlated with the neurotoxic actions of the drug, as CHOP upregulation by METH occurs only in the presence of neurotoxicity (Takeichi et al., 2012). For example, low-dose METH pretreatments that mitigate METH-induced neurotoxicity (as measured by tyrosine hydroxylase levels) also block increases in CHOP expression (Takeichi et al., 2012). In vitro, one prior report has shown an induction of CHOP expression by METH in CATH.a cells, a dopaminergic cell line, after exposure for at least $24 \mathrm{~h}$ (Irie et al., 2011). This report however, examined only CHOP expression and neglected other participating members of the PERK-mediated ER stress pathway. The current study extends and confirms these earlier observations by showing induction of multiple components of PERK ER stress by METH in a cell line on a time scale similar to that obtained 
through in vivo studies utilizing rodents (Beauvais et al., 2011) and time and concentration scale corresponding to toxicity elicited by METH in vitro (Kaushal et al., 2012a).

CHOP is not the sole member of the PERK ER stress pathway implicated in ER stress apoptosis. The induction of GADD34 is another way in which METH may regulate ER stress-induced apoptosis, as overexpression of GADD34 can initiate and potentiate apoptosis (Adler et al., 1999). It is possible that METH-induced increases in GADD34 expression increases unfolded protein load by dephosphorylating elF2 $\alpha$, thereby reinstating translation and increasing apoptosis (Marciniak et al., 2004; Tsaytler et al., 2011).

The convergence of downstream mediators related to PERK-mediated ER stress signaling such as CHOP and GADD34 and apoptosis signaling cascades may be a significant contributing factor in the ability of METH to cause apoptosis. Increases in CHOP expression can decrease the anti-apoptotic protein Bcl-2 (McCullough et al., 2001). Interestingly, METH can also alter Bcl-2/BAX protein ratios in regions of the brain affected by its neurotoxic actions (Beauvais et al., 2011; Imam et al., 2001; Jayanthi et al., 2001). The upregulation of PERK-mediated ER stress in these brain regions by METH may alter the ratios of anti-apoptotic and apoptotic proteins, thereby leading to neurotoxicity.

The induction of PERK-mediated ER stress in vivo appears dependent on dopaminergic signaling as pretreatment with the $\mathrm{D} 1$ receptor antagonist $\mathrm{SCH} 23390$ attenuates gene upregulation of atf3, atf4, chop and gadd34 by METH in the striatum (Beauvais et al., 2011). Additionally, pretreatment with the D2 receptor antagonist raclopride exerts moderate inhibitory effects on $\mathrm{METH}$-induced increases in CHOP expression in the striatum, further implicating dopamine receptor signaling in these effects (Beauvais et al., 2011). Sigma-1 receptors have been shown to modulate dopamine D1 signaling in response to psychostimulant-mediated activation; however, the role of this interaction in mediating cellular stress responses has yet to be determined (Navarro et al., 2010). 
Thus, the second aim of the current study was to determine if ER stress elicited by METH is invoked through signaling involving sigma receptors. Previous work shows that sigma receptor antagonists mitigate METH-induced neurotoxicity in vivo and in vitro through an unknown signaling mechanism. SN79, the prototypic sigma receptor antagonist utilized in the current study, has previously been shown to mitigate METH-induced apoptosis, necrosis, caspase activation and ROS/RNS generation in NG108-15 cells (Kaushal et al., 2011c). We hypothesized that the neuroprotective effects of sigma receptor antagonists against METH may also result from the modulation of ER stress signaling. Interestingly, pretreatment with SN79 increases atf4 mRNA expression as compared to METH alone; however, SN79 has no effect on expression levels of other PERK ER stress genes under similar conditions. The inability of a sigma receptor antagonist to modulate PERK-mediated ER stress elicited by METH is surprising. Overexpression of sigma-1 receptors has previously been shown to attenuate PERK phosphorylation, indicative of an upstream blockade of PERK-mediated ER stress, in response to the ER stressor thapsigargin in CHO cells (Hayashi and Su, 2007b). Thapsigargin functions, however, by depleting $\mathrm{ER} \mathrm{Ca}^{2+}$ stores and it may be possible that METH results in ER stress solely by altering the level of unfolded proteins within the ER irrespective of $\mathrm{ER} \mathrm{Ca}^{2+}$ levels. This is one potential explanation for the disparities between data presented here utilizing METH and reports using thapsigargin to induce PERKmediated ER stress.

Interestingly, the increase in atf4 mRNA expression as compared to METH alone could be a way for sigma receptor ligands to modulate PERK-mediated ER stress. This increase led us to believe that CHOP protein expression might be elevated at later time points. However, there was no difference in CHOP protein expression in METH samples pretreated with SN79 or those with vehicle. It is currently unclear why this occurred; however, ATF4 has recently been shown to play a crucial role in several aspects of cellular energy regulation. ATF4 is believed to be involved in lipid metabolism, responses to amino acid deprivation, and glucose metabolism in addition to its roles in ER stress (Seo et al., 2009; Siu 
et al., 2002; Wang et al., 2010). It is hypothesized that sigma-1 receptors, when activated, are able to increase energy production by modulating $\mathrm{Ca}^{2+}$ flux into mitochondria thereby altering cellular energy production (Hayashi et al., 2009). It is thus possible that sigma receptor modulation of atf4 expression alters cellular energetics and conveys protection against the toxic effects of METH.

In conclusion, the current study provides the first evidence that METH causes PERK-mediated ER stress an in vitro model of METH neurotoxicity. Sigma receptor modulation can have many effects on cellular signaling and death cascades (Meunier and Hayashi, 2010; Su and Hayashi, 2003; Su et al., 2010a), but does not appear to affect PERK-mediated ER stress by METH. Further studies to delineate the molecular mechanisms by which sigma antagonists mitigate the neurotoxic actions of METH and to evaluate the role of PERK-mediated ER stress in the toxic actions of METH are warranted.

\subsection{Contributions}

Matthew Robson conducted and analyzed all experiments contained within Chapter 5. 


\section{Chapter 7}

SN79, a sigma receptor ligand, blocks METH-induced microglial activation and cytokine upregulation 


\subsection{Introduction}

METH is an addictive psychostimulant that is currently the second most abused illicit substance in the world behind only Cannabis (Romanelli and Smith, 2006). METH exerts many of its behavioral and physiologic effects by modulating monoaminergic systems within the central nervous system (CNS). METH abuse has several associated side effects including mood disturbances, anxiety, severe dental problems and notably, neurotoxicity (Romanelli and Smith, 2006). Chronic METH usage has been shown in humans to result in dopaminergic damage in the striatum, an effect correlated to relapse rates during clinical addiction treatment (Volkow et al., 2001a; Volkow et al., 2001b; Wang et al., 2012). Recently, chronic abusers of METH were also found to have a greater risk of developing Parkinson's disease later in life, which is believed to stem from the neurotoxic consequences of the drug (Callaghan et al., 2010; Callaghan et al., 2012).

The precise mechanisms by which METH elicits neurotoxic effects are still being elucidated. There are several contributing factors that have been implicated in these effects, such as increased reactive oxygen and nitrogen species (ROS/RNS) generation, dopamine quinone formation, caspase and cell death signaling cascade activation, endoplasmic reticulum (ER) stress induction, and glutamatergic excitotoxicity (Cadet and Krasnova, 2009; Krasnova and Cadet, 2009). Additionally, microglial activation has been implicated in the neurotoxic effects of METH (Escubedo et al., 1998; Kelly et al., 2012; Kuhn et al., 2006; Thomas et al., 2004a; Yue et al., 2012).

Microglia are the resident macrophages of the CNS that function in maintaining homeostasis by sensing deviations from the normal brain environment. Upon activation by perturbations of their surrounding environment, microglia can undergo transformation to different response phenotypes, similar to peripheral macrophages (Perry et al., 2010; Saijo and Glass, 2011). These have classically been categorized as M1- and M2-type macrophage/microglia, with M1 microglia being associated with inflammation and degeneration and $\mathrm{M} 2$ being associated with regeneration or anti-inflammatory 
processes (Czeh et al., 2011; Mosser and Edwards, 2008). The classical activation of microglia (M1) is associated with an upregulation of a variety of cell surface proteins, release of pro-inflammatory cytokines, generation of ROS/RNS, and subsequent neuronal damage (Czeh et al., 2011; Mosser and Edwards, 2008; Perry et al., 2010), all of which have been shown to be increased in response to neurotoxic regimens of METH (Escubedo et al., 1998; Kuhn et al., 2006; O'Callaghan et al., 2008; Thomas et al., 2004a; Thomas et al., 2004c).

The activation of microglia by METH has been demonstrated in both human studies and preclinical rodent models (Sekine et al., 2008; Thomas et al., 2004a; Thomas et al., 2004c). Microglial activation by METH occurs in regions of the brain affected by the neurotoxic actions of the drug and these effects have been shown to be persistent and long lasting even after extended abstinence from the drug (Sekine et al., 2008). The ability of microglia to produce cytokines and reactive species that can compromise synaptic transmission and neuronal function make them logical contributors to METH neurotoxicity and intriguing targets for drug development.

Among the pro-inflammatory cytokines, members of the interleukin-6 (IL-6) family are of particular relevance in the context of METH neurotoxicity. It is believed that the release of cytokines (including IL-6-type cytokines) by activated microglia is relevant to the effects of several neurodegenerative disorders, representing mechanistic overlap between these diseases and the neurotoxic actions of METH (Cadet and Krasnova, 2009; Smith et al., 2012b). The upregulation of IL-6 and other members of this cytokine family have been shown in brain regions affected by the neurotoxic actions of METH (Goncalves et al., 2008; Kelly et al., 2012). Moreover, it has previously been shown that mice lacking IL-6 are protected against the neurotoxic actions of METH (Ladenheim et al., 2000), supporting a role of IL-6 in the neurotoxic actions of the drug. Furthermore, it is believed that molecular signaling cascades activated by IL-6-type cytokine signaling are involved in glial cell activation by METH (Hebert and O'Callaghan, 2000). 
Presently, there are no FDA approved medications aimed at treating any of the negative side effects of METH abuse, including neurotoxicity. Many potentially promising preclinical treatments have failed to provide clinically effective pharmacotherapies, including those targeting monoaminergic systems. Sigma receptors have recently emerged as potential targets for the production of novel therapeutics aimed at treating many of the negative effects associated with METH usage, including neurotoxicity (Robson et al., 2012; Rodvelt and Miller, 2010). There are currently two known subtypes of sigma receptors, denoted sigma-1 and sigma-2, and sigma receptor antagonists have been shown to mitigate METH-induced behavioral effects and hyperthermia in rodents (Matsumoto et al., 2008; Nguyen et al., 2005). Additionally, sigma receptor antagonists block METH-induced reductions in striatal dopamine and serotonin and their respective transporters in preclinical models of METH-induced neurotoxicity (Kaushal et al., 2012b; Seminerio et al., 2011). The ability of sigma receptor antagonists to mitigate the effects of METH on neuronal cells is well documented; however, it is currently unclear if sigma receptor antagonists also block METH-induced microglial activation.

Sigma receptors are found in microglial cells and sigma receptor ligands have been shown to modulate microglial activation in vitro and in vivo (Cuevas et al., 2011; Hall et al., 2009). Sigma receptor ligands have been shown to modulate several aspects of microglial activation including migration and cytokine release in response to various activators such as adenosine triphosphate (ATP) and lipopolysaccharide (LPS) (Cuevas et al., 2011; Hall et al., 2009). The sigma receptor ligand SR 31747 has also been reported to block peripheral increases in IL-6 expression in response to peripheral LPS and Staphylococcal enterotoxin B administration, although data from the CNS is currently lacking (Bourrie et al., 1996; Derocq et al., 1995). These studies provide evidence that sigma receptor ligands are capable of modulating the functionality of immune cells, including those present within the CNS. The purpose of the current study was therefore to determine if the putative sigma receptor antagonist and drug development candidate, 6-acetyl-3-(4-(4-(4-fluorophenyl)piperazin-1-yl)butyl)benzo[d]oxazol-2(3H)-one 
(SN79), mitigates microglial activation and cytokine upregulation elicited by METH in the striatum in a preclinical model of METH-induced neurotoxicity.

\subsection{Materials and Methods}

\subsubsection{Drugs and chemicals}

(+)-Methamphetamine hydrochloride was obtained from Sigma Aldrich (St. Louis, MO). SN79 was synthesized as previously described (Kaushal et al., 2011e) and provided by Dr. Christopher R. McCurdy (University of Mississippi, University, MS). All administered drugs were dissolved in sterile saline solution ( $0.1 \mathrm{ml} / 10 \mathrm{~g}$ body weight) (Teknova, Fisher Scientific, Pittsburgh, PA). All other chemicals were obtained from Sigma Aldrich (St. Louis, MO) unless otherwise specified.

\subsubsection{Animals}

Male, Swiss Wesbter mice (24-28 g; Harlan, Indianapolis, IN) were utilized for all experiments. Mice were housed in groups of five, on a 12:12 h light/dark cycle with food and water ad libitum. Mice were randomly assigned to their respective treatment groups for all experiments. Experiments were performed as approved by the Animal Care and Use Committee at West Virginia University.

\subsubsection{Repeated dosing paradigm}

Mice were randomly distributed for each experiment into one of four treatment groups: 1) Saline/Saline (0.1 ml/10 g body weight), 2) Saline/METH 5 mg/kg, 3) SN79 3 mg/kg/Saline, or 4) SN79 3 $\mathrm{mg} / \mathrm{kg} / \mathrm{METH} 5 \mathrm{mg} / \mathrm{kg}$. The first compound listed in each pair (Saline or SN79) was administered as a pretreatment 15 min prior to the second compound in each treatment group (Saline or METH). Each 
animal underwent four pretreatments/treatments at $2 \mathrm{~h}$ intervals as previously described (Kaushal et al., 2012b). All injections were administered intraperitoneally.

Core body temperature was measured $1 \mathrm{~h}$ after each treatment using a probe (Thermalert TH-S Monitor, Physitemp Instruments Inc., Clifton, NJ) inserted approximately $2.5 \mathrm{~cm}$ past the rectum into the colon. Body temperature was recorded after a stable temperature had been reached approximately 10 s post-probe insertion.

The METH dose ( $5 \mathrm{mg} / \mathrm{kg} \times 4$ ) was selected based upon previous dose response experiments assessing dopaminergic neurotoxicity in this specific model, where it has consistently been shown to result in significant dopaminergic deficits in the striatum (Matsumoto et al., 2008; Seminerio et al., 2012a). Similarly, the SN79 dose (3 mg/kg x 4) was selected due to previously reported dose response experiments assessing the ability of this compound to mitigate the striatal dopaminergic deficits elicited by METH (Kaushal et al., 2012b).

At various timepoints post-treatment (as measured from the last injection) bilateral striatum samples were collected, flash frozen in liquid nitrogen and stored at $-80^{\circ} \mathrm{C}$ for later use. Samples for protein level analysis were collected $72 \mathrm{~h}$ post-treatment and were collected after transcardial perfusion with phosphate buffered saline (PBS) followed by $4 \%$ paraformaldehyde. Time points were selected based upon time course experiments contained within this report (microglial markers and cytokine expression levels) and previous data reporting the timeline of METH-induced dopaminergic neurotoxicity and microglial activation in rodent models (Escubedo et al., 2005; Escubedo et al., 1998; Kita et al., 2000; Thomas et al., 2004c).

\subsubsection{Quantitative real-time PCR}

Total RNA was extracted from flash frozen striatum samples using Trizol reagent (Invitrogen, Grand Island, NY) according to manufacturer's instructions. The total RNA concentration for each sample 
was quantified by spectral absorption, and the purity of the sample checked to confirm that the $260 / 280$ ratio was in the range of 1.8-2.1. Samples of cDNA were prepared by reverse transcription using a high capacity reverse transcription kit (Applied Biosystems, Foster City, CA). Each sample reaction included MultiScribe TM Reverse Transcriptase and random primers, with thermal cycler conditions set as follows: step 1 at $25^{\circ} \mathrm{C}$ for $10 \mathrm{~min}$, step 2 at $37^{\circ} \mathrm{C}$ for $120 \mathrm{~min}$, step 3 at $85^{\circ} \mathrm{C}$ for $5 \mathrm{~s}$, and step 4 at $4^{\circ} \mathrm{C}$ for $10 \mathrm{~min}$.

For the PCR amplification, TaqMan ${ }^{\circledR}$ Universal PCR Master Mix and the following probes were obtained from Applied Biosystems (Foster City, CA): 18s (Hs99999901_s1) for use as an endogenous control gene, IL6 (Mm00446190_m1), OSM (Mm0119366_m1), CD68 (Mm03047343_m1) and LIF (Mm00434762_g1). The reaction mixture was prepared according to the manufacturer's instructions, with the following thermal cycling conditions: initial holding at $50^{\circ} \mathrm{C}$ for $2 \mathrm{~min}$ which is required for optimal AmpErase ${ }^{\circledR}$ UNG activity, followed by a first denaturing step at $95^{\circ} \mathrm{C}$ for 10 min, then 45 cycles at $95^{\circ} \mathrm{C}$ for $15 \mathrm{~s}$, and at $60^{\circ} \mathrm{C}$ for $1 \mathrm{~min}$. The data from the real-time PCR measurements were calculated using the $\Delta \Delta \mathrm{Ct}$ method. The threshold value was set at 0.2 and the threshold cycle (Ct value) of each gene was normalized to $18 \mathrm{~s}$ rRNA.

\subsubsection{Immunohistochemistry}

At $72 \mathrm{~h}$ post-treatment (after the last injection), animals were anesthetized and immediately perfused transcardially with PBS followed by $4 \%$ paraformaldehyde. The entire brain was removed and immediately placed in $4 \%$ paraformaldehyde for $24 \mathrm{~h}$. Following fixation, the brains were processed using a Tissue-Tek VIP 5 automatic tissue processor (Sakura Finetek, Torrance, CA) and then embedded in paraffin with a Tissue-Tek TEC 5 embedding console system (Sakura Finetek). Embedded tissues were sliced in $6 \mu \mathrm{m}$ sections using a Leica RM2235 microtome (Leica Microsystems, Buffalo Grove, IL), and slices mounted on glass slides for staining. All slides were heat-fixed and deparaffinized via a series of 
xylene and alcohol washes prior to immunohistochemical procedures. Classical (M1) and alternative (M2) labeling of microglia was conducted as previously described (Niino et al., 2010; Zhang et al., 2012). Briefly, tissues were incubated first in mouse anti-rat CD68 antibody (AbD Serotec, Kidlington, UK) at a dilution of 1:100 in 4\% horse serum in Dulbecco's phosphate buffered saline (DPBS) overnight. Sections were washed $3 x$ in DPBS and then incubated in a biotinylated anti-mouse IgG secondary antibody (Vector Laboratories, Burlingame, CA) diluted at 1:10,000 in 4\% horse serum in DPBS for $4 \mathrm{~h}$. Following incubation with a secondary antibody, alkaline phosphatase (Life Technology, Carlsbad, CA) diluted at 1:100 in Tris-bovine serum albumin (BSA) was applied for $1 \mathrm{~h}$. Following the incubation with alkaline phosphatase, the tissues were rinsed 3x in DPBS and then Fast Blue BB salt (Santa Cruz Biotechnology, Santa Cruz, CA) for 5 min. Tissues were again rinsed 3x in DPBS prior to incubation in mouse anti-rat CD163 (AbD serotec, Kidlington, UK) at a dilution of 1:100 in 4\% horse serum in DPBS overnight. Following incubation with the primary antibody, the tissues were washed $3 x$ in DPBS and then incubated in a biotinylated anti-mouse IgG secondary antibody (Vector Laboratories, Burlingame, CA) diluted at $1: 10,000$ in $4 \%$ horse serum in DPBS for $4 \mathrm{~h}$. Following incubation with the secondary antibody, the tissues were then incubated in avidin D-horseradish peroxidase (HRP) (Vector Laboratories, Burlingame, CA) diluted at 1:1,000 in DPBS for $1 \mathrm{~h}$. The chromagen solution, diaminobenzidine (DAB) (Vector Laboratories, Burlingame, CA), was applied for 5 min per manufacturer's instructions. The tissues underwent a final wash in xylene and were mounted using an antifade agent and cover slipped. The slides were sealed with acrylic and stored in the dark in a laboratory refrigerator. Staining for IBA-1, another marker of microglial activation, followed a similar approach but utilized anti-IBA-1 polyclonal antibody raised in rabbit (Wako, Richmond, VA). Sections were developed using Nova Red (Vector Laboratories, Burlingame, CA). 


\subsubsection{Statistical analysis}

Statistical analyses were conducted using analysis of variance (ANOVA). For significant effects, pairwise comparisons followed using post hoc Bonferroni's or Tukey's multiple comparisons tests. P < 0.05 was considered statistically significant.

\subsection{Results}

\subsubsection{Body temperature modulation}

Dosing regimens of METH previously found to be neurotoxic (Matsumoto et al., 2008; Seminerio et al., 2012a) significantly increased core body temperature in mice, as compared to saline-treated controls. Two-way repeated measures ANOVA revealed significant differences between treatment groups and time, as well as their interaction $(p<0.0001, p<0.0001$ and $p<0.0001$, respectively). Bonferroni's post hoc analyses revealed that METH $(5 \mathrm{mg} / \mathrm{kg} \times 4)$ treatment significantly increased body temperature as compared to saline at time points 2,3 and $4(t=5.16, p<0.001, t=9.45, p<0.001$ and $t$ $=10.39, p<0.001$, respectively) (Fig. 7.1.). SN79 pretreatment was also found to significantly attenuate METH-induced changes in body temperature at all four time points $(1: t=7.28, p<0.001 ; 2: t=8.50, p<$ $0.001 ; 3: t=8.93, p<0.001$ and $4: t=10.38, p<0.001$ ), while having no effects on its own as compared to saline-treated animals (n.s. for time points 1, 2, 3 and 4) (Fig. 7.1.). 


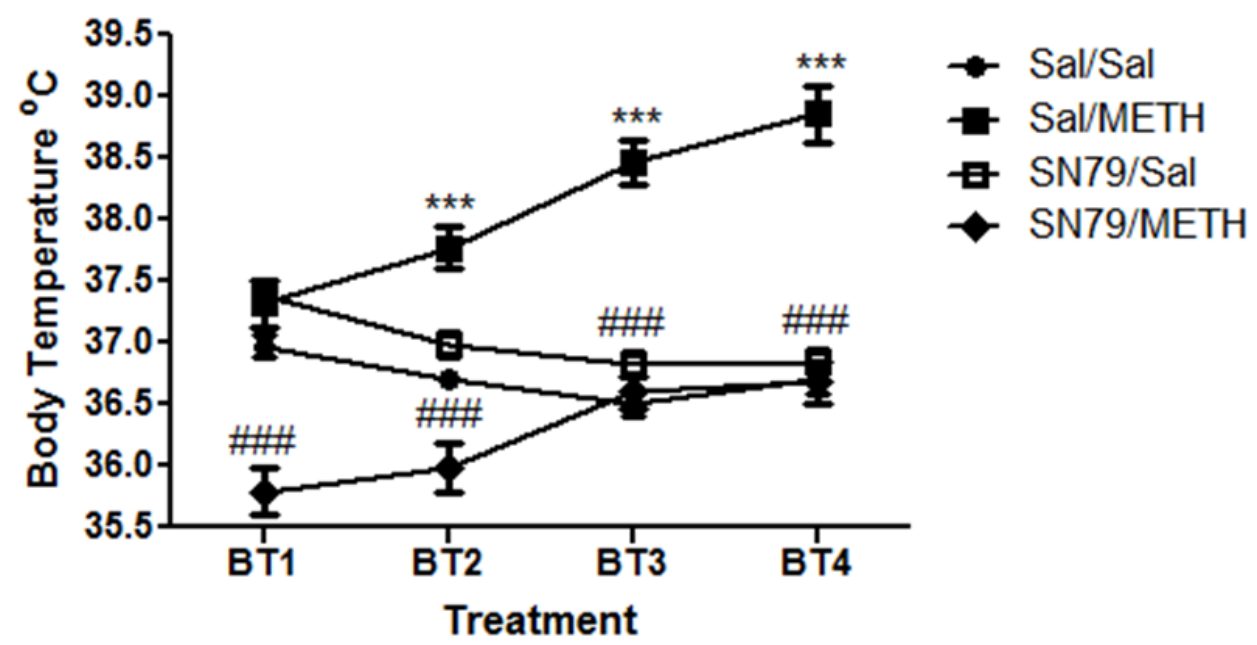

Figure 7.1. Repeated methamphetamine (METH, $5 \mathrm{mg} / \mathrm{kg} \times 4)$ treatment increased core body temperature as compared to saline (Sal), an effect mitigated by SN79 (3 mg/kg x 4) pretreatment. SN79 treatment also displayed no effects on basal body temperature alone. Two-way repeated measures ANOVA, followed by post hoc Bonferroni's multiple comparison tests; ${ }^{* * *} p<0.001$, Sal/Sal vs. Sal/METH; \#\#\# p<0.001, Sal/METH vs. SN79/METH. BT=body temperature.

\subsubsection{Markers of microglial activation}

CD68 expression is a commonly used marker of microglial activation due to CNS insult (Komohara et al., 2011; Li et al., 2009; Nagai et al., 2005). Two-way ANOVA revealed significant differences in striatal $c d 68$ mRNA expression between treatment groups, time, and their interaction $(p<$ 0.0001 for treatment, time, and their interaction). Post hoc Bonferroni's analyses revealed that METH (5 $\mathrm{mg} / \mathrm{kg} \times 4$ ) caused a significant increase in striatal $c d 68$ expression at 3, 6, 12 and $24 \mathrm{~h}$ post-treatment as compared to saline alone $(t=3.15, p<0.05, t=4.37, p<0.001, t=8.58, p<0.001$ and $t=13.06, p<$ 0.001, respectively) (Fig. 7.2.). 


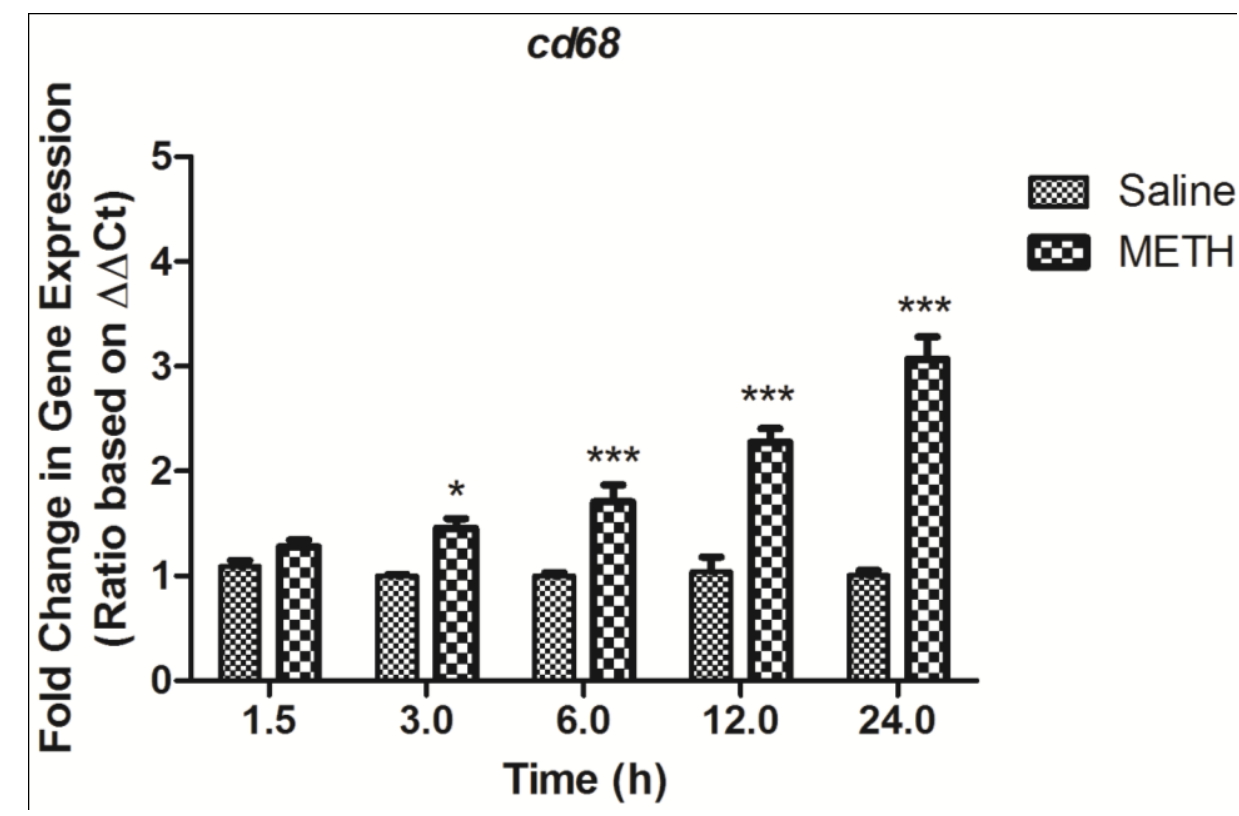

Figure 7.2. Methamphetamine treatment (METH, $5 \mathrm{mg} / \mathrm{kg} \times 4)$ time-dependently increased cd68 mRNA expression in the striatum, indicative of microglial cell activation. Two-way ANOVA, followed by Bonferroni's post hoc analysis; ${ }^{*} p<0.05, * * * p<0.001$, Saline vs. METH.

At $24 \mathrm{~h}$ post-treatment, a time point when the highest METH effects were observed, significant differences between treatment groups in cd68 mRNA expression were found (one-way ANOVA; F[3,39] $=37.84, \mathrm{p}<0.0001)$. Post hoc analysis confirmed that similar to the time course study, METH $(5 \mathrm{mg} / \mathrm{kg} \mathrm{x}$ 4) treatment resulted in a significant increase in striatal $c d 68$ expression as compared to saline alone (q $=13.63, \mathrm{p}<0.001)$. This METH-induced change in cd68 expression was significantly attenuated by SN79 pretreatment $(3 \mathrm{mg} / \mathrm{kg} \times 4)(q=9.11, \mathrm{p}<0.001)$, though the blockade was incomplete since SN79 pretreatment prior to METH still resulted in an elevated expression of $c d 68$ as compared to salinetreated controls $(q=4.51, p<0.05)$. In the absence of METH, SN79 treatment alone resulted in no significant changes in striatal cd68 mRNA expression (n.s.) (Fig. 7.3.A.).

To further confirm that SN79 mitigated processes related to METH-induced microglial activation, protein expression levels were determined for both CD68 and the commonly used microglial marker IBA-1 using immunohistochemistry at a later time point of $72 \mathrm{~h}$ post-treatment. One-way ANOVA revealed significant differences between treatment groups in CD68 protein expression at $72 \mathrm{~h}(\mathrm{~F}[3,19]=$ 
24.17, $\mathrm{p}<0.0001)$. Tukey's post hoc analysis revealed that METH treatment significantly increased striatal CD68 protein expression as compared to saline $(q=9.70, p<0.001)$. In accordance with the mRNA expression studies, SN79 pretreatment attenuated the striatal CD68 protein increases elicited by METH ( $q=9.67, p<0.001$ ), while having no effect on its own (n.s.) (Fig. 7.3.B.).

A

cd68$$
\text { 등 }
$$

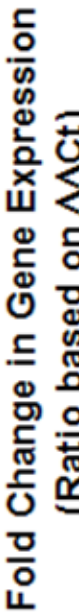

B
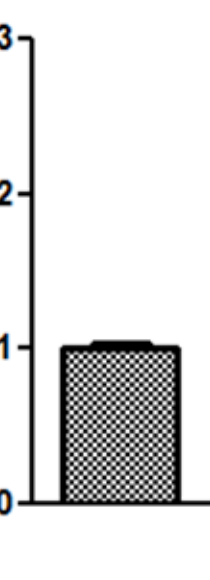

更

\begin{abstract}
Treatment
\end{abstract}
CD68

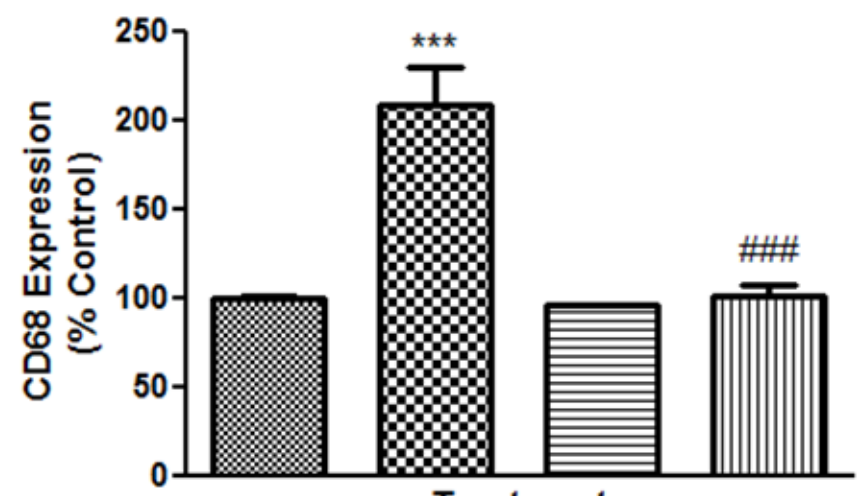

Treatment

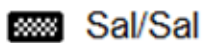

$\infty \mathrm{Sal} / \mathrm{METH}$

回 SN79/Sal

ㄸm SN79/METH
@al/Sal

- $\mathrm{Sal} / \mathrm{METH}$

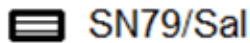

in. SN79/METH 


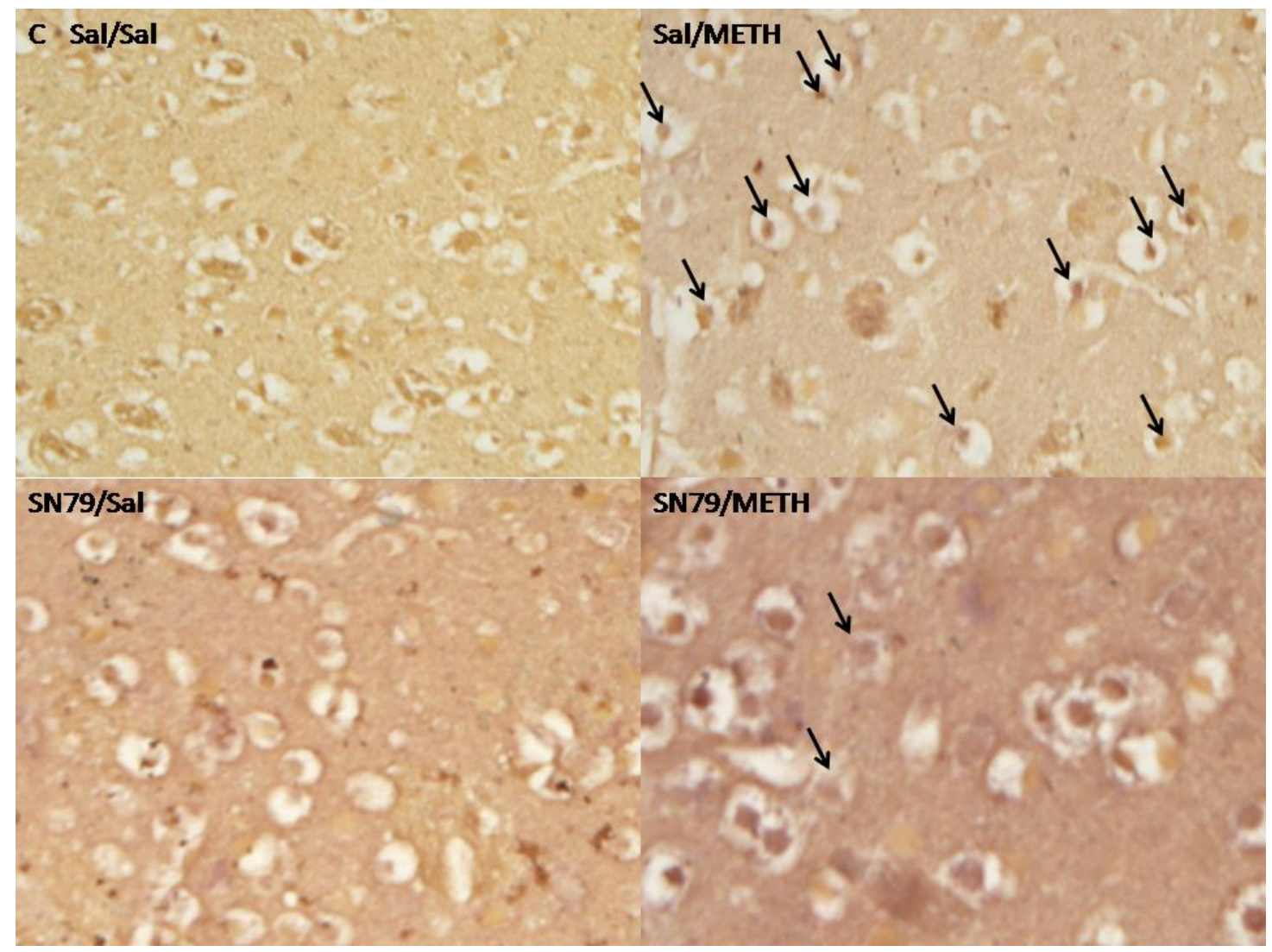

Figure 7.3. SN79 ( $3 \mathrm{mg} / \mathrm{kg} \times 4)$ pretreatment attenuated methamphetamine (METH, $5 \mathrm{mg} / \mathrm{kg} \times 4)$ induced increases in striatal CD68 mRNA and protein expression, indicative of a reduction in microglial activation. (A) SN79 treatment resulted in the blockade of METH-induced cd68 expression in the striatum $24 \mathrm{~h}$ post treatment. (B) SN79 pretreatment also blocked protein level increases in CD68 elicited by METH treatment at $72 \mathrm{~h}$. (C) Representative images of CD68 expression for each respective group. Black arrows depict microglia expressing high levels of CD68. 20X magnification. [One-way ANOVA, followed by post hoc Tukey's multiple comparison tests; ${ }^{*} p<0.05, * * * p<0.001$, Sal/Sal vs. respective group; \#\#\# p<0.001, Sal/METH vs. SN79/METH] Sal = saline.

Similar to the pattern of effects observed for CD68 protein, SN79 pretreatment also blocked METH-induced increases in striatal IBA-1 protein expression, providing confirmation of a reduction in another marker of microglial activation. One-way ANOVA revealed significant differences between groups in IBA-1 expression $(F[3,19]=150.00, p<0.0001)$. Post hoc analyses showed that METH treatment increased IBA-1 expression as compared to saline-treated controls at $72 \mathrm{~h}$ ( $\mathrm{q}=26.35, \mathrm{p}<$ 0.001), which was blocked by SN79 pretreatment $(q=24.40, p<0.001)$. Interestingly, SN79 alone 
resulted in a slight, yet statistically significant, paradoxical increase in IBA-1 expression compared to saline-treated controls $(q=4.41, p<0.05)$ (Fig. 7.4.).

\section{A}

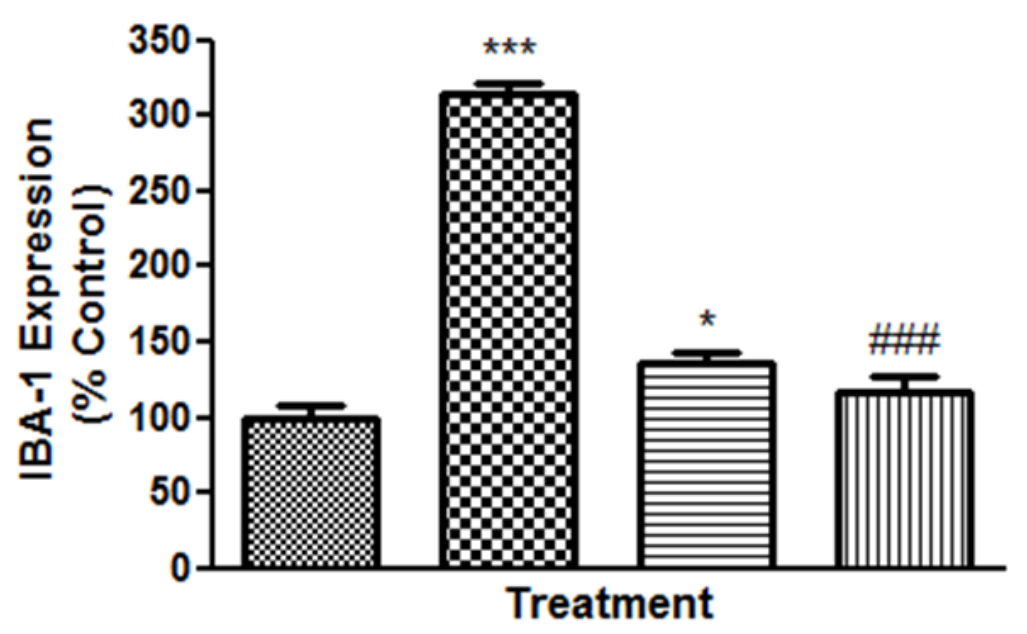

m Sal/Sal

m Sal/METH

曰SN79/Sal

IIII SN79/METH

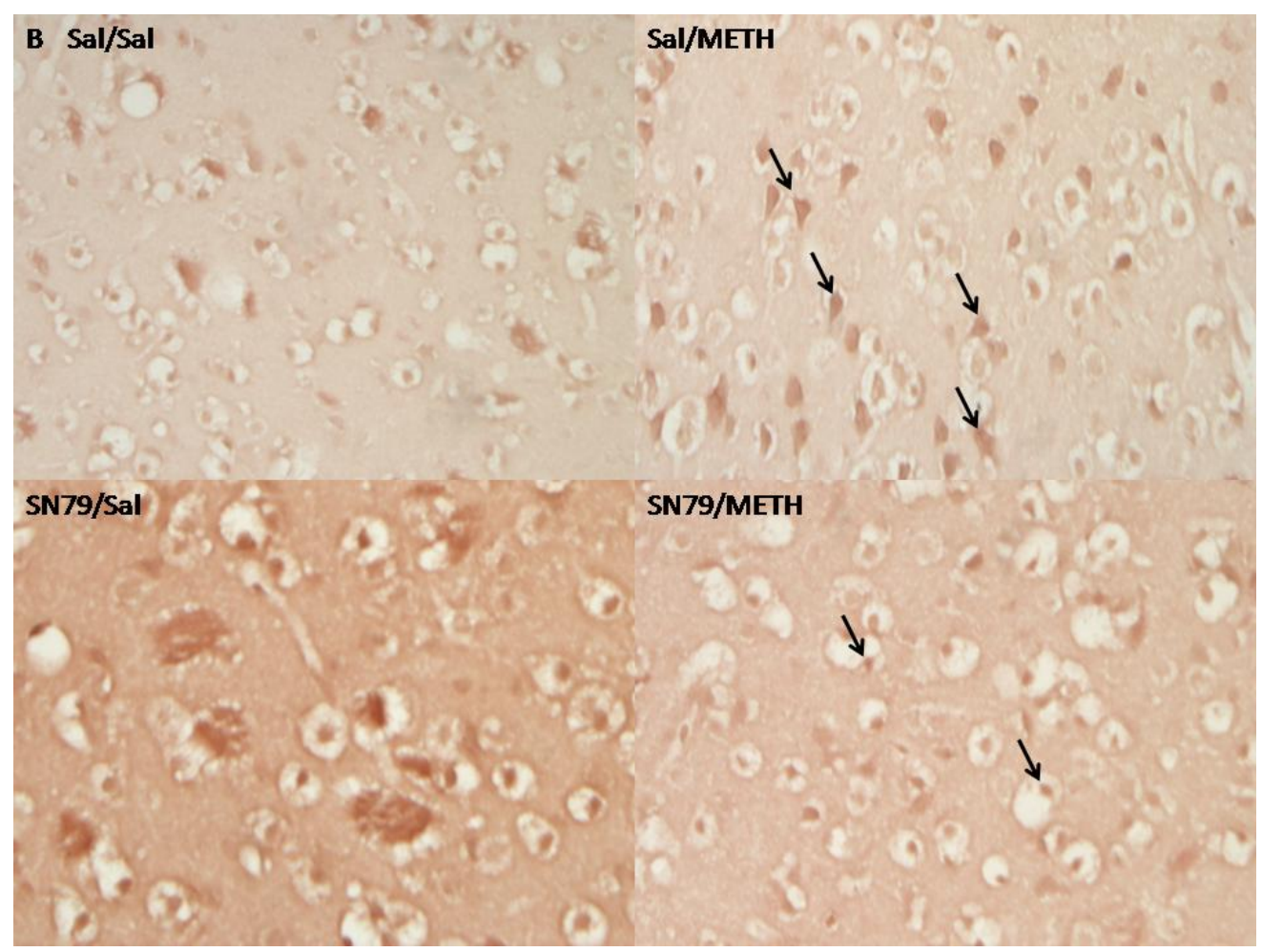

Figure 7.4. SN79 ( $3 \mathrm{mg} / \mathrm{kg} \times 4$ ) blocked methamphetamine (METH, $5 \mathrm{mg} / \mathrm{kg} \times 4$ )-induced increases in the microglial marker IBA-1 $72 \mathrm{~h}$ post treatment, confirming an attenuation of METH-induced microglial activation. (A) SN79 treatment blocked METH-induced increases in striatal IBA-1 expression at $72 \mathrm{~h}$. (B) Representative images of striatal IBA-1 expression for each respective group. Black arrows represent 
microglia with high levels of IBA-1 expression. 20X magnification. [One-way ANOVA, followed by post hoc Tukey's analysis; ${ }^{*} p<0.05,{ }^{* * *} p<0.001$, Sal/Sal vs. respective group; \#\#\# $p<0.001$, Sal/METH vs. SN79/METH] Sal = saline.

Immunohistochemical studies to determine the type of microglia (M1 or M2) that were being activated by METH within the striatum revealed a strong, preferential effect on M1 microglia. While METH treatment resulted in a significant increase in M1-type microglia (denoted by solely expressing CD68; as shown in Fig. 7.3.B.), it did not increase nor decrease levels of M2-type microglia in the striatum (denoted by co-expression of CD68 and CD163; one-way ANOVA, n.s.). Additionally, SN79 treatment had no effect on M2-type microglia in the striatum, either alone or in the presence of METH (n.s.) (Fig. 7.5.).

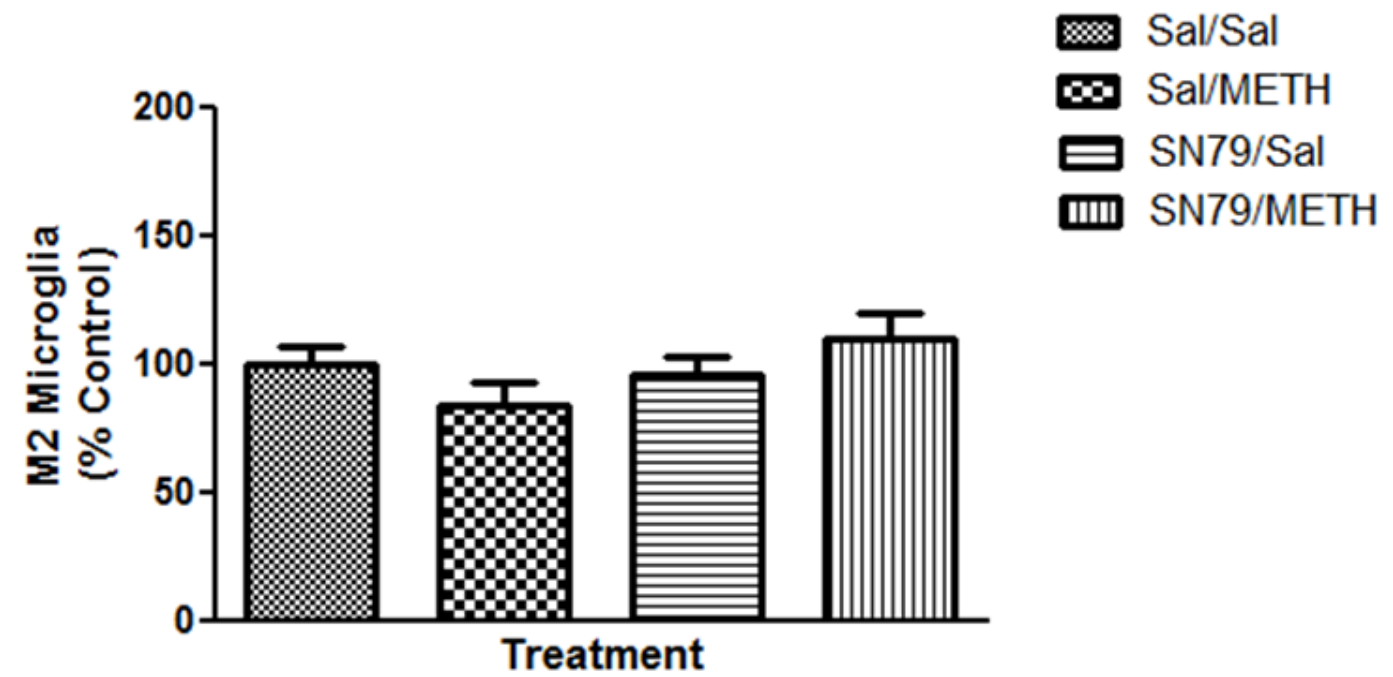

Figure 7.5. None of the drug treatments had a significant effect on levels of M2-type microglia present in the striatum $72 \mathrm{~h}$ post treatment (n.s.). Sal = saline, $\mathrm{METH}=$ methamphetamine $(5 \mathrm{mg} / \mathrm{kg} \times 4)$, SN79= SN79 (3 mg/kg x 4).

\subsubsection{IL-6 family inflammatory cytokine mRNA expression}

Along with the activation of pro-inflammatory M1 microglia, METH increased the mRNA expression of il-6, osm and lif, three IL-6 family cytokines within the striatum in a time-dependent 
manner. Two-way ANOVA revealed differences in il-6 expression between treatment groups $(\mathrm{p}<$ 0.0001). Post hoc analyses revealed that METH significantly increased il-6 mRNA expression at 1.5, 3, 6, 12 and $24 \mathrm{~h}(\mathrm{t}=3.69, \mathrm{p}<0.01, \mathrm{t}=5.87, \mathrm{p}<0.001, \mathrm{t}=4.94, \mathrm{p}<0.001, \mathrm{t}=6.25, \mathrm{p}<0.001$ and $\mathrm{t}=3.09, \mathrm{p}<$ 0.05, respectively) (Fig. 7.6.A.).

Two-way ANOVA also revealed significant differences between treatment groups, time, and their interaction in striatal osm mRNA expression ( $p<0.0001, p<0.05$ and $p<0.05$, respectively). Post hoc tests revealed that METH significantly increased osm expression as compared to saline treatment at $1.5,3,6$ and 12 h post- treatment $(t=5.68, p<0.001, t=6.41, p<0.001, t=5.86, p<0.001$ and $t=5.4$, $\mathrm{p}<0.001$, respectively) (Fig. 7.6.B.).

A significant difference was also found in striatal lif expression as a result of METH treatment, with two-way ANOVA showing a significant difference between treatment groups, time, and their interaction $(p<0.0001, p<0.01$ and $p<0.01$, respectively). Post hoc analyses confirmed that METH treatment significantly increased lif expression in the striatum at $1.5,3,6$, and $12 \mathrm{~h}$ post treatment as compared to saline $(t=6.02, p<0.001, t=7.36, p<0.001, t=4.73, p<0.001$ and $t=3.11, p<0.05$, respectively) (Fig. 7.6.C.). 

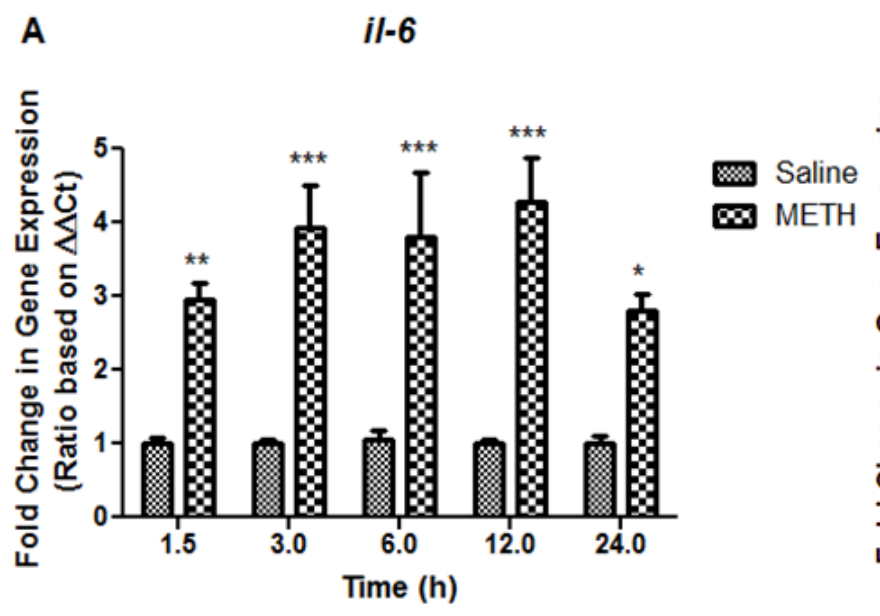

B

osm

C

lif

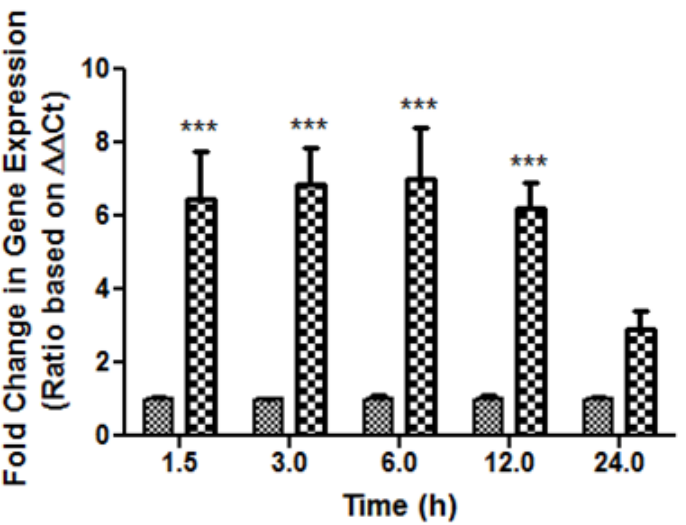

Saline

$\infty \mathrm{METH}$

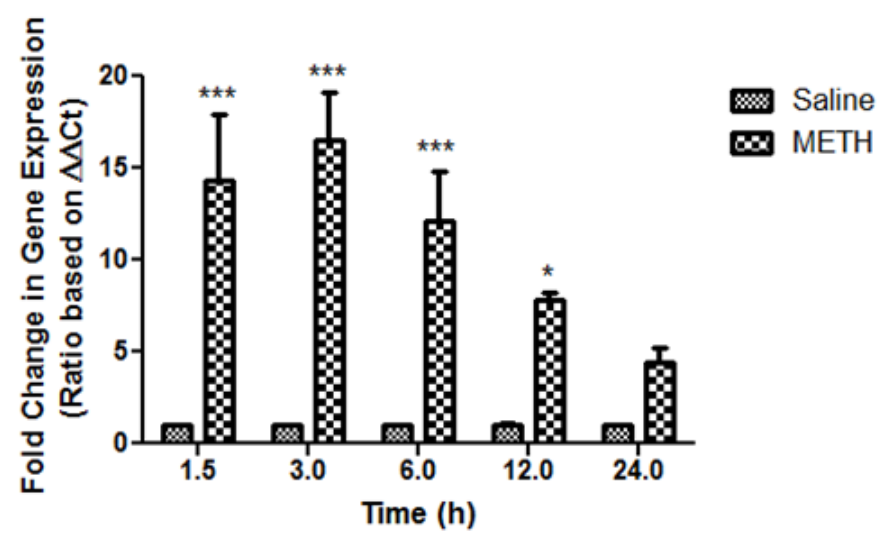

Figure 7.6. Methamphetamine treatment (METH, $5 \mathrm{mg} / \mathrm{kg} \times 4$ ) increased mRNA expression of interleukin-6 (IL-6) family cytokines in the striatum. (A) METH treatment resulted in significant increases in il-6 expression at all time points tested. (B) METH also increased striatal osm expression at 1.5, 3, 6 and $12 \mathrm{~h}$ post treatment. (C) METH further increased lif expression in the striatum at 1.5, 3, 6 and $12 \mathrm{~h}$ post treatment. Two-way ANOVA, followed by post hoc Bonferroni's analysis; * $p<0.05, * * p<0.01, * * *$ $p<0.001$, Saline vs. METH.

At $6 \mathrm{~h}$ post-treatment, a time point at which all three IL- 6 family cytokines studied were significantly up-regulated by METH in the time course studies described above, pretreatment with SN79 blocked these effects. One-way ANOVA revealed significant differences in striatal il-6 mRNA expression between the treatment groups at $6 \mathrm{~h}(\mathrm{~F}[3,38]=21.16, \mathrm{p}<0.0001)$. Similar to the time course experiments described above, METH treatment increased striatal il-6 expression as compared to salinetreated controls $(q=10.00, p<0.001)$, an effect mitigated by SN79 pretreatment $(q=6.38, p<0.001)$. 
SN79 treatment alone was also found to have no effect on striatal il-6 mRNA expression (n.s.) (Fig.

7.7.A.).
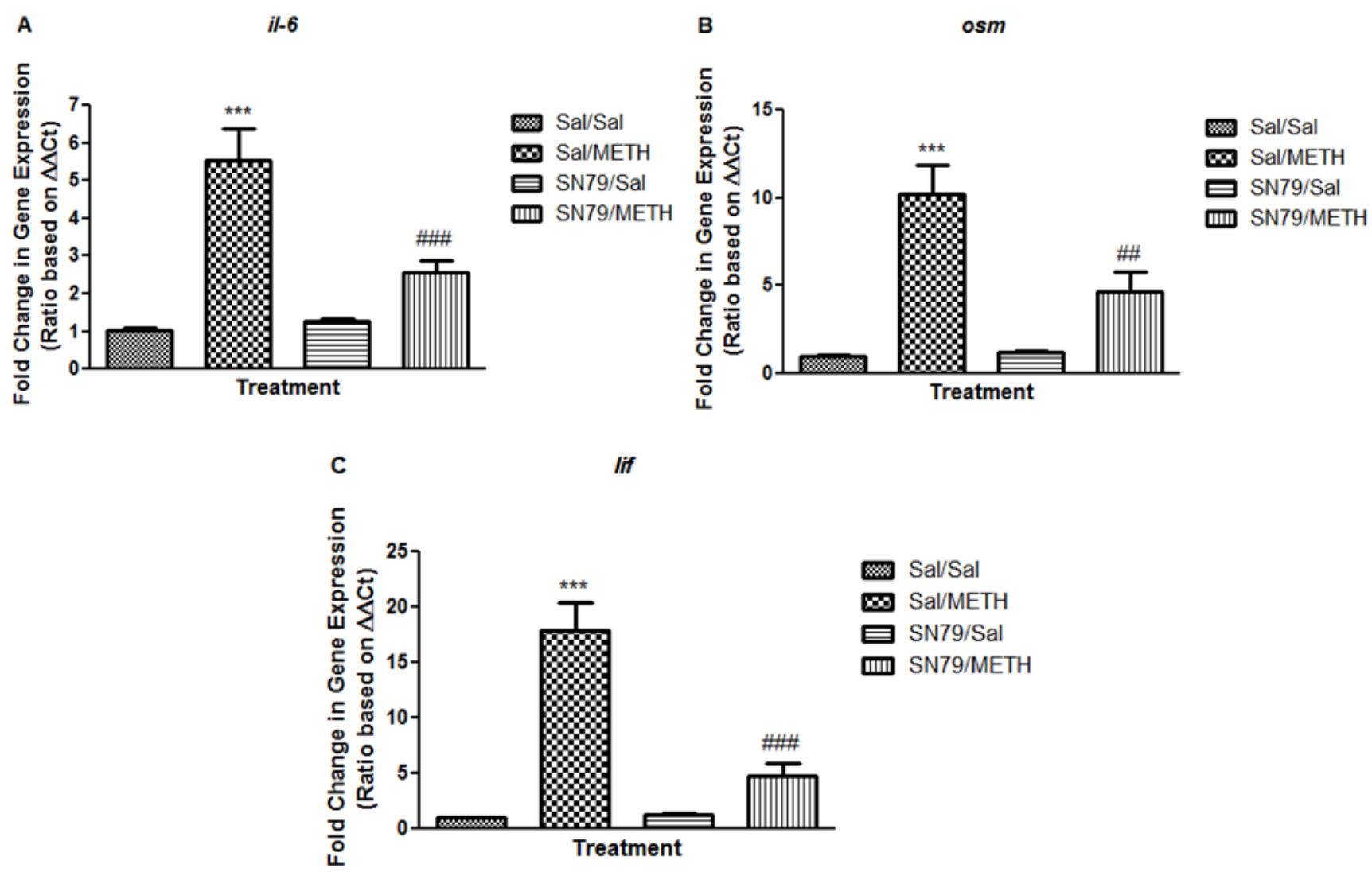

Figure 7.7. SN79 pretreatment ( $3 \mathrm{mg} / \mathrm{kg} \times 4$ ) blocked methamphetamine (METH, $5 \mathrm{mg} / \mathrm{kg} \times 4$ )-induced increases in mRNA expression of IL-6 family cytokines in the striatum $6 \mathrm{~h}$ post treatment. (A) SN79 attenuated METH-induced increases in il-6 expression. (B) METH treatment significantly increased striatal osm expression, an effect mitigated by SN79 treatment. (C) SN79 attenuated METH-induced increases in lif expression. One-way ANOVA, followed by post hoc Tukey's multiple comparison tests; $* * *$ p $<0.001$, Sal/Sal vs. Sal/METH; \#\# p<0.01, \#\#\# p<0.001, Sal/METH vs. SN79/METH. Sal = saline.

Pretreatment with SN79 also mitigated METH-induced increases in osm expression in the striatum. One-way ANOVA showed significant differences in osm expression between treatment groups $(F[3,38]=19.48, p<0.0001)$. Tukey's post hoc analysis revealed that METH significantly increased osm mRNA expression as compared to saline controls $(q=9.45, p<0.001)$. SN79 treatment attenuated METH-induced increases in osm expression ( $q=5.57, p<0.001)$, while having no effects on its own compared to saline-treated controls (n.s.) (Fig. 7.7.B.). 
Additionally, SN79 attenuated METH-induced increases in lif expression in the striatum. Oneway ANOVA showed a difference between the treatment groups in striatal lif expression $(F[3,38]=$ 31.89, $\mathrm{p}<0.0001$ ), and post hoc analysis revealed that METH caused a significant increase in lif as compared to saline controls $(q=11.99, p<0.001)$. SN79 blocked METH-induced increases in lif mRNA expression ( $q=9.10, p<0.001$ ), while having no effects on its own (n.s.) (Fig. 7.7.C.).

\subsection{Discussion}

Data from the current study provides evidence that sigma receptor antagonists, such as SN79, block METH-induced microglial activation. SN79 blocked METH-induced increases in mRNA and protein of the microglial markers CD68 and IBA-1. Additionally, SN79 treatment blocked METH-induced increases in the expression of pro-inflammatory IL-6 family cytokines in the striatum. The ability of SN79 to block METH-induced microglial activation and increases in the expression of pro-inflammatory cytokines provides evidence that this may be a mechanism by which sigma receptor antagonists mitigate the neurotoxic effects of METH.

The characterization of microglial activation as a result of METH exposure in preclinical rodent models has provided evidence that microglial activation is associated with METH-induced neurotoxicity (Kelly et al., 2012; LaVoie et al., 2004; Thomas and Kuhn, 2005; Thomas et al., 2004c). METH activates microglia in a dose- and time-dependent manner coinciding with reductions in striatal dopamine levels elicited by exposure to the drug (Thomas et al., 2004c). Amphetamine-type drugs that do not cause neurotoxicity such as L-methamphetamine and fenfluramine, fail to activate microglia, providing further evidence that microglial activation is associated with neurotoxicity (Thomas et al., 2004a). Additionally, environmental manipulations that block METH-induced dopaminergic neurotoxicity such as reductions in ambient temperature also block METH-induced microglia activation (Thomas et al., 2004c). This same study, however, also ruled out hyperthermia as a causative factor in microglia activation as 
hyperthermia elicited by simple ambient temperature increases or pharmacologic treatments other than METH were unable to cause microglial activation (Thomas et al., 2004c). These results thus imply that hyperthermia plays a significant contributory, but not causative, role in microglia activation by METH, and compounds such as SN79 may convey beneficial effects at least in part through the blockade of METH-induced hyperthermia.

Increases in pro-inflammatory cytokines in the striatum following a neurotoxic regimen of METH have been shown previously (Kelly et al., 2012; Kuhn et al., 2006). Recently, increases in striatal expression levels of IL-6 family pro-inflammatory cytokines such as OSM, LIF and IL-6 by METH exposure were reported (Kelly et al., 2012). Further support of a role for IL-6 and its family members in the neurotoxic effects of METH are studies showing a reduction in dopaminergic and serotonergic neurtoxicity by METH in IL-6 knockout mice (Ladenheim et al., 2000). These effects were found independently of any alterations in METH-induced hyperthermia between knockout animals and wild type controls, indicative of a specific effect of IL-6 reduction on neurotoxicity (Ladenheim et al., 2000). The activation of microglia with a concomitant increase in pro-inflammatory cytokines such as IL-6 is believed to result in neuronal toxicity through excitotoxicity, apoptosis, immune activation, and generation of ROS/RNS, all of which are hypothesized to contribute to the neurotoxic effects of METH (Cadet et al., 2003; Cadet and Krasnova, 2009; Smith et al., 2012b). Additionally, the extent of dopaminergic nerve terminal damage has recently been found to be linked to microglial activation, further inferring that microglial activation and neuroinflammation may play a prominent role in the ability of METH to elicit neurotoxic reponses (Kelly et al., 2012; Thomas and Kuhn, 2005).

The ability of sigma receptor antagonists such as SN79 to block microglial activation and increases in IL- 6 family cytokine mRNA elicited by METH may be one mechanism by which they are able to mitigate the neurotoxic effects of METH. Sigma receptors themselves are known to be expressed in microglia and ligands targeting sigma receptors have been shown to modulate microglia function and 
activation in in vitro and in vivo models (Ajmo et al., 2006; Hall et al., 2009; Ruscher et al., 2012). The precise mechanism by which this occurs is currently unknown; however, sigma receptor modulation alters intracellular $\mathrm{Ca}^{2+}$ levels that have been associated with microglial activation and migration in vitro (Cuevas et al., 2011; Hall et al., 2009). This effect does not appear to be subtype specific, as ligands targeting sigma-1 and sigma-2 receptors independently have been shown to modulate microglial responses to a variety of microglial activators such as ATP, uridine triphosphate (UTP), LPS and monocyte chemotactant protein-1 (Cuevas et al., 2011; Hall et al., 2009; Yao et al., 2010). The ability of sigma receptor ligands to alter intracellular $\mathrm{Ca}^{2+}$ levels within microglia, thereby altering activation and migration, may result in the attenuation of the production of pro-inflammatory cytokines in activated microglia and the subsequent neurotoxicity associated with METH treatment (Cuevas et al., 2011). Interestingly, METH-induced microglial activation has also been linked to behavioral effects associated with usage of the drug in addition to neurotoxicity. Minocycline is an anti-inflammatory agent known to affect microglia that has been shown in rodent models to block METH-induced increases in locomotor activity, development of behavioral sensitization, impairments in recognition memory and rewarding effects of the drug as assessed by a conditioned place preference paradigm (Hashimoto et al., 2007b; Mizoguchi et al., 2008; Zhang et al., 2006). A recent case report has also provided clinical evidence that minocycline treatment was linked to a reduction in METH-induced psychosis in a patient experiencing hallucinations associated with chronic METH usage (Tanibuchi et al., 2010). These reports provide evidence of a link between microglial activation and the behavioral and neurotoxic effects of METH. Notably, sigma receptor antagonists have been shown in rodent models to block METH-induced increases in locomotor activity, development and expression of behavioral sensitization and impairments in recognition memory, in addition to blocking hyperthermia and neurotoxicity associated with the drug (Kaushal et al., 2012b; Kaushal et al., 2011f; Seminerio et al., 2012a; Seminerio et al., 2011). The current report however, is the first showing that sigma receptor antagonists are able to 
mitigate METH-induced microglial activation. It remains to be seen if the ability of sigma receptor antagonists to mitigate the aforementioned METH-induced behaviors is associated with modulation of microglial activity.

It has recently been hypothesized that compounds targeting glial cells may be effective clinical treatments for the abuse of psychostimulants, including METH (Cooper et al., 2012). Given the current lack of treatments aimed at reducing METH usage or the negative effects associated with chronic METH usage, the further study of compounds that modulate glial cell function is certainly warranted. Compounds such as SN79 may be promising candidates to treat the negative complications of psychostimulant abuse by acting through a variety of mechanisms including microglial activation. SN79 has previously been shown to display pharmacokinetic parameters amenable to clinical usage, and is orally bioavailable (Kaushal et al., 2011e), making it a potentially viable compound for further drug development. Future studies aimed at further delineating the mechanisms by which sigma receptor antagonists mitigate the neurotoxic actions of METH and further exploring drug development of these compounds for clinical usage are warranted.

\subsection{Contributions}

Matthew Robson conducted all in vivo studies, quantitative real-time PCR and analyzed all data. Immunohistochemistry was conducted by Ryan Turner and Zachary Naser and data was analyzed by Matthew Robson.

\section{This chapter has previously been published as:}

Robson MJ, Turner RC, Naser ZJ, McCurdy CR, Huber JD, Matsumoto RR. SN79, a sigma receptor ligand, blocks methamphetamine-induced microglial activation and cytokine upregulation. Experimental Neurology. 2013. Accepted In Press. PubMed ID: 23631864 


\title{
Chapter 8
}

SN79, a sigma receptor ligand, attenuates methamphetamine-induced astrogliosis through a blockade of OSMR/gp130 signaling and STAT3

\author{
phosphorylation
}




\subsection{Introduction}

Methamphetamine (METH) is an illicit substance that acts as an addictive psychostimulant by modulating monoamine signaling within the central nervous system (CNS). Chronic METH abuse results in several negative side effects, including dopaminergic nerve terminal toxicity (Romanelli and Smith, 2006; Volkow et al., 2001a; Volkow et al., 2001b), altered morphology in the substantia nigra (Todd et al., 2013), and an increased risk of developing Parkinson's disease later in life (Callaghan et al., 2010; Callaghan et al., 2012).

In addition to having dramatic effects on dopaminergic neurons, METH affects a variety of other cell types located within the CNS (Cadet and Krasnova, 2009), including astrocytes in regions of the brain affected by its neurotoxic actions (Bowyer et al., 1994; O'Callaghan and Miller, 1994; Pu et al., 1994). Astrocytes isolated from regions of the brain affected by the toxic effects of METH appear more sensitive to the effects of the drug than comparable cell populations from other areas (Lau et al., 2000; Stadlin et al., 1998). In vitro studies using isolated astrocytes have confirmed that METH can directly exert actions on these cells; however, it is currently unclear whether in vivo activation of astrocytes by METH also results from direct actions on this cell type or whether it is a consequence of neuronal damage and neuroinflammation (Hebert and O'Callaghan, 2000; Kelly et al., 2012; Lau et al., 2000; Narita et al., 2006; Sriram et al., 2004; Stadlin et al., 1998).

Astrocytes are activated in response to a variety of CNS insults through a process termed astrogliosis whereby they undergo distinct morphological changes and display an increase in the expression of glial fibrillary acidic protein (GFAP) (Raivich et al., 1999). One mechanism by which astrocytes can be activated is through the induction of STAT3 phosphorylation through JAK/STAT signaling events (Hebert and O'Callaghan, 2000). It is hypothesized that this phosphorylation occurs through gp130-mediated cytokine signaling events initiated by inflammatory processes (Hebert and O'Callaghan, 2000; Van Wagoner and Benveniste, 1999). The phosphorylation and therefore activation 
of STAT3 in astrocytes can be mediated through oncostatin M (OSM)-mediated signaling through the oncostatin M receptor (OSMR) (Van Wagoner et al., 2000). OSMR is an IL-6-type cytokine receptor that dimerizes with gp130 and mediates intercellular signaling events, including STAT3 (Tyr-705) phosphorylation (Chen and Benveniste, 2004; Van Wagoner et al., 2000). Interestingly, OSM signaling through OSMRß/gp130 is believed to modulate astrocyte function and the expression of GFAP is decreased in mice deficient in gp130 (Chen et al., 2006; Nakashima et al., 1999), providing evidence that signaling through OSMRß/gp130 complexes is involved in GFAP upregulation and subsequent astrogliosis. Furthermore, METH results in increased expression of osmr and gfap in regions of the brain affected by the neurotoxic effects of the drug in rodents (Thomas et al., 2004b). There is, however, a paucity of studies confirming the effect of METH on the transcriptional regulation of osmr in astrocytes per se, although a recent report has shown that osmr expression increases in astrocytes activated in vivo by other insults, such as ischemic stroke or peripheral lipopolysaccharide (LPS) injections (Zamanian et al., 2012).

Exacerbating the problem of METH-induced neurotoxicity is the current lack of FDA approved pharmacotherapies for treating the negative health effects of METH usage. One potentially promising molecular target for the production of medications aimed at counteracting these effects are sigma receptors. There are currently two known subtypes of sigma receptors (Hellewell and Bowen, 1990). Sigma-1 receptors are ligand-activated chaperones that modulate various intracellular signaling cascades making them particularly interesting targets for the development of potential pharmacotherapies for the treatment of drug abuse (Matsumoto, 2009; Robson et al., 2012). METH interacts with both subtypes of sigma receptors, denoted sigma-1 and sigma-2 receptors, at physiologically relevant concentrations and sigma receptor antagonists have been shown to mitigate the neurotoxic effects of METH on dopaminergic and serotonergic systems within the CNS (Kaushal et al., 2012b; Matsumoto et al., 2008; Nguyen et al., 2005). Sigma receptors are expressed in astrocytes and 
sigma receptor modulation has been shown to modulate the activity of astrocytes both in vitro and in vivo (Ajmo et al., 2006; Klouz et al., 2003); however whether sigma receptor modulation alters METHinduced astrocyte activation has yet to be determined.

Therefore, the primary purpose of the current study was to determine if a prototypic sigma receptor antagonist mitigates $\mathrm{METH}$-induced reactive astrogliosis and neuronal degeneration. Secondly, we wanted to determine if sigma receptor modulation results in a blockade of OSMR $\beta /$ gp130-induced STAT3 phosphorylation which has been implicated in the activation of astrocytes following CNS insults. Herein, we provide evidence that METH treatment results in an increase in OSMR expression and signaling through STAT3 in astroctyes with subsequent reactive astrogliosis, effects mitigated by treatment with the sigma receptor antagonist and potential drug development candidate, SN79 (6acetyl-3-(4-(4-(4-fluorophenyl)piperazin-1-yl)butyl)benzo[d]oxazol-2(3H)-one).

\subsection{Materials and Methods}

\subsubsection{Drugs and chemicals}

(+)-Methamphetamine hydrochloride was obtained from Sigma Aldrich (St. Louis, MO). SN79 was synthesized as previously described (Kaushal et al., 2011e) and provided by Dr. Christopher R. McCurdy (University of Mississippi, University, MS). All drugs administered to animals were dissolved in sterile saline solution ( $0.1 \mathrm{ml} / 10 \mathrm{~g}$ body weight) (Teknova, Fisher Scientific, Pittsburgh, PA). All other chemicals were obtained from Sigma Aldrich (St. Louis, MO) unless otherwise specified.

\subsubsection{Animals}

Male, Swiss Webster mice (24-28 g; Harlan, Indianapolis, IN) were utilized for all experiments. Mice were housed in groups of five, on a 12:12 h light/dark cycle with food and water ad libitum. Mice 
were randomly assigned to their respective treatment groups. All experiments were performed as approved by the Animal Care and Use Committee at West Virginia University.

\subsubsection{Repeated dosing paradigm}

Mice were randomly distributed for each experiment into one of four treatment groups: 1 ) Saline/Saline (0.1 ml/10 g body weight), 2) Saline/METH 5 mg/kg, 3) SN79 3 mg/kg/Saline, or 4) SN79 3 $\mathrm{mg} / \mathrm{kg} / \mathrm{METH} 5 \mathrm{mg} / \mathrm{kg}$. The first compound listed in each pair (Saline or SN79) was administered intraperitoneally (i.p.) as a pretreatment $15 \mathrm{~min}$ prior to the second compound in each treatment group (Saline or METH, i.p.). Each animal underwent four pretreatments/treatments at $2 \mathrm{~h}$ intervals as previously described (Kaushal et al., 2012b).

The METH dose $(5 \mathrm{mg} / \mathrm{kg} \times 4$ ) was selected based upon previous dose response experiments, where it consistently produced significant dopaminergic deficits in the striatum in this specific model (Matsumoto et al., 2008; Seminerio et al., 2012a). The dose of SN79 (3 mg/kg x 4) was selected due to previously reported dose response experiments assessing the ability of this compound to mitigate the striatal dopaminergic deficits elicited by METH treatment (Kaushal et al., 2012b).

\subsubsection{Quantitative real time PCR}

At various timepoints post-treatment (as measured from the last injection), bilateral striatum samples were collected, flash frozen in liquid nitrogen and stored at $-80^{\circ} \mathrm{C}$ for later use. Total RNA was then extracted from flash frozen striatum samples using Trizol reagent (Invitrogen, Grand Island, NY) according to manufacturer's instructions. The total RNA concentration for each sample was quantified by spectral absorption, and the purity of the sample checked to confirm that the $260 / 280$ ratio was in the range of 1.8-2.1. Samples of cDNA were prepared by reverse transcription using a high capacity reverse transcription kit (Applied Biosystems, Foster City, CA). Each sample reaction included 
MultiScribe TM Reverse Transcriptase and random primers, with thermal cycler conditions set as follows: step 1 at $25^{\circ} \mathrm{C}$ for $10 \mathrm{~min}$, step 2 at $37^{\circ} \mathrm{C}$ for $120 \mathrm{~min}$, step 3 at $85^{\circ} \mathrm{C}$ for $5 \mathrm{~s}$, and step 4 at $4^{\circ} \mathrm{C}$ for $10 \mathrm{~min}$.

For PCR amplification, TaqMan $^{\circledR}$ Universal PCR Master Mix and the following probes were obtained from Applied Biosystems (Foster City, CA): 18S (Hs99999901_s1) for use as an endogenous control gene, GFAP (Mm01253033_m1) and OSMR (Mm00495424_m1). The reaction mixture was prepared according to the manufacturer's instructions, with the following thermal cycling conditions: initial holding at $50^{\circ} \mathrm{C}$ for 2 min required for optimal AmpErase ${ }^{\circledR}$ UNG activity, followed by a first denaturing step at $95^{\circ} \mathrm{C}$ for $10 \mathrm{~min}$, then 45 cycles at $95^{\circ} \mathrm{C}$ for $15 \mathrm{~s}$, and at $60^{\circ} \mathrm{C}$ for $1 \mathrm{~min}$. Data from real-time PCR measurements were calculated using the $\Delta \Delta \mathrm{Ct}$ method. The threshold value was set at 0.2 and the threshold cycle (Ct value) of each gene was then normalized to 18s rRNA.

\subsubsection{Immunohistochemistry}

At $72 \mathrm{~h}$ post-treatment (after the last injection), animals were anesthetized and perfused transcardially with phosphate buffered saline (PBS) followed by $4 \%$ paraformaldehyde. The entire brain was removed and immediately placed in $4 \%$ paraformaldehyde for $24 \mathrm{~h}$. Following fixation, the brains were processed using a Tissue-Tek VIP 5 automatic tissue processor (Sakura Finetek, Torrance, CA) and then embedded in paraffin with a Tissue-Tek TEC 5 embedding console system (Sakura Finetek).

Embedded tissues were sliced in $6 \mu \mathrm{m}$ sections using a Leica RM2235 microtome (Leica Microsystems, Buffalo Grove, IL), and slices mounted on glass slides for staining. All slides were heat-fixed and deparaffinized via a series of xylene and alcohol washes prior to immunohistochemical procedures. Following deparaffinization and rehydration, endogenous peroxidases were quenched using a $10 \%$ methanol and 10\% hydrogen peroxide solution in PBS for $30 \mathrm{~min}$. Slides were permeabilized for $1 \mathrm{~h}$ using a $1.8 \%$ L-lysine, $5 \%$ horse serum, and $0.1 \%$ Triton X-100 in Dulbecco's phosphate buffered saline 
(DPBS). Sections were then incubated in polyclonal antibody raised in rabbit against anti-cow GFAP (DAKO, Glostrup, Denmark) at a dilution of 1:500 in 5\% horse serum in PBS overnight at $4^{\circ} \mathrm{C}$. Next, sections were washed twice for 10 min each in PBS prior to application of Alexa Fluor 488 goat antirabbit IgG (Invitrogen, Grand Island, NY) at a dilution of 1:100 in PBS for $3 \mathrm{~h}$. Following the incubation in Alexa Fluor 488 goat anti-rabbit IgG, slides were rinsed twice for 10 min each in PBS. Then, the tissues were incubated in polyclonal antibody raised in goat anti-mouse OSMR (LifeSpan Biosciences, Seattle, WA) at a dilution of 1:200 in 5\% horse serum in PBS overnight at $4^{\circ} \mathrm{C}$. Following the incubation in primary antibody, the slides were rinsed twice for 10 min each in PBS prior to the application of biotinylated anti-goat IgG (Vector Laboratories, Burlingame, CA) at a dilution of 1:10,000 in $5 \%$ horse serum in PBS for $2 \mathrm{~h}$. Following the incubation in biotinylated anti-goat IgG, slides were rinsed twice for 10 min each in PBS prior to the application of Streptavidin Alexa Fluor 546 (Life Technology, Carlsbad, CA) at a dilution of 1:100 in PBS for $1 \mathrm{~h}$. Slides were rinsed in PBS for $10 \mathrm{~min}$ and then coverslipped with Vectashield Mounting Media with 4',6-diamidino-2-phenylindole (DAPI) (Vector Laboratories, Burlingame, CA). Finally, slides were sealed with acrylic and stored in the dark in a laboratory refrigerator at $4^{\circ} \mathrm{C}$. Images were acquired using a Zeiss Axio Imager 2 microscope (Oberkocken, Germany) and quantified using ImageJ software (NIH; http://rsbweb.nih.gov/ij/) by analyzing fluorescence intensity as compared to background using standard colocalization quantification techniques.

In addition to nerve terminal damage, METH has been reported to result in striatal apoptosis and cellular toxicity in vivo (Deng et al., 2001; Jayanthi et al., 2005). Fluoro-Jade staining is a viable marker of neuron degeneration as a result on amphetamine treatment in rodents (Eisch et al., 1998; Schmued and Hopkins, 2000); however whether sigma receptor ligands mitigate METH-induced striatal neuronal degeneration has yet to be determined. For Fluoro-Jade B staining, sections were incubated in $0.06 \%$ potassium permanganate for $10 \mathrm{~min}$, washed in deionized water, and incubated in $0.0004 \%$ 
Fluoro-Jade B in $0.1 \%$ acetic acid for 20 min. Sections were then washed, coverslipped, and sealed. For bright-field visualization of GFAP, slides were rinsed in DPBS, permeabilized with $1.8 \%$ L-lysine, $4 \%$ horse serum, and $0.2 \%$ Triton X-100 in DPBS. Sections were incubated overnight with a polyclonal antibody raised in rabbit against anti-cow GFAP at a dilution of 1:500 in $4 \%$ horse serum in PBS. Sections were washed and incubated for $4 \mathrm{~h}$ in biotinylated anti-rabbit IgG $(1: 10,000)$ in PBS with $4 \%$ horse serum. Diaminobenzidine (DAB) was used as a chromagen following incubation in avidin D-horseradish peroxidase (HRP) for $1 \mathrm{~h}$.

Bright-field immunohistochemistry was quantified using stereology and the optical fractionator technique as previously described (Schmitz and Hof, 2005; Smith et al., 2012a; Turner et al., 2012). Briefly, a region of interest encompassing the striatum was drawn at low power using an Olympus AX70 microscope and accompanying Stereolnvestigator software (MBF Bioscience, Williston, VT). Counting frames were randomly selected by the software and the cell-type of interest marked by an observer blinded to treatment. The volume of the region of interest previously identified was then determined by the software and the number of cells marked by the observer returned. Counting frames were $75 \mu \mathrm{m}$ on each side with a depth of $6 \mu \mathrm{m}$.

\subsubsection{Immunoblotting}

Twelve hours post-treatment (time from last treatment), animals were sacrificed utilizing focused microwave irradiation (Muromachi Microwave Applicator, TMW-4012C, $10 \mathrm{~kW}$ output) to preserve the phosphorylation states of proteins within each animal post-mortem, including STAT3 (Tyr705) (O'Callaghan and Sriram, 2004). Mice were briefly restrained in a water-jacketed holder and inserted into the machine head. The microwave beam was then activated ( $5 \mathrm{~kW}$ power setting for $1 \mathrm{~s}$ ) and animals were subsequently removed from the holder. Brains were then removed and bilateral striatum samples dissected, flash frozen and stored at $-80^{\circ} \mathrm{C}$. Protein was isolated from each respective 
sample by sonication in $25 \mu \mathrm{l}$ of hot $\left(85-95^{\circ} \mathrm{C}\right) 1 \%$ sodium docecyl sulfate (SDS) as previously described (O'Callaghan and Sriram, 2004). The protein concentration of each sample was measured using a Coomassie Plus Bradford Assay Kit (Pierce, Rockford, IL) which is based upon a modified form of the Bradford assay (Bradford, 1976). Samples were run using $20 \mu \mathrm{g}$ of protein/well using Tris- $\mathrm{HCl} 10 \%$ precast 15-well gels (Bio-Rad, Hercules, CA) in combination with 5X Lammeli sample buffer. Gels were run using a mini-PROTEAN system (Bio-Rad) and gels were equilibrated for $15 \mathrm{~min}$ in Towbin's buffer prior to transfer to polyvinylidene fluoride (PVDF) membranes (Bio-Rad) using semi-dry electrophoretic transfer cells (Bio-Rad). Membranes were blocked using 5\% fat-free milk/tris buffered saline and Tween 20 (TBST) for $2 \mathrm{~h}$ at room temperature. Membranes were incubated with primary phosphoSTAT3 (Tyr-705) or STAT3 antibody (Cell Signaling, Danvers, MA) at a concentration of 1:1,000 overnight at $4^{\circ} \mathrm{C}$. Anti-rabbit IgG HRP-linked antibody (Cell Signaling) was used as a secondary antibody at a concentration of 1:2000 with gentle shaking for $2 \mathrm{~h}$. An HRP-conjugated $\beta$-actin rabbit monoclonal antibody (Cell Signaling) was used as an endogenous control for all samples at a concentration of 1:10,000 and incubated for $1 \mathrm{~h}$. Molecular weight determination was conducted using a biotinylated protein ladder (Cell Signaling). Imaging was conducted using 20X LumiGLO chemiluminescent substrate (Cell Signaling) according to manufacturer's instructions. Images were converted to 8-bit and analyzed using densitometry with background subtraction and normalized to $\beta$-actin using ImageJ software (NIH; http://rsbweb.nih.gov/ij/).

\subsubsection{Statistical analysis}

Statistical analyses were conducted using either one-way or two-way analysis of variance (ANOVA), followed where applicable by post hoc Tukey's or Bonferroni's multiple comparison tests. Data was analyzed using GraphPad Prism 5 (GraphPad Software, La Jolla, CA). $p<0.05$ was considered statistically significant. 


\subsection{Results}

\subsubsection{METH time dependently increases striatal gfap mRNA expression}

Repeated treatment with METH (5 mg/kg x 4) resulted in significant increases in transcriptional upregulation of GFAP. As shown in Figure 8.1., two-way ANOVA revealed that METH caused timedependent increases in gfap mRNA expression ( $p<0.0001$ for time, treatment and their interaction). Bonferroni's post hoc tests revealed that METH treatment significantly increased gfap expression 12 and 24 h post-treatment $(t=3.97, p<0.01$ and $t=7.66, p<0.001$, respectively).

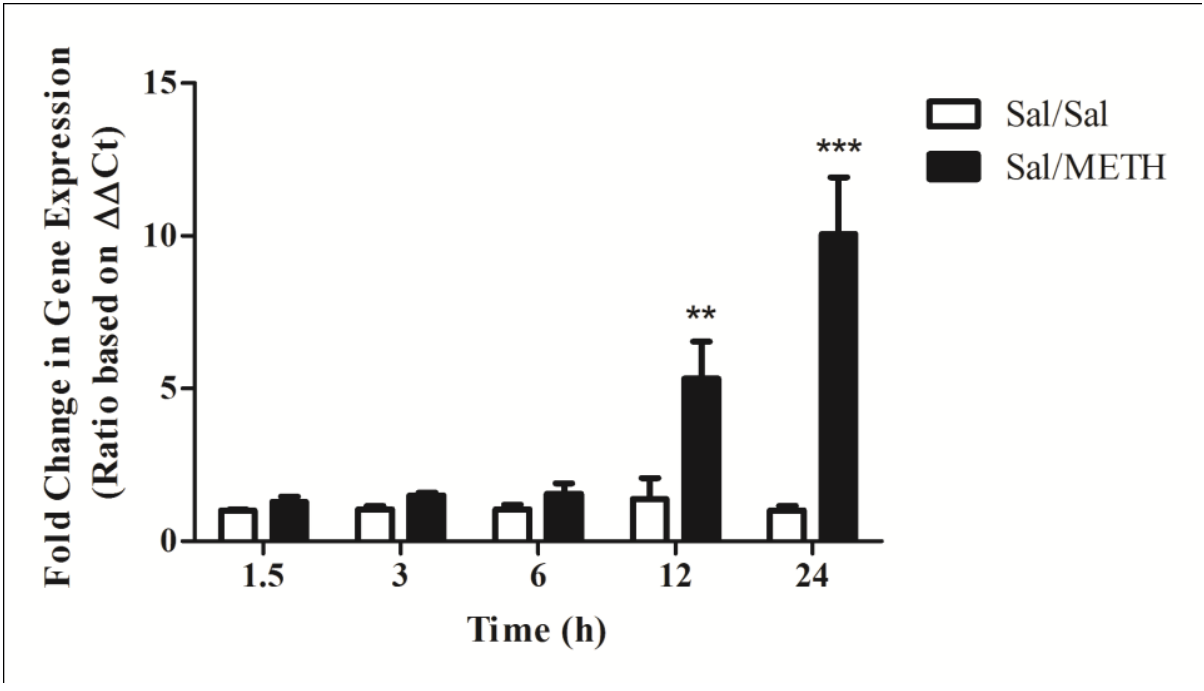

Figure 8.1. Methamphetamine (METH, $5 \mathrm{mg} / \mathrm{kg} \times 4$ ) treatment resulted in time-dependent increases in striatal gfap mRNA expression. METH was found to significantly increase gfap expression at both 12 and $24 \mathrm{~h}$ post-treatment. Two-way ANOVA, followed by post hoc Bonferroni's multiple comparison tests. $* * p<0.01, * * * p<0.001$, Sal/METH vs. Sal/Sal. Sal = Saline

\subsubsection{SN79 treatment blocks METH-induced astrogliosis}

Treatment with SN79 mitigated METH-induced increases in gfap expression at 12 and $24 \mathrm{~h}$ posttreatment. One-way ANOVA revealed significant differences between treatment groups at $12 \mathrm{~h}(\mathrm{~F}[3,38]$ $=16.20, \mathrm{p}<0.0001)$. Tukey's post hoc multiple comparison analysis revealed a significant difference 
between Saline/Saline and Saline/METH treated animals ( $q=8.50, p<0.001)$, similar to the results obtained during the time course experiment described above. SN79 pretreatment attenuated METHinduced increases of gfap expression at $12 \mathrm{~h}$ post-treatment $(q=7.28, p<0.001$ ) (Figure 8.2.A.). Furthermore, one-way ANOVA revealed significant differences between treatment groups at $24 \mathrm{~h}$ posttreatment $(F[3,39]=18.25, \mathrm{p}<0.0001)$. Post hoc Tukey's multiple comparison tests revealed that METH treatment resulted in a significant increase in striatal gfap mRNA expression compared to control $(q=$ 9.19, $p<0.001)$, an effect that was mitigated by treatment with SN79 $(q=6.99, p<0.001)$ (Figure 8.2.B.).

Similar results were obtained through protein level analysis utilizing immunohistochemistry (Figure 8.3.A.). METH treatment $(5 \mathrm{mg} / \mathrm{kg} \times 4)$ resulted in astrogliosis $72 \mathrm{~h}$ post-treatment. As shown in Figure 8.3.B., one-way ANOVA revealed differences between treatment groups in the number of reactive astrocytes within the striatum $(F[3,19]=27.75, p<0.0001)$. Tukey's multiple comparison tests revealed that METH significantly increased reactive astrocytes as compared to control $(q=10.99, p<$ 0.001). This effect was mitigated by treatment with SN79, indicative of a blockade of METH-induced reactive astrogliosis $(q=8.00, p<0.001)$.

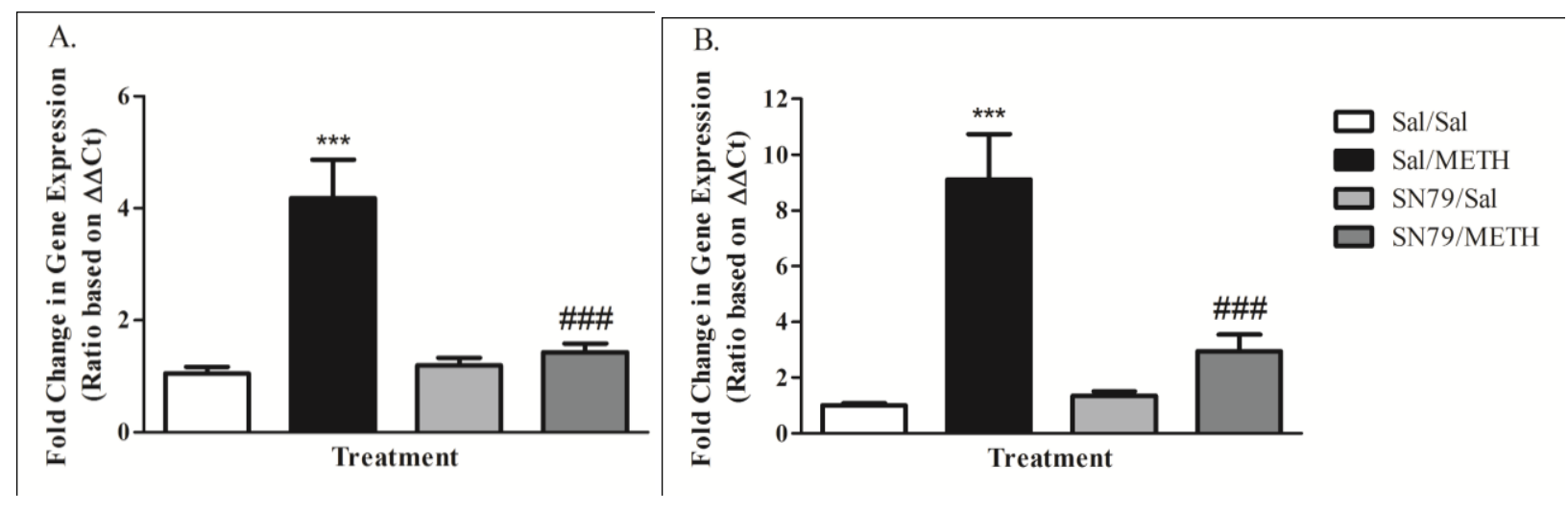

Figure 8.2. Treatment with SN79 ( $3 \mathrm{mg} / \mathrm{kg} \times 4)$ was found to attenuate METH-induced $(5 \mathrm{mg} / \mathrm{kg} \times 4)$ increases in striatal gfap mRNA expression at both 12 (A) and $24 \mathrm{~h}$ (B), indicating a blockade of astrocyte activation. One-way ANOVA, followed by post hoc Tukey's multiple comparison tests. ${ }^{* * *} p<0.001$, Sal/METH vs. Sal/Sal; \#\#\#p < 0.001, SN79/METH vs. Sal/METH. Sal = Saline 


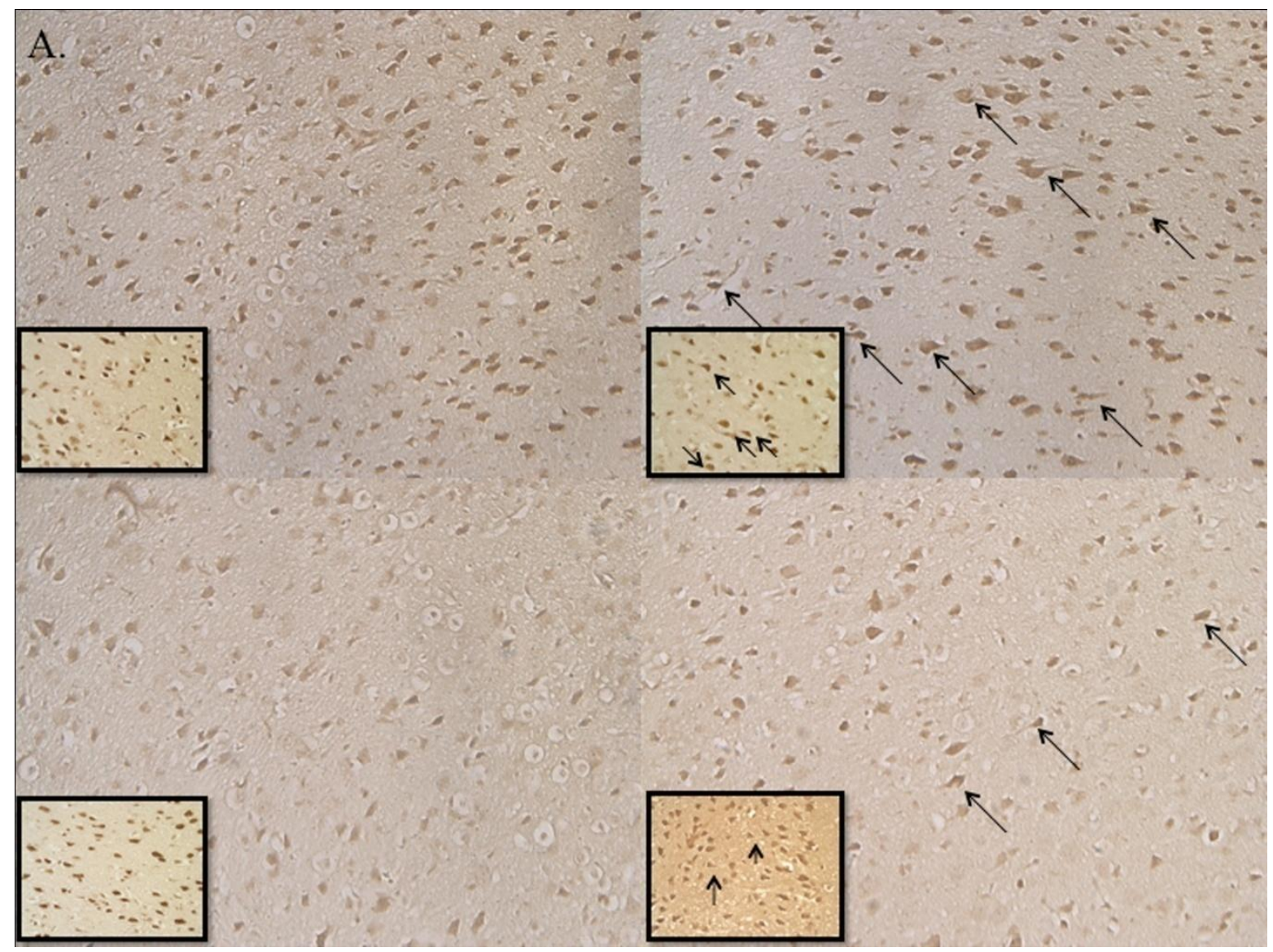

Figure 8.3.A. Qualitative images of astrocyte activation in response to METH $(5 \mathrm{mg} / \mathrm{kg} \times 4)$ and blockade by SN79 (3 mg/kg x 4) treatment. Clockwise from top left: Sal/Sal, Sal/METH, SN79/METH, SN79/Sal. Sal $=$ Saline. Arrows denote activated astrocytes that have undergone morphologic alterations.

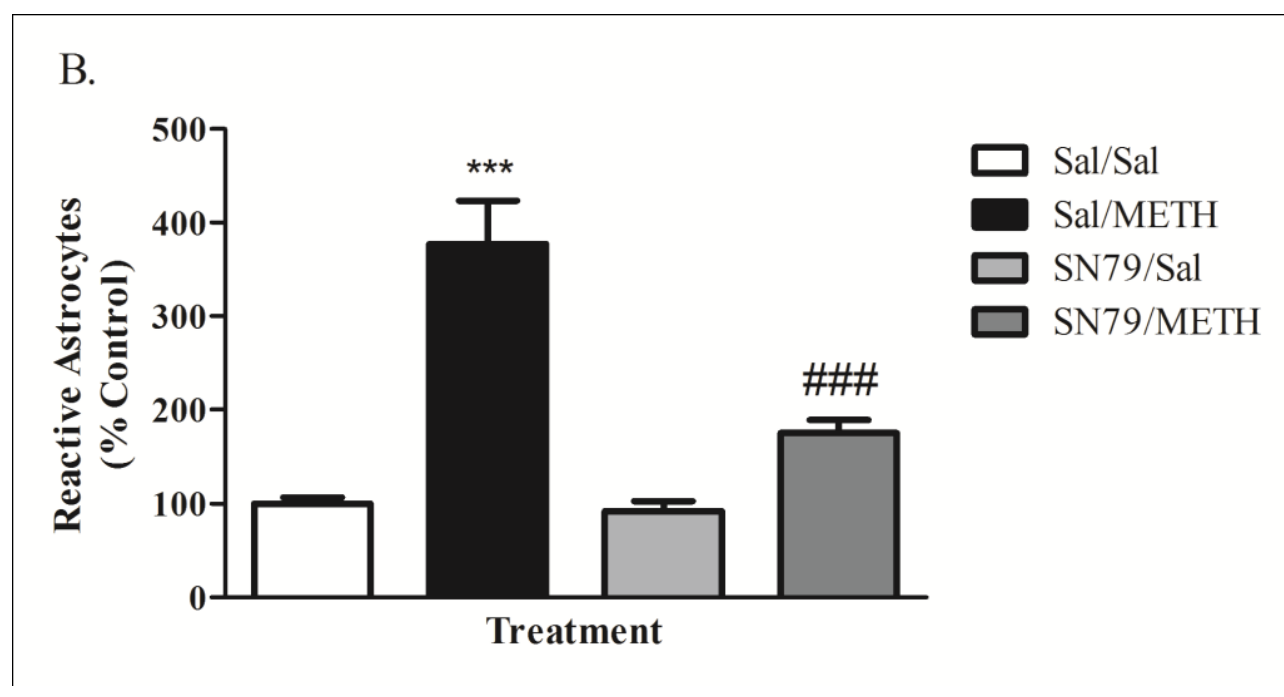

Figure 8.3.B. Quantification of the attenuation of methamphetamine (METH)-induced ( $5 \mathrm{mg} / \mathrm{kg} \times 4$ ) astrogliosis by SN79 treatment $(3 \mathrm{mg} / \mathrm{kg} \times 4)$. Sal = Saline. One-way ANOVA, followed by post hoc 
Tukey's multiple comparison tests. ${ }^{* * *} p<0.001$, Sal/METH vs. Sal/Sal; \#\#\#p <0.001, SN79/METH vs. Sal/METH.

\subsubsection{SN79 treatment mitigates METH-induced increases in OSMR expression}

METH treatment increased striatal mRNA osmr expression at 12 and $24 \mathrm{~h}$. One-way ANOVA revealed significant differences in osmr mRNA expression between treatment groups at $12 \mathrm{~h}$ posttreatment $(F[3,38]=36.06, p<0.0001)$. Post hoc Tukey's analysis showed that METH significantly increased striatal osmr expression as compared to saline-treated animals ( $q=12.94, p<0.001)$. This effect was mitigated by SN79 treatment $(q=9.74, p<0.001)$ (Figure 8.4.A.). Similar effects were seen at $24 \mathrm{~h}$ post-treatment. One-way ANOVA revealed differences in osmr expression between treatment groups $(F[3,39]=27.49, p<0.0001)$. Tukey's multiple comparison tests revealed that METH treatment increased osmr expression as compared to saline $(q=11.26, p<0.001)$, an effect that was mitigated by SN79 treatment $(q=8.92, p<0.001)$ (Figure 8.4.B.).
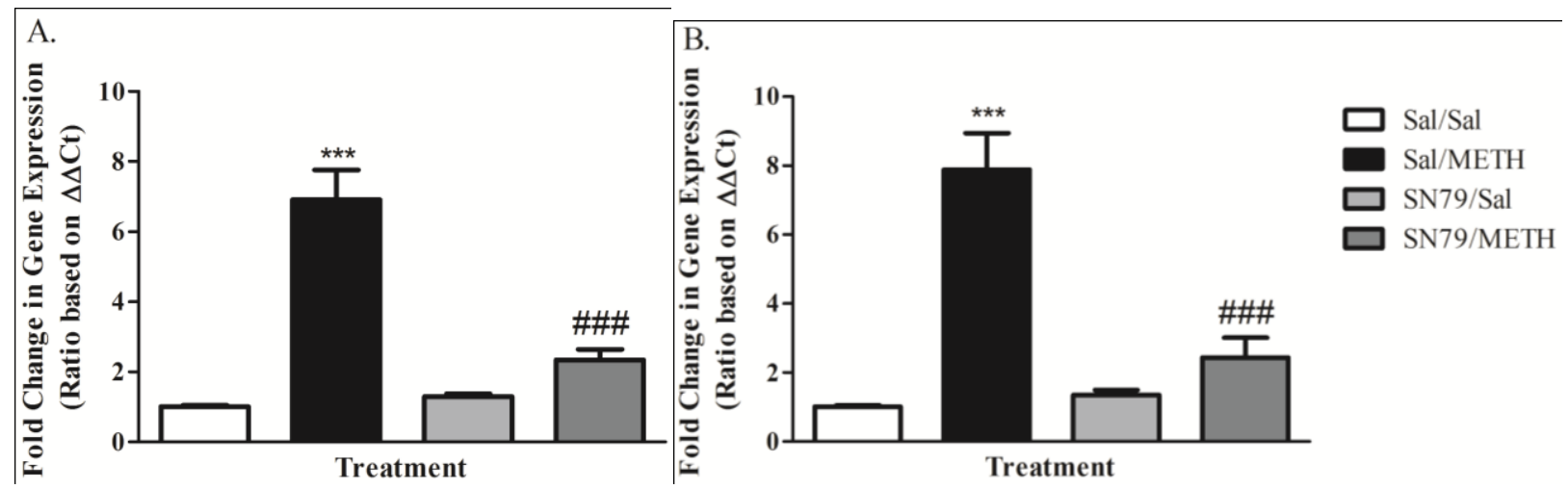

Figure 8.4. SN79 ( $3 \mathrm{mg} / \mathrm{kg} \times 4$ ) treatment blocks methamphetamine $(\mathrm{METH})$-induced $(5 \mathrm{mg} / \mathrm{kg} \times 4)$ increases in striatal osmr mRNA expression at 12 (A) and $24 \mathrm{~h}$ (B). One-way ANOVA, followed by post hoc Tukey's multiple comparison tests. Sal = Saline. ${ }^{* * *} p<0.001$, Sal/METH vs. Sal/Sal; \#\#\#p 0.001 , SN79/METH vs. Sal/METH.

To determine if increases in OSMR expression are occurring specifically in astrocytes located within the striatum, immunoflourescence studies were conducted to determine both levels of GFAP and 
OSMR protein between treatment groups, and the specific colocalization of increases in OSMR expression to astrocytes. One way ANOVA revealed differences between groups in GFAP protein expression at $72 \mathrm{~h}$ post-treatment $(F[3,19]=10.07, p<0.001)$. Tukey's post hoc analysis revealed that as expected METH treatment significantly increased striatal GFAP protein levels as compared to salinetreated controls $(q=6.67, p<0.01)$. SN79 treatment mitigated METH-induced increases in GFAP protein $(q=5.69, p<0.01)$ (Figure 8.5.A.). Furthermore, one-way ANOVA revealed significant differences in OSMR protein expression at $72 \mathrm{~h}$ post-treatment $(F[3,19]=11.74, p<0.001)$. Post hoc analysis using Tukey's multiple comparison tests showed that METH treatment increased OSMR expression compared to saline-treated controls $(q=7.63, p<0.001)$. SN79 treatment blocked METH-induced increases in OSMR expression $(q=5.89, p<0.01)$ (Figure 8.5.B.).
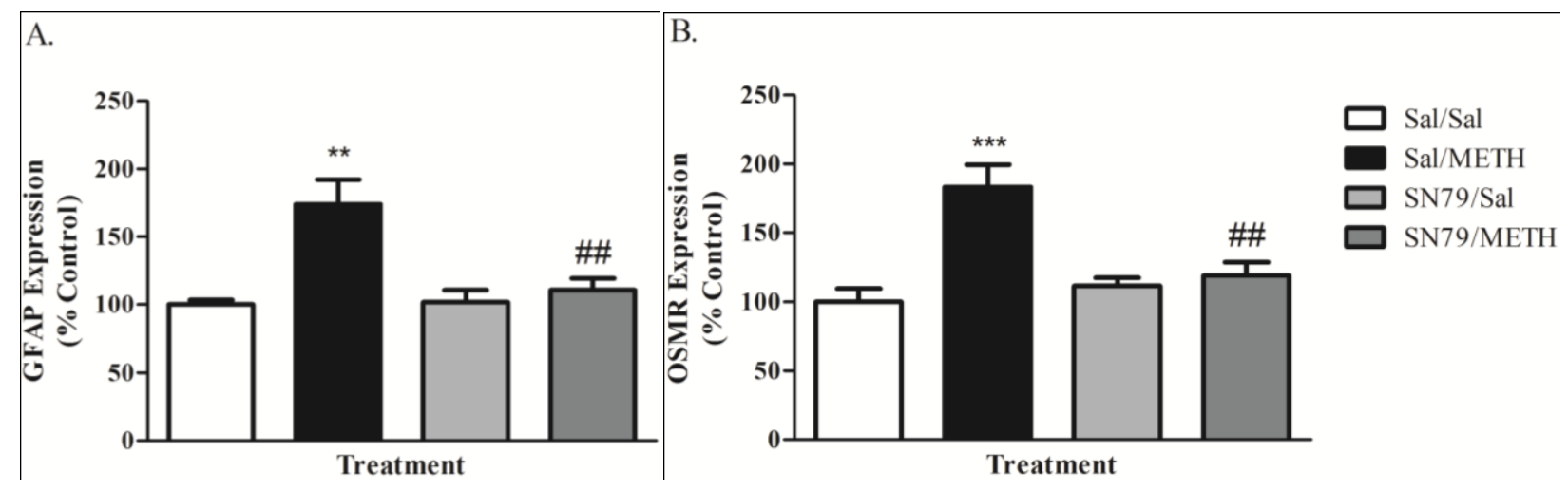


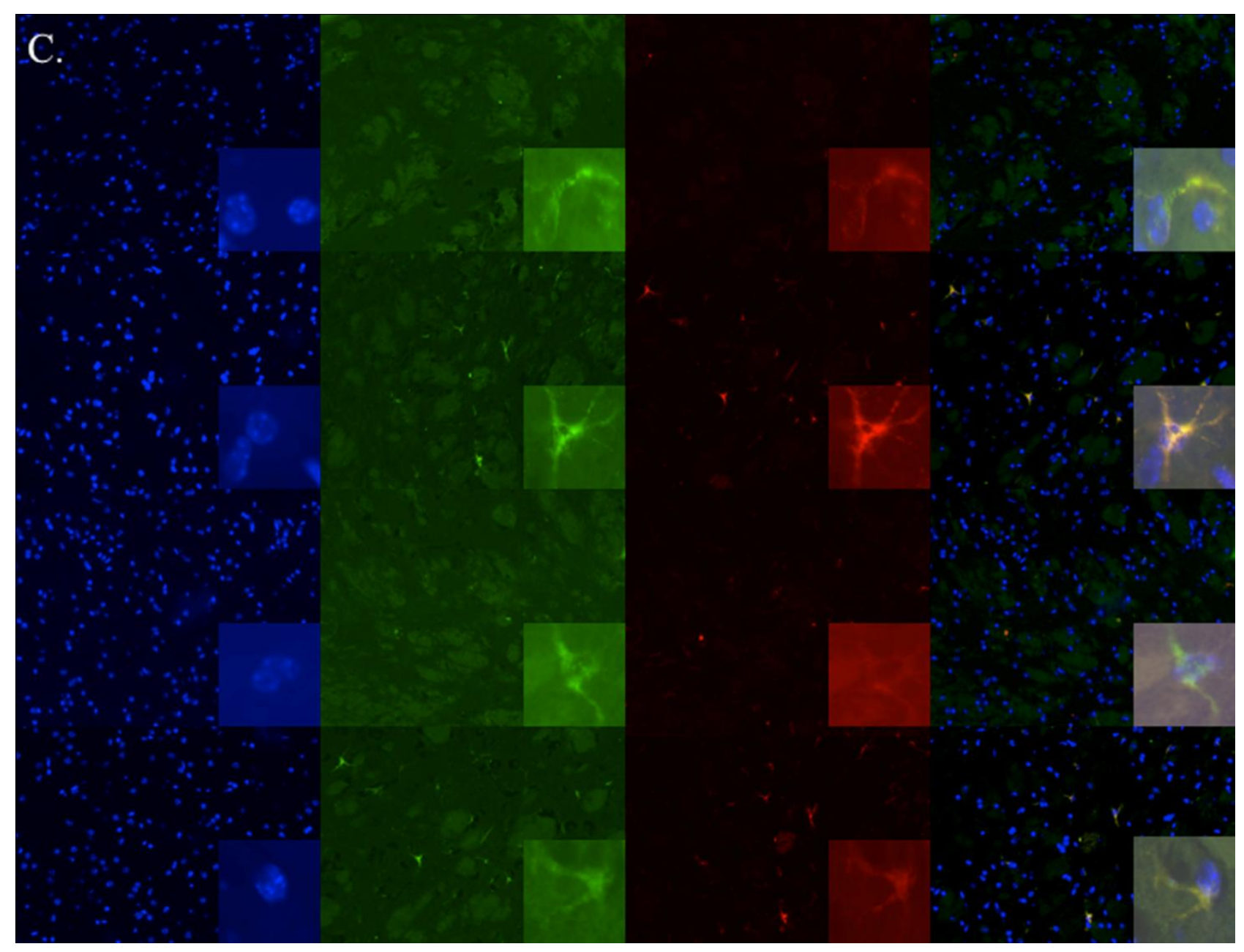

Figure 8.5. (A) Methamphetamine (METH) $(5 \mathrm{mg} / \mathrm{kg} \times 4)$ treatment increases the expression of striatal GFAP, indicating significant astrogliosis. SN79 $(3 \mathrm{mg} / \mathrm{kg} \times 4)$ treatment blocked METH-induced increases in GFAP expression. (B) METH treatment increased OSMR expression in striatal astrocytes, an effect mitigated by SN79 treatment. One-way ANOVA, followed by post hoc Tukey's multiple comparison tests. ${ }^{* *} \mathrm{p}<0.01, * * * \mathrm{p}<0.001$, Sal/METH vs. Sal/Sal; \#\#p < 0.01, SN79/METH vs. Sal/METH (C) Representative images showing increases in GFAP expression and increases in astrocytic OSMR expression in the striatum by treatment group. Top to bottom: Sal/Sal, Sal/METH, SN79/Sal, SN79/METH Left to right: DAPI nuclear staining, GFAP, OSMR, overlay image. Sal = Saline.

Importantly, as shown in Figure 8.5.C., increases in GFAP and OSMR induced by METH were colocalized specifically to astrocytes within the striatum. METH treatment resulted in an increase in OSMR specifically in GFAP-labeled astrocytes, an effect that was blocked by SN79 treatment.

\subsubsection{SN79 treatment blocks METH-induced phosphorylation of STAT3}


Western blotting of bilateral striatal tissue $12 \mathrm{~h}$ post-treatment was conducted to determine the effect of METH on the phosphorylation of STAT3 (Tyr-705) and total STAT3 levels that has previously been linked to astrocyte activation by neurotoxicants, including METH in vitro (Hebert and O'Callaghan, 2000; Sriram et al., 2004). One-way ANOVA revealed significant differences in pSTAT3 levels between groups $(F[3,31]=5.69, p<0.01)$. Tukey's post hoc analysis revealed that METH treatment significantly increased the phosphorylation of STAT3 as compared to saline-treated controls at $12 \mathrm{~h}(\mathrm{q}=4.90, \mathrm{p}<$ 0.01). SN79 treatment blocked the METH-induced phosphorylation of STAT3 ( $q=4.34, p<0.05$ ) (Figure 7.6.A.). Conversely, there were no significant differences found in total STAT3 levels in the striatum between any of the treatment groups (one-way ANOVA, n.s.) (Figure 8.6.B.).

A.
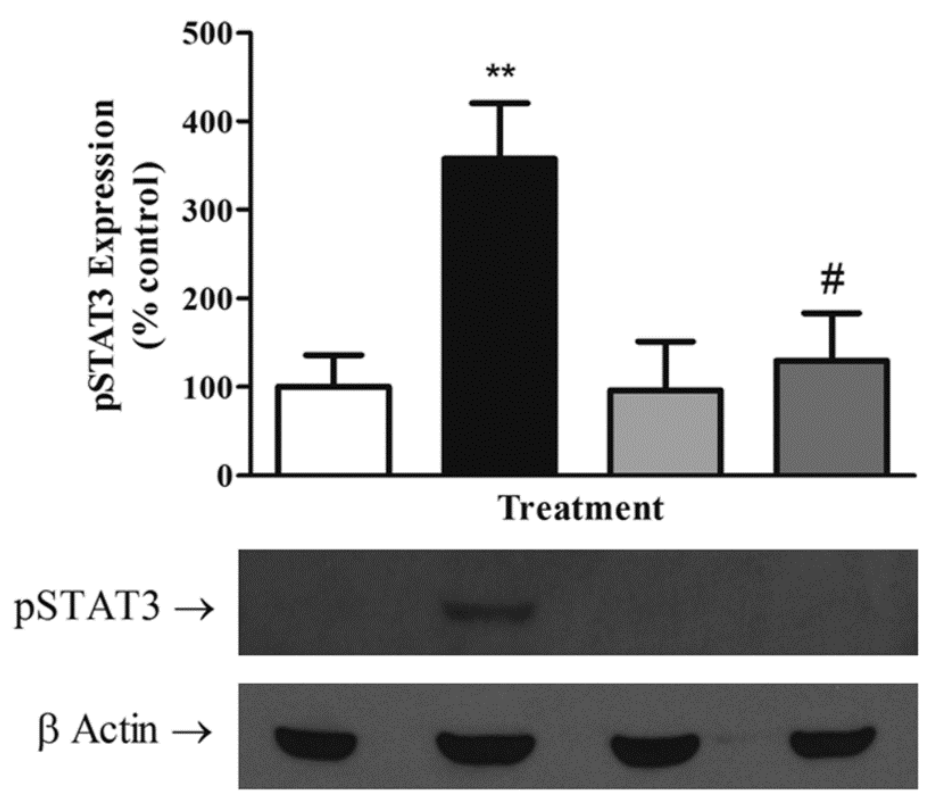
B.

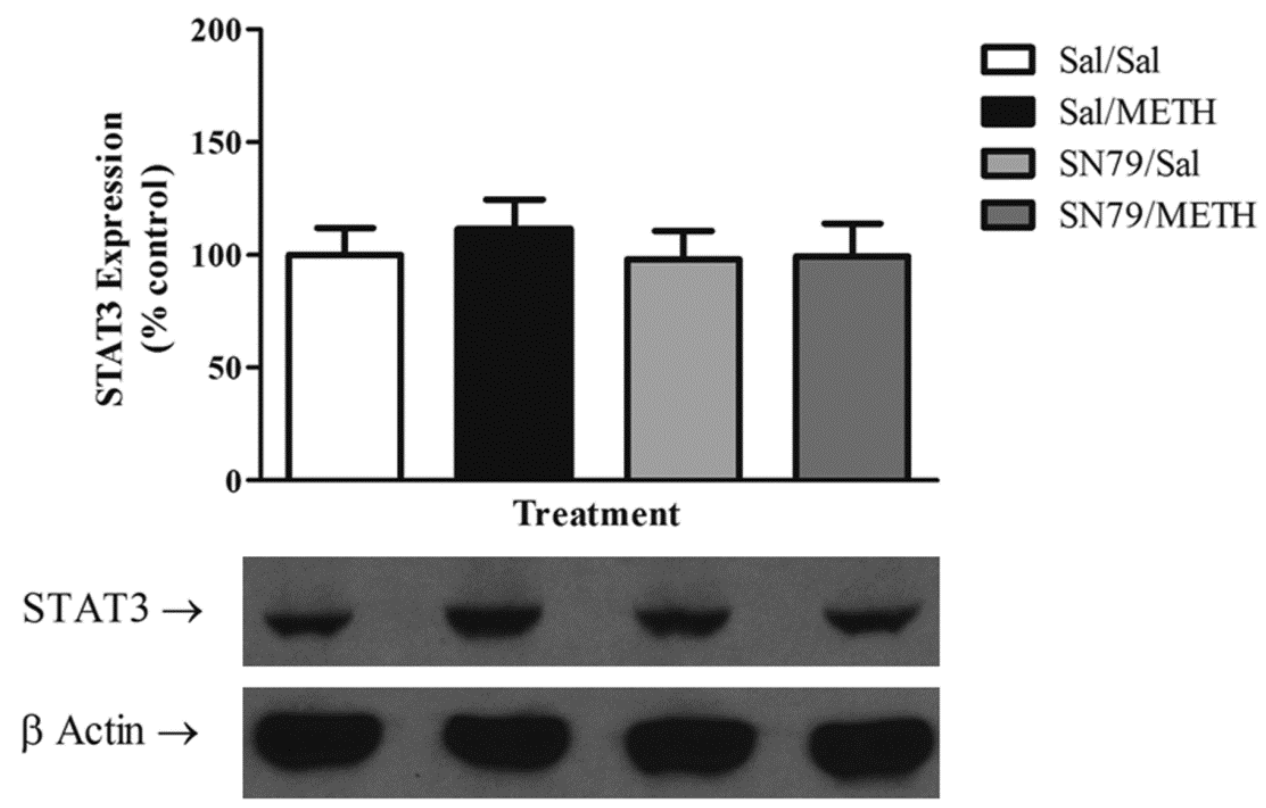

Figure 8.6. SN79 mitigates methamphetamine (METH)-induced increases in the phosphorylation of STAT3 (Tyr-705). (A) METH treatment $(5 \mathrm{mg} / \mathrm{kg} \times 4$ ) increases the phosphorylation of STAT3 (Tyr-705) at $12 \mathrm{~h}$; an effect that is mitigated by treatment with SN79 ( $3 \mathrm{mg} / \mathrm{kg} \times 4)$. One-way ANOVA followed by post hoc Tukey's multiple comparison tests. Sal = Saline. ${ }^{* *} p<0.01$, Sal/METH vs. Sal/Sal; \#p $<0.05$, SN79/METH vs. Sal/METH. (B) No differences were detected between any of the treatment groups in total STAT3 expression.

\subsubsection{SN79 treatment attenuates METH-induced neuronal degeneration}

One-way ANOVA revealed significant differences in the number of degenerating neurons within the striatum between treatment groups $72 \mathrm{~h}$ post-treatment $(F[3,19]=11.96, p<0.001)$, as determined by Flouro-Jade B staining. METH treatment significantly increased the number of degenerating neurons in the striatum as compared to saline-treated controls (Tukey's post hoc analysis, $q=8.12, p<0.001)$. SN79 treatment mitigated METH-induced increases in neuronal degeneration $(q=$ 5.20, $\mathrm{p}<0.01$ ) (Figure 8.7.A. and 8.7.B.). 

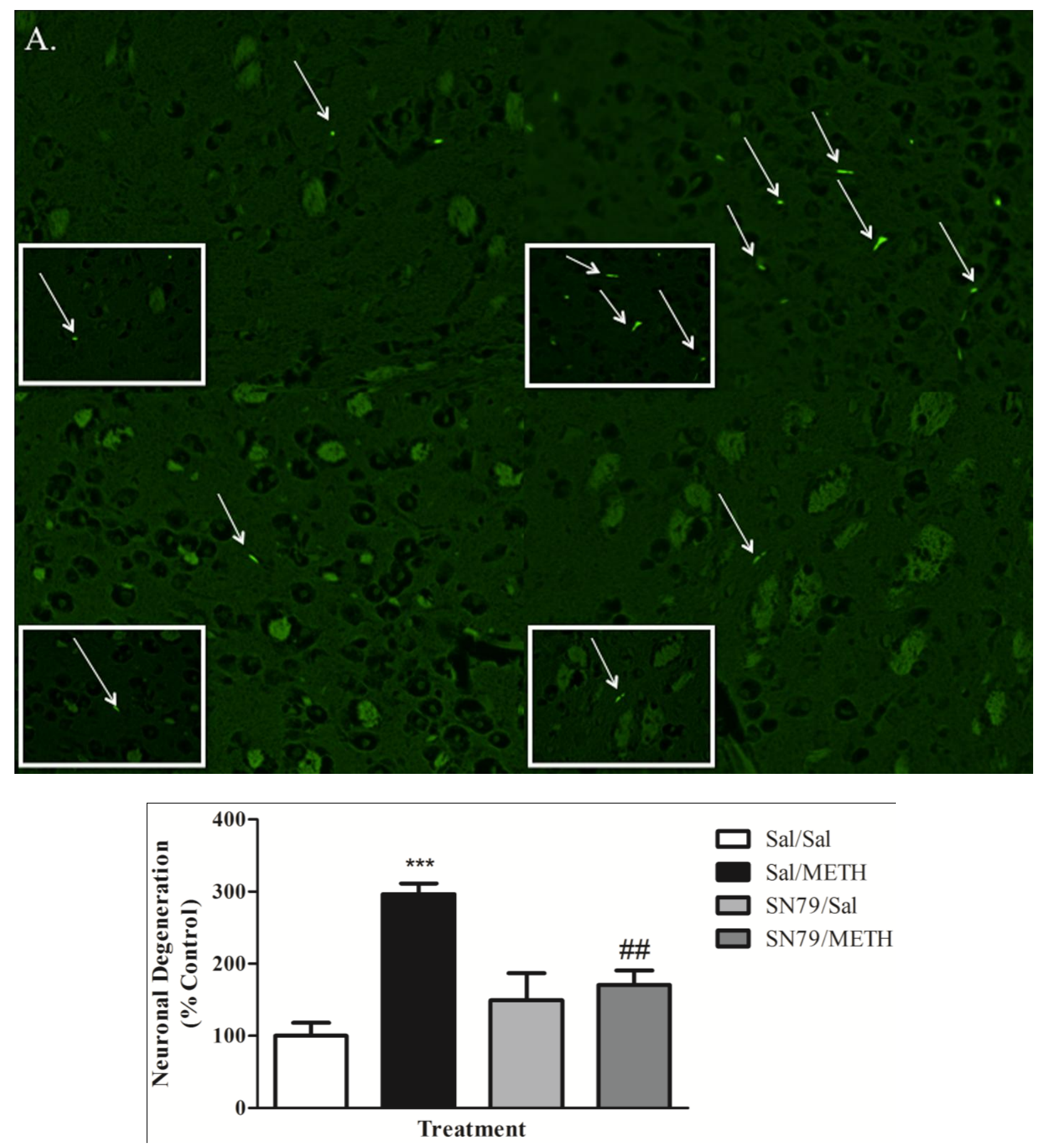

Figure 8.7. SN79 ( $3 \mathrm{mg} / \mathrm{kg} \times 4$ ) treatment attenuates methamphetamine (METH)-induced (5 mg/kg x 4) neuronal degeneration in the striatum as measured by Fluoro-Jade B staining. (A) Representative images of Fluoro-Jade $\mathrm{B}$ labeled degenerating neurons in the striatum for each respective treatment group. Clockwise from top left: Sal/Sal, Sal/METH, SN79/METH, SN79/Sal. Sal = Saline. (B) Quantification of striatal neuronal degeneration by treatment group. One-way ANOVA, followed by post hoc Tukey's multiple comparison tests. ${ }^{* * *} p<0.001$, Sal/METH vs. Sal/Sal; \#\#p <0.01, SN79/METH vs. Sal/METH 


\subsection{Discussion}

The current study provides evidence that the sigma receptor antagonist and potential drug development candidate, SN79, mitigates METH-induced astrogliosis and neuronal degeneration in the striatum. Additionally, the current study provides evidence that the ability of SN79 to attenuate METHinduced astrogliosis is mediated through a blockade of STAT3 phosphorylation initiated by OSMR signaling.

Our findings are consistent with earlier reports that METH-induced activation of astrocytes involves OSM interactions with OSMR and subsequent induction of JAK2/STAT3 signaling (Chen and Benveniste 2004; Hebert and O'Callaghan 2000; Heinrich et al. 2003). This induction of STAT3 phosphorylation through IL-6-type signaling can result in a variety of genetic changes, including increases in GFAP expression that are associated with astrocyte activation (Hebert and O'Callaghan 2000; Oliva et al. 2012; Sriram et al. 2004; Zhong et al. 1994). The current study is the first to show that treatment with a sigma receptor antagonist mitigates METH-induced increases in signaling through this pathway and thereby counteracts METH-induced astrogliosis.

The precise mechanism(s) by which SN79 modulates this pathway has yet to be fully elucidated. It is possible that SN79 is blocking METH-induced increases in OSM, thereby leading to a reduction of signaling through OSMR. A number of labs have shown that METH treatment results in an increase in striatal expression levels of osm mRNA (Cadet et al. 2002; Kelly et al. 2012; Kuhn et al. 2006). Furthermore, we have also shown that SN79 mitigates METH-induced increases in striatal osm mRNA expression (Robson et al. 2013). This could lead to a blockade of METH-induced STAT3 phosphorylation by decreasing signaling specifically through OSMR in astrocytes and leading ultimately to a reduction in METH-induced GFAP upregulation and astrogliosis as seen in the current study. 


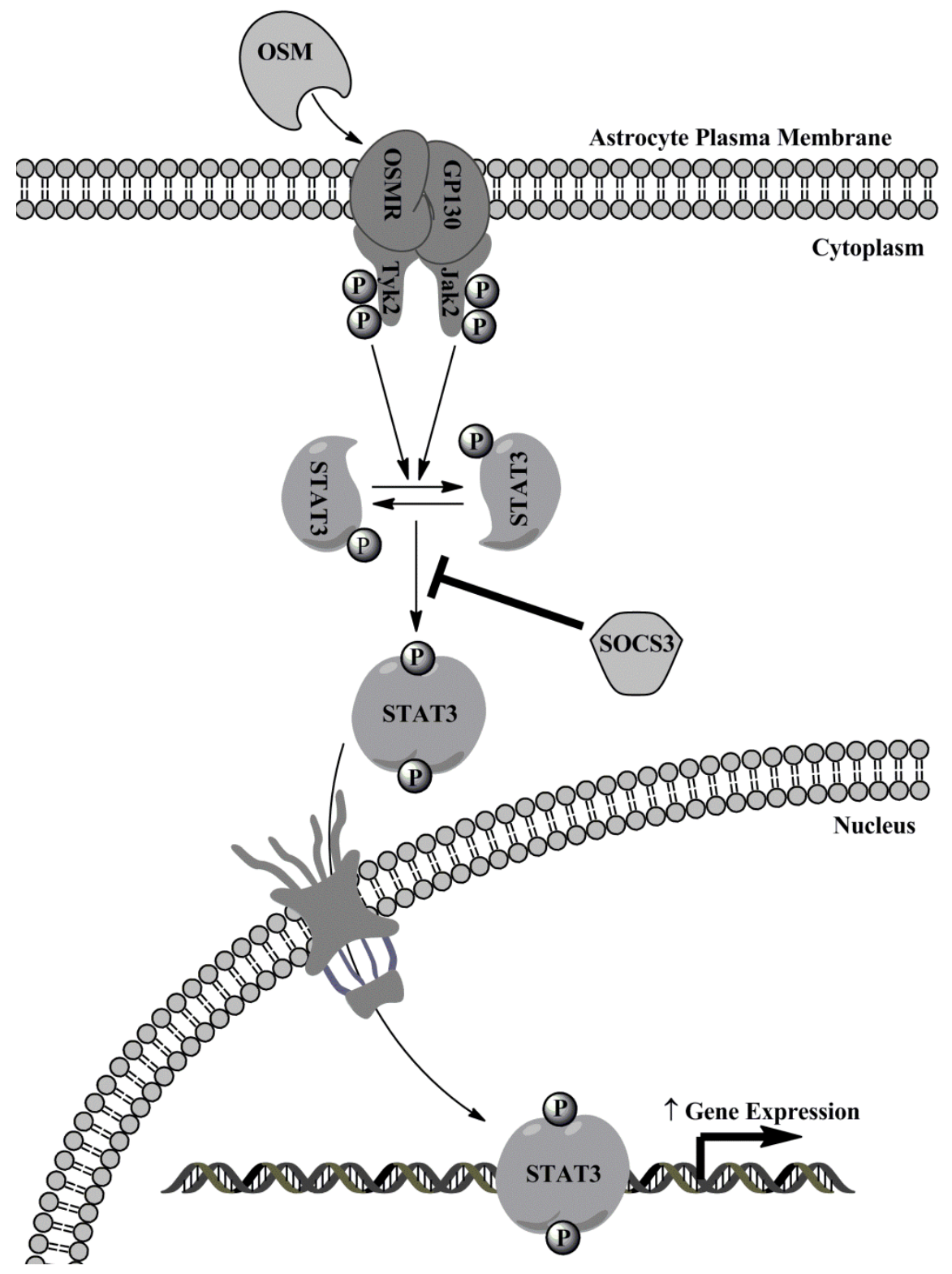

Figure 8.8. OSM signaling is known to occur through OSMR/gp130 heterodimers resulting in JAK2/STAT3 signaling. The resulting phosphorylation of STAT3 (Tyr-705) causes STAT3 to homodimerize and subsequently induces gene expression alterations. This process is believed to be critical to astrogliosis in response to neurotoxicants such as METH.

Another potential explanation is that sigma receptor modulation within astrocytes is having an effect downstream of OSMR on STAT3 phosphorylation directly. Sigma receptors themselves and sigma receptor ligands have previously been shown to modulate the function of astrocytes in vitro (Ruscher et 
al. 2011). Furthermore, sigma receptor ligands have been shown to modulate protein expression within cultured astrocytes (Prezzavento et al. 2007), in addition to dose-dependently blocking reductions in astrocytic ATP resulting from hypoxia in vitro (Klouz et al. 2003). It is thus possible that sigma receptors within astrocytes are altering astrocytic responses to CNS insults that result in astrogliosis.

One particular protein involved in the negative feedback of STAT3 signaling in astrocytes is suppressor of cytokine signaling 3 (SOCS3) (Baker et al. 2008). Astrocytic OSM signaling results in an upregulation of SOCS3 thereby suppressing the inflammatory actions of OSM, including the phosphorylation of STAT3 (Baker et al. 2008). Three distinct pathways are believed to contribute to these effects, including the phosphorylation of STAT3, ERK1/2 and JNK pathways (Baker et al. 2008). Although SN79 treatment was found to mitigate METH-induced increases in STAT3 phosphorylation, it may be possible that modulation of ERK1/2 and JNK pathways are contributing to increases in SOCS3, thereby leading to the blockade of STAT3 phosphorylation seen in the current report. Supporting this hypothesis are previous studies showing the modulation of ERK1/2 and JNK signaling by various sigma receptor ligands (Cantarella et al. 2007; Nishimura et al. 2008; Son and Kwon 2010; Tan et al. 2010; Tuerxun et al. 2010), although their effects specifically within astrocytes on these signaling pathways are currently unknown.

Our study provides evidence that METH-induced STAT3 phosphorylation occurs prior to the induction of increases in GFAP and OSMR expression elicited by METH. Previous studies have shown that maximal increases in GFAP post-treatment with METH occur between 48 and $72 \mathrm{~h}$, indicative of a maximal increase in astrocyte activation (Hebert and O'Callaghan 2000; Kelly et al. 2012; Sriram et al. 2006). Moreover, it is known that maximal METH-induced increases in the phosphorylation of STAT3 occur prior to these effects (between 12 and 24 h) (Hebert and O'Callaghan 2000). Although protein changes in OSMR expression are protracted as compared to STAT3 phosphorylation, osm mRNA expression increases post-METH treatment are rapid (Kelly et al. 2012), suggesting that signaling 
through this receptor may result in a positive feedback loop, whereby signaling increases the expression of the receptor. In the current study, increases in osmr mRNA and protein elicited by METH were detected at time points corresponding to and also after the phosphorylation of STAT3, respectively. Interestingly, increases in OSMR expression also coincided with increases in GFAP mRNA and protein. It is possible that initial OSM signaling through OSMR results in a positive feedback loop, where the increases OSMR expression result in increased signaling through astrocytic OSMR thereby potentiating its effects. It is known that OSM signaling can alter gene expression in astrocytes in a biphasic manner; however the specificity of STAT3 signaling in mediating these genetic alterations has yet to be confirmed (Van Wagoner et al. 2000). Furthermore, the second portion of this biphasic response has been reported to require de novo protein synthesis (Van Wagoner et al. 2000), indicating that the genetic alterations elicited through this pathway (such as increases in gfap mRNA expression) may require increased expression of specific proteins, including OSMR. In support of this hypothesis, OSM signaling has previously been shown to increase levels of OSMR $\beta$ receptor subunits by enhancing the level of synthesis of these receptor subunits (Blanchard et al. 2001). This biphasic response could potentially be a manifestation of a positive feedback loop resulting in further increases in OSMR/gp130 signaling and increases in GFAP expression/astrocyte activation (Nakashima et al. 1999). The mitigation of METHinduced increases in astrocytic OSMR by SN79 treatment could result in a blockade of the second half of this biphasic response, thereby shunting increases in GFAP expression elicited through OSMR/gp130 signaling.

In addition to a potential role in neurotoxicity, astrocytes themselves are hypothesized to be involved in synaptic plasticity and may play large roles in the control of synaptic activity (Nedergaard and Verkhratsky 2012; Ullian et al. 2004). Substantial evidence points to astrocytes being involved in many aspects of synapse formation, maintenance and stability and they may be involved in activity dependent synapse formation (Ullian et al. 2004). These effects are hypothesized to be involved in the 
actions of several drugs of abuse including METH and the modulation of astrocytes is believed to represent a novel target for the production of pharmacotherapies aimed at treating drug abuse (Cooper et al. 2012; Miguel-Hidalgo 2009; Narita et al. 2008; Rolan et al. 2009). Evidence of astrocyte modulation being involved in the behavioral actions of METH supports a role of these cells in the effects of the drug beyond neurotoxicity. Previous reports have shown that METH-induced astrocyte activation is associated with METH-induced behavioral sensitization, a behavioral assay believed to be associated with neuroplastic changes elicited by drugs of abuse (Chen et al. 2009; Narita et al. 2006; Narita et al. 2005; Narita et al. 2008). Interestingly, sigma receptor ligands, have previously been shown to block the development and expression of behavioral sensitization to psychostimulants, including METH (Seminerio et al. 2012b; Ujike et al. 1992; Ujike et al. 1996; Xu et al. 2010); however, whether this effect is associated with astrocyte activation has yet to be determined.

It should be noted that currently, there are no available pharmacologic agents targeting OSMR specifically and these results await confirmation through the use of genetic manipulations to conclusively confirm that OSMR signaling is specifically involved in METH-induced astrocyte activation. Furthermore, the current study lacks genetic manipulations of sigma receptors to conclusively determine that the effects seen are due exclusively to sigma receptor modulation. SN79, the particular sigma ligand used during the current study however, has been utilized previously to study the role of sigma receptors in the actions of psychostimulants such as METH (Kaushal et al. 2011; Kaushal et al. 2012). Additionally, this ligand represents a potential drug development candidate aimed at treating the negative side effects of METH abuse such as neurotoxicity, as it has not only desirable pharmacologic effects, but also desirable pharmacokinetic parameters as well (Kaushal et al. 2011). These effects of SN79, combined with its ability to mitigate METH-induced astrocyte activation, neuronal degeneration and STAT3 phosphorylation as described within the current study make it a viable candidate for future drug development. Future studies aimed at the further delineation of the molecular mechanisms by 
which SN79 and other sigma receptor ligands mitigate the neurotoxic consequences of METH are certainly warranted.

\subsection{Contributions}

Matthew Robson conducted all in vivo studies, quantitative real-time PCR and western blots.

Ryan Turner and Zachary Naser performed immunohistochemistry and immunofluorescence studies and data obtained was analyzed by Matthew Robson. James O'Callaghan and NIOSH graciously allowed the use of a small rodent microwave irradiation unit. 


\section{Chapter 9}

Summary and Conclusions 


\subsection{Summary:}

\subsubsection{Sigma receptor involvement in the antidepressant-like effects of ketamine}

The studies contained within the current dissertation have thus demonstrated:

- Sigma receptor modulation is involved in specific aspects of the antidepressant-like effects of ketamine, a drug known to have rapid and persistent antidepressant effects.

- Sigma receptors are involved in the ability of ketamine to potentiate the effects of NGF on neurite outgrowth in vitro, an effect indicative of antidepressant-like actions.

\subsubsection{The involvement of sigma receptors in neurotoxic actions of METH}

- The ability of sigma receptor antagonists to mitigate METH-induced dopaminergic neurotoxicity is correlated to their ability to block METH-induced increases in core body temperature.

- AC927, a protective, selective sigma receptor antagonist, blocks specific gene expression changes in the striatum elicited by METH

- SN79, a protective sigma receptor antagonist, is unable to alter METH-induced PERK-mediated ER stress in an in vitro model of neurotoxicity.

- SN79 mitigates METH-induced microglial activation in the striatum, an effect believed to contribute to the ability of METH to elicit neurotoxicity.

- SN79 blocks METH-induced striatal increases in IL-6-type cytokine expression, an effect indicative of a blockade of neuroinflammation.

- Furthermore, SN79 treatment blocks METH-induced astrocyte activation and OSMR/gp130 signaling. 


\subsection{Conclusions:}

The studies included in this dissertation have accomplished two primary aims: 1) determined that ketamine exerts effects on neurite outgrowth through the modulation of sigma receptors and 2) begun to elucidate potential mechanisms by which sigma receptor antagonists mitigate the neurotoxic actions of METH.

Our studies effectively show that ketamine interacts with both subtypes of sigma receptors and although it does not appear the antidepressant-like effects of ketamine in the forced swim test involve sigma receptors, the ability of ketamine to potentiate NGF-induced neurite outgrowth in vitro is dependent upon sigma receptor modulation. Currently, it is unclear how ketamine exerts its antidepressant effects and these studies provide a novel target by which ketamine modulates neuroplastic changes believed to be relevant to the actions of antidepressants. Future characterization of the molecular signaling pathways involved in these effects could point to new molecular targets for the production of pharmacotherapies with antidepressant actions similar to ketamine while also having reduced side effect liabilities.

Furthermore, these studies have begun to determine how sigma receptor antagonists mitigate the neurotoxic actions of METH. These studies are important as sigma receptors are believed to be a novel target for the production of pharmaceuticals aimed at countering the negative side effects and addiction liability to psychostimulants including METH. Our studies determined that sigma receptor antagonists are modulating glial cell function and neuroinflammation as a result of neurotoxic METH exposure. Glial cell activation and cytokine release, indicating neuroinflammation, is implicated in the ability of METH to cause neurotoxicity. Furthermore, an increase in neuroinflammation is present in several neurodegenerative disorders and may represent a future target for novel therapeutics aimed at treating these diseases. Our studies provide evidence that sigma receptor ligands may represent a class of novel therapeutics that are able to reduce glial cell activation and neuroinflammation as a result of 
various CNS disorders. Future studies determining whether these effects are causal in the ability of sigma receptor antagonists to mitigate the neurotoxic actions of METH are warranted. Additionally, the determination as to whether the ability of sigma receptor antagonists to mitigate glial cell function and neuroinflammation are specific to METH, or whether this is a generalized effect across various models of neurodegenerative disorders, are certainly warranted. 


\section{REFERENCES}

aan het Rot, M., Collins, K.A., Murrough, J.W., Perez, A.M., Reich, D.L., Charney, D.S., Mathew, S.J., 2010. Safety and efficacy of repeated-dose intravenous ketamine for treatment-resistant depression. Biol Psychiatry 67, 139-145.

Adler, H.T., Chinery, R., Wu, D.Y., Kussick, S.J., Payne, J.M., Fornace, A.J., Jr., Tkachuk, D.C., 1999.

Leukemic HRX fusion proteins inhibit GADD34-induced apoptosis and associate with the GADD34 and hSNF5/INI1 proteins. Mol Cell Biol 19, 7050-7060.

Ajmo, C.T., Jr., Vernon, D.O., Collier, L., Pennypacker, K.R., Cuevas, J., 2006. Sigma receptor activation reduces infarct size at 24 hours after permanent middle cerebral artery occlusion in rats. Curr Neurovasc Res 3, 89-98.

Albers, D.S., Sonsalla, P.K., 1995. Methamphetamine-induced hyperthermia and dopaminergic neurotoxicity in mice: pharmacological profile of protective and nonprotective agents. J Pharmacol Exp Ther 275, 1104-1114.

Alonso, G., Phan, V., Guillemain, I., Saunier, M., Legrand, A., Anoal, M., Maurice, T., 2000.

Immunocytochemical localization of the sigma(1) receptor in the adult rat central nervous system. Neuroscience 97, 155-170.

Autry, A.E., Adachi, M., Nosyreva, E., Na, E.S., Los, M.F., Cheng, P.F., Kavalali, E.T., Monteggia, L.M., 2011. NMDA receptor blockade at rest triggers rapid behavioural antidepressant responses. Nature 475, 91-95.

Aydar, E., Palmer, C.P., Klyachko, V.A., Jackson, M.B., 2002. The sigma receptor as a ligand-regulated auxiliary potassium channel subunit. Neuron 34, 399-410.

Baker, B.J., Qin, H., Benveniste, E.N., 2008. Molecular basis of oncostatin M-induced SOCS-3 expression in astrocytes. Glia 56, 1250-1262.

Ball, J.R., Kiloh, L.G., 1959. A controlled trial of imipramine in treatment of depressive states. Br Med J 2, 1052-1055.

Basianetto, S., Roquier, L., Perrault, G., Sanger, D.J., 1995. DTG-induced circling behaviour in rats may involve the interaction between sigma sites and nigro-striatal dopaminergic pathways.

Neuropharmacology 34, 281-287.

Battaglia, G., Fornai, F., Busceti, C.L., Aloisi, G., Cerrito, F., De Blasi, A., Melchiorri, D., Nicoletti, F., 2002. Selective blockade of mGlu5 metabotropic glutamate receptors is protective against methamphetamine neurotoxicity. J Neurosci 22, 2135-2141.

Beauvais, G., Atwell, K., Jayanthi, S., Ladenheim, B., Cadet, J.L., 2011. Involvement of dopamine receptors in binge methamphetamine-induced activation of endoplasmic reticulum and mitochondrial stress pathways. PLoS One 6, e28946.

Bechtholt-Gompf, A.S., KL. John, CS. Kang, HH. Carlezon Jr, WA. Cohen, BM. Ongur, D., 2011. CD-1 and Balb/cJ mice do not show enduring antidepressant-like effects of ketamine in tests of acture antidepressant efficacy. Psychopharmacology Article in press.

Bergeron, R., de Montigny, C., Debonnel, G., 1995. Biphasic effects of sigma ligands on the neuronal response to N-methyl-D-aspartate. Naunyn Schmiedebergs Arch Pharmacol 351, 252-260.

Bergeron, R., Debonnel, G., De Montigny, C., 1993. Modification of the N-methyl-D-aspartate response by antidepressant sigma receptor ligands. Eur J Pharmacol 240, 319-323.

Bermack, J., Lavoie, N., Dryver, E., Debonnel, G., 2002. Effects of sigma ligands on NMDA receptor function in the bulbectomy model of depression: a behavioural study in the rat. Int J Neuropsychopharmacol 5, 53-62.

Bermack, J.E., Debonnel, G., 2001. Modulation of serotonergic neurotransmission by short- and longterm treatments with sigma ligands. Br J Pharmacol 134, 691-699. 
Bermack, J.E., Debonnel, G., 2005. Distinct modulatory roles of sigma receptor subtypes on glutamatergic responses in the dorsal hippocampus. Synapse 55, 37-44.

Berman, R.M., Cappiello, A., Anand, A., Oren, D.A., Heninger, G.R., Charney, D.S., Krystal, J.H., 2000. Antidepressant effects of ketamine in depressed patients. Biol Psychiatry 47, 351-354.

Berton, O., Nestler, E.J., 2006. New approaches to antidepressant drug discovery: beyond monoamines. Nat Rev Neurosci 7, 137-151.

Blanchard, F., Wang, Y., Kinzie, E., Duplomb, L., Godard, A., Baumann, H., 2001. Oncostatin M regulates the synthesis and turnover of gp130, leukemia inhibitory factor receptor alpha, and oncostatin $\mathrm{M}$ receptor beta by distinct mechanisms. J Biol Chem 276, 47038-47045.

Blier, P., Bouchard, C., 1994. Modulation of 5-HT release in the guinea-pig brain following long-term administration of antidepressant drugs. Br J Pharmacol 113, 485-495.

Booth, R.G., Baldessarini, R.J., 1991. (+)-6,7-benzomorphan sigma ligands stimulate dopamine synthesis in rat corpus striatum tissue. Brain Res 557, 349-352.

Bourrie, B., Benoit, J.M., Derocq, J.M., Esclangon, M., Thomas, C., Le Fur, G., Casellas, P., 1996. A sigma ligand, SR 31747A, potently modulates Staphylococcal enterotoxin B-induced cytokine production in mice. Immunology 88, 389-393.

Bowen, W.D., 2000. Sigma receptors: recent advances and new clinical potentials. Pharm Acta Helv 74, 211-218.

Bowyer, J.F., Davies, D.L., Schmued, L., Broening, H.W., Newport, G.D., Slikker, W., Jr., Holson, R.R., 1994. Further studies of the role of hyperthermia in methamphetamine neurotoxicity. J Pharmacol Exp Ther 268, 1571-1580.

Bowyer, J.F., Tank, A.W., Newport, G.D., Slikker, W., Jr., Ali, S.F., Holson, R.R., 1992. The influence of environmental temperature on the transient effects of methamphetamine on dopamine levels and dopamine release in rat striatum. J Pharmacol Exp Ther 260, 817-824.

Bradford, M.M., 1976. A rapid and sensitive method for the quantitation of microgram quantities of protein utilizing the principle of protein-dye binding. Anal Biochem 72, 248-254.

Brunswick, D.J., Benmansour, S., Tejani-Butt, S.M., Hauptmann, M., 1992. Effects of high-dose methamphetamine on monoamine uptake sites in rat brain measured by quantitative autoradiography. Synapse 11, 287-293.

Cadet, J.L., Jayanthi, S., Deng, X., 2003. Speed kills: cellular and molecular bases of methamphetamineinduced nerve terminal degeneration and neuronal apoptosis. FASEB J 17, 1775-1788.

Cadet, J.L., Krasnova, I.N., 2009. Molecular bases of methamphetamine-induced neurodegeneration. Int Rev Neurobiol 88, 101-119.

Cadet, J.L., Krasnova, I.N., Jayanthi, S., Lyles, J., 2007. Neurotoxicity of substituted amphetamines: molecular and cellular mechanisms. Neurotox Res 11, 183-202.

Cadet, J.L., McCoy, M.T., Ladenheim, B., 2002. Distinct gene expression signatures in the striata of wildtype and heterozygous c-fos knockout mice following methamphetamine administration: evidence from cDNA array analyses. Synapse 44, 211-226.

Callaghan, R.C., Cunningham, J.K., Sajeev, G., Kish, S.J., 2010. Incidence of Parkinson's disease among hospital patients with methamphetamine-use disorders. Mov Disord 25, 2333-2339.

Callaghan, R.C., Cunningham, J.K., Sykes, J., Kish, S.J., 2012. Increased risk of Parkinson's disease in individuals hospitalized with conditions related to the use of methamphetamine or other amphetaminetype drugs. Drug Alcohol Depend 120, 35-40.

Cantarella, G., Bucolo, C., Di Benedetto, G., Pezzino, S., Lempereur, L., Calvagna, R., Clementi, S., Pavone, P., Fiore, L., Bernardini, R., 2007. Protective effects of the sigma agonist Pre-084 in the rat retina. Br J Ophthalmol 91, 1382-1384. 
Cassano, G., Gasparre, G., Niso, M., Contino, M., Scalera, V., Colabufo, N.A., 2009. F281, synthetic agonist of the sigma-2 receptor, induces $\mathrm{Ca} 2+$ efflux from the endoplasmic reticulum and mitochondria in SK-N-SH cells. Cell Calcium 45, 340-345.

Castren, E., Rantamaki, T., 2010. The role of BDNF and its receptors in depression and antidepressant drug action: Reactivation of developmental plasticity. Dev Neurobiol 70, 289-297.

Chaput, Y., de Montigny, C., Blier, P., 1991. Presynaptic and postsynaptic modifications of the serotonin system by long-term administration of antidepressant treatments. An in vivo electrophysiologic study in the rat. Neuropsychopharmacology 5, 219-229.

Chen, J.C., Chen, P.C., Chiang, Y.C., 2009. Molecular mechanisms of psychostimulant addiction. Chang Gung Med J 32, 148-154.

Chen, S.H., Benveniste, E.N., 2004. Oncostatin M: a pleiotropic cytokine in the central nervous system. Cytokine Growth Factor Rev 15, 379-391.

Chen, S.H., Gillespie, G.Y., Benveniste, E.N., 2006. Divergent effects of oncostatin M on astroglioma cells: influence on cell proliferation, invasion, and expression of matrix metalloproteinases. Glia 53, 191-200.

Chen, X.S., S. Bayliss, DA., 2009. HCN1 chnnel subunits are a molecular substrate for hypnotic actions of ketmine. J Neurosci 29, 600-609.

Cheng, Y., Prusoff, W.H., 1973. Relationship between the inhibition constant (K1) and the concentration of inhibitor which causes 50 per cent inhibition (150) of an enzymatic reaction. Biochem Pharmacol 22, 3099-3108.

Chipana, C., Torres, I., Camarasa, J., Pubill, D., Escubedo, E., 2008. Memantine protects against amphetamine derivatives-induced neurotoxic damage in rodents. Neuropharmacology 54, 1254-1263. Cooper, Z.D., Jones, J.D., Comer, S.D., 2012. Glial modulators: a novel pharmacological approach to altering the behavioral effects of abused substances. Expert Opin Investig Drugs 21, 169-178.

Coulter, D.A., Eid, T., 2012. Astrocytic regulation of glutamate homeostasis in epilepsy. Glia 60, 12151226.

Crawford, K.W., Bowen, W.D., 2002. Sigma-2 receptor agonists activate a novel apoptotic pathway and potentiate antineoplastic drugs in breast tumor cell lines. Cancer Res 62, 313-322.

Cuevas, J., Rodriguez, A., Behensky, A., Katnik, C., 2011. Afobazole modulates microglial function via activation of both sigma-1 and sigma-2 receptors. J Pharmacol Exp Ther 339, 161-172.

Czeh, M., Gressens, P., Kaindl, A.M., 2011. The yin and yang of microglia. Dev Neurosci 33, 199-209. de Costa, B.R., Bowen, W.D., Hellewell, S.B., Walker, J.M., Thurkauf, A., Jacobson, A.E., Rice, K.C., 1989. Synthesis and evaluation of optically pure $[3 \mathrm{H}]-(+)$-pentazocine, a highly potent and selective radioligand for sigma receptors. FEBS Lett 251, 53-58.

de Costa, B.R., Radesca, L., Di Paolo, L., Bowen, W.D., 1992. Synthesis, characterization, and biological evaluation of a novel class of $\mathrm{N}$-(arylethyl)- $\mathrm{N}$-alkyl-2-(1-pyrrolidinyl)ethylamines: structural requirements and binding affinity at the sigma receptor. J Med Chem 35, 38-47.

Deng, X., Wang, Y., Chou, J., Cadet, J.L., 2001. Methamphetamine causes widespread apoptosis in the mouse brain: evidence from using an improved TUNEL histochemical method. Brain Res Mol Brain Res 93, 64-69.

Derocq, J.M., Bourrie, B., Segui, M., Le Fur, G., Casellas, P., 1995. In vivo inhibition of endotoxin-induced pro-inflammatory cytokines production by the sigma ligand SR 31747. J Pharmacol Exp Ther 272, 224230.

Deverteuil, R.L., Lehmann, H.E., 1958. Therapeutic trial of iproniazid (marsilid) in depressed and apathetic patients. Can Med Assoc J 78, 131-133.

Diazgranados, N., Ibrahim, L., Brutsche, N.E., Newberg, A., Kronstein, P., Khalife, S., Kammerer, W.A., Quezado, Z., Luckenbaugh, D.A., Salvadore, G., Machado-Vieira, R., Manji, H.K., Zarate, C.A., Jr., 2010a. A randomized add-on trial of an $\mathrm{N}$-methyl-D-aspartate antagonist in treatment-resistant bipolar depression. Arch Gen Psychiatry 67, 793-802. 
Diazgranados, N., Ibrahim, L.A., Brutsche, N.E., Ameli, R., Henter, I.D., Luckenbaugh, D.A., MachadoVieira, R., Zarate, C.A., Jr., 2010b. Rapid resolution of suicidal ideation after a single infusion of an Nmethyl-D-aspartate antagonist in patients with treatment-resistant major depressive disorder. J Clin Psychiatry.

Duman, R.S., Heninger, G.R., Nestler, E.J., 1997. A molecular and cellular theory of depression. Arch Gen Psychiatry 54, 597-606.

Duman, R.S., Malberg, J., Thome, J., 1999. Neural plasticity to stress and antidepressant treatment. Biol Psychiatry 46, 1181-1191.

Duman, R.S., Nakagawa, S., Malberg, J., 2001. Regulation of adult neurogenesis by antidepressant treatment. Neuropsychopharmacology 25, 836-844.

Ehmke, H., 2012. The sigma-1 receptor: a molecular chaperone for the heart and the soul? Cardiovasc Res 93, 6-7.

Eisch, A.J., Schmued, L.C., Marshall, J.F., 1998. Characterizing cortical neuron injury with Fluoro-Jade labeling after a neurotoxic regimen of methamphetamine. Synapse 30, 329-333.

Engin, E., Treit, D., Dickson, C.T., 2009. Anxiolytic- and antidepressant-like properties of ketamine in behavioral and neurophysiological animal models. Neuroscience 161, 359-369.

Escubedo, E., Camarasa, J., Chipana, C., Garcia-Rates, S., Pubill, D., 2009. Involvement of nicotinic receptors in methamphetamine- and MDMA-induced neurotoxicity: pharmacological implications. Int Rev Neurobiol 88, 121-166.

Escubedo, E., Chipana, C., Perez-Sanchez, M., Camarasa, J., Pubill, D., 2005. Methyllycaconitine prevents methamphetamine-induced effects in mouse striatum: involvement of alpha7 nicotinic receptors. J

Pharmacol Exp Ther 315, 658-667.

Escubedo, E., Guitart, L., Sureda, F.X., Jimenez, A., Pubill, D., Pallas, M., Camins, A., Camarasa, J., 1998.

Microgliosis and down-regulation of adenosine transporter induced by methamphetamine in rats. Brain Res 814, 120-126.

Fava, M., Davidson, K.G., 1996. Definition and epidemiology of treatment-resistant depression. Psychiatr Clin North Am 19, 179-200.

Fishback, J.A., Robson, M.J., Xu, Y.-T., Matsumoto, R.R., 2010a. Sigma receptors: Potential targets for a new class of antidepressant drug. Pharmacology and Therapeutics 127, 271-282.

Fishback, J.A., Robson, M.J., Xu, Y.T., Matsumoto, R.R., 2010b. Sigma receptors: potential targets for a new class of antidepressant drug. Pharmacol Ther 127, 271-282.

Fontanilla, D., Johannessen, M., Hajipour, A.R., Cozzi, N.V., Jackson, M.B., Ruoho, A.E., 2009. The hallucinogen $\mathrm{N}, \mathrm{N}$-dimethyltryptamine (DMT) is an endogenous sigma-1 receptor regulator. Science 323, 934-937.

Frazer, A., Benmansour, S., 2002. Delayed pharmacological effects of antidepressants. Mol Psychiatry 7 Suppl 1, S23-28.

Galehdar, Z., Swan, P., Fuerth, B., Callaghan, S.M., Park, D.S., Cregan, S.P., 2010. Neuronal apoptosis induced by endoplasmic reticulum stress is regulated by ATF4-CHOP-mediated induction of the Bcl-2 homology 3-only member PUMA. J Neurosci 30, 16938-16948.

Gebreselassie, D., Bowen, W.D., 2004. Sigma-2 receptors are specifically localized to lipid rafts in rat liver membranes. Eur J Pharmacol 493, 19-28.

Goncalves, J., Martins, T., Ferreira, R., Milhazes, N., Borges, F., Ribeiro, C.F., Malva, J.O., Macedo, T.R., Silva, A.P., 2008. Methamphetamine-induced early increase of IL-6 and TNF-alpha mRNA expression in the mouse brain. Ann N Y Acad Sci 1139, 103-111.

Gonzalez-Alvear, G.M., Werling, L.L., 1994. Regulation of [3H]dopamine release from rat striatal slices by sigma receptor ligands. J Pharmacol Exp Ther 271, 212-219.

Gonzalez-Alvear, G.M., Werling, L.L., 1995. Sigma1 Receptors in rat striatum regulate NMDA-stimulated [3H]dopamine release via a presynaptic mechanism. Eur J Pharmacol 294, 713-719. 
Gonzalez, G.M., Werling, L.L., 1997. Release of [3H]dopamine from guinea pig striatal slices is modulated by sigma1 receptor agonists. Naunyn Schmiedebergs Arch Pharmacol 356, 455-461.

Gonzalez, R., Bechara, A., Martin, E.M., 2007. Executive functions among individuals with methamphetamine or alcohol as drugs of choice: preliminary observations. J Clin Exp Neuropsychol 29, 155-159.

Gonzalez, R., Rippeth, J.D., Carey, C.L., Heaton, R.K., Moore, D.J., Schweinsburg, B.C., Cherner, M., Grant, I., 2004. Neurocognitive performance of methamphetamine users discordant for history of marijuana exposure. Drug Alcohol Depend 76, 181-190.

Gorman, A.M., Healy, S.J., Jager, R., Samali, A., 2012. Stress management at the ER: regulators of ER stress-induced apoptosis. Pharmacol Ther 134, 306-316.

Gross, N.B., Duncker, P.C., Marshall, J.F., 2011. Striatal dopamine D1 and D2 receptors: widespread influences on methamphetamine-induced dopamine and serotonin neurotoxicity. Synapse 65, 11441155.

Gudelsky, G.A., 1995. Effects of sigma receptor ligands on the extracellular concentration of dopamine in the striatum and prefrontal cortex of the rat. Eur J Pharmacol 286, 223-228.

Guilarte, T.R., 2001. Is methamphetamine abuse a risk factor in parkinsonism? Neurotoxicology 22, 725731.

Hall, A.A., Herrera, Y., Ajmo, C.T., Jr., Cuevas, J., Pennypacker, K.R., 2009. Sigma receptors suppress multiple aspects of microglial activation. Glia 57, 744-754.

Hart, C.L., Marvin, C.B., Silver, R., Smith, E.E., 2012. Is cognitive functioning impaired in methamphetamine users? A critical review. Neuropsychopharmacology 37, 586-608.

Hashimoto, K., Fujita, Y., lyo, M., 2007a. Phencyclidine-induced cognitive deficits in mice are improved by subsequent subchronic administration of fluvoxamine: role of sigma-1 receptors.

Neuropsychopharmacology 32, 514-521.

Hashimoto, K., Tsukada, H., Nishiyama, S., Fukumoto, D., Kakiuchi, T., lyo, M., 2007b. Protective effects of minocycline on the reduction of dopamine transporters in the striatum after administration of methamphetamine: a positron emission tomography study in conscious monkeys. Biol Psychiatry 61, 577-581.

Hayashi, T., Justinova, Z., Hayashi, E., Cormaci, G., Mori, T., Tsai, S.Y., Barnes, C., Goldberg, S.R., Su, T.P., 2010. Regulation of sigma-1 receptors and endoplasmic reticulum chaperones in the brain of methamphetamine self-administering rats. J Pharmacol Exp Ther 332, 1054-1063.

Hayashi, T., Maurice, T., Su, T.P., 2000a. Ca(2+) signaling via sigma(1)-receptors: novel regulatory mechanism affecting intracellular $\mathrm{Ca}(2+)$ concentration. J Pharmacol Exp Ther 293, 788-798.

Hayashi, T., Maurice, T., Su, T.P., 2000b. Ca(2+) signaling via sigma(1)-receptors: novel regulatory mechanism affecting intracellular $\mathrm{Ca}(2+)$ concentration. J Pharmacol Exp Ther 293, 788-798.

Hayashi, T., Rizzuto, R., Hajnoczky, G., Su, T.P., 2009. MAM: more than just a housekeeper. Trends Cell Biol 19, 81-88.

Hayashi, T., Su, T.P., 2001. Regulating ankyrin dynamics: Roles of sigma-1 receptors. Proc Natl Acad Sci U S A 98, 491-496.

Hayashi, T., Su, T.P., 2003. Intracellular dynamics of sigma-1 receptors (sigma(1) binding sites) in NG10815 cells. J Pharmacol Exp Ther 306, 726-733.

Hayashi, T., Su, T.P., 2005. The potential role of sigma-1 receptors in lipid transport and lipid raft reconstitution in the brain: implication for drug abuse. Life Sci 77, 1612-1624.

Hayashi, T., Su, T.P., 2007a. Sigma-1 receptor chaperones at the ER-mitochondrion interface regulate $\mathrm{Ca}(2+)$ signaling and cell survival. 131, 596-610.

Hayashi, T., Su, T.P., 2007b. Sigma-1 receptor chaperones at the ER-mitochondrion interface regulate $\mathrm{Ca}(2+)$ signaling and cell survival. Cell 131, 596-610. 
Hayashi, T., Su, T.P., 2008. An update on the development of drugs for neuropsychiatric disorders: focusing on the sigma 1 receptor ligand. Expert Opin Ther Targets 12, 45-58.

Hayashi, T., Tsai, S.Y., Mori, T., Fujimoto, M., Su, T.P., 2011. Targeting ligand-operated chaperone sigma1 receptors in the treatment of neuropsychiatric disorders. Expert Opin Ther Targets 15, 557-577. Hebert, M.A., O'Callaghan, J.P., 2000. Protein phosphorylation cascades associated with methamphetamine-induced glial activation. Ann N Y Acad Sci 914, 238-262.

Heinrich, P.C., Behrmann, I., Haan, S., Hermanns, H.M., Muller-Newen, G., Schaper, F., 2003. Principles of interleukin (IL)-6-type cytokine signalling and its regulation. Biochem J 374, 1-20.

Hellewell, S.B., Bowen, W.D., 1990. A sigma-like binding site in rat pheochromocytoma (PC12) cells: decreased affinity for (+)-benzomorphans and lower molecular weight suggest a different sigma receptor form from that of guinea pig brain. Brain Res 527, 244-253.

Hellewell, S.B., Bruce, A., Feinstein, G., Orringer, J., Williams, W., Bowen, W.D., 1994. Rat liver and kidney contain high densities of sigma 1 and sigma 2 receptors: characterization by ligand binding and photoaffinity labeling. Eur J Pharmacol 268, 9-18.

Hirota, K., Sikand, K.S., Lambert, D.G., 1999. Interaction of ketamine with mu2 opioid receptors in SHSY5Y human neuroblastoma cells. J Anesth 13, 107-109.

Hustveit, O., Maurset, A., Oye, I., 1995. Interaction of chiral forms of ketamine with opiod, phencyclidine, sigma and muscarinic receptors. Pharmacology and Toxicology 77, 355-359. Imam, S.Z., Itzhak, Y., Cadet, J.L., Islam, F., Slikker, W., Jr., Ali, S.F., 2001. Methamphetamine-induced alteration in striatal p53 and bcl-2 expressions in mice. Brain Res Mol Brain Res 91, 174-178. Irie, Y., Saeki, M., Tanaka, H., Kanemura, Y., Otake, S., Ozono, Y., Nagai, T., Kondo, Y., Kudo, K., Kamisaki, Y., Miki, N., Taira, E., 2011. Methamphetamine induces endoplasmic reticulum stress related gene CHOP/Gadd153/ddit3 in dopaminergic cells. Cell Tissue Res 345, 231-241.

Ishima, T., Nishimura, T., Iyo, M., Hashimoto, K., 2008. Potentiation of nerve growth factor-induced neurite outgrowth in PC12 cells by donepezil: role of sigma-1 receptors and IP3 receptors. Prog Neuropsychopharmacol Biol Psychiatry 32, 1656-1659.

Itzhak, Y., 1993. Repeated methamphetamine-treatment alters brain sigma receptors. Eur J Pharmacol 230, 243-244.

Itzhak, Y., Kassim, C.O., 1990. Clorgyline displays high affinity for sigma binding sites in C57BL/6 mouse brain. Eur J Pharmacol 176, 107-108.

Iwamoto, E.T., 1981. Locomotor activity and antinociception after putative mu, kappa and sigma opioid receptor agonists in the rat: influence of dopaminergic agonists and antagonists. J Pharmacol Exp Ther 217, 451-460.

lyengar, S., Dilworth, V.M., Mick, S.J., Contreras, P.C., Monahan, J.B., Rao, T.S., Wood, P.L., 1990. Sigma receptors modulate both $\mathrm{A} 9$ and $\mathrm{A} 10$ dopaminergic neurons in the rat brain: functional interaction with NMDA receptors. Brain Res 524, 322-326.

Jayanthi, S., Deng, X., Bordelon, M., McCoy, M.T., Cadet, J.L., 2001. Methamphetamine causes differential regulation of pro-death and anti-death $\mathrm{Bcl}-2$ genes in the mouse neocortex. FASEB J 15 , 1745-1752.

Jayanthi, S., Deng, X., Ladenheim, B., McCoy, M.T., Cluster, A., Cai, N.S., Cadet, J.L., 2005. Calcineurin/NFAT-induced up-regulation of the Fas ligand/Fas death pathway is involved in methamphetamine-induced neuronal apoptosis. Proc Natl Acad Sci U S A 102, 868-873.

Jayanthi, S., Deng, X., Noailles, P.A., Ladenheim, B., Cadet, J.L., 2004. Methamphetamine induces neuronal apoptosis via cross-talks between endoplasmic reticulum and mitochondria-dependent death cascades. FASEB J 18, 238-251.

Jayanthi, S., McCoy, M.T., Beauvais, G., Ladenheim, B., Gilmore, K., Wood, W., 3rd, Becker, K., Cadet, J.L., 2009. Methamphetamine induces dopamine D1 receptor-dependent endoplasmic reticulum stressrelated molecular events in the rat striatum. PLoS One 4, e6092. 
Jupp, B., Lawrence, A.J., 2010. New horizons for therapeutics in drug and alcohol abuse. Pharmacol Ther 125, 138-168.

Kahoun, J.R., Ruoho, A.E., 1992. (125I)iodoazidococaine, a photoaffinity label for the haloperidolsensitive sigma receptor. Proc Natl Acad Sci U S A 89, 1393-1397.

Kalivas, P.W., Nakamura, M., 1999. Neural systems for behavioral activation and reward. Curr Opin Neurobiol 9, 223-227.

Kaushal, N., Elliott, M., Robson, M.J., Iyer, A.K., Rojanasakul, Y., Coop, A., Matsumoto, R.R., 2011 a. AC927, a sigma ligand, blocks methamphetamine-induced release of dopamine and generation of reactive oxygen species in NG108-15 cells. Mol Pharmacol.

Kaushal, N., Elliott, M., Robson, M.J., Iyer, A.K., Rojanasakul, Y., Coop, A., Matsumoto, R.R., 2012a. AC927, a sigma receptor ligand, blocks methamphetamine-induced release of dopamine and generation of reactive oxygen species in NG108-15 cells. Mol Pharmacol 81, 299-308.

Kaushal, N., Matsumoto, R.R., 2011. Role of sigma receptors in methamphetamine-induced neurotoxicity. Curr Neuropharmacol 9, 54-57.

Kaushal, N., McCurdy, C.R., Matsumoto, R.R., 2011b. SN79 attenuates the neurotoxic effect of methamphetamine: In vivo and in vitro studies. Society for Neuroscience 2011 Neuroscience Meeting Planner.

Kaushal, N., McCurdy, C.R., Matsumoto, R.R., 2011c. SN79 attenuates the neurotoxic effect of methamphetamine: in vivo and in vitro studies. 2011 Meeting Planner. Washington, D.C: Society for Neuroscience 367.03.

Kaushal, N., McCurdy, C.R., Matsumoto, R.R., 2011d. SN79 attenuates the neurotoxic effects of methamphetamine: in vivo and in vitro studies. Society for Neuroscience Abstract.

Kaushal, N., Robson, M.J., Vinnakota, H., Narayanan, S., Avery, B.A., McCurdy, C.R., Matsumoto, R.R., 2011e. Synthesis and pharmacological evaluation of 6-acetyl-3-(4-(4-(4-fluorophenyl)piperazin-1yl)butyl)benzo[d]oxazol-2(3H)-one (SN79), a cocaine antagonist, in rodents. AAPS J 13, 336-346.

Kaushal, N., Seminerio, M.J., Robson, M.J., McCurdy, C.R., Matsumoto, R.R., 2012b. Pharmacological evaluation of SN79, a sigma (sigma) receptor ligand, against methamphetamine-induced neurotoxicity in vivo. Eur Neuropsychopharmacol.

Kaushal, N., Seminerio, M.J., Shaikh, J., Medina, M.A., Mesangeau, C., Wilson, L.L., McCurdy, C.R., Matsumoto, R.R., 2011f. CM156, a high affinity sigma ligand, attenuates the stimulant and neurotoxic effects of methamphetamine in mice. Neuropharmacology 61, 992-1000.

Kavalali, E.T., Monteggia, L.M., 2012. Synaptic Mechanisms Underlying Rapid Antidepressant Action of Ketamine. Am J Psychiatry.

Kekuda, R., Prasad, P.D., Fei, Y.J., Leibach, F.H., Ganapathy, V., 1996. Cloning and functional expression of the human type 1 sigma receptor (hSigmaR1). Biochem Biophys Res Commun 229, 553-558.

Kelly, K.A., Miller, D.B., Bowyer, J.F., O'Callaghan, J.P., 2012. Chronic exposure to corticosterone enhances the neuroinflammatory and neurotoxic responses to methamphetamine. J Neurochem 122, 995-1009.

Kessler, R.C., Merikangas, K.R., Wang, P.S., 2007. Prevalence, comorbidity, and service utilization for mood disorders in the United States at the beginning of the twenty-first century. Annu Rev Clin Psychol 3, 137-158.

Kishimoto, A.T., A. Miura, J. Kitagaki, T. Hashimoto, K., 2010. The opposite effects of fluvoxmine and sertraline in the treatment of psychotic major depression: a case report. Ann Gen Psychiatry 9, 23.

Kita, T., Shimada, K., Mastunari, Y., Wagner, G.C., Kubo, K., Nakashima, T., 2000. Methamphetamineinduced striatal dopamine neurotoxicity and cyclooxygenase-2 protein expression in BALB/c mice. Neuropharmacology 39, 399-406.

Kiyatkin, E.A., Sharma, H.S., 2009. Acute methamphetamine intoxication: brain hyperthermia, bloodbrain barrier, brain edema, and morphological cell abnormalities. Int Rev Neurobiol 88, 65-100. 
Klouz, A., Tillement, J.P., Boussard, M.F., Wierzbicki, M., Berezowski, V., Cecchelli, R., Labidalle, S., Onteniente, B., Morin, D., 2003. [3H]BHDP as a novel and selective ligand for sigma1 receptors in liver mitochondria and brain synaptosomes of the rat. FEBS Lett 553, 157-162.

Kobayashi, T., Matsuno, K., Murai, M., Mita, S., 1997. Sigma 1 receptor subtype is involved in the facilitation of cortical dopaminergic transmission in the rat brain. Neurochem Res 22, 1105-1109. Koike, H., lijima, M., Chaki, S., 2011. Involvement of AMPA receptor in both the rapid and sustained antidepressant-like effects of ketamine in animal models of depression. Behav Brain Res 224, 107-111. Kollmar, R., Markovic, K., Thurauf, N., Schmitt, H., Kornhuber, J., 2008. Ketamine followed by memantine for the treatment of major depression. Aust N Z J Psychiatry 42, 170.

Komohara, Y., Horlad, H., Ohnishi, K., Ohta, K., Makino, K., Hondo, H., Yamanaka, R., Kajiwara, K., Saito, T., Kuratsu, J., Takeya, M., 2011. M2 macrophage/microglial cells induce activation of Stat3 in primary central nervous system lymphoma. J Clin Exp Hematop 51, 93-99.

Kortekaas, R., Maguire, R.P., van Waarde, A., Leenders, K.L., Elsinga, P.H., 2008. Despite irreversible binding, PET tracer [11C]-SA5845 is suitable for imaging of drug competition at sigma receptors-the cases of ketamine and haloperidol. Neurochem Int 53, 45-50.

Kourrich, S., Hayashi, T., Chuang, J.Y., Tsai, S.Y., Su, T.P., Bonci, A., 2013. Dynamic interaction between sigma-1 receptor and Kv1.2 shapes neuronal and behavioral responses to cocaine. Cell 152, 236-247. Kourrich, S., Su, T.P., Fujimoto, M., Bonci, A., 2012. The sigma-1 receptor: roles in neuronal plasticity and disease. Trends Neurosci 35, 762-771.

Krasnova, I.N., Cadet, J.L., 2009. Methamphetamine toxicity and messengers of death. Brain Res Rev 60, 379-407.

Krasnova, I.N., Justinova, Z., Ladenheim, B., Jayanthi, S., McCoy, M.T., Barnes, C., Warner, J.E., Goldberg, S.R., Cadet, J.L., 2010. Methamphetamine self-administration is associated with persistent biochemical alterations in striatal and cortical dopaminergic terminals in the rat. PLoS One 5, e8790.

Krystal, J.H., 2007. Ketamine and the potential role for rapid-acting antidepressant medications. Swiss Med Wkly 137, 215-216.

Kuehn, B.M., 2011. Meth use linked to risk of Parkinson disease. JAMA 306, 814.

Kuhn, D.M., Francescutti-Verbeem, D.M., Thomas, D.M., 2006. Dopamine quinones activate microglia and induce a neurotoxic gene expression profile: relationship to methamphetamine-induced nerve ending damage. Ann N Y Acad Sci 1074, 31-41.

Kulkarni, S.K., Dhir, A., 2009a. Current investigational drugs for major depression. Expert Opin Investig Drugs 18, 767-788.

Kulkarni, S.K., Dhir, A., 2009b. Current investigational drugs for major depression. Expert Opin Investig Drugs.

Kulkarni, S.K., Dhir, A., 2009c. Sigma-1 receptors in major depression and anxiety. Expert Rev Neurother 9, 1021-1034.

Ladenheim, B., Krasnova, I.N., Deng, X., Oyler, J.M., Polettini, A., Moran, T.H., Huestis, M.A., Cadet, J.L., 2000. Methamphetamine-induced neurotoxicity is attenuated in transgenic mice with a null mutation for interleukin-6. Mol Pharmacol 58, 1247-1256.

Lai, E., Teodoro, T., Volchuk, A., 2007. Endoplasmic reticulum stress: signaling the unfolded protein response. Physiology 22, 193-201.

Larkin, G.L., Smith, R.P., Beautrais, A.L., 2008. Trends in US emergency department visits for suicide attempts, 1992-2001. Crisis 29, 73-80.

Lau, J.W., Senok, S., Stadlin, A., 2000. Methamphetamine-induced oxidative stress in cultured mouse astrocytes. Ann N Y Acad Sci 914, 146-156.

Lauterbach, E., 2011. Dextromethorphan as a potential rapid-acting antidepressant. Medical Hypotheses Article in Press. 
LaVoie, M.J., Card, J.P., Hastings, T.G., 2004. Microglial activation precedes dopamine terminal pathology in methamphetamine-induced neurotoxicity. Exp Neurol 187, 47-57.

LaVoie, M.J., Hastings, T.G., 1999a. Dopamine quinone formation and protein modification associated with the striatal neurotoxicity of methamphetamine: evidence against a role for extracellular dopamine. J Neurosci 19, 1484-1491.

LaVoie, M.J., Hastings, T.G., 1999b. Peroxynitrite- and nitrite-induced oxidation of dopamine: implications for nitric oxide in dopaminergic cell loss. J Neurochem 73, 2546-2554.

Li, B., Mahmood, A., Lu, D., Wu, H., Xiong, Y., Qu, C., Chopp, M., 2009. Simvastatin attenuates microglial cells and astrocyte activation and decreases interleukin-1beta level after traumatic brain injury.

Neurosurgery 65, 179-185; discussion 185-176.

Li, L., Xu, B., Zhu, Y., Chen, L., Sokabe, M., 2010a. DHEA prevents Abeta(25-35)-impaired survival of newborn neurons in the dentate gyrus through a modulation of PI3K-Akt-mTOR signaling.

Neuropharmacology 59, 323-333.

Li, N., Lee, B., Liu, R.J., Banasr, M., Dwyer, J.M., Iwata, M., Li, X.Y., Aghajanian, G., Duman, R.S., $2010 b$. mTOR-dependent synapse formation underlies the rapid antidepressant effects of NMDA antagonists. Science 329, 959-964.

Li, N., Liu, R.J., Dwyer, J.M., Banasr, M., Lee, B., Son, H., Li, X.Y., Aghajanian, G., Duman, R.S., 2011. Glutamate N-methyl-D-aspartate receptor antagonists rapidly reverse behavioral and synaptic deficits caused by chronic stress exposure. Biol Psychiatry 69, 754-761.

Lin, J.H., Li, H., Yasumura, D., Cohen, H.R., Zhang, C., Panning, B., Shokat, K.M., Lavail, M.M., Walter, P., 2007. IRE1 signaling affects cell fate during the unfolded protein response. Science 318, 944-949.

Liu, X., Nuwayhid, S., Christie, M.J., Kassiou, M., Werling, L.L., 2001. Trishomocubanes: novel sigmareceptor ligands modulate amphetamine-stimulated [3H]dopamine release. Eur J Pharmacol 422, 39-45. Liu, Y., Chen, G.D., Lerner, M.R., Brackett, D.J., Matsumoto, R.R., 2005. Cocaine up-regulates Fra-2 and sigma-1 receptor gene and protein expression in brain regions involved in addiction and reward. J Pharmacol Exp Ther 314, 770-779.

Liu, Y., Matsumoto, R.R., 2008. Alterations in fos-related antigen 2 and sigma1 receptor gene and protein expression are associated with the development of cocaine-induced behavioral sensitization: time course and regional distribution studies. J Pharmacol Exp Ther 327, 187-195.

Machado-Vieira, R., Salvadore, G., Diazgranados, N., Zarate, C.A., Jr., 2009. Ketamine and the next generation of antidepressants with a rapid onset of action. Pharmacol Ther 123, 143-150.

Maeda, D.Y., Williams, W., Kim, W.E., Thatcher, L.N., Bowen, W.D., Coop, A., 2002. N-arylalkylpiperidines as high-affinity sigma-1 and sigma-2 receptor ligands: phenylpropylamines as potential leads for selective sigma-2 agents. Bioorg Med Chem Lett 12, 497-500.

Maeng, S., Zarate, C.A., Jr., 2007. The role of glutamate in mood disorders: results from the ketamine in major depression study and the presumed cellular mechanism underlying its antidepressant effects.

Curr Psychiatry Rep 9, 467-474.

Maeng, S., Zarate, C.A., Jr., Du, J., Schloesser, R.J., McCammon, J., Chen, G., Manji, H.K., 2008. Cellular mechanisms underlying the antidepressant effects of ketamine: role of alpha-amino-3-hydroxy-5methylisoxazole-4-propionic acid receptors. Biol Psychiatry 63, 349-352.

Marciniak, S.J., Yun, C.Y., Oyadomari, S., Novoa, I., Zhang, Y., Jungreis, R., Nagata, K., Harding, H.P., Ron, D., 2004. CHOP induces death by promoting protein synthesis and oxidation in the stressed endoplasmic reticulum. Genes Dev 18, 3066-3077.

Martin, A.L., Brown, R.E., 2010. The lonely mouse: verification of a separation-induced model of depression in female mice. Behav Brain Res 207, 196-207.

Martin, W.R., Eades, C.G., Thompson, J.A., Huppler, R.E., Gilbert, P.E., 1976. The effects of morphineand nalorphine- like drugs in the nondependent and morphine-dependent chronic spinal dog. J Pharmacol Exp Ther 197, 517-532. 
Martina, M., Turcotte, M.E., Halman, S., Bergeron, R., 2007. The sigma-1 receptor modulates NMDA receptor synaptic transmission and plasticity via SK channels in rat hippocampus. J Physiol 578, 143-157. Matsumoto, R.R., 2009. Targeting sigma receptors: novel medication development for drug abuse and addiction. Expert Rev Clin Pharmacol 2, 351-358.

Matsumoto, R.R., Bowen, W.D., Su, T.P., 2007. Sigma receptors: chemistry, cell biology and clinical implications. Springer, New York.

Matsumoto, R.R., Hewett, K.L., Pouw, B., Bowen, W.D., Husbands, S.M., Cao, J.J., Hauck Newman, A., 2001a. Rimcazole analogs attenuate the convulsive effects of cocaine: correlation with binding to sigma receptors rather than dopamine transporters. Neuropharmacology 41, 878-886.

Matsumoto, R.R., McCracken, K.A., Friedman, M.J., Pouw, B., De Costa, B.R., Bowen, W.D., 2001b.

Conformationally restricted analogs of BD1008 and an antisense oligodeoxynucleotide targeting sigma1 receptors produce anti-cocaine effects in mice. Eur J Pharmacol 419, 163-174.

Matsumoto, R.R., Shaikh, J., Wilson, L.L., Vedam, S., Coop, A., 2008. Attenuation of methamphetamineinduced effects through the antagonism of sigma (sigma) receptors: Evidence from in vivo and in vitro studies. Eur Neuropsychopharmacol 18, 871-881.

Matsuno, K., Kobayashi, T., Tanaka, M.K., Mita, S., 1996. Sigma 1 receptor subtype is involved in the relief of behavioral despair in the mouse forced swimming test. Eur J Pharmacol 312, 267-271.

McCracken, K.A., Bowen, W.D., de Costa, B.R., Matsumoto, R.R., 1999. Two novel sigma receptor ligands, BD1047 and LR172, attenuate cocaine-induced toxicity and locomotor activity. Eur J Pharmacol 370, 225-232.

McCullough, K.D., Martindale, J.L., Klotz, L.O., Aw, T.Y., Holbrook, N.J., 2001. Gadd153 sensitizes cells to endoplasmic reticulum stress by down-regulating $\mathrm{Bcl} 2$ and perturbing the cellular redox state. Mol Cell Biol 21, 1249-1259.

Mei, J., Pasternak, G.W., 2001. Molecular cloning and pharmacological characterization of the rat sigma1 receptor. Biochem Pharmacol 62, 349-355.

Mendelsohn, L.G., Kalra, V., Johnson, B.G., Kerchner, G.A., 1985. Sigma opioid receptor: characterization and co-identity with the phencyclidine receptor. J Pharmacol Exp Ther 233, 597-602.

Meunier, J., Hayashi, T., 2010. Sigma-1 receptors regulate Bcl-2 expression by reactive oxygen speciesdependent transcriptional regulation of nuclear factor kappaB. J Pharmacol Exp Ther 332, 388-397.

Miguel-Hidalgo, J.J., 2009. The role of glial cells in drug abuse. Curr Drug Abuse Rev 2, 72-82.

Miyazaki, I., Asanuma, M., 2009. Approaches to prevent dopamine quinone-induced neurotoxicity. Neurochem Res 34, 698-706.

Mizoguchi, H., Takuma, K., Fukakusa, A., Ito, Y., Nakatani, A., Ibi, D., Kim, H.C., Yamada, K., 2008.

Improvement by minocycline of methamphetamine-induced impairment of recognition memory in mice. Psychopharmacology (Berl) 196, 233-241.

Monnet, F.P., Blier, P., Debonnel, G., de Montigny, C., 1992a. Modulation by sigma ligands of N-methyl$\mathrm{D}$-aspartate-induced $[3 \mathrm{H}]$ noradrenaline release in the rat hippocampus: G-protein dependency. Naunyn Schmiedebergs Arch Pharmacol 346, 32-39.

Monnet, F.P., de Costa, B.R., Bowen, W.D., 1996. Differentiation of sigma ligand-activated receptor subtypes that modulate NMDA-evoked [3H]-noradrenaline release in rat hippocampal slices. $\mathrm{Br} \mathrm{J}$ Pharmacol 119, 65-72.

Monnet, F.P., Debonnel, G., de Montigny, C., 1992b. In vivo electrophysiological evidence for a selective modulation of $\mathrm{N}$-methyl-D-aspartate-induced neuronal activation in rat CA3 dorsal hippocampus by sigma ligands. J Pharmacol Exp Ther 261, 123-130.

Monnet, F.P., Debonnel, G., Junien, J.L., De Montigny, C., 1990. N-methyl-D-aspartate-induced neuronal activation is selectively modulated by sigma receptors. Eur J Pharmacol 179, 441-445. 
Moon, J.Y., Roh, D.H., Yoon, S.Y., Kang, S.Y., Choi, S.R., Kwon, S.G., Choi, H.S., Han, H.J., Beitz, A.J., Lee, J.H., 2013. Sigma-1 receptor-mediated increase in spinal p38 MAPK phosphorylation leads to the induction of mechanical allodynia in mice and neuropathic rats. Exp Neurol.

Mosser, D.M., Edwards, J.P., 2008. Exploring the full spectrum of macrophage activation. Nat Rev Immunol 8, 958-969.

Murrough, J.W., Charney, D.S., 2012. Is there anything really novel on the antidepressant horizon? Curr Psychiatry Rep 14, 643-649.

Nagai, A., Mishima, S., Ishida, Y., Ishikura, H., Harada, T., Kobayashi, S., Kim, S.U., 2005. Immortalized human microglial cell line: phenotypic expression. J Neurosci Res 81, 342-348.

Nakajima, A., Yamada, K., Nagai, T., Uchiyama, T., Miyamoto, Y., Mamiya, T., He, J., Nitta, A., Mizuno, M., Tran, M.H., Seto, A., Yoshimura, M., Kitaichi, K., Hasegawa, T., Saito, K., Yamada, Y., Seishima, M., Sekikawa, K., Kim, H.C., Nabeshima, T., 2004. Role of tumor necrosis factor-alpha in methamphetamineinduced drug dependence and neurotoxicity. J Neurosci 24, 2212-2225.

Nakashima, K., Wiese, S., Yanagisawa, M., Arakawa, H., Kimura, N., Hisatsune, T., Yoshida, K., Kishimoto, T., Sendtner, M., Taga, T., 1999. Developmental requirement of gp130 signaling in neuronal survival and astrocyte differentiation. J Neurosci 19, 5429-5434.

Narita, M., Miyatake, M., Shibasaki, M., Shindo, K., Nakamura, A., Kuzumaki, N., Nagumo, Y., Suzuki, T., 2006. Direct evidence of astrocytic modulation in the development of rewarding effects induced by drugs of abuse. Neuropsychopharmacology 31, 2476-2488.

Narita, M., Miyatake, M., Shibasaki, M., Tsuda, M., Koizumi, S., Yajima, Y., Inoue, K., Suzuki, T., 2005. Long-lasting change in brain dynamics induced by methamphetamine: enhancement of protein kinase Cdependent astrocytic response and behavioral sensitization. J Neurochem 93, 1383-1392.

Narita, M., Suzuki, M., Kuzumaki, N., Miyatake, M., Suzuki, T., 2008. Implication of activated astrocytes in the development of drug dependence: differences between methamphetamine and morphine. Ann $\mathrm{N}$ Y Acad Sci 1141, 96-104.

Narita, N., Hashimoto, K., Tomitaka, S., Minabe, Y., 1996a. Interactions of selective serotonin reuptake inhibitors with subtypes of sigma receptors in rat brain. Eur J Pharmacol 307, 117-119.

Narita, N., Hashimoto, K., Tomitaka, S., Minabe, Y., 1996b. Interactions of selective serotonin reuptake inhibitors with subtypes of sigma receptors in rat brain. European Journal of Pharmacology 307, 117-

119.

Narita, N.H., K. Tomitaka, S. Minabe, Y., 1996. Interactions of selective serotonin reuptake inhibitors with subtypes of sigma receptors in rat brain. Eur J Pharmacology 307, 117-119.

Navarro, G., Moreno, E., Aymerich, M., Marcellino, D., McCormick, P.J., Mallol, J., Cortes, A., Casado, V., Canela, E.I., Ortiz, J., Fuxe, K., Lluis, C., Ferre, S., Franco, R., 2010. Direct involvement of sigma-1 receptors in the dopamine D1 receptor-mediated effects of cocaine. Proc Natl Acad Sci U S A 107, 18676-18681.

Nedergaard, M., Verkhratsky, A., 2012. Artifact versus reality--how astrocytes contribute to synaptic events. Glia 60, 1013-1023.

Nestler, E.J., Barrot, M., DiLeone, R.J., Eisch, A.J., Gold, S.J., Monteggia, L.M., 2002a. Neurobiology of depression. Neuron 34, 13-25.

Nestler, E.J., Gould, E., Manji, H., Buncan, M., Duman, R.S., Greshenfeld, H.K., Hen, R., Koester, S., Lederhendler, I., Meaney, M., Robbins, T., Winsky, L., Zalcman, S., 2002b. Preclinical models: status of basic research in depression. Biol Psychiatry 52, 503-528.

Nguyen, E.C., McCracken, K.A., Liu, Y., Pouw, B., Matsumoto, R.R., 2005. Involvement of sigma (sigma) receptors in the acute actions of methamphetamine: receptor binding and behavioral studies.

Neuropharmacology 49, 638-645.

Nierenberg, A.A., 2001. Do some antidepressants work faster than others? J Clin Psychiatry 62 Suppl 15, 22-25. 
Niino, D., Komohara, Y., Murayama, T., Aoki, R., Kimura, Y., Hashikawa, K., Kiyasu, J., Takeuchi, M., Suefuji, N., Sugita, Y., Takeya, M., Ohshima, K., 2010. Ratio of M2 macrophage expression is closely associated with poor prognosis for Angioimmunoblastic T-cell lymphoma (AITL). Pathol Int 60, 278-283. Nishimura, T., Ishima, T., Iyo, M., Hashimoto, K., 2008. Potentiation of nerve growth factor-induced neurite outgrowth by fluvoxamine: role of sigma-1 receptors, IP3 receptors and cellular signaling pathways. PLoS ONE 3, e2558.

Novakova, M., Ela, C., Barg, J., Vogel, Z., Hasin, Y., Eilam, Y., 1995. Inotropic action of sigma receptor ligands in isolated cardiac myocytes from adult rats. Eur J Pharmacol 286, 19-30.

O'Callaghan, J.P., Miller, D.B., 1994. Neurotoxicity profiles of substituted amphetamines in the C57BL/6J mouse. J Pharmacol Exp Ther 270, 741-751.

O'Callaghan, J.P., Sriram, K., 2004. Focused microwave irradiation of the brain preserves in vivo protein phosphorylation: comparison with other methods of sacrifice and analysis of multiple phosphoproteins. J Neurosci Methods 135, 159-168.

O'Callaghan, J.P., Sriram, K., Miller, D.B., 2008. Defining "neuroinflammation". Ann N Y Acad Sci 1139, 318-330.

Okuyama, S., Imagawa, Y., Tomisawa, K., 1996. Behavioral evidence for modulation by sigma ligands of (+)MK-801-induced hyperlocomotion in monoamine-depleted mice. Neuropharmacology 35, 467-474. Oliva, A.A., Jr., Kang, Y., Sanchez-Molano, J., Furones, C., Atkins, C.M., 2012. STAT3 signaling after traumatic brain injury. J Neurochem 120, 710-720.

Pan, Y.X., Mei, J., Xu, J., Wan, B.L., Zuckerman, A., Pasternak, G.W., 1998. Cloning and characterization of a mouse sigma1 receptor. J Neurochem 70, 2279-2285.

Pande, A., Geneve, J., Scherrer, B., Smith, F., Leadbetter, R., de Meynard, C., 1999. A placebo-controlled trial of igmesine in the treatment of major depression. European Neuropsychopharmacology 9, 138. Panenka, W.J., Procyshyn, R.M., Lecomte, T., Macewan, G.W., Flynn, S.W., Honer, W.G., Barr, A.M., 2012. Methamphetamine use: A comprehensive review of molecular, preclinical and clinical findings. Drug Alcohol Depend.

Paul, R., Schaaff, N., Padberg, F., Moller, H.J., Frodl, T., 2009. Comparison of racemic ketamine and Sketamine in treatment-resistant major depression: report of two cases. World J Biol Psychiatry 10, 241244.

Perry, V.H., Nicoll, J.A., Holmes, C., 2010. Microglia in neurodegenerative disease. Nat Rev Neurol 6, 193201.

Phan, V.L., Alonso, G., Sandillon, F., Privat, A., Maurice, T., 2000. Therapeutic potentials of sigma1 receptor ligands against cognitive deficits in aging. Soc Neurosci Abstr 26, 2172.

Pharmaprojects, 2004. Igmesine hydrochloride, accession number 15962. PJB Publications, Ltd. Policy, O.o.N.D.C., 2004. The economic costs of drug abuse in the United States, 1992-2002.

Washington, DC: Executive Office of the President Publication No: 207303.

Popik, P., Kos, T., Sowa-Kucma, M., Nowak, G., 2008. Lack of persistent effects of ketamine in rodent models of depression. Psychopharmacology (Berl) 198, 421-430.

Prezzavento, O., Campisi, A., Ronsisvalle, S., Li Volti, G., Marrazzo, A., Bramanti, V., Cannavo, G., Vanella, L., Cagnotto, A., Mennini, T., lentile, R., Ronsisvalle, G., 2007. Novel sigma receptor ligands: synthesis and biological profile. J Med Chem 50, 951-961.

Price, R.B., Nock, M.K., Charney, D.S., Mathew, S.J., 2009. Effects of intravenous ketamine on explicit and implicit measures of suicidality in treatment-resistant depression. Biol Psychiatry 66, 522-526.

Pu, C., Fisher, J.E., Cappon, G.D., Vorhees, C.V., 1994. The effects of amfonelic acid, a dopamine uptake inhibitor, on methamphetamine-induced dopaminergic terminal degeneration and astrocytic response in rat striatum. Brain Res 649, 217-224.

Quirion, R., Hammer, R.P., Jr., Herkenham, M., Pert, C.B., 1981. Phencyclidine (angel dust)/sigma "opiate" receptor: visualization by tritium-sensitive film. Proc Natl Acad Sci U S A 78, 5881-5885. 
Raivich, G., Bohatschek, M., Kloss, C.U., Werner, A., Jones, L.L., Kreutzberg, G.W., 1999. Neuroglial activation repertoire in the injured brain: graded response, molecular mechanisms and cues to physiological function. Brain Res Brain Res Rev 30, 77-105.

Rawson, R.A., Condon, T.P., 2007. Why do we need an Addiction supplement focused on methamphetamine? Addiction 102 Suppl 1, 1-4.

Rich, J.D., Wakeman, S.E., Dickman, S.L., 2011. Medicine and the epidemic of incarceration in the United States. N Engl J Med 364, 2081-2083.

Riddle, E.L., Fleckenstein, A.E., Hanson, G.R., 2006. Mechanisms of methamphetamine-induced dopaminergic neurotoxicity. AAPS J 8, E413-418.

Robson, M.J., Noorbakhsh, B., Seminerio, M.J., Matsumoto, R.R., 2012. Sigma-1 receptors: potential targets for the treatment of substance abuse. Curr Pharm Des 18, 902-919.

Robson, M.J., Turner, R.C., Naser, Z.J., McCurdy, C.R., Huber, J.D., Matsumoto, R.R., 2013. SN79, a sigma receptor ligand, blocks methamphetamine-induced microglial activation and cytokine upregulation. Exp Neurol.

Rodvelt, K.R., Lever, S.Z., Lever, J.R., Blount, L.R., Fan, K.H., Miller, D.K., 2011a. SA 4503 attenuates cocaine-induced hyperactivity and enhances methamphetamine substitution for a cocaine discriminative stimulus. Pharmacol Biochem Behav 97, 676-682.

Rodvelt, K.R., Miller, D.K., 2010. Could sigma receptor ligands be a treatment for methamphetamine addiction? Curr Drug Abuse Rev 3, 156-162.

Rodvelt, K.R., Oelrichs, C.E., Blount, L.R., Fan, K.H., Lever, S.Z., Lever, J.R., Miller, D.K., 2011b. The sigma receptor agonist SA4503 both attenuates and enhances the effects of methamphetamine. Drug Alcohol Depend.

Roehr, B., 2005. Half a million Americans use methamphetamine every week. BMJ 331, 476.

Rolan, P., Hutchinson, M., Johnson, K., 2009. Ibudilast: a review of its pharmacology, efficacy and safety in respiratory and neurological disease. Expert Opin Pharmacother 10, 2897-2904.

Romanelli, F., Smith, K.M., 2006. Clinical effects and management of methamphetamine abuse.

Pharmacotherapy 26, 1148-1156.

Ron, D., Walter, P., 2007. Signal integration in the endoplasmic reticulum unfolded protein response. Nat Rev Mol Cell Biol 8, 519-529.

Ruscher, K., Inacio, A.R., Valind, K., Rowshan Ravan, A., Kuric, E., Wieloch, T., 2012. Effects of the sigma-1 receptor agonist 1-(3,4-dimethoxyphenethyl)-4-(3-phenylpropyl)-piperazine dihydro-chloride on inflammation after stroke. PLoS One 7, e45118.

Ruscher, K., Shamloo, M., Rickhag, M., Ladunga, I., Soriano, L., Gisselsson, L., Toresson, H., Ruslim-Litrus, L., Oksenberg, D., Urfer, R., Johansson, B.B., Nikolich, K., Wieloch, T., 2011. The sigma-1 receptor enhances brain plasticity and functional recovery after experimental stroke. Brain 134, 732-746. Sabino, V., Cottone, P., Parylak, S.L., Steardo, L., Zorrilla, E.P., 2009. Sigma-1 receptor knockout mice display a depressive-like phenotype. Behav Brain Res 198, 472-476.

Saijo, K., Glass, C.K., 2011. Microglial cell origin and phenotypes in health and disease. Nat Rev Immunol 11, 775-787.

Schmidt, A., Lebel, L., Koe, B.K., Seeger, T., Heym, J., 1989. Sertraline potently displaces (+)-[3H]3-PPP binding to sigma sites in rat brain. Eur J Pharmacol 165, 335-336.

Schmidt, A.L., L. Koe, B. Seeger, T. Heym, J., 1989. Sertraline potently displaces (+)-[3H]3-PPP binding to sigma site in rat brain. Eur J Pharmacology 165, 335-336.

Schmitz, C., Hof, P.R., 2005. Design-based stereology in neuroscience. Neuroscience 130, 813-831. Schmued, L.C., Hopkins, K.J., 2000. Fluoro-Jade: novel fluorochromes for detecting toxicant-induced neuronal degeneration. Toxicol Pathol 28, 91-99.

Schwendt, M., Rocha, A., See, R.E., Pacchioni, A.M., McGinty, J.F., Kalivas, P.W., 2009. Extended methamphetamine self-administration in rats results in a selective reduction of dopamine transporter 
levels in the prefrontal cortex and dorsal striatum not accompanied by marked monoaminergic depletion. J Pharmacol Exp Ther 331, 555-562.

Seeman, P., Guan, H.C., Hirbec, H., 2009. Dopamine D2High receptors stimulated by phencyclidines, lysergic acid diethylamide, salvinorin A, and modafinil. Synapse 63, 698-704.

Sekine, Y., Iyo, M., Ouchi, Y., Matsunaga, T., Tsukada, H., Okada, H., Yoshikawa, E., Futatsubashi, M., Takei, N., Mori, N., 2001. Methamphetamine-related psychiatric symptoms and reduced brain dopamine transporters studied with PET. Am J Psychiatry 158, 1206-1214.

Sekine, Y., Minabe, Y., Ouchi, Y., Takei, N., Iyo, M., Nakamura, K., Suzuki, K., Tsukada, H., Okada, H., Yoshikawa, E., Futatsubashi, M., Mori, N., 2003. Association of dopamine transporter loss in the orbitofrontal and dorsolateral prefrontal cortices with methamphetamine-related psychiatric symptoms. Am J Psychiatry 160, 1699-1701.

Sekine, Y., Ouchi, Y., Sugihara, G., Takei, N., Yoshikawa, E., Nakamura, K., Iwata, Y., Tsuchiya, K.J., Suda, S., Suzuki, K., Kawai, M., Takebayashi, K., Yamamoto, S., Matsuzaki, H., Ueki, T., Mori, N., Gold, M.S., Cadet, J.L., 2008. Methamphetamine causes microglial activation in the brains of human abusers. J Neurosci 28, 5756-5761.

Sekine, Y., Ouchi, Y., Takei, N., Yoshikawa, E., Nakamura, K., Futatsubashi, M., Okada, H., Minabe, Y., Suzuki, K., Iwata, Y., Tsuchiya, K.J., Tsukada, H., Iyo, M., Mori, N., 2006. Brain serotonin transporter density and aggression in abstinent methamphetamine abusers. Arch Gen Psychiatry 63, 90-100. Seminerio, M.J., Hansen, R., Kaushal, N., Zhang, H.T., McCurdy, C.R., Matsumoto, R.R., 2012a. The evaluation of AZ66, an optimized sigma receptor antagonist, against methamphetamine-induced dopaminergic neurotoxicity and memory impairment in mice. Int J Neuropsychopharmacol, 1-12. Seminerio, M.J., Kaushal, N., Shaikh, J., Huber, J.D., Coop, A., Matsumoto, R.R., 2011. Sigma (sigma) receptor ligand, AC927 ( $\mathrm{N}$-phenethylpiperidine oxalate), attenuates methamphetamine-induced hyperthermia and serotonin damage in mice. Pharmacol Biochem Behav 98, 12-20.

Seminerio, M.J., Robson, M.J., Abdelazeem, A.H., Mesangeau, C., Jamalapuram, S., Avery, B.A., McCurdy, C.R., Matsumoto, R.R., 2012b. Synthesis and pharmacological characterization of a novel sigma receptor ligand with improved metabolic stability and antagonistic effects against methamphetamine. AAPS J 14, 43-51.

Seo, J., Fortuno, E.S., 3rd, Suh, J.M., Stenesen, D., Tang, W., Parks, E.J., Adams, C.M., Townes, T., Graff, J.M., 2009. Atf4 regulates obesity, glucose homeostasis, and energy expenditure. Diabetes 58, $2565-$

2573.

Sharkey, J., Glen, K.A., Wolfe, S., Kuhar, M.J., 1988. Cocaine binding at sigma receptors. Eur J Pharmacol 149, 171-174.

Shioda, N., Ishikawa, K., Tagashira, H., Ishizuka, T., Yawo, H., Fukunaga, K., 2012. Expression of a Truncated Form of the Endoplasmic Reticulum Chaperone Protein, sigma1 Receptor, Promotes Mitochondrial Energy Depletion and Apoptosis. J Biol Chem 287, 23318-23331.

Silva, R.M., Ries, V., Oo, T.F., Yarygina, O., Jackson-Lewis, V., Ryu, E.J., Lu, P.D., Marciniak, S.J., Ron, D., Przedborski, S., Kholodilov, N., Greene, L.A., Burke, R.E., 2005. CHOP/GADD153 is a mediator of apoptotic death in substantia nigra dopamine neurons in an in vivo neurotoxin model of parkinsonism. J Neurochem 95, 974-986.

Sircar, R., Nichtenhauser, R., leni, J.R., Zukin, S.R., 1986. Characterization and autoradiographic visualization of (+)-[3H]SKF10,047 binding in rat and mouse brain: further evidence for phencyclidine/"sigma opiate" receptor commonality. J Pharmacol Exp Ther 237, 681-688.

Siu, F., Bain, P.J., LeBlanc-Chaffin, R., Chen, H., Kilberg, M.S., 2002. ATF4 is a mediator of the nutrientsensing response pathway that activates the human asparagine synthetase gene. J Biol Chem 277, 24120-24127.

Skuza, G., 2003. Potential antidepressant activity of sigma ligands. Pol J Pharmacol 55, 923-934. 
Skuza, G., Rogoz, Z., 2002. A potential antidepressant activity of SA4503, a selective sigma 1 receptor agonist. Behav Pharmacol 13, 537-543.

Skuza, G., Rogoz, Z., 2003. Sigma1 receptor antagonists attenuate antidepressant-like effect induced by co-administration of 1,3 di-o-tolylguanidine (DTG) and memantine in the forced swimming test in rats. Pol J Pharmacol 55, 1149-1152.

Slattery, D.A., Hudson, A.L., Nutt, D.J., 2004. Invited review: the evolution of antidepressant mechanisms. Fundam Clin Pharmacol 18, 1-21.

Smith, D.W., Bailes, J.E., Fisher, J.A., Robles, J., Turner, R.C., Mills, J.D., 2012a. Internal jugular vein compression mitigates traumatic axonal injury in a rat model by reducing the intracranial slosh effect. Neurosurgery 70, 740-746.

Smith, J.A., Das, A., Ray, S.K., Banik, N.L., 2012b. Role of pro-inflammatory cytokines released from microglia in neurodegenerative diseases. Brain Res Bull 87, 10-20.

Son, J.S., Kwon, Y.B., 2010. Sigma-1 receptor antagonist BD1047 reduces allodynia and spinal ERK phosphorylation following chronic compression of dorsal root ganglion in rats. Korean J Physiol Pharmacol 14, 359-364.

Sonsalla, P.K., Nicklas, W.J., Heikkila, R.E., 1989. Role for excitatory amino acids in methamphetamineinduced nigrostriatal dopaminergic toxicity. Science 243, 398-400.

Spitzer, D., Simon, P.O., Jr., Kashiwagi, H., Xu, J., Zeng, C., Vangveravong, S., Zhou, D., Chang, K., McDunn, J.E., Hornick, J.R., Goedegebuure, P., Hotchkiss, R.S., Mach, R.H., Hawkins, W.G., 2012. Use of multifunctional sigma-2 receptor ligand conjugates to trigger cancer-selective cell death signaling.

Cancer Res 72, 201-209.

Sriram, K., Benkovic, S.A., Hebert, M.A., Miller, D.B., O'Callaghan, J.P., 2004. Induction of gp130-related cytokines and activation of JAK2/STAT3 pathway in astrocytes precedes up-regulation of glial fibrillary acidic protein in the 1-methyl-4-phenyl-1,2,3,6-tetrahydropyridine model of neurodegeneration: key signaling pathway for astrogliosis in vivo? J Biol Chem 279, 19936-19947.

Sriram, K., Miller, D.B., O'Callaghan, J.P., 2006. Minocycline attenuates microglial activation but fails to mitigate striatal dopaminergic neurotoxicity: role of tumor necrosis factor-alpha. J Neurochem 96, 706718.

Stadlin, A., Lau, J.W., Szeto, Y.K., 1998. A selective regional response of cultured astrocytes to methamphetamine. Ann N Y Acad Sci 844, 108-121.

Stahl, S.M., 2005. Antidepressant treatment of psychotic major depression: potential role of the sigma receptor. CNS Spectr 10, 319-323.

Stefanski, R., Justinova, Z., Hayashi, T., Takebayashi, M., Goldberg, S.R., Su, T.P., 2004. Sigma1 receptor upregulation after chronic methamphetamine self-administration in rats: a study with yoked controls. Psychopharmacology (Berl) 175, 68-75.

Stephans, S.E., Yamamoto, B.K., 1994. Methamphetamine-induced neurotoxicity: roles for glutamate and dopamine efflux. Synapse 17, 203-209.

Su, T.P., 1982. Evidence for sigma opioid receptor: binding of [3H]SKF-10047 to etorphine-inaccessible sites in guinea-pig brain. J Pharmacol Exp Ther 223, 284-290.

Su, T.P., Hayashi, T., 2003. Understanding the molecular mechanism of sigma-1 receptors: towards a hypothesis that sigma-1 receptors are intracellular amplifiers for signal transduction. Curr Med Chem 10, 2073-2080.

Su, T.P., Hayashi, T., Maurice, T., Buch, S., Ruoho, A.E., 2010a. The sigma-1 receptor chaperone as an inter-organelle signaling modulator. Trends Pharmacol Sci 31, 557-566.

Su, T.P., Hayashi, T., Maurice, T., Buch, S., Ruoho, A.E., 2010b. The sigma-1 receptor chaperone as an inter-organelle signaling modulator. Trends Pharmacol Sci. 
Sugimoto, Y., Tagawa, N., Kobayashi, Y., Mitsui-Saito, K., Hotta, Y., Yamada, J., 2012. Involvement of the sigma1 receptor in the antidepressant-like effects of fluvoxamine in the forced swimming test in comparison with the effects elicited by paroxetine. Eur J Pharmacol 696, 96-100.

Szegezdi, E., Logue, S.E., Gorman, A.M., Samali, A., 2006. Mediators of endoplasmic reticulum stressinduced apoptosis. EMBO Rep 7, 880-885.

Tabas, I., Ron, D., 2011. Integrating the mechanisms of apoptosis induced by endoplasmic reticulum stress. Nat Cell Biol 13, 184-190.

Takahashi, S., Miwa, T., Horikomi, K., 2000. Involvement of sigma 1 receptors in methamphetamineinduced behavioral sensitization in rats. Neurosci Lett 289, 21-24.

Takebayashi, M., Hayashi, T., Su, T.P., 2002. Nerve growth factor-induced neurite sprouting in PC12 cells involves sigma-1 receptors: implications for antidepressants. J Pharmacol Exp Ther 303, 1227-1237. Takebayashi, M., Hayashi, T., Su, T.P., 2004. A perspective on the new mechanism of antidepressants: neuritogenesis through sigma-1 receptors. Pharmacopsychiatry 37 Suppl 3, S208-213.

Takeichi, T., Wang, E.L., Kitamura, O., 2012. The effects of low-dose methamphetamine pretreatment on endoplasmic reticulum stress and methamphetamine neurotoxicity in the rat midbrain. Leg Med (Tokyo) 14, 69-77.

Tam, S.W., Cook, L., 1984. Sigma opiates and certain antipsychotic drugs mutually inhibit (+)-[3H] SKF 10,047 and $[3 \mathrm{H}]$ haloperidol binding in guinea pig brain membranes. Proc Natl Acad Sci U S A 81, 56185621.

Tan, F., Guio-Aguilar, P.L., Downes, C., Zhang, M., O'Donovan, L., Callaway, J.K., Crack, P.J., 2010. The sigma 1 receptor agonist 4-PPBP elicits ERK1/2 phosphorylation in primary neurons: a possible mechanism of neuroprotective action. Neuropharmacology 59, 416-424.

Tanibuchi, Y., Shimagami, M., Fukami, G., Sekine, Y., lyo, M., Hashimoto, K., 2010. A case of methamphetamine use disorder treated with the antibiotic drug minocycline. Gen Hosp Psychiatry 32, 559 e551-553.

Tata, D.A., Yamamoto, B.K., 2008. Chronic stress enhances methamphetamine-induced extracellular glutamate and excitotoxicity in the rat striatum. Synapse 62, 325-336.

Terleckyj, I., Sonsalla, P.K., 1994. The sigma receptor ligand (+/-)-BMY 14802 prevents methamphetamine-induced dopaminergic neurotoxicity via interactions at dopamine receptors. J Pharmacol Exp Ther 269, 44-50.

Thomas, D.M., Dowgiert, J., Geddes, T.J., Francescutti-Verbeem, D., Liu, X., Kuhn, D.M., 2004a. Microglial activation is a pharmacologically specific marker for the neurotoxic amphetamines. Neurosci Lett 367, 349-354.

Thomas, D.M., Francescutti-Verbeem, D.M., Liu, X., Kuhn, D.M., 2004b. Identification of differentially regulated transcripts in mouse striatum following methamphetamine treatment--an oligonucleotide microarray approach. J Neurochem 88, 380-393.

Thomas, D.M., Kuhn, D.M., 2005. Attenuated microglial activation mediates tolerance to the neurotoxic effects of methamphetamine. J Neurochem 92, 790-797.

Thomas, D.M., Walker, P.D., Benjamins, J.A., Geddes, T.J., Kuhn, D.M., 2004c. Methamphetamine neurotoxicity in dopamine nerve endings of the striatum is associated with microglial activation. $J$ Pharmacol Exp Ther 311, 1-7.

Todd, G., Noyes, C., Flavel, S.C., Della Vedova, C.B., Spyropoulos, P., Chatterton, B., Berg, D., White, J.M., 2013. Illicit stimulant use is associated with abnormal substantia nigra morphology in humans. PLoS One 8, e56438.

Tsai, S.Y., Hayashi, T., Mori, T., Su, T.P., 2009. Sigma-1 receptor chaperones and diseases. Cent Nerv Syst Agents Med Chem 9, 184-189.

Tsai, S.Y., Rothman, R.K., Su, T.P., 2012. Insights into the Sigma-1 receptor chaperone's cellular functions: a microarray report. Synapse 66, 42-51. 
Tsaytler, P., Harding, H.P., Ron, D., Bertolotti, A., 2011. Selective inhibition of a regulatory subunit of protein phosphatase 1 restores proteostasis. Science 332, 91-94.

Tsuji, T., Asanuma, M., Miyazaki, I., Miyoshi, K., Ogawa, N., 2009. Reduction of nuclear peroxisome proliferator-activated receptor gamma expression in methamphetamine-induced neurotoxicity and neuroprotective effects of ibuprofen. Neurochem Res 34, 764-774.

Tu, Z., Xu, J., Jones, L.A., Li, S., Zeng, D., Kung, M.P., Kung, H.F., Mach, R.H., 2010. Radiosynthesis and biological evaluation of a promising sigma(2)-receptor ligand radiolabeled with fluorine-18 or iodine-125 as a PET/SPECT probe for imaging breast cancer. Appl Radiat Isot 68, 2268-2273.

Tuerxun, T., Numakawa, T., Adachi, N., Kumamaru, E., Kitazawa, H., Kudo, M., Kunugi, H., 2010. SA4503, a sigma-1 receptor agonist, prevents cultured cortical neurons from oxidative stress-induced cell death via suppression of MAPK pathway activation and glutamate receptor expression. Neurosci Lett 469, $303-$ 308.

Turner, R.C., Naser, Z.J., Bailes, J.E., Smith, D.W., Fisher, J.A., Rosen, C.L., 2012. Effect of slosh mitigation on histologic markers of traumatic brain injury: laboratory investigation. J Neurosurg 117, 1110-1118.

Ujike, H., Kanzaki, A., Okumura, K., Akiyama, K., Otsuki, S., 1992a. Sigma antagonist BMY 14802 prevents methamphetamine-induced sensitization. Life Sci 50, PL129-134.

Ujike, H., Kuroda, S., Otsuki, S., 1996. Sigma receptor antagonists block the development of sensitization to cocaine. Eur J Pharmacol 296, 123-128.

Ujike, H., Okumura, K., Zushi, Y., Akiyama, K., Otsuki, S., 1992b. Persistent supersensitivity of sigma receptors develops during repeated methamphetamine treatment. Eur J Pharmacol 211, 323-328. Ukai, M., Maeda, H., Nanya, Y., Kameyama, T., Matsuno, K., 1998. Beneficial effects of acute and repeated administrations of sigma receptor agonists on behavioral despair in mice exposed to tail suspension. Pharmacol Biochem Behav 61, 247-252.

Ullian, E.M., Christopherson, K.S., Barres, B.A., 2004. Role for glia in synaptogenesis. Glia 47, 209-216. Van Wagoner, N.J., Benveniste, E.N., 1999. Interleukin-6 expression and regulation in astrocytes. J Neuroimmunol 100, 124-139.

Van Wagoner, N.J., Choi, C., Repovic, P., Benveniste, E.N., 2000. Oncostatin M regulation of interleukin-6 expression in astrocytes: biphasic regulation involving the mitogen-activated protein kinases ERK1/2 and p38. J Neurochem 75, 563-575.

Vaupel, D.B., 1983. Naltrexone fails to antagonize the sigma effects of PCP and SKF 10,047 in the dog. Eur J Pharmacol 92, 269-274.

Villard, V.M.J.C., N. Maurice, T., 2011. Pharmacological interaction with the sigma 1-receptor in the acute behavioral effects of antidepressants. Journal of Pharmacological Sciences 115, 279-292.

Vilner, B.J., Bowen, W.D., 1993. Sigma receptor-active neuroleptics are cytotoxic to C6 glioma cells in culture. Eur J Pharmacol 244, 199-201.

Vilner, B.J., Bowen, W.D., 2000. Modulation of cellular calcium by sigma-2 receptors: release from intracellular stores in human SK-N-SH neuroblastoma cells. J Pharmacol Exp Ther 292, 900-911. Vilner, B.J., de Costa, B.R., Bowen, W.D., 1995. Cytotoxic effects of sigma ligands: sigma receptormediated alterations in cellular morphology and viability. J Neurosci 15, 117-134.

Volkow, N.D., Chang, L., Wang, G.J., Fowler, J.S., Franceschi, D., Sedler, M., Gatley, S.J., Miller, E., Hitzemann, R., Ding, Y.S., Logan, J., 2001a. Loss of dopamine transporters in methamphetamine abusers recovers with protracted abstinence. J Neurosci 21, 9414-9418.

Volkow, N.D., Chang, L., Wang, G.J., Fowler, J.S., Leonido-Yee, M., Franceschi, D., Sedler, M.J., Gatley, S.J., Hitzemann, R., Ding, Y.S., Logan, J., Wong, C., Miller, E.N., 2001b. Association of dopamine transporter reduction with psychomotor impairment in methamphetamine abusers. Am J Psychiatry 158, 377-382.

Volz, H.P., Stoll, K.D., 2004. Clinical trials with sigma ligands. Pharmacopsychiatry 37 Suppl 3, S214-220. 
Walter, P., Ron, D., 2011. The unfolded protein response: from stress pathway to homeostatic regulation. Science 334, 1081-1086.

Wang, C., Huang, Z., Du, Y., Cheng, Y., Chen, S., Guo, F., 2010. ATF4 regulates lipid metabolism and thermogenesis. Cell Res 20, 174-184.

Wang, D., Noda, Y., Tsunekawa, H., Zhou, Y., Miyazaki, M., Senzaki, K., Nitta, A., Nabeshima, T., 2007 a.

Role of N-methyl-D-aspartate receptors in antidepressant-like effects of sigma 1 receptor agonist 1-(3,4-

dimethoxyphenethyl)-4-(3-phenylpropyl)piperazine dihydrochloride (SA-4503) in olfactory

bulbectomized rats. J Pharmacol Exp Ther 322, 1305-1314.

Wang, G.J., Smith, L., Volkow, N.D., Telang, F., Logan, J., Tomasi, D., Wong, C.T., Hoffman, W., Jayne, M., Alia-Klein, N., Thanos, P., Fowler, J.S., 2012. Decreased dopamine activity predicts relapse in methamphetamine abusers. Mol Psychiatry 17, 918-925.

Wang, G.J., Volkow, N.D., Chang, L., Miller, E., Sedler, M., Hitzemann, R., Zhu, W., Logan, J., Ma, Y., Fowler, J.S., 2004. Partial recovery of brain metabolism in methamphetamine abusers after protracted abstinence. Am J Psychiatry 161, 242-248.

Wang, J., Mack, A.L., Coop, A., Matsumoto, R.R., 2007b. Novel sigma (sigma) receptor agonists produce antidepressant-like effects in mice. Eur Neuropsychopharmacol 17, 708-716.

Weatherspoon, J.K., Gonzalez-Alvear, G.M., Frank, A.R., Werling, L.L., 1996. Regulation of [3H] dopamine release from mesolimbic and mesocortical areas of guinea pig brain by sigma receptors. Schizophr Res 21, 51-62.

Weber, E., Sonders, M., Quarum, M., McLean, S., Pou, S., Keana, J.F., 1986. 1,3-Di(2-[5-

$3 \mathrm{H}$ ]tolyl)guanidine: a selective ligand that labels sigma-type receptors for psychotomimetic opiates and antipsychotic drugs. Proc Natl Acad Sci U S A 83, 8784-8788.

Weiser, S.D., Patrick, S.L., Mascarella, S.W., Downing-Park, J., Bai, X., Carroll, F.I., Walker, J.M., Patrick, R.L., 1995. Stimulation of rat striatal tyrosine hydroxylase activity following intranigral administration of sigma receptor ligands. Eur J Pharmacol 275, 1-7.

Wheeler, K.T., Wang, L.M., Wallen, C.A., Childers, S.R., Cline, J.M., Keng, P.C., Mach, R.H., 2000. Sigma-2 receptors as a biomarker of proliferation in solid tumours. Br J Cancer 82, 1223-1232.

Wolfe, S.A., Jr., Ha, B.K., Whitlock, B.B., Saini, P., 1997. Differential localization of three distinct binding sites for sigma receptor ligands in rat spleen. J Neuroimmunol 72, 45-58.

Woods, S.P., Rippeth, J.D., Conover, E., Gongvatana, A., Gonzalez, R., Carey, C.L., Cherner, M., Heaton, R.K., Grant, I., 2005. Deficient strategic control of verbal encoding and retrieval in individuals with methamphetamine dependence. Neuropsychology 19, 35-43.

$\mathrm{Xu}, \mathrm{X}$., Domino, E.F., 1997. Cross-sensitization between phencyclidine and (-) but not (+) pentazocine. Pharmacol Biochem Behav 56, 205-210.

Xu, Y.T., Kaushal, N., Shaikh, J., Wilson, L.L., Mesangeau, C., McCurdy, C.R., Matsumoto, R.R., 2010. A novel substituted piperazine, CM156, attenuates the stimulant and toxic effects of cocaine in mice. J Pharmacol Exp Ther 333, 491-500.

Yan, H.C., Cao, X., Das, M., Zhu, X.H., Gao, T.M., 2010. Behavioral animal models of depression. Neurosci Bull 26, 327-337.

Yang, S., Alkayed, N.J., Hurn, P.D., Kirsch, J.R., 2009. Cyclic adenosine monophosphate response element-binding protein phosphorylation and neuroprotection by 4-phenyl-1-(4-phenylbutyl) piperidine (PPBP). Anesth Analg 108, 964-970.

Yao, H., Yang, Y., Kim, K.J., Bethel-Brown, C., Gong, N., Funa, K., Gendelman, H.E., Su, T.P., Wang, J.Q., Buch, S., 2010. Molecular mechanisms involving sigma receptor-mediated induction of MCP-1: implication for increased monocyte transmigration. Blood 115, 4951-4962.

Yilmaz, A., Schulz, D., Aksoy, A., Canbeyli, R., 2002. Prolonged effect of an anesthetic dose of ketamine on behavioral despair. Pharmacol Biochem Behav 71, 341-344. 
Young, G.A., Khazan, N., 1984. Differential neuropharmacological effects of mu, kappa and sigma opioid agonists on cortical EEG power spectra in the rat. Stereospecificity and naloxone antagonism. Neuropharmacology 23, 1161-1165.

Yue, X., Qiao, D., Wang, A., Tan, X., Li, Y., Liu, C., Wang, H., 2012. CD200 attenuates methamphetamineinduced microglial activation and dopamine depletion. J Huazhong Univ Sci Technolog Med Sci 32, 415421.

Zamanian, J.L., Xu, L., Foo, L.C., Nouri, N., Zhou, L., Giffard, R.G., Barres, B.A., 2012. Genomic analysis of reactive astrogliosis. J Neurosci 32, 6391-6410.

Zarate, C.A., Jr., Singh, J.B., Carlson, P.J., Brutsche, N.E., Ameli, R., Luckenbaugh, D.A., Charney, D.S., Manji, H.K., 2006. A randomized trial of an N-methyl-D-aspartate antagonist in treatment-resistant major depression. Arch Gen Psychiatry 63, 856-864.

Zeng, C., Rothfuss, J., Zhang, J., Chu, W., Vangveravong, S., Tu, Z., Pan, F., Chang, K.C., Hotchkiss, R., Mach, R.H., 2012. Sigma-2 ligands induce tumour cell death by multiple signalling pathways. Br J Cancer 106, 693-701.

Zeng, C., Vangveravong, S., Xu, J., Chang, K.C., Hotchkiss, R.S., Wheeler, K.T., Shen, D., Zhuang, Z.P., Kung, H.F., Mach, R.H., 2007. Subcellular localization of sigma-2 receptors in breast cancer cells using two-photon and confocal microscopy. Cancer Res 67, 6708-6716.

Zhang, L., Kitaichi, K., Fujimoto, Y., Nakayama, H., Shimizu, E., Iyo, M., Hashimoto, K., 2006. Protective effects of minocycline on behavioral changes and neurotoxicity in mice after administration of methamphetamine. Prog Neuropsychopharmacol Biol Psychiatry 30, 1381-1393.

Zhang, Z., Zhang, Z.Y., Wu, Y., Schluesener, H.J., 2012. Lesional accumulation of CD163(+) macrophages/microglia in rat traumatic brain injury. Brain Res 1461, 102-110.

Zhong, Z., Wen, Z., Darnell, J.E., Jr., 1994. Stat3: a STAT family member activated by tyrosine phosphorylation in response to epidermal growth factor and interleukin-6. Science 264, 95-98. Zinszner, H., Kuroda, M., Wang, X., Batchvarova, N., Lightfoot, R.T., Remotti, H., Stevens, J.L., Ron, D., 1998. CHOP is implicated in programmed cell death in response to impaired function of the endoplasmic reticulum. Genes Dev 12, 982-995. 


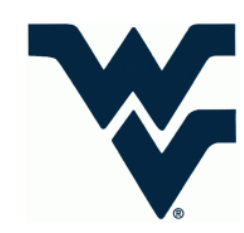

\section{MATTHEW J. ROBSON \\ 3524 Collins Ferry Rd \\ Morgantown, WV 26505}

(315) 246-8879

mjrobson@hsc.wvu.edu

\section{$\underline{\text { EDUCATION }}$}

West Virginia University

2008 - Present

Ph.D. Candidate - Pharmaceutical and Pharmacological Sciences

Expected Graduation Date - Summer 2013

Accepted Postdoctoral Position - Randy Blakely, Ph.D. Vanderbilt University

Canisius College

$2003-2007$

Bachelor of Science - Biochemistry

\section{WORK EXPERIENCE}

West Virginia University

2008 - Present

Graduate Student

Sub-Investigator Glaxo-Smith Kline Clinical Trial - XPXXXXX

2008- 2009

Dent Neurologic Institute Study Site- Buffalo, NY

Dent Neurologic Institute - Buffalo, NY

$2005-2008$

Research Associate

Eckerd's Pharmacy - Waterloo, NY

2005

Drug Associate

\section{ORGANIZATIONS/LEADERSHIP ROLES}

Graduate Student Organization - Vice President

2012- Present

West Virginia University

Drug Discovery Seminar Series Coordinator

2011-Present

West Virginia University School of Pharmacy

Graduate Student Organization - Member

2011-Present

West Virginia University

Student Representative - Laboratory Manager Search Committee

$2011-2012$

West Virginia University

Chair - American Association of Pharmaceutical Scientists

$2011-2012$

West Virginia University Student Chapter

Undergraduate Honors Thesis Committee Member

2010-2011

Colleen Beatty - West Virginia University 
Canisius College Chapter - Member

\section{PEER REVIEWED PUBLICATIONS}

Gengo FM, Rubin L, Robson M, Gengo MF, Mager DE, Rainka MR, Bates VE. Effects of ibuprofen on the magnitude and duration of aspirin's inhibition of platelet aggregation: clinical consequences in stroke prophylaxis. Journal of Clinical Pharmacology. 2008. 48(1): 117-122. PubMed ID: 18094224

Gengo FM, Rainka MR, Robson M, Gengo MF, Forrest A, Hourihane M, Bates VE. Prevalence of platelet nonresponsiveness to aspirin in patients treated for secondary stroke prophylaxis and in patients with recurrent ischemic events. Journal of Clinical Pharmacology. 2008. 48(3): 335-343. PubMed ID: 18223144

Fishback JA, Robson MJ, Xu Y, Matsumoto RR. Sigma receptors: potential targets for a new class of antidepressant drug. Pharmacology and Therapeutics. 2010. 127(3): 271-282. PubMed ID: 20438757

Kaushal N, Robson MJ, Vinnakota H, Narayanan S, Avery BA, McCurdy CR, Matsumoto RR. Synthesis and pharmacological evaluation of 6-acetyl-3-(4-(4-(4-fluorophenyl)piperazin-1yl)butyl)benzo[d]oxazol-2(3H)-one (SN79), a cocaine antagonist, in rodents. AAPS Journal. 2011. 13(3): 336-346. PubMed ID: 21494909

Mesangeau C, Amata E, Alsharif W, Seminerio MJ, Robson MJ, Matsumoto RR, Poupaert JH, McCurdy CR. Synthesis and pharmacological evaluation of indole-based sigma receptor ligands. European Journal of Medicinal Chemistry. 2011. 46(10): 5154-5161. PubMed ID: 21899931

Robson MJ, Elliott M, Seminerio MJ, Matsumoto RR. Evaluation of sigma ( $\sigma$ ) receptors in the antidepressant-like effects of ketamine in vitro and in vivo. European

Neuropsychopharmacology. 2012. 22(4): 308-317. PubMed ID: 21911285

Kaushal N, Elliott M, Robson MJ, Iyer AKV, Rojanasakul Y, Coop A, Matsumoto RR. AC927, a sigma ligand, blocks methamphetamine-induced release of dopamine and generation of reactive oxygen species in NG108-15 cells. Molecular Pharmacology. 2012. 81(3): 299-308. PubMed ID: 22101517 
Seminerio MJ, Robson MJ, Abdelazeem AH, Mesangeau C, Jamalapuram S, Avery BA, McCurdy CR, Matsumoto RR. Synthesis and pharmacological characterization of a novel sigma receptor ligand with improved metabolic stability and antagonistic effects against methamphetamine. AAPS Journal. 2012. 14(1): 43-51. PubMed ID: 22183188

Xu YT, Robson MJ, Szeszel-Fedorowicz W, Patel D, Rooney R, McCurdy CR, Matsumoto RR. CM156, a sigma receptor ligand, reverses cocaine-induced place conditioning and transcriptional responses in the brain. Pharmacology Biochemistry \& Behavior. 2012. 101(1): 174-180.

PubMed ID: 22234290

Robson MJ, Noorbakhsh B, Seminerio MJ, Matsumoto RR. Sigma-1 receptors: potential targets for the treatment of substance abuse. Current Pharmaceutical Design. 2012. 18: 902-919.

PubMed ID: 22288407

Seminerio MJ, Robson MJ, McCurdy CR, Matsumoto RR. Sigma receptor ligands attenuate methamphetamine-induced hyperthermia by a mechanism independent of IL-1 $\beta$ mRNA expression in the hypothalamus. European Journal of Pharmacology. 2012. 691(1-3): 103109. PubMed ID: 22820108

Kaushal N, Seminerio MJ, Robson MJ, McCurdy CR, Matsumoto RR. Pharmacological evaluation of SN79, a sigma receptor ligand against methamphetamine-induced neurotoxicity in vivo. European Neuropsychopharmacology. 2012. Accepted. PubMed ID: 22921523

Turner RC, Lucke-Wold B, Robson MJ, Bales JE. Repetitive traumatic brain injury and development of chronic traumatic encephalopathy: a potential role for biomarkers in diagnosis, prognosis and treatment? Frontiers in Neurotrauma. 2012. 3:186. PubMed ID: 23335911

Robson MJ, Seminerio MJ, McCurdy CR, Coop A, Matsumoto RR. Sigma receptor antagonist attenuation of methamphetamine-induced neurotoxicity is correlated to body temperature modulation. Pharmacological Reports. 2013. 65(2): 343-349.

Matsumoto, RR, Seminerio MJ, Turner RC, Robson MJ, O’Callaghan JP, Miller DB. Methamphetamine-induced hyperthermia: a comprehensive review. Pharmacology and Therapeutics. 2013. Submitted.

Robson MJ, McCurdy CR, Matsumoto RR. Methamphetamine causes PERK-mediated endoplasmic reticulum stress in NG108-15 cells through a mechanism independent of sigma receptors. European Neuropsychopharmacology. 2013. Submitted.

Robson MJ, Turner RC, Naser ZJ, McCurdy CR, Huber JD, Matsumoto RR. SN79, a sigma receptor ligand, blocks methamphetamine-induced microglial activation and cytokine upregulation. Experimental Neurology. 2013. Accepted. 
Turner RC, Naser ZJ, Logsdon AF, DiPasquale K, Jackson GJ, Robson MJ, Gettens RTT, Matsumoto RR, Rosen CL, Huber JD. Modeling clinically relevant blast parameters based on scaling principles produces functional and histological deficits in rats. Experimental Neurology. 2013. Submitted.

Robson MJ, Turner RC, Naser ZJ, Huber JD, McCurdy CR, Matsumoto RR. SN79, a sigma receptor ligand, attenuates methamphetamine-induced astrogliosis through a blockade of OSMR/gp130 signaling and STAT3 phosphorylation. Glia. 2013. In Preparation.

\section{PUBLISHED ABSTRACTS}

Gengo FM, Rainka M, Rubin L, Gengo M, Robson M, Bates V. Effects on the magnitude and time course of aspirin-induced inhibition of platelet aggregation by ibuprofen and the clinical consequences in secondary stroke prophylaxis. Journal of Clinical Pharmacology. 2006. 46(9): 1073.

Gengo FM, Robson M, Gengo MF, Rainka MR, Hourihane M, Bates VE. The prevalence of platelet non-responsiveness to aspirin in patients treated for secondary stroke prophylaxis and in patients with recurrent ischemic events. Stroke. 2006. 37(2): 715.

Gengo FM, Liana D, Robson M, Gengo M, Rainka M, Bates V. Effect of ascorbic acid on platelet responsiveness to clopidogrel in patients treated for secondary stroke prophylaxis. Stroke. 2008. 39: 624.

Gengo FM, Rainka M, Westphal E, Robson M, Gengo M, Hourihane M, Bates V. Platelet response to increased aspirin dose in patients whose platelets were non-responsive to lower aspirin doses. Stroke. 2009. 40(4): 22-23.

Gengo FM, Iyer V, Robson M, Gengo M, Rainka M, Forrest A. Effects of naproxen on the time course of aspirin-induced inhibition of platelet aggregation. Clinical Pharmacology and Therapeutics. 2009. 85(Suppl. 1): S40.

Robson MJ, Shaikh J, Elliott M, Xu YT, Healy JR, Matsumoto RR. The antidepressant-like actions of ketamine in vivo and in vitro: role of sigma receptors. Journal of Clinical Pharmacology. 2010. 50(9): 1084.

Gengo FM, Rainka M, Westphal E, Radecki L, Robson M, Hourihane M, Bates V. An assay to assess antiplatelet effects of dipyridamole at therapeutic concentrations. Journal of Clinical Pharmacology. 2010. 50(9): 1069.

Gengo FM, Robson M, Rainka M, Westphal E, Gengo M, Hourihane M, Bates V. The prevalence of platelet non-responsiveness to clopidogrel and the effect of cytochrome P450 1A2 inducing medications. Journal of Clinical Pharmacology. 2010. 50(9): 1069. 
Robson MJ, Chen D, Szeszel-Fedorowicz W, Matsumoto RR. Sigma receptor antagonists attenuate methamphetamine-induced genetic alterations in the striatum. Program No. 896.03. 2011 Neuroscience Meeting Planner. Washington, DC: Society for Neuroscience, 2011. Online.

Wollenberg L, Iyer V, Robson M, Gengo M, Gengo FM, Rainka M, Forrest A, Mager D. Application of a mechanism-based, population pharmacodynamic model of the time-course of platelet aggregation when naproxen and aspirin are administered alone and in combination. Pharmacotherapy. 2012. 32(5): e115.

Robson MJ, McCurdy CR, Matsumoto RR. Methamphetamine elicits PERK-mediated endoplasmic reticulum stress transcriptional responses in NG108-15 cells. Program No. 360.12. 2012 Neuroscience Meeting Planner. New Orleans, LA: Society for Neuroscience, 2012. Online.

Robson MJ, Turner RC, Naser ZJ, Huber JD, McCurdy CR, Matsumoto RR. SN79, a sigma receptor ligand, mitigates methamphetamine-induced astrocyte and microglial activation. FASEB J. 2013. 27(Meeting Abstract Supplement): 1175.2

Matsumoto RR, Kaushal N, Robson MJ, McCurdy CR, Coop AC. Attenuation of methamphetamine-induced neurotoxicity by selective sigma receptor ligands: in vivo and in vitro studies. FASEB J. 2013. 27(Meeting Abstract Supplement): 659.13

\section{POSTER PRESENTATIONS}

The Rapid-Acting Antidepressant Actions of Ketamine:

A Sigma Receptor Mediated Effect?

West Virginia University Neuroscience Retreat 2009. Morgantown, WV.

Robson MJ, Shaikh J, Xu Y, Healy J, Matsumoto RR.

The Antidepressant Actions of Ketamine:

A Study to Determine the Role of Sigma Receptors and

Time Course of These Effects in a Rodent Model.

West Virginia University Van Liere Research Day. Morgantown, WV.

Robson MJ, Shaikh J, Xu Y, Healy J, Matsumoto RR.

The Antidepressant-like Actions of Ketamine In Vivo and In Vitro: Role of 2010 Sigma Receptors.

$39^{\text {th }}$ Annual Meeting of the American College of Clinical Pharmacology. Baltimore, MD.

Student/Trainee Travel Award Received

Robson MJ, Shaikh J, Elliott M, Xu YT, Healy JR, Matsumoto RR. 
Wide Approach

West Virginia University Van Liere Research Day. Morgantown, WV

Robson MJ, Chen D, Szeszel-Fedorowicz W, Matsumoto RR.

AC927 Attenuates Methamphetamine-Induced Gene Changes: A Genome

Wide Approach

$3^{\text {rd }}$ Annual Regional AAPS Symposium- Duquesne University. Pittsburgh, PA.

$1^{\text {st }}$ Place Student Poster

Robson MJ, Chen D, Szeszel-Fedorowicz W, Matsumoto RR.

Sigma Receptor Antagonists Attenuate Methamphetamine-Induced Genetic

Alterations in the Striatum

Society for Neuroscience 2011- Washington, D.C.

Robson MJ, Chen D, Szeszel-Fedorowicz W, Matsumoto RR.

Methamphetamine-Induced Endoplasmic Reticulum Stress Involves the

Induction of PERK-Mediated Transcriptional Responses

West Virginia University Van Liere Research Day. Morgantown, WV

$2^{\text {nd }}$ Place Student Poster

Robson MJ, Kaushal N, Matsumoto RR.

Attenuation of Methamphetamine-Induced Neurotoxicity and Genetic

Alterations in the Striatum by Targeting Sigma Receptors

Center for Clinical and Translational Science Conference

University of Kentucky. Lexington, KY.

Robson MJ, Kaushal N, Matsumoto RR.

Methamphetamine Elicits PERK-mediated Endoplasmic Reticulum

Stress Transcriptional Responses in NG108-15 Cells

Society for Neuroscience 2012- New Orleans, LA

Robson MJ, McCurdy CR, Matsumoto RR.

SN79, A Sigma Receptor Antagonist, Mitigates Methamphetamine-Induced

Reactive Gliosis and Cytokine Upregulation

West Virginia University Van Liere Research Day. Morgantown, WV

$1^{\text {st }}$ Place Student Poster

Robson MJ, Turner RC, Naser ZJ, McCurdy CR, Matsumoto RR.

SN79, A Sigma Receptor Ligand, Mitigates Methamphetamine-Induced

Robson MJ, Turner RC, Naser ZJ, McCurdy CR, Matsumoto RR.

\section{INVITED PRESENTATIONS}


Sigma Receptors: Potential Targets for the Treatment of Psychostimulant Abuse 2011 Chestnut Ridge Drug Abuse Grand Rounds, November $1^{\text {st }}$

\section{SCIENTIFIC SOCIETY MEMBERSHIPS}

Phi Lambda Sigma Pharmacy Leadership Society - Alpha Eta Chapter

2013-Present

Rho Chi Pharmacy Honor Society - Alpha Mu Chapter

2012-Present

Society for Neuroscience (SfN)

American Society for Pharmacology and Experimental Therapeutics (ASPET)

International Drug Abuse Research Society (IDARS)

American Association of Pharmaceutical Scientists (AAPS)

2011-Present

2010-Present

2010-Present

2009-Present

\section{AWARDS/FELLOWSHIPS}

Abstract Selected for Best Abstract Competition

2013

ASPET - Neuropharmacology Section - Experimental Biology 2013

Graduate Student Research Award

2013

West Virginia University- School of Pharmacy

ASPET Washington Fellows Fellowship Recipient

2012-Present

American Society for Pharmacology and Experimental Therapeutics

Outstanding Achievement Award

2012

West Virginia University - School of Pharmacy

$3^{\text {rd }}$ Annual AAPS Regional Symposium

2011

$1^{\text {st }}$ Place- Poster Competition

STaR Symposium Student Abstract (1 of 10 selected from 560 submissions) 2010

Student/Trainee Award - American College of Clinical Pharmacology 2010

$39^{\text {th }}$ Annual Meeting - Baltimore, MD

Valuable Contributions to the School of Pharmacy

2009-2010

West Virginia University

BBS Training Scholarship - West Virginia University

2009-2010

Undergraduate Award for Excellence in Research

2006

SUNY- University at Buffalo

\section{VOLUNTEER/COMMUNITY SERVICE ACTIVITIES}

Intensive Outpatient Program

Chestnut Ridge Psychiatric Center, Morgantown, WV

Alternative Spring Break Leader (Canisius College)

Habitat for Humanity Volunteer Trip to Appalachia

Women's and Children's Hospital of Buffalo Volunteer

Alternative Spring Break Volunteer (Canisius College)

2006

Habitat for Humanity Volunteer Trip to Appalachia 
